\title{
Optimal experimental designs for functional magnetic resonance imaging
}

Citation for published version (APA):

Maus, B. (2011). Optimal experimental designs for functional magnetic resonance imaging. [Doctoral Thesis, Maastricht University]. Datawyse / Universitaire Pers Maastricht.

https://doi.org/10.26481/dis.20110420bm

Document status and date:

Published: 01/01/2011

DOI:

10.26481/dis.20110420bm

Document Version:

Publisher's PDF, also known as Version of record

\section{Please check the document version of this publication:}

- A submitted manuscript is the version of the article upon submission and before peer-review. There can be important differences between the submitted version and the official published version of record.

People interested in the research are advised to contact the author for the final version of the publication, or visit the DOI to the publisher's website.

- The final author version and the galley proof are versions of the publication after peer review.

- The final published version features the final layout of the paper including the volume, issue and page numbers.

Link to publication

\footnotetext{
General rights rights.

- You may freely distribute the URL identifying the publication in the public portal. please follow below link for the End User Agreement:

www.umlib.nl/taverne-license

Take down policy

If you believe that this document breaches copyright please contact us at:

repository@maastrichtuniversity.nl

providing details and we will investigate your claim.
}

Copyright and moral rights for the publications made accessible in the public portal are retained by the authors and/or other copyright owners and it is a condition of accessing publications that users recognise and abide by the legal requirements associated with these

- Users may download and print one copy of any publication from the public portal for the purpose of private study or research.

- You may not further distribute the material or use it for any profit-making activity or commercial gain

If the publication is distributed under the terms of Article $25 \mathrm{fa}$ of the Dutch Copyright Act, indicated by the "Taverne" license above, 
Optimal experimental designs for functional magnetic resonance imaging

Bärbel Maus 
ISBN: 9789461590527

Printing: Datawyse/Universitaire Pers Maastricht

(C) Copyright Bärbel Maus, Maastricht 2011

This dissertation was supported by the Netherlands Organisation for Scientific Research (NWO, grant number: 400-05-063), the School for Public Health and Primary Care (CAPHRI), Maastricht University, and the Interuniversity Graduate School of Psychometrics and Sociometrics (IOPS). 


\title{
Optimal experimental designs for functional magnetic resonance imaging
}

\author{
PROEFSCHRIFT
}

ter verkrijging van de graad van doctor aan de Universiteit Maastricht,

op gezag van de Rector Magnificus, Prof. mr. G.P.M.F. Mols, volgens het besluit van het College van Decanen,

in het openbaar te verdedigen

op woensdag 20 april 2011 om 16.00 uur

door

Bärbel Maus

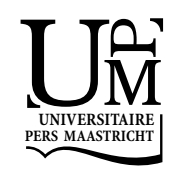


Promotoren

Prof. dr. M.P.F. Berger

Prof. dr. R. Goebel

\section{Copromotor}

Dr. G.J.P. van Breukelen

\section{Beoordelingscommissie}

Prof. dr. L.M.G. Curfs (voorzitter)

Dr. E. Formisano

Prof. dr. D.G. Norris (Radboud University Nijmegen)

Prof. dr. J. Stufken (University of Georgia, USA)

Dr. E.S. Tan 


\section{Contents}

1 Introduction $\quad 1$

1.1 Background to functional magnetic resonance imaging . . . . . . . . . . . 2

1.2 Design of fMRI experiments . . . . . . . . . . . . . . . . . . . . . . . . . . .

1.2.1 Blocked designs . . . . . . . . . . . . . . . . . . 7

1.2.2 Event-related designs . . . . . . . . . . . . . . . . . . . . 7

1.3 Models . . . . . . . . . . . . . . . . . . . . 9 9

1.3.1 Model 1: Linear estimation of hemodynamic response function . . . 9

1.3.2 Model 2: Detection . . . . . . . . . . . . . . . . . 11

1.3.3 Model 3: Nonlinear estimation of hemodynamic response function . 12

1.3.4 Model 4: Mixed-effects model for detection . . . . . . . . . . . . . 13

1.3.5 Error structure . . . . . . . . . . . . . . . . . . . 14

1.4 Optimal design . . . . . . . . . . . . . . . . . . . . . 14

1.4.1 A-optimality criterion . . . . . . . . . . . . 15

$1.4 .2 \quad D$-optimality criterion . . . . . . . . . . . . . . 16

1.4.3 Maximin criterion . . . . . . . . . . . . . . . . . 16

1.5 Outline . . . . . . . . . . . . . . . . . . . 17

2 Optimization of blocked designs in fMRI studies $\quad 19$

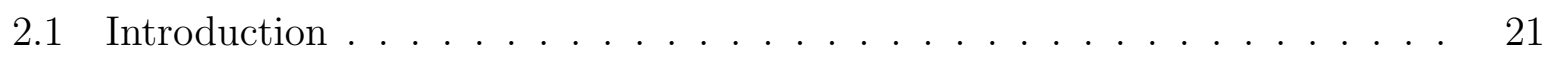

2.2 Model . . . . . . . . . . . . . . . . . . . . . . . . . . . 24

2.2.1 General linear model . . . . . . . . . . . . . . . . . . . . 24

2.2.2 Covariance structure . . . . . . . . . . . . . . . . 26

2.2.3 Checking of assumptions . . . . . . . . . . . . . . . . 27

2.3 Methods . . . . . . . . . . . . . . . . . . . . 28

2.3.1 Optimality criteria . . . . . . . . . . . . . . . . . 29

2.3.2 Maximin approach . . . . . . . . . . . . . . . . . . . 30

2.3.3 Search for locally optimal design . . . . . . . . . . . . . . . 30

2.3.4 Design factors . . . . . . . . . . . . . . . . . . . . . . . . . . . . . . . . . .

2.4 Results . . . . . . . . . . . . . . . . . . . . . . . 31 
2.4 .1 Autoregressive error of order 1 . . . . . . . . . . . . 31

2.4.2 Uncorrelated error and autoregressive error plus measurement error 35

2.5 Discussion . . . . . . . . . . . . . . . . . . . . 36

3 Robustness of optimal design of fMRI experiments with application of a $\begin{array}{lr}\text { genetic algorithm } & 39\end{array}$

3.1 Introduction . . . . . . . . . . . . . . . . . . . . . . . 41

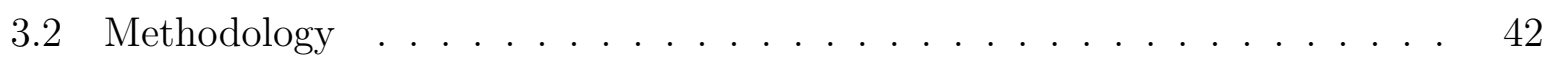

3.2.1 Linear models for estimation efficiency and detection power . . . . . 42

3.2.2 Design criteria and optimality criteria . . . . . . . . . . . . . . . . 43

3.2 .3 Maximin criterion . . . . . . . . . . . . . . . . . . . . . . . . . 44

3.2 .4 Simulations . . . . . . . . . . . . . . . . . . 45

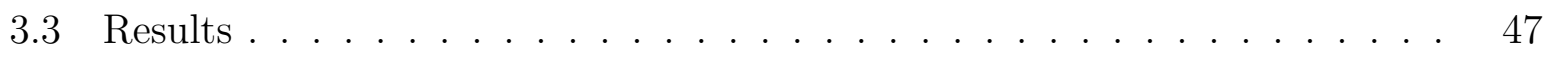

3.3.1 Optimal stimulus frequency . . . . . . . . . . . . . 47

3.3.2 Simulation Ia: Detection power for individual stimuli . . . . . . . . 47

3.3.3 Simulation Ib: Detection power for contrasts . . . . . . . . . . . 50

3.3 .4 Simulation II: Estimation efficiency . . . . . . . . . . . . . . . 50

3.3.5 Simulation III+IV: Multi-objective design criteria . . . . . . . . . 53

3.3.6 Comparison of $A$ - and $D$-optimality criterion . . . . . . . . . . . 55

3.4 Discussion and conclusions . . . . . . . . . . . . . . . . 56

3.A Derivation of optimal stimulus frequency for $D$-optimality criterion . . . . 58

3.A.1 Useful formulae . . . . . . . . . . . . . . . . . . . 58

3.A.2 Approximation of Fisher information matrix . . . . . . . . . 58

3.A.3 D-optimal stimulus frequency for estimation efficiency and detection power of individual stimulus effects . . . . . . . . . . . . 59

3.A.4 D-optimal stimulus frequency for estimation efficiency of contrasts and for detection power of contrasts . . . . . . . . . . . 60

3.A.5 Proof: Independence of $D$-optimal stimulus frequency from contrast matrix ......................... 61

4 Optimal design for nonlinear estimation of the hemodynamic response function

4.1 Introduction . . . . . . . . . . . . . . . . . . . . . . 65

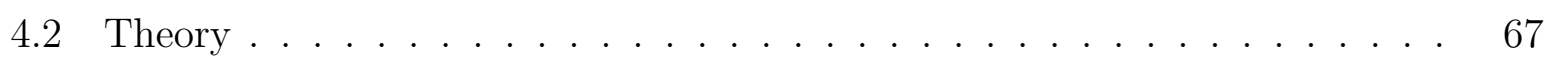

4.3 Methods . . . . . . . . . . . . . . . . . . . 71

4.3.1 Parameter space for expansion point . . . . . . . . . . . . . 71

4.3 .2 Design types . . . . . . . . . . . . . . . . . . . . . . . . . . . . . . . . . . . .

4.4 Results . . . . . . . . . . . . . . . . . . . . . . . . . . . . . . . . . . . .

4.4.1 Linearity of hemodynamic response function . . . . . . . . . . . 76

4.4.2 Nonlinearity of hemodynamic response function . . . . . . . . . . 81

4.4.3 Optimal designs for estimation efficiency and detection power . . . 82

4.5 Discussion . . . . . . . . . . . . . . . . . . . . . 83 
5 Optimal design of multi-subject blocked fMRI experiments $\quad 87$

5.1 Introduction . . . . . . . . . . . . . . . . . . . . 89

5.2 Methodology . . . . . . . . . . . . . . . . . 90

5.2 .1 Model and estimators . . . . . . . . . . . . . . . . . 90

5.2 .2 Optimality criterion and relative efficiency . . . . . . . . . . . 91

5.2 .3 Cost function . . . . . . . . . . . . . . . . . . . 93

5.2 .4 Numerical calculations . . . . . . . . . . . . . . . . . 94

5.3 Results . . . . . . . . . . . . . . . . . . . . . . . . 95

5.3.1 Optimal design for a given budget . . . . . . . . . . . . . 96

5.3.2 Effect of deviations from the optimal design for a given budget . . . 103

5.3.3 Optimal design for a given power . . . . . . . . . . . . . 105

5.4 Discussion and conclusions . . . . . . . . . . . . . . . 106

5.A Expression for $Z^{* T} Z^{*}$ in Section 5.2 .2 assuming uncorrelated errors . . . 110

5.A.1 General expression . . . . . . . . . . . . . . . . . 110

5.A.2 Approximated expression for matrix $M$ in Section 5.3.1.1 . . . . . . 112

5.B Calculation of $X^{* T} X^{*}$ in Eq. (5.29) . . . . . . . . . . . . . . 114

5.B.1 Calculation for one stimulus type and stimulus block length equal to null block length . . . . . . . . . . . . . . . . . . . . 114

5.B.2 Calculation for one stimulus and any block lengths . . . . . . . . 115

5.B.3 Calculation for any number of stimulus types . . . . . . . . . . 117

6 Summary and recommendations $\quad 119$

6.1 Summary . . . . . . . . . . . . . . . . . . . . . . . . . . . . . . . . . . . . . . . . 120

6.2 Guidelines . . . . . . . . . . . . . . . . . . . . . . . . . . 121

6.3 Ideas for future research . . . . . . . . . . . . . . . . . . 122

7 Samenvatting en richtlijnen (Dutch summary) 125

7.1 Samenvatting . . . . . . . . . . . . . . . . . . . . 126

7.2 Richtlijnen . . . . . . . . . . . . . . . . . . . . . . . . . . . . . . . . . . . 127

7.3 Ideeën voor toekomstig onderzoek . . . . . . . . . . . . . . . . . . . . . 129

$\begin{array}{ll}\text { References } & 131\end{array}$

$\begin{array}{ll}\text { Acknowledgments } & 141\end{array}$

$\begin{array}{ll}\text { About the author } & 143\end{array}$ 

CHAPTER 1

Introduction 


\subsection{Background to functional magnetic resonance imaging}

Since the first functional magnetic resonance imaging (fMRI) studies in the early 1990s (Belliveau et al., 1991; Ogawa et al., 1990; Kwong et al., 1992) functional magnetic resonance imaging (fMRI) has evolved quickly in its areas of application, methods of statistical analysis and experimental designs. Functional MRI provides a non-invasive method for researchers to study the functional areas of the human brain and can be used for a wide range of experimental questions because of its good balance between spatial and temporal resolution.

In early fMRI experiments, researchers employed blocked designs with long periods of continuous stimulus presentation as previously used for positron emission tomography (PET) (Amaro \& Barker, 2006; Culham, 2006), another neuroimaging method which has been invented in the 1980s. In blocked designs trials are presented in blocks of the same stimulus type with a short and constant time interval between two successive trials in a block (see Figure 1.1a). The time between offset of a trial and onset of the next trial is called interstimulus interval (ISI). Such a block is seen as a continuous sustained period of neuronal activation. The analysis method of these early blocked designs consisted of averaging the signal over all blocks of the same stimulus type (Buckner et al., 1996). However, it soon became clear that the fMRI technique allows for different designs and analysis methods making use of the higher temporal resolution of fMRI in comparison to PET and the ability of fMRI to measure the response to single trials (Buckner et al., 1996). Researchers started using event-related designs which make use of interstimulus intervals (ISIs) from mostly 2 to $20 \mathrm{~s}$, a random order of trial types and jittered ISIs (see Figure 1.1b). Jittering of ISIs means that the ISI is randomized. Event-related designs are seen as triggering short and discrete neuronal activity in contrast to the sustained neuronal activity of blocked designs.

Nowadays, blocked and event-related designs are the main types of designs which are employed in fMRI experiments. The purpose of this thesis is to optimize these design types to obtain precise estimates with small variance or a small confidence ellipsoid. Hereby, it is important to take the purpose of an fMRI experiment and its costs into consideration. One important question is the optimal combination of number of subjects and number of time points per subject in an experiment with a limited budget. On the subject level the ordering and spacing of the trials is a critical aspect for optimization of an experiment. Furthermore, psychological aspects play a role, e.g., unpredictability of a stimulus presentation sequence is one desirable property of a design. Otherwise, subjects will predict the sequence and react with non-task-related activity. The optimization of experimental fMRI design is complicated by the noise structure of fMRI data (Lindquist, 2008). Most studies on optimal fMRI experiments considered uncorrelated noise but the inclusion of correlated noise will influence the optimal design (Lindquist, 2008). In this thesis optimal designs for different error models and different values for the error parameters are considered. So far, the optimization of fMRI experiments has not much benefited from the methods which are already available and established in optimal design theory. Newly introduced methods to 
Stimulus type A

Block length

Stimulus type B

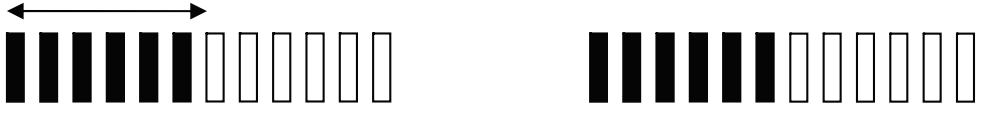

time (s)

(a) Blocked design

Stimulus type A

Stimulus type B

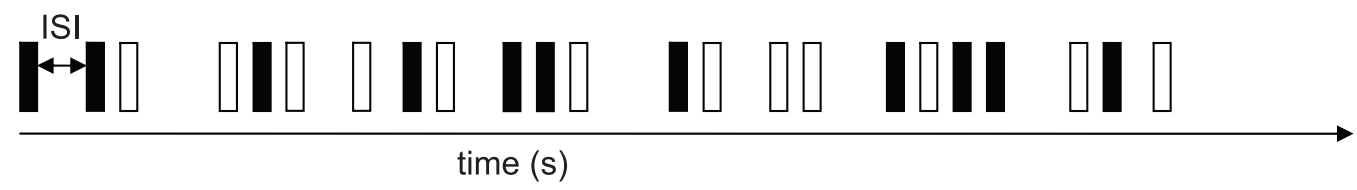

(b) Event-related design

Figure 1.1: Blocked and event-related design with two stimulus types. Block length and interstimulus interval (ISI) are illustrated.

fMRI design optimization in this thesis are for example the $D$-optimality criterion, the concept of local optimality and the maximin criterion. It is relevant for optimization of experimental design to know how the data from an experiment are obtained and analyzed.

During an fMRI experiment, the subject lies in the scanner and the magnetic field which is generated by the scanner while performing a task, e.g., finger-tapping, inner speech or passive viewing. The measured fMRI signal in most studies is based on the different magnetic properties of deoxygenated and oxygenated blood. Changes in the fMRI signal are caused by the varying concentration of deoxygenated and oxygenated blood in activated brain regions (Bandettini \& Underleider, 2001). The blood-oxygenlevel-dependent (BOLD) response is an indirect measure of neuronal activity (Logothetis \& Wandell, 2004). The function which describes the measured BOLD response to one short stimulus is called the hemodynamic response function (HRF). In contrast to the short spike responses of active neurons the hemodynamic response function evolves over a period of $30 \mathrm{~s}$ (see Figure 1.2).

When several trials of a stimulus type are presented successively with a short ISI, the hemodynamic responses to these trials overlap and add up to the final response to this stimulus type. This property of the hemodynamic response is called superposition or linearity of the HRF. Mathematically it can be described by convolution of the stimulus sequence, a sequence of 0 and 1 indicating the presence of a stimulus type at a given time point, with the hemodynamic response function (see Figure 1.3). The difference of two gamma functions is often used as standard HRF in fMRI data analysis. Nonlinearity of the HRF has been observed for smaller ISIs so that very small ISIs, e.g., 1 s, should be avoided or nonlinearity should be taken care of.

The most often used model to analyze fMRI data from one subject is nowadays the 


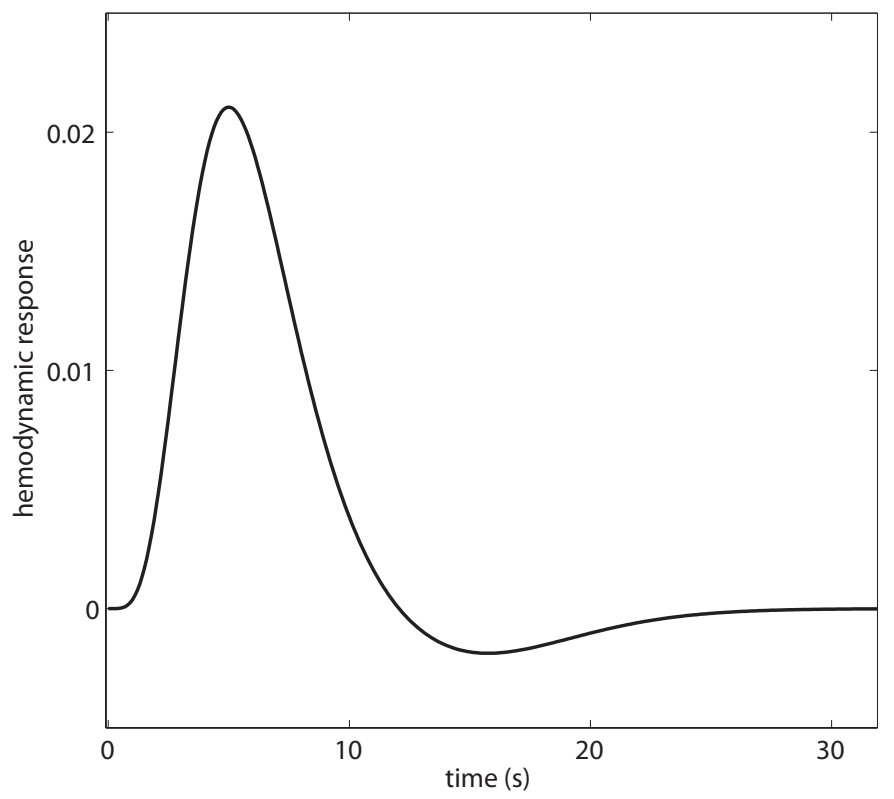

Figure 1.2: Hemodynamic response function modeled by a double gamma function

general linear model (GLM) (Lindquist, 2008). It can be differentiated between two types of models, that is a model for estimation of the HRF and a model for detection of activation. For the former type the height of the HRF at different time points over its course is estimated. The latter type is only interested in the amplitude parameter associated with the convolved time series obtained by convolution of the stimulus sequence with a standard HRF (see Figure 1.3). It has been found that blocked designs are optimal for detection of activation, also called detection power, whereas event-related designs are optimal for estimation of the HRF, also called estimation efficiency (Liu et al., 2001; Liu \& Frank, 2004; Birn et al., 2002).

The fMRI signal is sampled spatially and temporally. At each time point a scan is performed either over a region of interest (ROI) or the whole brain. For example for whole brain coverage, the data is obtained in several slices of the brain and each slice consists itself of several voxels. Typically, a slice can contain $64 \times 64$ voxels, where a voxel itself is a small imaging unit for instance of $3 \mathrm{~mm} \times 3 \mathrm{~mm} \times 3 \mathrm{~mm}$. The set of all slices is called a volume and a volume can consist of 30 slices for whole brain coverage. During the time course of one continuous period of scanning called a run, several volumes, e.g., 200 - 300, are obtained at the time points of the experiment. This presents an enormous amount of data with around 100,000 voxels per subject and per voxel a time course of 200 - 300 time points. For the GLM a mass univariate approach is employed which fits one GLM at each voxel. A statistical test can then be performed on the estimated effects of interest at each voxel taking the multiple testing problem into account. Several methods are employed to handle the multiple testing problem in the GLM approach, e.g., Gaussian random field theory (Worsley et al., 1996) and false-discovery rate (Genovese et al., 2002). The focus in this thesis is on models for one voxel and determination of optimal designs for the precise estimation of the effects with such a model. This optimal design will improve 

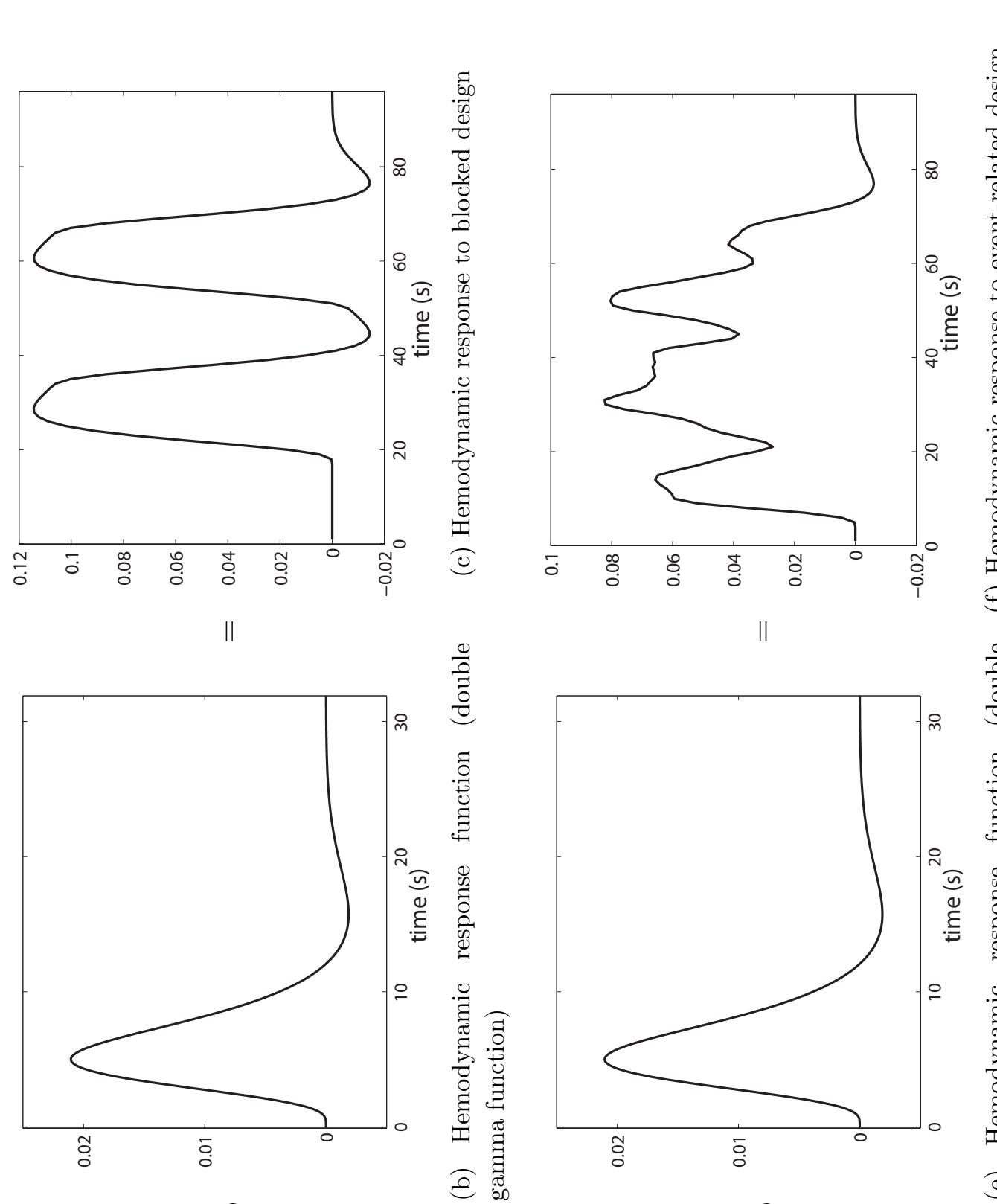

$\otimes$

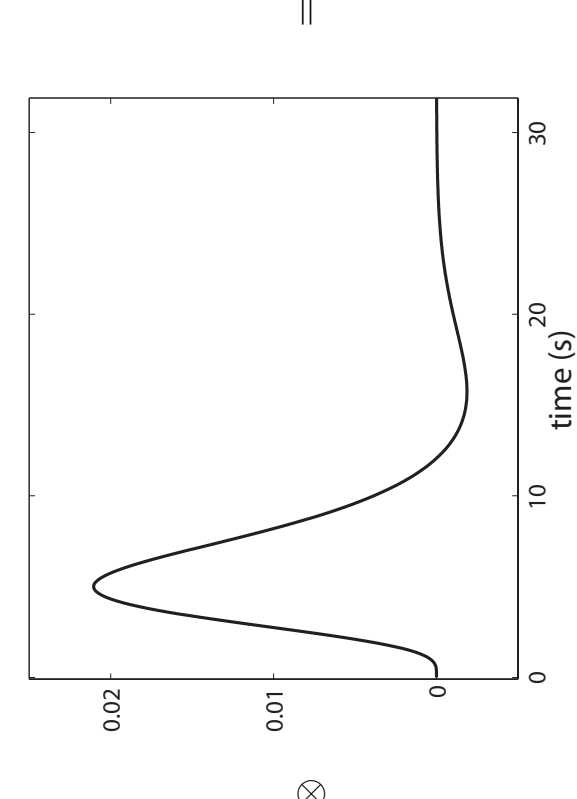

$\otimes$

Q

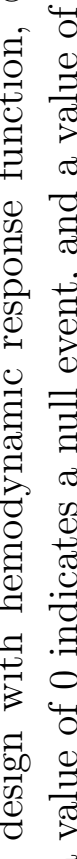

임

¿ $\quad \stackrel{0}{ \pm}$

苛

छ d

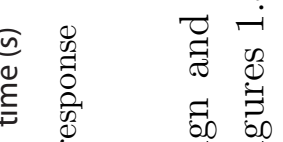

$-\quad \frac{60}{6}$

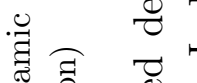

焉兽

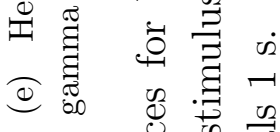

कै

过
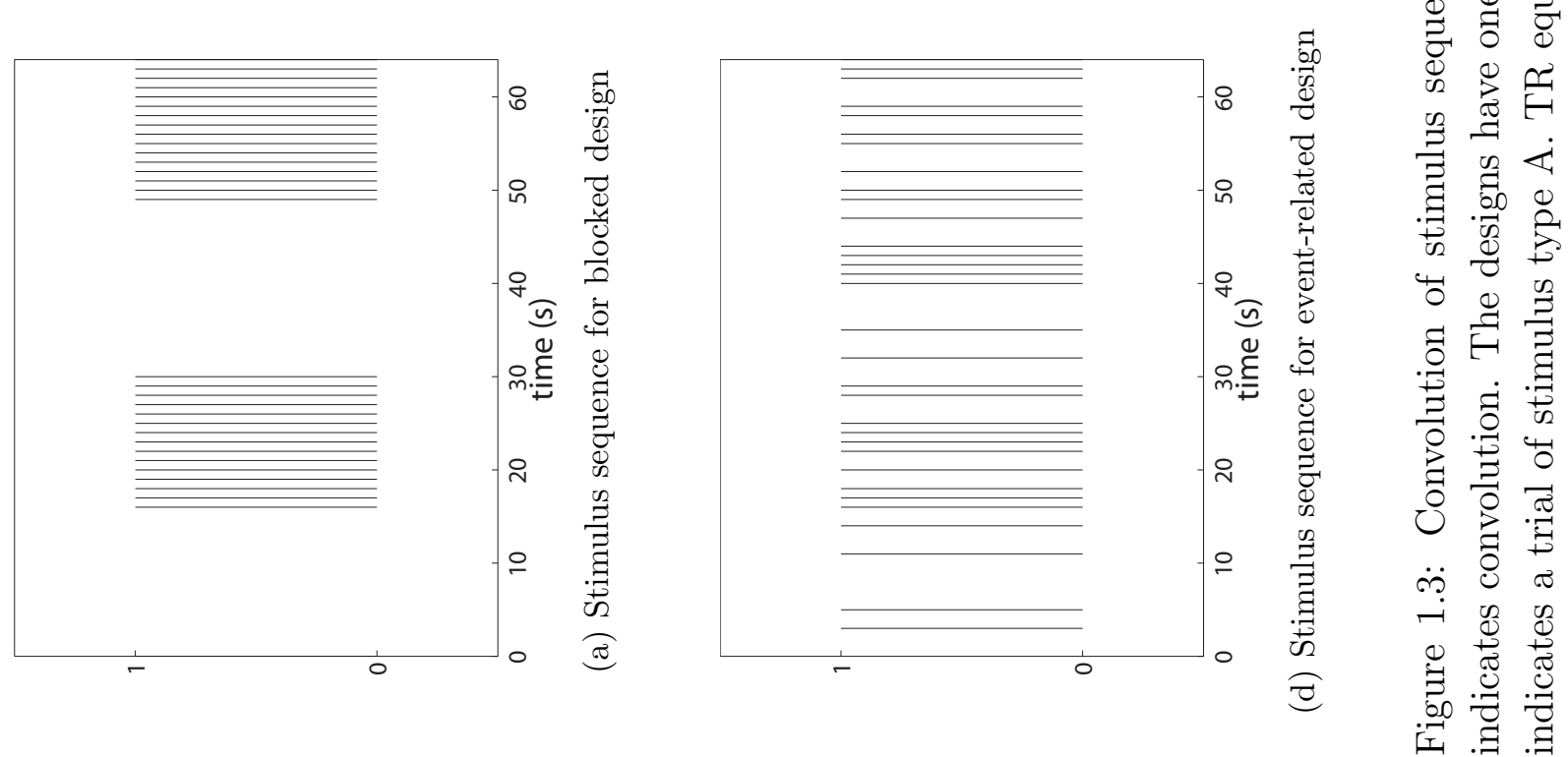
the estimation of the effects in all single-voxel analyses in the GLM approach.

In Section 1.2 experimental designs for fMRI studies are described in more detail. An example of a blocked design and of an event-related design are given. Four different models used in this thesis are explained in Section 1.3. These models can be employed to estimate the HRF linearly (Model 1, GLM type 1), detect activation (Model 2, GLM type 2), estimate the HRF nonlinearly (Model 3) and detect activation in a multi-subject fMRI experiment (Model 4). Section 1.4 presents the employed criteria and methods to find optimal designs, i.e., the optimality criteria and the maximin criterion to handle local optimality. Finally, an outline of the thesis is given in Section 1.5.

\subsection{Design of fMRI experiments}

First a notation for an fMRI design will be presented and some general aspects will be explained before blocked and event-related designs will be discussed separately. A design with $Q=3$ stimulus types can be denoted by

$$
\xi=\left\{\begin{array}{llllllll}
1 & 0 & 2 & 3 & \ldots & 2 & 3 & 0
\end{array}\right\}
$$

where the numbers 1 to 3 present the different stimulus types, also called conditions. The 0 stands for null events, where no stimulus or a simple control task are presented, e.g., fixation, where the subject views a fixation cross on a screen. These simple control tasks are also called baseline conditions as the baseline of the fMRI signal is measured during these conditions. The choice of appropriate control tasks is important because the comparison between the stimulus task and the control task serves to isolate the cognitive process of interest (Chee et al., 2003; Donaldson \& Buckner, 2001; Culham, 2006).

The time between two successive events, including null events, in the sequence $\xi$ is given by the greatest common divider of all employed stimulus onset asynchronies. The stimulus onset ansychrony (SOA) is the sum of stimulus duration and interstimulus interval. For example, if SOAs $2 \mathrm{~s}, 3 \mathrm{~s}$ and $4 \mathrm{~s}$ are employed, the greatest common divider is $1 \mathrm{~s}$ and gives the time between elements in the sequence $\xi$. Stimulus types with very short duration are seen as events with no stimulus duration and the ISI is then equal to SOA. For most designs in this thesis the time between two successive events, including null events, in $\xi$ will be for simplicity equal to the repetition time (TR), the time between two scans. Instead of numbers, the stimulus types can also be denoted by letters, e.g., A, B or C for three stimulus types. The designation of the different stimulus types is arbitrary and the numbers 1, 2 and 3 or letters A, B and $\mathrm{C}$ do not refer to any type of order.

In the following, blocked and event-related design types will be discussed separately. Both design types have their advantages and disadvantages and are equally useful. The interested reader is referred to the literature for a review of advantages and disadvantages (Chein \& Schneider, 2003; Aguirre \& D'Esposito, 1999; Amaro \& Barker, 2006). The factors, which describe a blocked or an event-related design and which have to be considered for optimization, are introduced in the following subsections. Another factor which can also be taken into account for blocked designs as well as event-related designs, but is not 
part of the optimization in this thesis, is the stimulus duration (Bandettini \& Cox, 2000; Birn et al., 2002) which is assumed for simplicity to be fixed by the fMRI researcher.

\subsubsection{Blocked designs}

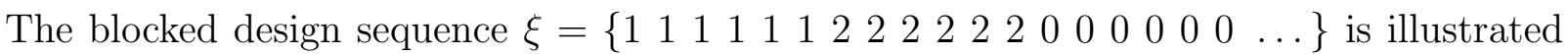
in Figure 1.1a. The study of Buckner et al. (1996) gives an example of a blocked design. Word stems, such as 'ope-' or 'fut-', were presented to subjects who were instructed to mentally complete these stems. Buckner et al. (1996) performed blocked designs where the stems were presented to each subject every $2.5 \mathrm{~s}$ within $30 \mathrm{~s}$ blocks alternated with $30 \mathrm{~s}$ of rest. This example illustrates the relevant factors determining a design sequence for a blocked design with fixed total experimental time: the block length, the ordering of blocks and the time between two successive trials in a block (SOA). The number of blocks or number of trials in a block are given by these three factors because of the fixed total time and are therefore not relevant for characterization of a blocked design. For optimization of a blocked design sequence these three factors will thus be considered. The block order indicates that this pattern is repeated several times during an experiment, i.e., a block order of $\mathrm{AB}$, where $\mathrm{A}$ and $\mathrm{B}$ denote different block types, results in several repetitions of AB: ABABABAB.

Typical block lengths are in the range of $10 \mathrm{~s}$ to $60 \mathrm{~s}$. Longer block lengths are not reasonable to avoid habituation or fatigue of the subject. Furthermore, longer block lengths result in lower frequency of the design and will interfere with the low frequency noise in the fMRI signal. The SOA depends on the stimulus and task type but is normally short. At least two conditions have to be considered, i.e., a control condition and a task condition. A simple blocked design is the so called alternating boxcar design (Chein \& Schneider, 2003) or "AB block" (Amaro \& Barker, 2006) design with two alternating conditions A and $\mathrm{B}$ repeated and compared in the final analysis. Stimulus blocks will be denoted by $\mathrm{A}, \mathrm{B}, \ldots$ and null blocks (rest or baseline condition) by N. The block orders considered in this thesis for two or three conditions are $\mathrm{AB}$, ANBN with stimulus blocks alternating with null blocks, or ABN with null blocks happening equally often as stimulus blocks (see Figure 1.1a).

\subsubsection{Event-related designs}

Event-related designs are categorized into slow event-related designs and rapid eventrelated designs. Slow event-related designs use a fixed interstimulus interval of $10 \mathrm{~s}$ to 16 s so that the main part of the BOLD signal has evolved during the ISI (see Figure 1.2). For slow event-related designs the fixed ISI has been a subject of optimization (Bandettini \& Cox, 2000). It has been shown that for a slow event-related design with one stimulus type an ISI of 12 - $14 \mathrm{~s}$ is optimal for a stimulus duration of $2 \mathrm{~s}$ or less (Bandettini \& Cox, 2000).

The study of Buckner et al. (1996) also gives an example of a slow event-related design with a fixed ISI of about $15 \mathrm{~s}$. The task which subjects had to perform was the same as described for the blocked design, i.e., mental completion of word stems. Buckner et al. 
(1996) found similar patterns of activation for the blocked and event-related design. This result was seen as a validation of event-related designs. They further demonstrated how the results of the event-related design can be used to gain further information about the timing of the BOLD response.

The next step in the development of event-related designs was taken among others by Burock et al. (1998) who compared event-related designs with fixed ISI to rapid eventrelated designs and found in simulations that rapid event-related designs with randomized ISIs allow for much faster presentation rates than the slow event-related design with fixed ISI. Their empirical study offers an example of a rapid event-related design. They used a randomization interval for the SOA of $500 \mathrm{~ms}$ and presented two different stimulus types and null events with equal probability at each interval to two subjects. The two stimulus types and the null event were left-hemifield checkerboard, right-hemifield checkerboard and fixation. In rapid event-related designs, null events are relevant to generate the jittering of SOAs which is necessary to generate some variation in the hemodynamic response and in the design frequency. This variation in the hemodynamic response enables HRF estimation via the general linear model (Miezin et al., 2000). An example of a rapid event-related design is shown in Figure 1.1b.

The design of Burock et al. (1998) is an example of a possible way to characterize eventrelated designs. Rapid event-related designs can be described by the minimum possible SOA between trials $\left(\mathrm{SOA}_{\text {min }}\right)$ and the probability $P$ of a stimulus type or a null event to be presented (stationary stochastic designs in Friston et al. (1999) and Zarahn \& Friston (2002)). For this characterization of a rapid-event related design the $\mathrm{SOA}_{\min }$ and the probability $P$ are factors of optimization. The SOA will be geometrically distributed with values which are multiples of the $\mathrm{SOA}_{\text {min }}$ and the trial order will be completely random. However, the fact that this characterization results only in SOAs which are multiples of the $\mathrm{SOA}_{\text {min }}$ might be a restriction if there is a lower limit higher than $0 \mathrm{~s}$ for the minimum SOA. Such a lower limit can be given by the stimulus duration or the need to avoid nonlinearity of the HRF caused by low SOAs.

Other ways to categorize rapid event-related are also possible. For example, Hagberg et al. (2001) considered rapid event-related designs with one stimulus type and with different distributions for ISIs, e.g., a uniform distribution for ISIs of $2 \mathrm{~s}, 3 \mathrm{~s}, \ldots, 8 \mathrm{~s}$ where each ISI has the same probability. Different trial orders, i.e., random, alternating and permuted (pseudo-random), are considered by Josephs \& Henson (1999). These different trial orders are described by the $\mathrm{SOA}_{\text {min }}$ and a transition matrix which gives the occurrence probability of a stimulus type conditional on the previous $m$ trials. Friston et al. (1999) also studied non-stationary stochastic designs where the occurrence probability varies per time point. Especially the optimization of rapid event-related designs is complicated by the enormous amount of possible fMRI designs and the different possibilities to describe rapid event-related designs. 


\subsection{Models}

All presented models describe the time series for one voxel. The number of time points for the experiment and the number of stimulus types will be denoted by $T$ and $Q$. The number of sampling time points for the HRF is given by $k$. The optimal design will depend on the relevant model for the data analysis. The generalized least squares (GLS) estimator for the unknown parameters of interest is determined from the following models assuming that the error covariance matrix $\Sigma$ is known. In practice however the covariance matrix $\Sigma$ and its parameters will be unknown and can be estimated using maximum likelihood (ML) or restricted maximum likelihood methods (REML) (Diggle et al., 1994). As the error is assumed to be normally distributed, the GLS estimator for the unknown parameters of interest equals the ML estimator which is obtained by maximizing the likelihood function.

\subsubsection{Model 1: Linear estimation of hemodynamic response func- tion}

The general linear model (GLM) is commonly employed for the analysis of fMRI experimental data from one subject. The measured fMRI signal $Y$ is a vector of form $T \times 1$ and is described by

$$
Y=X h+S \gamma+\epsilon
$$

where the design matrix for the parameter vector $h$ is given by the $T \times(Q k)$ matrix $X$. The $(Q k) \times 1$ vector $h=\left(h_{1}^{T}, \ldots, h_{Q}^{T}\right)^{T}$ is a vector of the $Q$ hemodynamic response functions $h_{q}$ which are each sampled at $k$ time points. The entries in vector $h_{q}=\left(h_{q 1}, \ldots, h_{q k}\right)^{T}$ represent the heights of the hemodynamic response function of stimulus type $q$ at time points $1, \ldots, k$ (see Figure 1.4). The nuisance matrix $S$ models nuisance terms, e.g., scanner drift, and $\gamma$ is the nuisance parameter. The intercept is part of the nuisance parameter $\gamma$. The error $\epsilon$ is multivariate normally distributed with expectation 0 and covariance matrix $\Sigma$.

The matrix $X=\left[\begin{array}{lll}X^{1} & \ldots & X^{Q}\end{array}\right]$ is a concatenation of the different stimulus convolution matrices $X^{q}$ for each stimulus type. Each column of the binary matrix $X^{q}$ is linked to one parameter of the HRF parameter vector $h_{q}$. The vector $h_{q}$ represents the hemodynamic response function for stimulus type $q$ sampled discretely over a time period of for instance $32 \mathrm{~s}$. The sampling rate of the HRF depends on the relation of employed SOAs and TR. When all SOAs are multiples of TR, the sampling rate of the hemodynamic response function equals TR. A systematic variation of the relation between the image acquisition and the trial presentation is achieved by SOAs which are not multiples of TR. Such a variation results in a higher sampling rate than TR so that the temporal course of the HRF is estimated at more time points (Josephs et al., 1997; Miezin et al., 2000). The sampling rate of the hemodynamic response function will be denoted by $\Delta t$.

As illustration, the matrix $X$ will be presented for an example design $\xi=\left\{\begin{array}{llll}1 & 1 & 0 & 2\end{array}\right.$ $\left.\begin{array}{lll}1 & 0 & \ldots\end{array}\right\}$ with two stimulus types. For simplification the focus is on SOAs which are multiples of TR. The time between two entries in $\xi$ is then given by TR. The stimulus sequence for stimulus type 1 is $s^{1}=\left\{\begin{array}{lllllll}1 & 1 & 0 & 0 & 1 & 0 & \ldots\end{array}\right\}$ and the stimulus sequence 


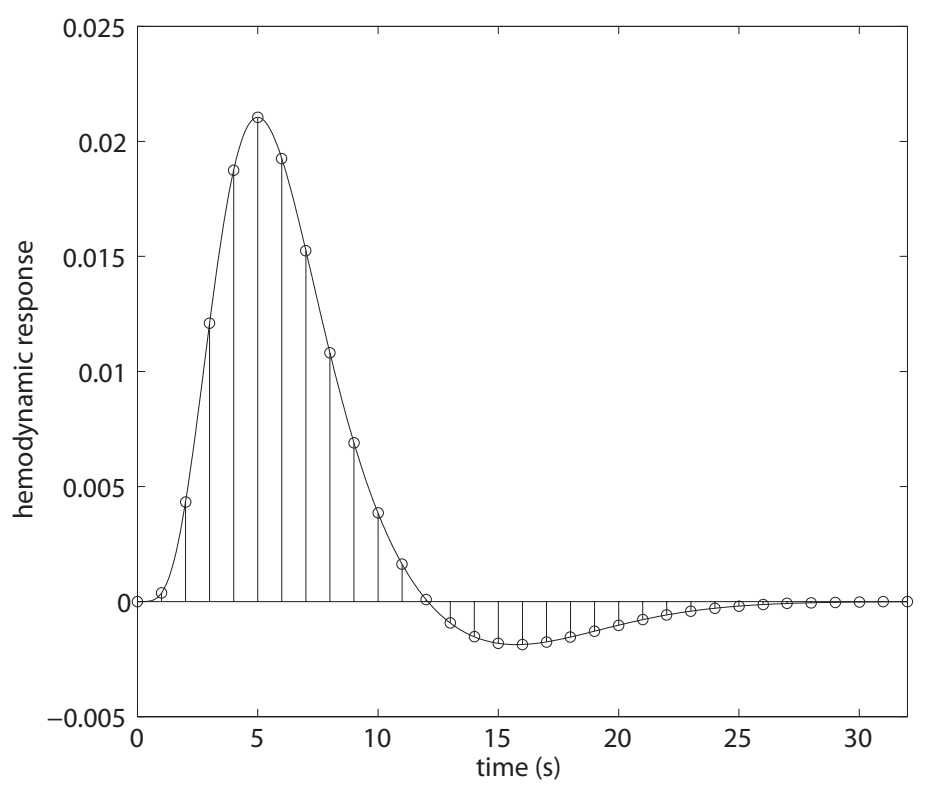

Figure 1.4: Sampling of hemodynamic response function $h_{q}$ with rate $1 \mathrm{~s}$ and $k=33$ time points over a time period of $32 \mathrm{~s}$. Parameters $h_{q 1}, \ldots, h_{q 33}$ give the unknown heights of the hemodynamic response function at time points 1 (0 s), ., 33 (32 s).

for stimulus type 2 is given by $s^{2}=\left\{\begin{array}{lllllll}0 & 0 & 0 & 1 & 0 & 0 & \ldots\end{array}\right\}$. The HRFs for both stimulus types are assumed to be sampled over $10 \mathrm{~s}$ with $\mathrm{TR}=\Delta t=2 \mathrm{~s}$ so that $k=6$. The matrix $X^{1}$ and $X^{2}$ have the following forms

$\begin{array}{cccccccccccccc} & 0 \mathrm{~s} & 2 \mathrm{~s} & 4 \mathrm{~s} & 6 \mathrm{~s} & 8 \mathrm{~s} & 10 \mathrm{~s} & & 0 \mathrm{~s} & 2 \mathrm{~s} & 4 \mathrm{~s} & 6 \mathrm{~s} & 8 \mathrm{~s} & 10 \mathrm{~s} \\ 2 \mathrm{~s} & 1 & 0 & 0 & 0 & 0 & 0 & 2 \mathrm{~s} & 0 & 0 & 0 & 0 & 0 & 0 \\ 4 \mathrm{~s} & 1 & 1 & 0 & 0 & 0 & 0 & 4 \mathrm{~s} & 0 & 0 & 0 & 0 & 0 & 0 \\ 6 \mathrm{~s} & 0 & 1 & 1 & 0 & 0 & 0 & 6 \mathrm{~s} & 0 & 0 & 0 & 0 & 0 & 0 \\ 8 \mathrm{~s} & 0 & 0 & 1 & 1 & 0 & 0 & 8 \mathrm{~s} & 1 & 0 & 0 & 0 & 0 & 0 \\ 10 \mathrm{~s} & 1 & 0 & 0 & 1 & 1 & 0 & 10 \mathrm{~s} & 0 & 1 & 0 & 0 & 0 & 0 \\ 12 \mathrm{~s} & 0 & 1 & 0 & 0 & 1 & 1 & 12 \mathrm{~s} & 0 & 0 & 1 & 0 & 0 & 0 \\ & \vdots & \vdots & \vdots & \vdots & \vdots & \vdots & & \vdots & \vdots & \vdots & \vdots & \vdots & \vdots\end{array}$

The first column of $X^{q}$ gives the stimulus sequence $s^{q}$ for stimulus type $q$ :

$$
s^{q}(t)= \begin{cases}1 & \text { if stimulus } q \text { at time point } t \\ 0 & \text { if no stimulus } q \text { at time point } t\end{cases}
$$

The other columns are shifted versions of the stimulus sequences. Each row of the matrices $X^{1}$ and $X^{2}$ refers to one measurement time point of the fMRI signal (one scan) which are multiples of TR and each column refers to one sampling time point of the HRF. In general, all entries of the matrix $X^{q}$ are given by the following equation:

$$
X_{i j}^{q}= \begin{cases}1 & \text { if there is a stimulus } q \text { at time point } i \cdot \mathrm{TR}-(j-1) \cdot \Delta t \\ 0 & \text { otherwise. }\end{cases}
$$


Multiplication of the stimulus convolution matrix $X^{q}$ with $h_{q}$ equals discrete convolution of the stimulus sequence $s^{q}$ with $h_{q}$, thus $X^{q} h_{q}=s^{q} \otimes h_{q}$.

The generalized least squares estimator minimizes the sum of the generalized least squares (Seber, 1977, p.60-62), here $\left(Y-\left[\begin{array}{l}X S\end{array}\right]\left(h^{T} \gamma^{T}\right)^{T}\right)^{T} \Sigma^{-1}\left(Y-\left[\begin{array}{ll}X & S\end{array}\right]\left(h^{T} \gamma^{T}\right)^{T}\right)$. For $\left(h^{T} \gamma^{T}\right)^{T}$, the generalized least squares estimator equals

$$
\widehat{\left(\begin{array}{l}
h \\
\gamma
\end{array}\right)}=\left(\left[\begin{array}{ll}
X & S
\end{array}\right]^{T} \Sigma^{-1}\left[\begin{array}{ll}
X & S
\end{array}\right]\right)^{-1}\left[\begin{array}{ll}
X & S
\end{array}\right]^{T} \Sigma^{-1} Y .
$$

Interest is mainly in the parameter vector $h$ and the GLS estimator for $h$ is given by

$$
\hat{h}=\left(X^{T} V^{T}\left(I-P_{V S}\right) V X\right)^{-1} X^{T} V^{T}\left(I-P_{V S}\right) V Y,
$$

where $P_{V S}=V S\left((V S)^{T} V S\right)^{-1}(V S)^{T}$ is the projection matrix onto the vector space spanned by the columns of the matrix $V S$ and $V$ is the square root matrix of the inverse error covariance matrix $\Sigma^{-1}$. This can be seen using Theorem 8.5.11 for the inverse of partitioned matrices in Harville (1997) or from Seber (1977, p.65-66). The $(Q k) \times(Q k)$ covariance matrix $\operatorname{Cov}(\hat{h})$ equals

$$
\operatorname{Cov}(\hat{h})=\left(X^{T} V^{T}\left(I-P_{V S}\right) V X\right)^{-1} .
$$

\subsubsection{Model 2: Detection}

For detection fixed hemodynamic response functions for all stimulus types are assumed and the interest is in estimation of the HRF amplitude for a given stimulus type. The amplitude in relation to its estimated variance is a measure of the strength of the activation and it will also be spoken of stimulus effect instead of amplitude. A commonly employed model for the HRF is the double gamma function, a difference of two gamma functions, with parameter vector $\theta=\left(a_{1}, b_{1}, c, a_{2}, b_{2}\right)$ :

$$
h(t, \theta)=\frac{b_{1}^{a_{1}+1} t^{a_{1}} \exp \left(-b_{1} t\right)}{\Gamma\left(a_{1}+1\right)}-\frac{b_{2}^{a_{2}+1} t^{a_{2}} \exp \left(-b_{2} t\right)}{c \Gamma\left(a_{2}+1\right)},
$$

where $t$ is time in seconds. The first gamma function models the peak of the hemodynamic response function and the second gamma function models the undershoot of the HRF. In Figure 1.2 the canonical double gamma function, which has the parameter values $a_{1}=5$, $b_{1}=1, c=6, a_{2}=15$ and $b_{2}=1$ for Eq. (1.9), is shown. The model for detection is as follows

$$
Y=Z \beta+S \gamma+\epsilon
$$

where the design matrix $Z=X H_{0}$ is obtained by multiplication of $X$ in Eq. (1.2) with the matrix $H_{0}$. The matrix $H_{0}$ is a $(Q k) \times Q$ block matrix with the $k \times 1$ hemodynamic response vectors $h_{0}^{q}$ of the different stimulus types as blocks. The unknown parameter vector $\beta=\left(\beta_{1}, \ldots, \beta_{Q}\right)^{T}$ represents the amplitudes of the hemodynamic responses for the different stimulus types. As before, $S$ is a nuisance matrix modeling for instance scanner 
drift, $\gamma$ is the nuisance parameter and $\epsilon$ is a normally distributed error with expectation 0 and covariance matrix $\Sigma$.

In this thesis it is assumed that each stimulus type has the same hemodynamic response function, thus $h_{0}^{q_{1}}=h_{0}^{q_{2}}$ for all $q_{1}, q_{2}=1, \ldots, Q$ and only the amplitudes $\beta_{q}$ can vary per stimulus type. This is a common assumption in fMRI data analysis. The common hemodynamic response vector is denoted by $h_{0}$ and it holds then that $Z=X\left(I_{Q} \otimes_{K} h_{0}\right)$ where $\otimes_{K}$ denotes the Kronecker product. Furthermore, it is assumed that this hemodynamic response vector $h_{0}$ is obtained by sampling the canonical double gamma function with values in Eq. (1.9) as given above. The matrix $Z=\left[X^{1} h_{0} \ldots X^{Q} h_{0}\right]$ has in each column the hemodynamic response $X^{q} h_{0}$ (without amplitude $\beta_{q}$ ) to one stimulus type. This hemodynamic response $X^{q} h_{0}$ equals the convolution of the stimulus sequence for stimulus type $q$ with the fixed hemodynamic response vector $h_{0}$. In Figures $1.3 \mathrm{c}$ and $1.3 \mathrm{f} Z$ is shown for a blocked design and an event-related design with one stimulus type. The entries in the vector $Z$ equal the heights of the hemodynamic response at the different time points.

For $\left(\beta^{T} \gamma^{T}\right)^{T}$, the GLS estimator has the following expression

$$
\widehat{\left(\begin{array}{l}
\beta \\
\gamma
\end{array}\right)}=\left([Z S]^{T} \Sigma^{-1}[Z S]\right)^{-1}[Z S]^{T} \Sigma^{-1} Y .
$$

The GLS estimator for $\beta$ and its $Q \times Q$ covariance matrix are given by

$$
\begin{aligned}
\hat{\beta} & =\left(Z^{T} V^{T}\left(I-P_{V S}\right) V Z\right)^{-1} Z^{T} V^{T}\left(I-P_{V S}\right) V Y, \\
\operatorname{Cov}(\hat{\beta}) & =\left(Z^{T} V^{T}\left(I-P_{V S}\right) V Z\right)^{-1} .
\end{aligned}
$$

\subsubsection{Model 3: Nonlinear estimation of hemodynamic response function}

The hemodynamic response function can vary between subjects and between regions in one subject (Aguirre et al., 1998; Miezin et al., 2000; Handwerker et al., 2004). This means that for the double gamma function as HRF model different parameters $\theta$ would have to be employed. In the following a nonlinear model for the HRF is developed based on the commonly employed double gamma function. This model can be used to estimate subjectspecific or region-specific parameters for the double gamma HRF. Only one stimulus type is considered but the estimated specific parameters can be used in subsequent detection analyses with more stimulus types. The model is

$$
Y=X h(\theta) \beta+S \gamma+\epsilon
$$

with $X$ being the binary matrix as described above and $h(\theta)$ the double gamma function for parameter vector $\theta$ sampled at $k$ time points. The parameter $\beta$ represents the amplitude of the HRF. As above, the nuisance matrix $S$ models scanner drift, $\gamma$ is the unknown nuisance parameter and $\epsilon$ represents normally distributed error with mean 0 and covariance matrix $\Sigma$. 
The nonlinear model in Eq. (1.14) is linearized by a first-order Taylor approximation of $h$ with respect to $\theta=\left(a_{1}, b_{1}, c, a_{2}, b_{2}\right)$ :

$$
h(t, \theta) \approx h\left(t, \theta_{0}\right)+\left.\sum_{j=1}^{5} \frac{\partial h(t, \theta)}{\partial \theta_{j}}\right|_{\theta=\theta_{0}}\left(\theta_{j}-\theta_{0, j}\right),
$$

where $\theta_{j}$ gives the $j$ th entry in $\theta$, e.g., $\theta_{1}=a_{1}$, and $\theta_{0, j}$ gives the $j$ th entry in $\theta_{0}$. Substituting Eq. (1.15) into Eq. (1.14), the following model is obtained:

$$
Y \approx X H\left(\theta_{0}\right) \tau+S \gamma+\epsilon
$$

where the matrix $H\left(\theta_{0}\right)$ consists of the double gamma function and its derivatives evaluated at the Taylor expansion point $\theta_{0}$ and sampled at time points $t=1, \ldots, k$ :

$$
H\left(\theta_{0}\right)=\left(\begin{array}{cccc}
h\left(1, \theta_{0}\right) & \left.\frac{\partial h(1, \theta)}{\partial \theta_{1}}\right|_{\theta=\theta_{0}} & \ldots & \left.\frac{\partial h(1, \theta)}{\partial \theta_{5}}\right|_{\theta=\theta_{0}} \\
h\left(2, \theta_{0}\right) & \left.\frac{\partial h(2, \theta)}{\partial \theta_{1}}\right|_{\theta=\theta_{0}} & \ldots & \left.\frac{\partial h(2, \theta)}{\partial \theta_{5}}\right|_{\theta=\theta_{0}} \\
\vdots & & & \vdots \\
h\left(k, \theta_{0}\right) & \left.\frac{\partial h(k, \theta)}{\partial \theta_{1}}\right|_{\theta=\theta_{0}} & \ldots & \left.\frac{\partial h(k, \theta)}{\partial \theta_{5}}\right|_{\theta=\theta_{0}}
\end{array}\right),
$$

and $\tau=\left(\beta, \beta\left(\theta_{1}-\theta_{0,1}\right), \ldots, \beta\left(\theta_{5}-\theta_{0,5}\right)\right)^{T}$.

The GLS estimator for $\tau$ is given by

$$
\hat{\tau}=\left(H\left(\theta_{0}\right)^{T} X^{T} V^{T}\left(I-P_{V S}\right) V X H\left(\theta_{0}\right)\right)^{-1} H\left(\theta_{0}\right)^{T} X^{T} V^{T}\left(I-P_{V S}\right) V Y
$$

with covariance matrix

$$
\operatorname{Cov}(\hat{\tau})=\left(H\left(\theta_{0}\right)^{T} X^{T} V^{T}\left(I-P_{V S}\right) V X H\left(\theta_{0}\right)\right)^{-1}
$$

\subsubsection{Model 4: Mixed-effects model for detection}

So far, all models were for a single-subject analysis. For the analysis of multi-subject data a linear mixed-effects model will be presented. For a multi-subject analysis the brain volume from each subject is normalized into a standard stereotactic space, e.g., the Talairach and Tourneaux brain or Montreal Neurological Institute (MNI) brain. The model for subject $i$ is given by

$$
\begin{aligned}
Y_{i} & =Z \beta+Z b_{i}+S \gamma_{i}+\epsilon_{i} \\
& =\left[\begin{array}{ll}
Z & S
\end{array}\right]\left(\begin{array}{ll}
\beta^{T} & \gamma_{i}^{T}
\end{array}\right)^{T}+Z b_{i}+\epsilon_{i} .
\end{aligned}
$$

The vector $Y_{i}$ gives the fMRI signal measured for subject $i$ and the matrix $Z$ models as in Eq. (1.10) in each column the hemodynamic response to one stimulus type. The fixed effects $\beta=\left(\beta_{1}, \ldots, \beta_{Q}\right)^{T}$ consists of the population amplitudes $\beta_{q}$ to the hemodynamic response of all stimulus types. The parameter vector $\gamma_{i}$ is fixed per subject and represents nuisance parameters with nuisance matrix $S$. The random effects $b_{i}=\left(b_{i 1}, \ldots, b_{i Q}\right)^{T}$ model the between-subject variability and are assumed to be normally distributed with expectation 0 and covariance matrix $D$. The error $\epsilon_{i}$ represents the within-subject variability and 
is normally distributed with expectation 0 and covariance matrix $\Sigma$. It is assumed that there are $N$ subjects and that the matrices $Z, S, \Sigma$ and $D$ are the same for all subjects.

The GLS estimator of $\left(\beta^{T}, \gamma^{T}\right)^{T}=\left(\beta^{T}, \gamma_{1}^{T}, \ldots, \gamma_{N}^{T}\right)^{T}$ is given by

$$
\begin{aligned}
\widehat{\left(\begin{array}{l}
\beta \\
\gamma
\end{array}\right)} & =\left(\sum_{i=1}^{N}[Z S]^{T}\left(Z D Z^{T}+\Sigma\right)^{-1}[Z S]\right)^{-1} \sum_{i=1}^{N}[Z S]^{T}\left(Z D Z^{T}+\Sigma\right)^{-1} Y_{i}, \\
\operatorname{Cov} \widehat{\left(\begin{array}{l}
\beta \\
\gamma
\end{array}\right)} & =\left(\sum_{i=1}^{N}[Z S]^{T}\left(Z D Z^{T}+\Sigma\right)^{-1}[Z S]\right)^{-1} .
\end{aligned}
$$

Since the interest is in the fixed effects $\beta$, we will focus for the optimality criterion on the covariance matrix of $\operatorname{Cov}(\hat{\beta})$. It can be shown that the covariance matrix of $\hat{\beta}$ is given by

$$
\operatorname{Cov}(\hat{\beta})=\frac{1}{N}\left(\left(Z^{T} V^{T}\left(I-P_{V S}\right) V Z\right)^{-1}+D\right) .
$$

$\operatorname{Cov}(\hat{\beta})$ differs from Eq. (1.13) due to the between-subject covariance matrix $D$ and the number of subjects $N$.

\subsubsection{Error structure}

The fMRI signal is temporally correlated (Bullmore et al., 1996; Purdon \& Weisskoff, 1998; Woolrich et al., 2001). Therefore, it is important to account for this temporal correlation in a model to avoid biased hypothesis-testing and biased confidence intervals. In this thesis, we will use three different error structures, that is uncorrelated errors and correlated errors with first-order autoregressive (AR1) error structure (Bullmore et al., 1996) or a first-order autoregressive error plus measurement error (AR1+ME) structure (Purdon \& Weisskoff, 1998). The uncorrelated error structure is not realistic but it is useful to derive analytical results and to compare the results for correlated errors with the results for uncorrelated errors so that the effect of correlated errors can be determined.

The uncorrelated error structure considers homogeneous variance, i.e., $\operatorname{Var}\left(\epsilon_{j}\right)=\sigma_{e}^{2}$ for all time points $j=1, \ldots, T$, and no correlation between errors $\epsilon_{j_{1}}$ and errors $\epsilon_{j_{2}}$ at different time points $j_{1}$ and $j_{2}\left(j_{1}, j_{2}=1, \ldots, T\right)$. A first-order autoregressive error is given by a stationary variance of the error term at each time point and a decreasing correlation between errors at different time points if the time lag between these time points increases. In other words, $\operatorname{Var}\left(\epsilon_{j}\right)=\sigma_{\epsilon}^{2}$ for all $j=1, \ldots, T$ and $\operatorname{Corr}\left(\epsilon_{j_{1}}, \epsilon_{j_{2}}\right)=\rho^{\left|j_{1}-j_{2}\right|}$ $\left(j_{1}, j_{2}=1, \ldots, T\right)$, where the AR1 autocorrelation coefficient $\rho$ is in the interval $(-1,1)$. In the AR1+ME model, the AR1 part represents correlated noise whereas the measurement error represents white (uncorrelated) scanner noise. The variance of the error at a given time point is given by $\operatorname{Var}\left(\epsilon_{j}\right)=\sigma_{\epsilon}^{2}+\sigma_{e}^{2}$ and the correlation is given by $\operatorname{Corr}\left(\epsilon_{j_{1}}, \epsilon_{j_{2}}\right)=$ $\rho^{\left|j_{1}-j_{2}\right|} \sigma_{\epsilon}^{2} /\left(\sigma_{\epsilon}^{2}+\sigma_{e}^{2}\right)$, where $\sigma_{\epsilon}^{2}$ is the variance for the AR1 part and $\sigma_{e}^{2}$ is the variance for the ME part.

\subsection{Optimal design}

Our optimality criteria focus on the covariance matrices of the GLS estimators from the four presented models because the covariance matrix represents a measure of the accuracy 
of the estimator. The unknown parameter vector in our model will be called $\phi$, i.e., $\phi=\left(h^{T}, \gamma^{T}\right)^{T}$ in Model 1, $\phi=\left(\beta^{T}, \gamma^{T}\right)^{T}$ in Model 2, $\phi=\left(\tau^{T}, \gamma^{T}\right)^{T}$ in Model 3 and $\phi=\left(\beta^{T}, \gamma_{1}^{T}, \ldots, \gamma_{N}^{T}\right)^{T}$ in Model 4 . The number of parameters in $\phi$ will be denoted by $p$. To express the dependence of the covariance matrix $\operatorname{Cov}(\hat{\phi})$ of the estimator $\hat{\phi}$ on the $\operatorname{design} \xi$, the covariance matrix $\operatorname{Cov}(\hat{\phi})$ for design $\xi$ will be denoted by $\operatorname{Cov}(\hat{\phi}, \xi)$. In the following, designs will be denoted by $\xi$ and the design space which is the set of all designs will be denoted by $\Xi$.

The considered optimality criteria calculate a single value for each covariance matrix $\operatorname{Cov}(\hat{\phi}, \xi)$ to compare covariance matrices $\operatorname{Cov}(\hat{\phi}, \xi)$ for different designs $\xi$ to each other. By calculation of a single value for a given covariance matrix, an ordering of the covariance matrices is possible. In this thesis we will employ variants of the $A$ - and $D$-optimality criterion as different subsets or contrasts of unknown parameters can be of interest in fMRI data analysis. The $A$-optimality criterion has been applied before in fMRI literature (Dale, 1999; Liu et al., 2001; Wager \& Nichols, 2003). One purpose of this thesis will be to introduce the $D$-optimality criterion to fMRI design optimization and evaluate whether different optimal designs are obtained by the two criteria. The $D$-optimality is a common and popular criterion in other fields of research. Its advantages and disadvantages in comparison with the $A$-optimality criterion will be given in the following Section 1.4.2.

\subsubsection{A-optimality criterion}

The following discussed criteria belong strictly speaking to the $C$-optimality criterion (Atkinson et al., 2007). However, in consistency with fMRI literature the term $A$-optimality criterion is employed in this thesis. The $A$-optimal design is defined as the design that minimizes the trace of the covariance matrix of $\widehat{C \phi}$

$$
\operatorname{trace}(\operatorname{Cov}(\widehat{C \phi}, \xi))=\operatorname{trace}\left(\operatorname{CCov}(\hat{\phi}, \xi) C^{T}\right)
$$

over all designs $\xi$ in the design space $\Xi$. The matrix $C$ has different forms depending on the parameters or parameter combinations of interest. If $C$ equals the identity matrix of order $p$, the $A$-optimality criterion as defined in optimal design literature is obtained and the sum of all variances $\operatorname{Var}\left(\hat{\phi}_{i}, \xi\right)$ is minimized. $\operatorname{Var}\left(\hat{\phi}_{i}, \xi\right)$ gives here the variance of the GLS estimator for parameter $\phi_{i}$ in $\phi$.

Mostly the interest is not in the nuisance parameters but only in the parameters related to the hemodynamic response function, e.g., $h$ in Model 1 (see Eq. (1.2)), or the parameters representing the amplitude, e.g., $\beta$ in Model 2 (see Eq. (1.10)). The number of parameters of interest is denoted by $p_{1}$, e.g., $p_{1}=Q k$, the number of time points for all $Q$ HRFs in Model 1, or $p_{1}=Q$, the number of stimulus effects in Model 2. In this situation a $p_{1} \times p$ matrix $C$ is employed with entries 0 and 1 . This matrix $C=\left[\begin{array}{ll}I_{p_{1}} & 0_{p_{1} \times\left(p-p_{1}\right)}\end{array}\right]$ is a concatenation of the identity matrix $I_{p_{1}}$ of order $p_{1}$ and the $p_{1} \times\left(p-p_{1}\right)$ matrix $0_{p_{1} \times\left(p-p_{1}\right)}$ full of zeros. For such a matrix $C$, we call this criterion the $A_{S^{-}}$or simply the $A$-optimality criterion.

Furthermore, contrasts (linear combinations) $C \phi$ of parameters are often of interest to detect differential activation and localize certain sub-aspects of cognitive processes. The area of face recognition (Fusiform Face Area, FFA) has been detected by contrasting the 
stimulus effect to seeing faces with the stimulus effect to seeing simple objects (Kanwisher et al., 1997). The matrix $C$ is then called the contrast matrix and contains 0's, 1's and -1 's depending on the contrast of interest. If the matrix $C$ is of rank 1 , thus a single vector representing the contrast of interest, the $c$-optimality criterion is obtained.

\subsubsection{D-optimality criterion}

Instead of the trace the determinant can be employed and variants of the $D$-optimality criterion are obtained. The $D$-optimal design is the design that minimizes the determinant of the covariance matrix of the GLS estimator for $C \phi$ :

$$
\operatorname{det}(\operatorname{Cov}(\widehat{C \phi}, \xi))=\operatorname{det}\left(\operatorname{CCov}(\hat{\phi}, \xi) C^{T}\right)
$$

over all designs $\xi$ in the design space $\Xi$. The matrix $C$ is a matrix containing 0 's, 1 's and -1 's as for the $A$-optimality criterion. The value of the $D$-optimality criterion is proportional to the volume of the confidence ellipsoid for $\widehat{C \phi}$. Thus by minimizing $\operatorname{det}(\operatorname{Cov}(\widehat{C \phi}, \xi))$, the $D$-optimal design minimizes the volume of the confidence ellipsoid. A disadvantage of the $D$-optimality is that it is for practitioners more difficult to understand than the $A$-optimality criterion.

For $C=I_{p}$ the actual $D$-optimality criterion as defined in optimal design theory is obtained and the $D$-optimal design is invariant to linear transformation of the design matrix. This means that the same $D$-optimal design as before is obtained after linear transformation of the columns in the design matrix. Such a linear transformation could be due to scaling of the double gamma function to have a maximum value of 1 . As a consequence, the design sub-matrix $Z$ in Model 2 is multiplied with a scaling factor whereas the design sub-matrix $S$ remains unchanged.

With the matrix $C=\left[\begin{array}{ll}I_{p_{1}} & 0_{p_{1} \times\left(p-p_{1}\right)}\end{array}\right], \widehat{C \phi}$ equals $\left(\hat{\phi}_{1}, \ldots, \hat{\phi}_{p_{1}}\right)^{T}$ and the interest is in this subset of parameters. This criterion will be called $D_{S^{-}}$or just $D$-optimality criterion. The $D_{S}$-optimality criterion is also invariant to linear transformation of the design matrix which belongs to the parameters of interest, e.g., the design matrix $Z$ for parameter $\beta$ in Model 2 (see Eq. (1.10)). Furthermore, the matrix $C$ can be a nonsingular matrix formulating the contrasts of interest. This criterion is also called generalized $D$-optimality criterion in optimal design literature but will simply be called $D$-optimality criterion in this thesis.

\subsubsection{Maximin criterion}

Model 1 and Model 2 are considered in Chapter 2 and Chapter 3 for different values of autocorrelation in a first-order autoregressive error (AR1) or for different values of variances and autocorrelation in a first-order autoregressive error plus measurement error (AR1+ME). This means that the covariance matrix $\operatorname{Cov}(\widehat{C \phi}, \xi)$ depends on these parameters for the error structure. The optimal designs for a given variance or autocorrelation are thus only locally optimal, i.e., optimal for the specific error parameters. Furthermore, different values in Model 3 for the hemodynamic response function, i.e., $a_{1}, b_{1}, c, a_{2}$ and $b_{2}$ are considered in Chapter 4 and locally optimal designs for a given parameter set are obtained. 
To handle local optimality, the maximin criterion based on a relative efficiency measure was employed. The maximin criterion chooses a maximin design which minimizes the worst possible case of relative efficiency over a preselected region of parameter values. If the calculated maximin value for the maximin design is high, the maximin design is efficient for all considered parameter values.

The relative efficiency of a design $\xi_{1}$ versus a design $\xi_{2}$ is defined as

$$
\operatorname{RE}\left(\xi_{1} \mid \xi_{2}\right)=\frac{\psi\left(\operatorname{Cov}\left(\widehat{C \phi}, \xi_{2}\right)\right)}{\psi\left(\operatorname{Cov}\left(\widehat{C \phi}, \xi_{1}\right)\right)}
$$

where $\psi$ stands for the optimality criterion with a correction for the number of parameters $s$ in $C \phi$ for the $D$-optimality criterion, i.e., $\psi\left(\operatorname{Cov}\left(\widehat{C \phi}, \xi_{1}\right)\right)=\left[\operatorname{det}\left(\operatorname{Cov}\left(\widehat{C \phi}, \xi_{1}\right)\right)\right]^{1 / s}$ for the $D$-optimality criterion and where $\operatorname{Cov}\left(\widehat{C \phi}, \xi_{1}\right)$ refers to the covariance matrix of the GLS estimator $\widehat{C \phi}$ obtained by design $\xi_{1}$. In this form, the relative efficiency of design $\xi_{1}$ versus design $\xi_{2}$ has the interpretation that it equals the number of times which $\xi_{1}$ has to be repeated to be as efficient as $\xi_{2}$.

The model parameter will be denoted by $\omega$, i.e., parameters of the error $\epsilon$ or parameters of the double gamma function, upon which the locally optimal designs depend. The locally optimal design for a given value of $\omega$ is expressed by $\xi_{\omega}$. The space of all $\omega$ is denoted by $\Omega$. The maximin design is the design that maximizes the smallest relative efficiency:

$$
\operatorname{MMD}=\arg \max _{\xi \in \Xi} \min _{\omega \in \Omega} \operatorname{RE}\left(\xi \mid \xi_{\omega}\right)
$$

In words, for each design $\xi$ the minimum relative efficiency over all parameters $\omega$ in $\Omega$ are obtained in the first step of the maximin procedure. In the second step the design with maximum minimum relative efficiency over all designs $\xi$ in $\Xi$ is considered. The value $\max _{\xi \in \Xi} \min _{\omega \in \Omega} R E\left(\xi \mid \xi_{\omega}\right)$ is called the maximin value (MMV).

\subsection{Outline}

The following Chapters 2-5 in this thesis may be read as self-contained articles. The last chapter (Chapter 6) gives overall conclusions, ideas for future work and a summary of the results in this thesis. The notations and the formulae may slightly vary between chapters to facilitate explanation of a specific problem and increase readability. A brief introduction to the Chapters 2-5 is as follows.

In Chapter 2 the focus is on the optimization of blocked designs. Blocked designs can be characterized by three factors: the length of blocks, i.e., number of trials per blocks, the ordering of task and rest blocks and the time between trials within one block. The optimal combination of these three factors will be determined for three different error structures and different optimality criteria. To handle local optimality the maximin criterion will be applied to find designs which are robust against misspecification of error parameters.

Chapter 3 considers the optimization of blocked and event-related designs. Several design criteria, i.e., detection power and estimation efficiency, are considered as well as a multi-objective criterion based on $A$ - or $D$-optimality. Up to four objectives of design 
optimization, e.g., increase of detection power, increase of estimation efficiency, optimal counterbalancing and maintenance of desired stimulus frequency, are combined into a multiobjective criterion. This chapter provides an extension to the work of Kao et al. (2009) and Liu \& Frank (2004). The genetic algorithm of Kao et al. (2009) is used to determine optimal designs for several autocorrelation values in an AR1 model. The approach of Liu \& Frank (2004) to determine the A-optimal stimulus frequency is adapted to determine the $D$-optimal stimulus frequency. Maximin designs are obtained which are robust against misspecification of the correlation in an AR1 error structure.

In Chapter 4 optimal designs for nonlinear estimation of the HRF are studied. The maximin approach is applied to handle local optimality on the parameters of the double gamma function. The $D$-optimality criterion is used to obtain locally optimal designs. The $A$-optimality criterion was not considered as the invariance property of the $D$-optimality criterion to linear transformation of the design matrix was relevant here. The maximin design chosen from the locally optimal designs of the genetic algorithm is compared to maximin designs from other design types, i.e., blocked designs, rapid event-related designs and slow event-related designs. The rapid event-related designs consists of $m$-sequences and event-related designs with geometric or uniform distribution of ISI. In addition, the optimal design for detection power and estimation efficiency based on a linear model are determined and compared to the maximin design from the genetic algorithm.

Chapter 5 addresses the optimization of blocked multi-subject fMRI experiments. A linear cost function is presented which describes the costs for a multi-subject fMRI design. Blocked designs with $Q$ stimulus types $A_{1}, \ldots, A_{Q}$, block order $A_{1} \ldots A_{Q} N$ and several cycles (repetitions) of $A_{1} \ldots A_{Q} N$ are considered. The $A$-optimality criterion is applied to determine the optimal allocation of number of cycles and number of subjects within a fixed study budget. The $D$-optimality is not considered because it resulted in too complicated analytical results. Furthermore, the $D$-optimality criterion needs specification of values for the off-diagonal elements of the random-effects covariance matrix for estimation of individual stimulus effects and estimation of contrasts between stimulus effects instead of only for contrasts as the $A$-optimality criterion. For uncorrelated errors, analytical expressions for the $A$-optimal number of cycles and subjects are given. For an AR1 error, a method for numerical calculation of the $A$-optimal number of cycles and subjects is given.

Chapter 2, 3 and 4 consider the optimal design sequence given the total duration of an fMRI experiment whereas Chapter 5 considers the optimal duration of a blocked design and the optimal number of subjects given a total study budget. 


\section{CHAPTER 2}

\section{Optimization of blocked designs in fMRI studies}

This chapter is published in Psychometrika (2010), 75(2): 373-390, with co-authors Gerard J. P. van Breukelen, Rainer Goebel and Martijn P. F. Berger. 


\begin{abstract}
Blocked designs in functional magnetic resonance imaging (fMRI) are useful to localize functional brain areas. A blocked design consists of different blocks of trials of the same stimulus type and is characterized by three factors: the length of blocks, i.e., number of trials per blocks, the ordering of task and rest blocks and the time between trials within one block. Optimal design theory was applied to find the optimal combination of these three design factors. Furthermore, different error structures were used within a general linear model for the analysis of fMRI data, and the maximin criterion was applied to find designs which are robust against misspecification of model parameters.
\end{abstract}

Keywords: blocked design, fMRI, optimal design, efficiency, maximin 


\section{$2.1 \quad$ Introduction}

Functional magnetic resonance imaging (fMRI) is widely used in cognitive neuroscience to localize human brain functions (Owen et al., 2001; Chein \& Schneider, 2003). It is also applied to determine the related brain areas to psychiatric disorders, e.g., schizophrenia (Honey \& Bullmore, 2002). Since its invention in the early 1990s, fMRI has led to major advances in understanding the neural mechanisms that underlie cognitive processes, e.g., perception, attention, language, and memory (Chein \& Schneider, 2003). It has established a strong link between cognitive psychology and neuroscientific research. Compared with other neuroimaging methods as, e.g., Positron Emission Tomography (PET), fMRI has advantages in terms of increased spatial resolution (Di Salle et al., 1999). However, one major drawback of fMRI is the low signal-to-noise ratio (SNR). To overcome this weakness, optimal design of fMRI experiments is helpful to increase the signal-to-noise ratio (Josephs \& Henson, 1999).

The fMRI signal in most studies is based on the blood-oxygen-level-dependent (BOLD) contrast which is based on magnetic properties of oxygenated and deoxygenated blood and hemodynamic processes like regional cerebral blood flow (rCBF) caused by neural activity (Logothetis \& Wandell, 2004). The hemodynamic response to one short stimulus can be described by a hemodynamic response function (HRF) (Friston et al., 1994). The double gamma function is mostly employed as HRF and models the temporal evolution of the hemodynamic response over a period of around $30 \mathrm{~s}$ (Friston et al., 1998). There are mainly two different types of design in fMRI experiments: blocked designs and event-related designs (Aguirre \& D'Esposito, 1999; Chein \& Schneider, 2003). In blocked designs, trials of one condition are performed in blocks which can be a task condition or a rest condition, i.e., null block, with no systematic or task-related activation. In contrast, event-related designs can have a random order of trials and a fixed or random time interval between trials (Buckner et al., 1996; Dale \& Buckner, 1997; Rosen et al., 1998).

Blocked and event-related designs are optimal for different purposes, e.g., blocked designs are better for detection of activated brain areas assuming a certain HRF shape whereas event-related designs are useful to estimate the shape of the HRF (Friston et al., 1999; Chein \& Schneider, 2003; Birn et al., 2002; Liu et al., 2001; Liu \& Frank, 2004; Liu, 2004). In the fMRI literature, detection of activation is referred to as detection power, whereas estimation of the HRF is called estimation efficiency. In this paper, the focus is on blocked designs and detection power, but this approach can be applied to event-related designs or estimation efficiency as well. Several authors have generated random event-related designs and determined their estimation efficiency (Buračas \& Boynton, 2002; Hagberg et al., 2001; Wager \& Nichols, 2003; Kao et al., 2009). Maus et al. (2010b) applied the maximin approach to locally optimal event-related designs for estimation efficiency.

The performance of fMRI experiments is very expensive and time consuming. A scanner session for one subject might take one hour which may cost between $\$ 200$ and $\$ 1000$ per hour, not including additional expenses such as subject fees, equipment and staff salaries (Culham, 2006). The number of subjects and repeated measurements may be restricted due to budgetary constraints. Thus, it is necessary to minimize costs and to maximize the efficiency of the estimators. The purpose of this study is to maximize the efficiency within 
a fixed budget.

Some general recommendations for blocked designs are given in the fMRI literature. The most simple design is the alternating boxcar design in Figure 2.1a which consists of alternations between blocks of two conditions. One of these conditions can be a null block where no systematic or task-related stimulation takes place. Thus, the design has the following structure: ANANAN (boxcar design), where A refers to a task condition and $\mathrm{N}$ to a null block, or ABABAB, where $\mathrm{A}$ and $\mathrm{B}$ refer to two different task conditions. Mohamed et al. (2000) showed that an alternating block order ANANAN was more effective than a continuous design AAANNN. Similarly, Nakai et al. (2003) showed that although they used the same number of task blocks $\mathrm{A}$ and $\mathrm{B}$ in each blocked design, detection of functional activity depended on the ordering of task blocks and null blocks, i.e., block order ANBN, $\mathrm{ABN}, \mathrm{AB}$ or order $\mathrm{AN}$ followed by $\mathrm{BN}$.

Recommendations in the literature for optimal block length in an alternating boxcar design range from 14 to 20 seconds (Chein \& Schneider, 2003; Aguirre \& D'Esposito, 1999; Wüstenberg et al., 2005; Skudlarski et al., 1999; Smith et al., 2007). Further, to produce a strong and homogeneous signal, it is optimal for detection to use a short time interval between the presentation of stimuli inside blocks, i.e., short stimulus asynchrony (SOA) (Chein \& Schneider, 2003; Josephs \& Henson, 1999). However, the duration of this interval depends on stimulus duration and the type of stimulus, e.g., a block with a mental rotation task may need a longer time between presentation of trials than a block with trials of a lexical decision task (Chein \& Schneider, 2003).

The approach by Nakai et al. (2003) of fixing the number of task blocks and varying the number of null blocks resulted in different total experimental time periods for each design. In contrast, in our study a fixed time period for the experiment was assumed to determine which design is optimal within a fixed time period and resultant fixed budget. Additional to the block order, the optimization was expanded to other design factors, i.e., stimulus onset asynchrony (SOA) and block length which are displayed in Figure 2.1b. The SOA gives the time between presentation of two stimuli. To our knowledge, there is no study which has considered the optimization of blocked designs considering the influence from several varying design factors simultaneously, i.e., SOA, block length and block order.

This optimization depends on the statistical model for the analysis of fMRI data and the optimality criterion. The general linear model is employed to model fMRI data at one three-dimensional imaging unit (Friston et al., 1995). Several approaches to model the error have been undertaken, such as an uncorrelated error structure, which is the most simple case. However, it is known that fMRI data and the noise in fMRI data are temporally correlated and can thus be better fitted by a linear model with correlated error, notably autoregressive error of order 1 (AR1) (Bullmore et al., 1996; Worsley, 2005; Chatfield, 2004) and AR1 plus measurement error (AR1+ME) (Purdon \& Weisskoff, 1998). Ignoring autocorrelation will bias hypothesis-testing and confidence interval estimation as the error covariance matrix and the degrees of freedom of test statistics are incorrectly estimated (Worsley \& Friston, 1995; Bullmore et al., 1996; Purdon \& Weisskoff, 1998). According to Gautama \& Van Hulle (2005) AR1 is the most frequently applied.

In the literature several optimality criteria were proposed which are all functions of 


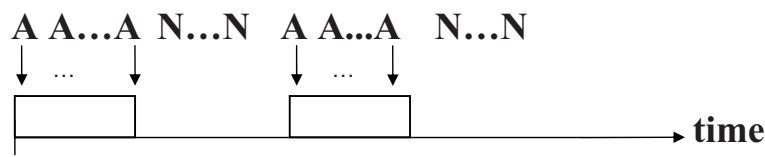

(a) Illustration of alternating boxcar design, A denotes task (stimulus) block and $\mathrm{N}$ a null (rest) block.

\section{Block order ANBN (one alternation)}

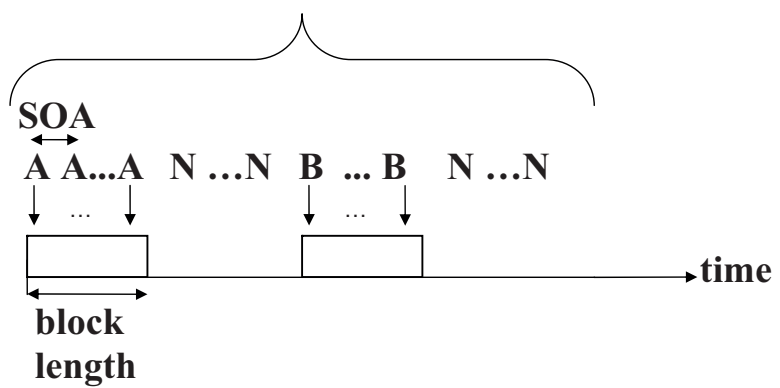

(b) Illustration of design factors, $\mathrm{A}$ and $\mathrm{B}$ denote task (stimulus) blocks whereas $\mathrm{N}$ denotes null (rest) block.

Figure 2.1: Blocked designs

the covariance matrix of the parameter estimators in the linear model. In this paper, the determinant or $D$-optimality criterion, the trace or $A$-optimality criterion and the contrast or c-optimality criterion (Atkinson \& Donev, 1996, chap. 4, 10; Silvey, 1980) were used, and these will be explained in Section 2.3.1. As far as we know, the $D$-optimality criterion has not been considered before for optimization of fMRI experiments. Methods from signal processing like power spectrum analysis have been applied to determine the optimal block length (Aguirre \& D'Esposito, 1999), whereas the $A$-optimality criterion has mostly been applied for optimization of event-related designs (Dale, 1999; Friston et al., 1999; Liu et al., 2001; Liu \& Frank, 2004; Wager \& Nichols, 2003; Kao et al., 2009). Friston et al. (1999) have also used the $c$-optimality criterion to optimize detection power for contrasts between stimulus effects.

However, $A-, D-$, and $c$-optimal designs depend on the model parameters, e.g., the autocorrelation in an AR1 error structure, and are thus only locally optimal. This problem will be solved by using the maximin criterion based on a relative efficiency measure. The maximin approach has been applied in optimal design theory to construct designs which are robust against misspecification of parameters in various models, e.g., the linear mixed model (Berger \& Tan, 2004; Ouwens et al., 2002) or exponential regression models (Dette et al., 2006). The effect of autocorrelation on optimal design has been studied before in the fMRI literature by assuming fixed parameter values in the error structure or by using real fMRI noise (Aguirre \& D'Esposito, 1999; Smith et al., 2007; Wager \& Nichols, 2003; Zarahn \& Friston, 2002; Buračas \& Boynton, 2002; Burock \& Dale, 2000). As a result, the found optimal designs may not be robust against misspecification of these parameters.

To summarize, the objective of this paper is to determine the optimal blocked design for fMRI studies with respect to detection power. Three design factors, namely block 
length, ordering of blocks and time between trials within one block, will be considered simultaneously to find the optimal combination of these factors. The time period of the experiment is assumed to be fixed. Furthermore, the maximin criterion is applied to solve the problem of local optimality due to a temporally correlated noise structure.

The outline of this paper is as follows. In Section 2.2.1 the general linear model is presented. For numerical calculations, a specific hemodynamic response function was chosen, which is introduced in Section 2.2.1. The different covariance matrices according to the different error structures are explained in Section 2.2.2. The results of our analysis of a blocked experiment are presented in Section 2.2.3, confirming the assumptions made in Section 2.2.1. The different optimality criteria, the maximin criterion and the chosen values for the design factors SOA, block length and block order are presented in Section 2.3. The results are described in Section 2.4 separately for the different error structures and optimality criteria. Finally, in Section 2.5 results are discussed, and conclusions are presented.

\section{$2.2 \quad$ Model}

\subsubsection{General linear model}

Considering an experiment with two stimulus types $\mathrm{A}$ and $\mathrm{B}$, the fMRI signal $Y$ at one voxel of one subject can be described by a general linear model (GLM). A voxel-wise analysis is then performed, where one voxel corresponds to one three-dimensional imaging unit, for instance, $3 \mathrm{~mm} \times 3 \mathrm{~mm} \times 3 \mathrm{~mm}$, in the subject's brain. The model is of the form

$$
Y_{i}=X_{i 1} \beta_{0}+X_{i 2} \beta_{1}+X_{i 3} \beta_{2}+X_{i 4} \beta_{3}+\varepsilon_{i}
$$

where $Y_{i}$ refers to the signal at time point $i(i=1, \ldots, n)$, and $n$ is the number of time points of the experiment. By the model in Eq. (2.1), one fMRI run which is one continuously scanned time period can be described. The signal is normally sampled within the range of 5-10 min to avoid subject's fatigue and with a repetition time (TR) of several seconds, e.g., 2-4 s, to guarantee full brain coverage (Henson, 2004). The unknown parameters are the intercept $\beta_{0}$, effects $\beta_{1}$ and $\beta_{2}$ of stimuli $\mathrm{A}$ and $\mathrm{B}$, and an effect $\beta_{3}$ for linear trend. The main interest is in the stimulus effects $\beta_{1}$ and $\beta_{2}$. In addition, a random error $\varepsilon_{i}$ is present. The expected hemodynamic responses at time point $i$ are reflected by $X_{i 2}$ and $X_{i 3}$, the linear trend is modeled by regressor $X_{i 4}=(i-1) \cdot \mathrm{TR}+1 \forall i$ and the fMRI baseline signal by $X_{i 1}=1 \forall i$.

To calculate the hemodynamic response, a specific hemodynamic response function has to be assumed. In this paper, the sum of two gamma functions was applied (Friston et al., 1998):

$$
\mathrm{h}(t)=\frac{b_{1}^{a_{1}+1} \cdot t^{a_{1}} \cdot \exp \left(-b_{1} t\right)}{\Gamma\left(a_{1}+1\right)}-\frac{b_{2}^{a_{2}+1} \cdot t^{a_{2}} \cdot \exp \left(-b_{2} t\right)}{c \cdot \Gamma\left(a_{2}+1\right)}
$$

with parameters $a_{1}, b_{1}, c, a_{2}$ and $b_{2}$, where $a_{1} / b_{1}$ gives the time to the maximum for the positive gamma function (time to peak), and $a_{2} / b_{2}$ gives the time to the minimum for the negative gamma function (time to undershoot). Parameter $c$ influences the ratio of the 
amplitudes of the positive and negative gamma function (peak-to-undershoot amplitude ratio). The first gamma function, known as the positive gamma function, models the peak of the hemodynamic response, while the second gamma function, the negative gamma function, models an undershoot. The HRF was parameterized by $a_{1}=5, b_{1}=1, c=$ $6, a_{2}=15$ and $b_{2}=1$ that are appropriate for a brief stimulus (Henson, 2004), e.g., 1 s. The resulting HRF can be seen in Figure 2.2a. Additionally, it is common to assume that the hemodynamic response acts as the output of a linear time-invariant system (LTI) (Boynton et al., 1996; Cohen, 1997; Dale \& Buckner, 1997; Henson, 2004). Linearity means that the property of superposition is fulfilled so that the hemodynamic responses to successive stimuli are summed up to the final hemodynamic response as illustrated in Figure 2.2b. Invariance implies that the form of the hemodynamic response is independent of time (Henson, 2004).

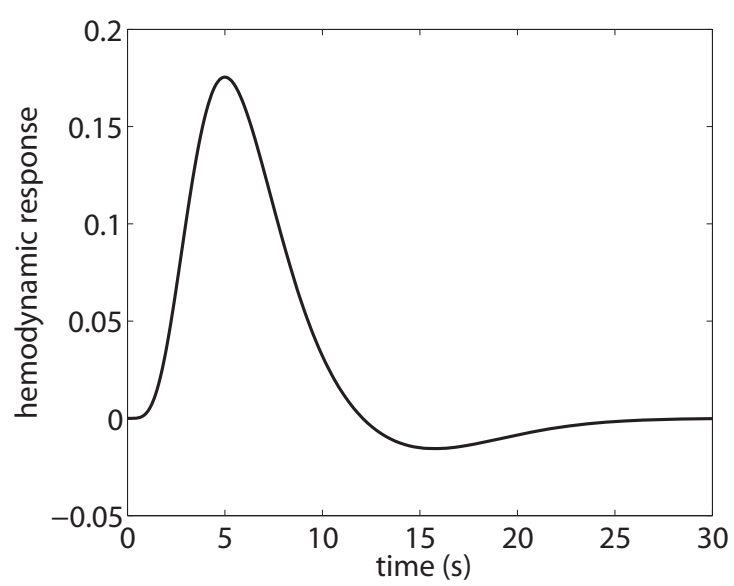

(a) Hemodynamic response function as sum of two gamma functions after presentation of stimulus $\mathrm{A}$ at $0 \mathrm{~s}$

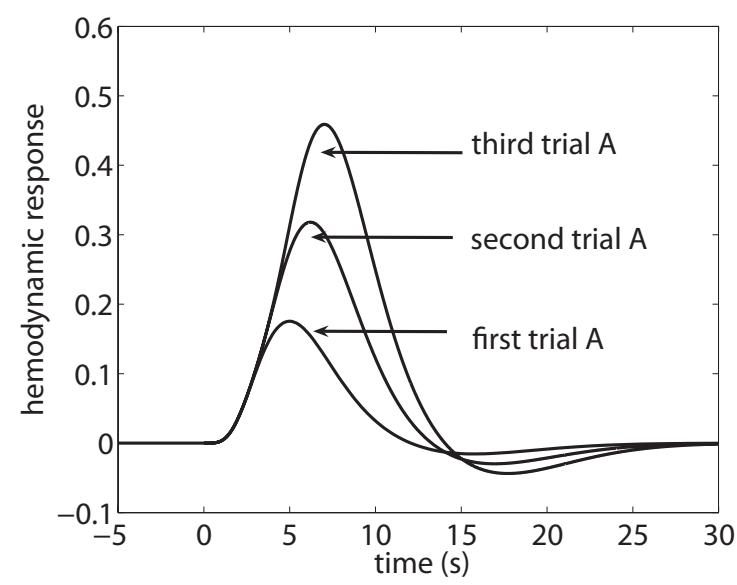

(b) Linearity of hemodynamic response after presentation of stimulus A at $0 \mathrm{~s}, 2 \mathrm{~s}$ and $3 \mathrm{~s}$

Figure 2.2: Hemodynamic response

Assuming linearity, invariance and a specific HRF, the expected hemodynamic response $X_{i j}$ at time point $i$ to stimulus A, resp. B, can then be described mathematically by convolution to model the enduring hemodynamic response function after stimulus presentation and the superposition of hemodynamic responses to successive stimuli. The following equation gives the convolution $\mathbf{s} \otimes \mathbf{h}$ of a stimulus vector $\mathbf{s}_{\mathrm{A}}$, resp. $\mathbf{s}_{\mathrm{B}}$, with the HRF vector $\mathbf{h}$ at time point $i$ :

$$
\begin{aligned}
& X_{i 2}=\left(s_{\mathrm{A}} \otimes \mathbf{h}\right)(i)=\sum_{l=1}^{k+1} s_{\mathrm{A}}(i-l+1) \mathbf{h}(l) \\
& X_{i 3}=\left(s_{\mathrm{B}} \otimes \mathbf{h}\right)(i)=\sum_{l=1}^{k+1} s_{\mathrm{B}}(i-l+1) \mathbf{h}(l)
\end{aligned}
$$

The vectors $\mathbf{s}_{\mathbf{A}}$ and $\mathbf{s}_{\mathbf{B}} \in \mathbb{R}^{n}$ have entries 0 and 1 , where 0 indicates no stimulus presentation of $\mathrm{A}$, resp. $\mathrm{B}$, at time point $i$, and 1 indicates a stimulus presentation of $\mathrm{A}$, resp. $\mathrm{B}$, at time point $i, i=1, \ldots, n$. The HRF vector $\mathbf{h}$ represents the hemodynamic response function 
sampled at $0,1 \mathrm{TR}, 2 \mathrm{TR}, \ldots, k \mathrm{TR}$, where $k+1$ is the assumed number of time points for the hemodynamic response function.

In matrix notation, the general linear model in Eq. (2.1) can be written as

$$
Y=\mathrm{X} \beta+\varepsilon
$$

The vector $Y=\left(Y_{1}, \ldots, Y_{n}\right)^{T}$ is the fMRI signal, while $\beta$ is the vector of the parameters $\beta_{0}, \beta_{1}, \beta_{2}$ and $\beta_{3}$. The design matrix $\mathrm{X}$ is an $n \times 4$ matrix and contains the entries $X_{i j}$ in the $j$ th column. The errors $\varepsilon$ are assumed to be normally distributed with covariance matrix $\operatorname{Cov}(\varepsilon)$.

The generalized least squares (GLS) estimator of $\beta$ is given by

$$
\hat{\beta}=\left[\mathrm{X}^{T} \operatorname{Cov}(Y)^{-1} \mathrm{X}\right]^{-1} \mathrm{X}^{T} \operatorname{Cov}(Y)^{-1} Y .
$$

The GLS estimator is the best linear unbiased estimator. Assuming the errors to be normally distributed, it is also the maximum likelihood estimator. The $4 \times 4$ covariance matrix of $\hat{\beta}$ is equal to

$$
\operatorname{Cov}(\hat{\beta})=\left[\mathrm{X}^{T} \operatorname{Cov}(Y)^{-1} \mathrm{X}\right]^{-1}
$$

\subsubsection{Covariance structure}

Three different covariance structures for the errors in Eq. (2.1) were considered, always assuming the error to be normally distributed. Firstly, the error in the voxel-wise GLM was assumed to follow an AR1 structure (Chatfield, 2004):

$$
\varepsilon_{1, i}=\rho \varepsilon_{1, i-1}+\delta_{1, i} \forall i \in\{1, \ldots, n\},
$$

where $\rho \in(-1,1)$ and $\delta_{1, i} \sim N\left(0, \sigma_{1}^{2}\right) \forall i$ are independent and identically distributed, i.e., uncorrelated noise. The AR1 model involves stationarity, which means that $E\left(\varepsilon_{1, i}\right)=0$ and $\operatorname{Var}\left(\varepsilon_{1, i}\right)=\sigma^{2} \forall i \in\{1, \ldots, n\}$. Another property of AR1 is that the covariance of the errors decreases with their separation in time: $\operatorname{Cov}\left(\varepsilon_{1, i}, \varepsilon_{1, j}\right)=\rho^{|i-j|} \cdot \sigma^{2}$, where $|\rho|<1$ and $i, j=1, \ldots, n$. The symbol $\rho$ denotes the autocorrelation parameter.

Secondly, AR1+ME was considered for the error structure and thirdly, uncorrelated error (UE). The AR1+ME model is described by the equation

$$
\varepsilon_{2, i}=\varepsilon_{1, i}+\delta_{2, i} \forall i \in\{1, \ldots n\},
$$

where $\varepsilon_{1, i}$ is defined as in Eq. (2.8), and $\delta_{2, i} \forall i$ are normally distributed with expectation 0 and variance $\sigma_{2}^{2}$ and are independent and identically distributed. AR1+ME is more general than AR1, whereas UE is a special case of AR1 and AR1+ME. In the fMRI literature, AR1+ME is often referred to as AR1 plus white noise (Purdon \& Weisskoff, 1998). The AR1 term in Eq. (2.8) is supposed to model the correlated structure of fMRI noise, whereas the measurement error $\delta_{2}$ models scanner noise.

The covariance matrix $\operatorname{Cov}\left(\varepsilon_{2}\right)$ for AR1+ME consists of the covariance matrix $\sigma^{2} \mathrm{~V}$ for the AR1 term plus a covariance matrix for the measurement error $\delta_{2}$. Thus, the covariance matrix of $\varepsilon_{2}$ is given by $\operatorname{Cov}\left(\varepsilon_{2}\right)=\sigma^{2} \mathrm{~V}+\sigma_{2}^{2} \mathrm{I}_{n}$, where $V_{i j}=\rho^{|i-j|}$ for $i, j \in\{1, \ldots, n\}$ and $\mathrm{I}_{n}$ is the identity matrix of dimension $n$. The correlation between two errors is then given by $\operatorname{Corr}\left(\varepsilon_{2, i}, \varepsilon_{2, j}\right)=\sigma^{2} \rho^{|i-j|} /\left(\sigma^{2}+\sigma_{2}^{2}\right)$. 


\subsubsection{Checking of assumptions}

Assumptions about the specific form of the HRF and the linearity of the HRF were made in Section 2.2.1. It is known that there are problems of linearity for rapidly following trials. Dale \& Buckner (1997) showed that for an SOA equal to $2 \mathrm{~s}$, hemodynamic responses of stimuli add in a roughly linear way, but subtle deviations from linearity were obtained. One result of nonlinearity is a decrease in the amplitude, i.e., the hemodynamic response received by assumption of linearity is higher than the real hemodynamic response. This effect is called saturation (Henson, 2004). Furthermore, the shape of the hemodynamic response is slightly changed (Dale \& Buckner, 1997).

While nonlinearity is a complex issue of which the causes and behavior are not completely understood, the reduction of amplitude for following trials in a sequence of events has been consistent in studies (Pollmann et al., 1998; Huettel \& McCarthy, 2000; Miezin et al., 2000; Wager et al., 2005). We decided to consider the effect of amplitude reduction by scaling the amplitude of the HRF. This is a simple approach to nonlinearity, which has already been applied by Wager \& Nichols (2003) and Heckman et al. (2007).

Data from a blocked experiment were used to check our assumptions about the hemodynamic response and the AR1 model. The fMRI data were acquired with a gradient-echo EPI sequence $\left(\mathrm{TE}=60 \mathrm{~ms}, \mathrm{FA}=90^{\circ}, \mathrm{TR}=2000 \mathrm{~ms}, \mathrm{FOV}=200 \times 200 \mathrm{~mm}\right.$, voxel size $=$ $1.6 \times 1.6 \times 3 \mathrm{~mm}, 250$ scans) and are an extended version of the experiment described in Formisano et al. (2004). Their experiment had two experimental conditions (left and right hemifield visual stimulation) alternated with fixation blocks, while our data were derived from an experiment with three experimental conditions (left, right and both hemifield visual stimulation) alternated with fixation blocks.

Colored images of natural objects were presented to one healthy subject for $1 \mathrm{~s}$ within a stimulation block and directly replaced by the next image without an interstimulus interval (Formisano et al., 2004). Further details on experimental procedures and materials can be found in Formisano et al. (2004) and Goebel et al. (2001). The task blocks were of length $36 \mathrm{~s}$, whereas the fixations blocks were of length $14 \mathrm{~s}$ or $18 \mathrm{~s}$. Preprocessing was performed using Brain Voyager QX (Brain Innovation, Maastricht, The Netherlands, www.brainvoyager.com) and included 3D motion correction, slice-time correction and a highpass filter (including linear trend removal) in the time domain. Finally, the data were transformed to the Talairach space. The averaged time course from a region of interest (ROI) consisting of a volume of $773 \mathrm{~mm}^{3}$ was analyzed.

The assumption about the error structure was checked on the residuals of this time series after fitting a general linear model with uncorrelated errors. The residuals could be better described by an AR1 structure than by uncorrelated error or moving average (MA). Furthermore, the assumption of the specific form of the HRF was controlled by assuming different HRF models from the literature (Cohen, 1997; Boynton et al., 1996) instead of the double gamma function. We fitted the data from the blocked experiment by a general linear model based on the double gamma function in Eq. (2.2) with $a_{1}=5, b_{1}=1, c=$ $6, a_{2}=15$, and $b_{2}=1$ and by general linear models based on single gamma functions (Boynton et al., 1996; Cohen, 1997). The single gamma function of Boynton et al. (1996) was parameterized by $n=3, \delta=2.5$, and $\tau=1.25$, and the single gamma function of 
Cohen was parameterized by $k=1$. The double gamma function fitted the data slightly better with an R-square equal to 0.70, whereas the single gamma functions as applied by Boynton et al. (1996) or Cohen (1997) resulted in an R-square of 0.65 or 0.67.

Furthermore, different parameter values for the double gamma function were applied to check for effects of nonlinearity on the HRF shape in our data. However, the R-square values showed that the canonical parameter set described the HRF best. Figure 2.3 shows the observed time course in the region of interest and the predicted time course from our general linear model based on a double gamma function and AR1 error structure. It can be seen that a good fit to the observed time course is given by our predicted time course. The double gamma HRF with AR1 noise was therefore used in the sequel for design optimization.

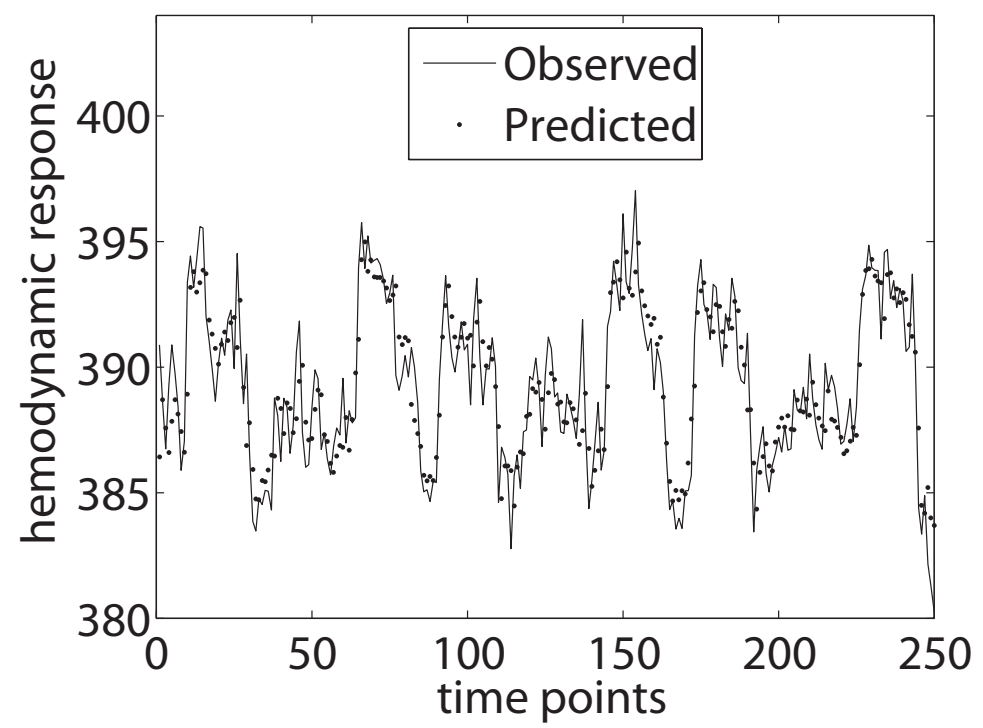

Figure 2.3: Observed time course from region of interest of blocked experiment described in Section 2.2.3 and predicted time course from the general linear model based on double gamma function and AR1 error structure

\subsection{Methods}

Computations were performed with MATLAB (The MathWorks Inc., Natick, MA) and the default double gamma function from the MATLAB toolbox BVQXtools_v07b (Brain Innovation, Maastricht, The Netherlands) was applied. The MATLAB code is available upon request from the first author. Measurements of $Y_{i}$ were supposed to be taken with a sampling rate, repetition time (TR), of 2 seconds. Covariance matrices $\operatorname{Cov}(\hat{\beta})$ were obtained by numerical computations using Eq. (2.7) based on the values for the error parameters described in Section 2.3.3 and the values for the design factors given in Section 2.3.4. The covariance matrices are independent of a specific value of the $\beta^{\prime} s$ as Eq. (2.7) shows. 


\subsubsection{Optimality criteria}

Considering general linear models, each design $\xi$ in the design space $\Xi$ results in a specific design matrix $\mathrm{X}_{\xi}$. In our case, the design space $\Xi$ was finite and consisted of blocked designs characterized by the factors SOA, block length and block order. We will denote by $\hat{\beta}_{\xi}$ the estimator of $\beta$ in Eq. (2.6) which was obtained by the design matrix $\mathrm{X}_{\xi}$ of design $\xi$.

The $D$-optimal design minimizes the determinant of the covariance matrix $\operatorname{Cov}(\hat{\beta})$ (Atkinson \& Donev, 1996, chap. 10)

$$
\operatorname{det}\left[\operatorname{Cov}\left(\hat{\beta}_{\xi}\right)\right]=\operatorname{det}\left\{\left[\mathrm{X}_{\xi}^{T} \operatorname{Cov}(\varepsilon)^{-1} \mathrm{X}_{\xi}\right]^{-1}\right\}
$$

over all designs $\xi$ in $\Xi$. One important property of the $D$-optimal design is that it results in minimizing the volume of the confidence ellipsoid of the parameters. Furthermore, the optimal design remains unchanged for different scaling of the variables in the design matrix $\mathrm{X}$. The $D$-optimality criterion is frequently applied in many research areas because of these two useful properties.

If one is only interested in the variances of estimators, the $A$-optimality criterion may be used, which focuses on the trace of the covariance matrix $\operatorname{Cov}(\hat{\beta})$ (Atkinson \& Donev, 1996, chap. 10). A design is called $A$-optimal if it minimizes

$$
\operatorname{trace}\left[\operatorname{Cov}\left(\hat{\beta}_{\xi}\right)\right]=\operatorname{trace}\left\{\left[\mathrm{X}_{\xi}^{T} \operatorname{Cov}(\varepsilon)^{-1} \mathrm{X}_{\xi}\right]^{-1}\right\}
$$

over all designs $\xi$ in $\Xi$. So, the $A$-optimal design minimizes the average variance of the parameter estimators.

The baseline effect $\beta_{0}$ and effect of linear trend $\beta_{3}$ are in general of no interest in fMRI research. We therefore use a special case of the $L$-optimality criterion (Atkinson \& Donev, 1996, chap. 10), which we will call the $A_{S}$-optimality criterion, and the $D_{S^{-}}$optimality criterion to concentrate on a subset $S$ of parameters (Atkinson \& Donev, 1996, chap. 10) $\beta^{S}=\left(\beta_{1}, \beta_{2}\right)^{T}$, which represents the stimulus effects of interest. The $A_{S^{-o p t i m a l}}$ design

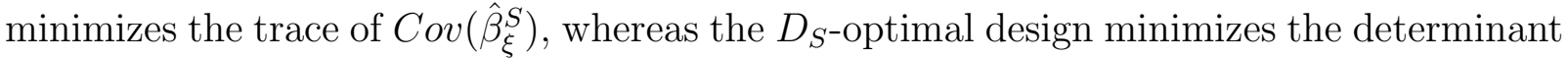
of $\operatorname{Cov}\left(\hat{\beta}_{\xi}^{S}\right)$ for all designs $\xi$ in $\Xi$.

By subtraction of effect parameters for two stimuli it can be determined which brain region is involved in a certain process. The area of seeing faces (Fusiform Face Area, FFA) is, e.g., detected by comparing the hemodynamic response to faces with the response to simple objects. Optimizing then means minimizing the variance of a parameter contrast and leads to a $c$-optimal design. The $c$-optimal design minimizes

$$
\operatorname{Var}\left(c^{T} \hat{\beta}_{\xi}\right)=c^{T} \operatorname{Cov}\left(\hat{\beta}_{\xi}\right) c
$$

for a given contrast vector $c^{T} \in \mathbb{R}^{4}$ with $\sum_{i=1}^{4} c_{i}=0$ over all designs $\xi$ in the design space $\Xi$. Here, the difference $\beta_{1}-\beta_{2}$ given by the contrast vector $c^{T}=(0,1,-1,0)$ is of interest. To summarize, five different optimal design criteria are applied: the $D_{-}, D_{S^{-}}, A^{-}, A_{S^{-}}$and c-optimality criteria.

The relative efficiency (RE) can be used to compare a design $\xi$ with the optimal design $\xi^{*}$. If we denote our optimality criterion by $\psi$, the relative efficiency of $\xi$ versus $\xi^{*}$ is defined as

$$
\operatorname{RE}\left(\xi \mid \xi^{*}\right)=\frac{\psi\left(\operatorname{Cov}\left(\hat{\beta}_{\xi^{*}}\right)\right)}{\psi\left(\operatorname{Cov}\left(\hat{\beta}_{\xi}\right)\right)} .
$$


For the $D$ - and $D_{S}$-optimality criteria, the $p$ th root has to be taken, where $p$ equals the number of parameters, i.e., $p=4$ for $\beta$ and $p=2$ for $\beta^{S}$. By this, the relative efficiency has the same interpretation for all optimality criteria, namely, that the inverse of the RE gives the number of times the design $\xi$ has to be replicated to be as efficient as the optimal design. The relative efficiency of an arbitrary design versus the optimal design lies between 0 and 1 , with a relative efficiency close to 1 being most desirable.

\subsubsection{Maximin approach}

The covariance matrix $\operatorname{Cov}(\hat{\beta})$ depends on the parameters of the error structure. Therefore, the optimal design depends on $\rho$ in the case of AR1 or on $\rho$ and $\sigma^{2} / \sigma_{2}^{2}$ in the case of AR1+ME. This is called local optimality. There are several approaches to handle local optimality, e.g., the sequential approach (Silvey, 1980), the Bayesian design approach (Chaloner \& Verdinelli, 1995; Atkinson \& Donev, 1996, chap. 19) and the maximin approach (Ouwens et al., 2002; Silvey, 1980, p. 59). Due to the high costs related to fMRI experiments, the sequential approach which needs several performances of experiments is not advantageous (Flaherty et al., 2006). The Bayesian approach requires assumptions about a prior distribution of the unknown parameters. For fMRI experiments, such a prior distribution is, however, not generally known for the parameters in the error structure. One disadvantage of the Bayesian approach is furthermore that the chosen optimal design may be efficient for the most likely parameter values but inefficient for a certain parameter value (Pronzato \& Walter, 1988). Then the optimal design is not robust against misspecification of the parameter values.

The maximin approach maximizes the smallest relative efficiency and leads thereby to designs which are robust against misspecification of parameters if the maximum relative efficiency is high. We are searching for the maximin design (MMD) which fulfills the following condition in case of AR1 and for $\rho$ in the parameter space $P_{1} \subseteq[0,1)$ :

$$
\mathrm{MMD}=\arg \max _{\mathrm{SOA}, \mathrm{BL}, \mathrm{BO}} \min _{\rho \in P_{1}} \operatorname{RE}\left(\xi \mid \xi^{*}\right) .
$$

Firstly, given a certain optimality criterion and $\rho$, the optimal design $\xi^{*}$ is determined. Secondly, for each design $\xi$ with certain SOA, block length BL and block order BO the minimum relative efficiency $(\mathrm{RE})$ is determined over the possible autocorrelation parameter values $\rho$ in $P_{1}$. Thirdly, out of these minimum RE values the design with the largest value is chosen to be the maximin design for the optimality criterion. For AR $1+\mathrm{ME}$ and $\rho \times\left(\sigma^{2} / \sigma_{2}^{2}\right)$ in $P_{1} \times P_{2} \subseteq[0,1) \times[0,+\infty)$, the condition is

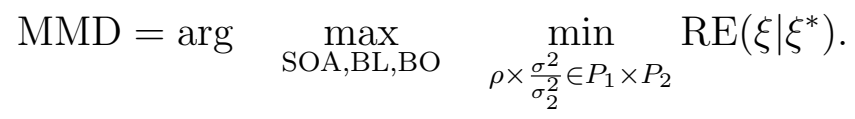

The maximum value in Eqs. (2.14) and (2.15) is called the maximin value (MMV).

\subsubsection{Search for locally optimal design}

For AR1, the autocorrelation parameter $\rho$ was varied by steps of 0.001 to detect optimal designs for a given $\rho$. We assumed $\rho$ to lie in the interval $[0,0.5]$, as this range includes 
common values of correlation for two successive measurements $Y_{i}$ and $Y_{i+1}$, respectively $\varepsilon_{i}$ and $\varepsilon_{i+1}$, in fMRI data with $\mathrm{TR}=2 \mathrm{~s}$. For the AR1 model, $\rho$ is the correlation of two successive data points, whereas for AR $1+\mathrm{ME}$, the correlation is $\rho \sigma^{2} /\left(\sigma^{2}+\sigma_{2}^{2}\right)$.

For AR1+ME, the ratio $\sigma^{2} / \sigma_{2}^{2}$ was considered to be $0.25,0.5,1,2$ and 4 . In order to have a correlation of 0 to 0.5 between two adjacent measurements just as for AR 1 , we chose the autocorrelation parameter $\rho$ to be in the interval $[0,0.7]$. This results in a correlation of maximally 0.56 for all ratios of the variances. Lower values of the ratio will lead to an approximation of AR1+ME to the uncorrelated error structure, whereas higher values of the ratio will resemble the AR1 structure. The autocorrelation parameter was varied by steps of 0.01 to find an optimal design for a given $\rho$ and given ratio $\sigma^{2} / \sigma_{2}^{2}$.

\subsubsection{Design factors}

We chose to fix the stimulus duration at $1 \mathrm{~s}$, thus not letting it play a role in our optimization. It is reasonable to choose an SOA close to the stimulus duration because this will result in a strong fMRI signal and no time between trials is wasted. Therefore, SOAs of 1 , 2 and 3 seconds were applied.

Block length was the time from the first to the last trial in this block plus SOA. As block lengths usually lie between 10 s to 1 min (Chein \& Schneider, 2003; Donaldson \& Buckner, 2001), we covered this range by using 10, 15, 20, 30 and $60 \mathrm{~s}$ of block lengths. To test for the effect of the null blocks, we chose the following block orders: AB, ABN and ANBN. The order of blocks is fixed for one design but repeated several times within the given time interval. For example, block order $\mathrm{AB}$ describes an experiment with repetition of $\mathrm{AB}$ (ABABABAB ... ) until the experiment is finished. In many studies it is necessary to use an active control condition instead of a resting condition. The null condition refers here to simple control conditions with no systematic or task-related activation, e.g., a fixation task or passive visual task, while more complex control conditions are considered as another task/stimulus type.

As time interval for the full modeled experiment, $720 \mathrm{~s}$ were needed because within this period, the block orders $\mathrm{ANBN}, \mathrm{AB}$, and $\mathrm{ABN}$ can be applied with several alternations and blocks of maximally $60 \mathrm{~s}$. To cover the hemodynamic response to the last trial, the time period was finally expanded to $750 \mathrm{~s}$ for all designs resulting in 375 time points because TR was $2 \mathrm{~s}$.

\subsection{Results}

\subsubsection{Autoregressive error of order 1}

\subsubsection{Maximin designs and optimal designs}

The maximin designs for all optimality criteria had block length equal to $15 \mathrm{~s}$ and an SOA of $1 \mathrm{~s}$. For the $D^{-}, D_{S^{-}}$and $A_{S^{-}}$optimality criteria, the maximin design had block order ABN, for the $A$-optimality criterion, the maximin block order was ANBN and for the $c$ optimality criterion, the block order was AB. The locally optimal designs had block lengths 
of $10 \mathrm{~s}, 15 \mathrm{~s}$ or $20 \mathrm{~s}$ for some subintervals of $[0,0.5]$ for $\rho$. Block length had a small effect on the minimum relative efficiency in comparison with block order and SOA. The effect of SOA was stronger than might be expected. As a result, for all optimality criteria except the $D$-optimality criterion, a design with $\mathrm{SOA}=2 \mathrm{~s}$ resulted in only $25 \%-33 \%$ of the minimum relative efficiency of a design with $\mathrm{SOA}=1 \mathrm{~s}$, if block length and block order were kept constant. $\mathrm{SOA}=3 \mathrm{~s}$ resulted in $11 \%-16 \%$ of the minimum relative efficiency of $\mathrm{SOA}=1 \mathrm{~s}$ for all optimality criteria except the $D$-optimality criterion. For the $D$-optimality criterion, these values were $50 \%$ comparing an SOA of $2 \mathrm{~s}$ with an SOA of $1 \mathrm{~s}$ and $34 \%$ comparing 3 $\mathrm{s}$ with $1 \mathrm{~s}$. The separate results from each optimality criterion are further described below and can be seen in Figure 2.4, which shows the minimum relative efficiencies for all designs and optimality criteria. Table 2.1 gives an overview of the maximin designs.

Since centering time $X_{i 4}$ in Eq. (2.1) affects $\beta_{0}$ and thereby potentially may affect the $A$-optimal design, we also applied a centered linear trend, where $X_{i 4}=(i-1) \cdot \mathrm{TR}-374 \forall i$ instead of $X_{i 4}=(i-1) \cdot \mathrm{TR}+1 \forall i$ as for the following results. The variance of the intercept estimator $\operatorname{Var}\left(\hat{\beta}_{0}\right)$ was smaller for the centered time trend, but this affected neither the locally optimal design nor the maximin design. For all optimality criteria, the locally optimal design and maximin design remained the same as for the uncentered time trend.

$D$ - and $D_{S}$-optimality criteria. The minimum relative efficiencies for the $D$-optimality criterion are presented in Figure 2.4a. The maximin value was equal to 0.97. Locally optimal were the designs with block length $10 \mathrm{~s}$ or $15 \mathrm{~s}, \mathrm{SOA}=1 \mathrm{~s}$, and block order $\mathrm{ABN}$, although there was not much difference between $\mathrm{ABN}$ and $\mathrm{ANBN}$. For all other block lengths than the optimal value $15 \mathrm{~s}$, minimum relative efficiencies were slightly lower.

The results for the $D_{S^{-}}$optimality criterion were similar to those for the $D$-optimality criterion, as can be seen in Figures $2.4 \mathrm{a}$ and $2.4 \mathrm{~b}$. For the $D_{S^{-}}$optimality criterion, the maximin value was equal to 0.94 . The only difference seemed to be in the height of the minimum relative efficiencies but not in the order of the various lines, i.e., designs. The same locally optimal designs and the same maximin design as for the $D$-optimality criterion were obtained.

$A$ - and $A_{S}$-optimality criteria. Figure $2.4 \mathrm{c}$ shows the minimum relative efficiencies for the $A$-optimality criterion. $A$-optimal were the designs with $\mathrm{SOA}=1 \mathrm{~s}, \mathrm{BL}=10 \mathrm{~s}, 15 \mathrm{~s}$ or $20 \mathrm{~s}$ and block order ANBN. The design with $\mathrm{SOA}=1 \mathrm{~s}$, block length $15 \mathrm{~s}$ and block order ANBN was the maximin design. The $A_{S}$-optimality criterion gave similar results as the $A$-optimality criterion, except that the locally optimal designs and maximin design had block order ABN instead of ANBN, see Figure 2.4d. The maximin values were 0.97 for the $A$ - and 0.94 for the $A_{S}$-optimality criterion. Differences in minimum relative efficiency between $\mathrm{ABN}$ and ANBN were mostly small for given SOA and block length. For $A$ - and $A_{S}$-optimality criteria and for $D$ - and $D_{S}$-optimality criteria, it can be concluded from Figures 2.4a-2.4d that the designs with null blocks performed generally better than $\mathrm{AB}$ for any given SOA with little difference between ABN and ANBN. Furthermore, the effect of block length on minimum relative efficiencies was not large. 


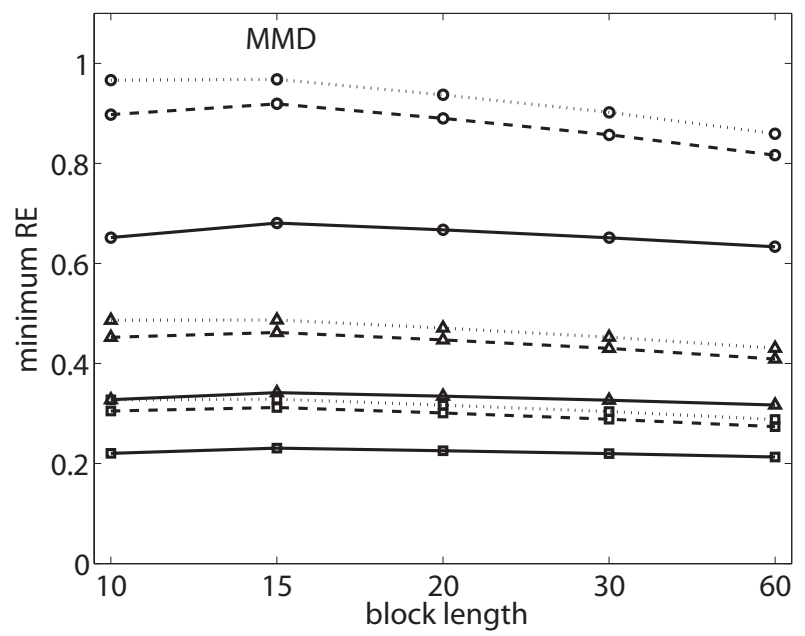

(a) $D$-optimality criterion

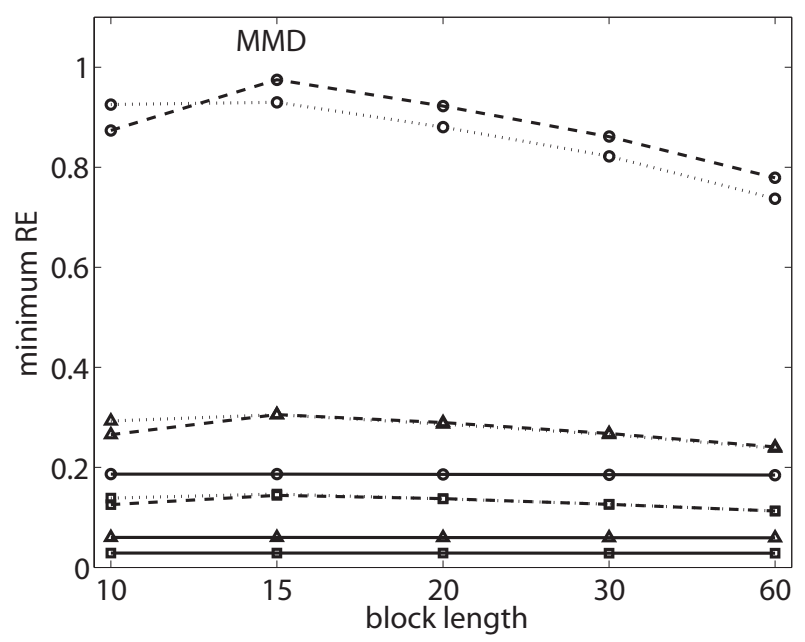

(c) A-optimality criterion

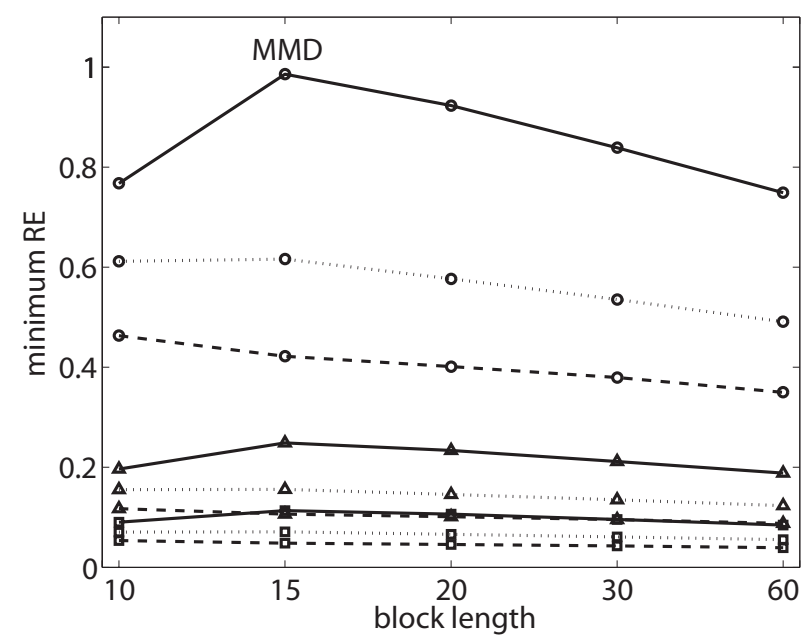

(e) c-optimality criterion

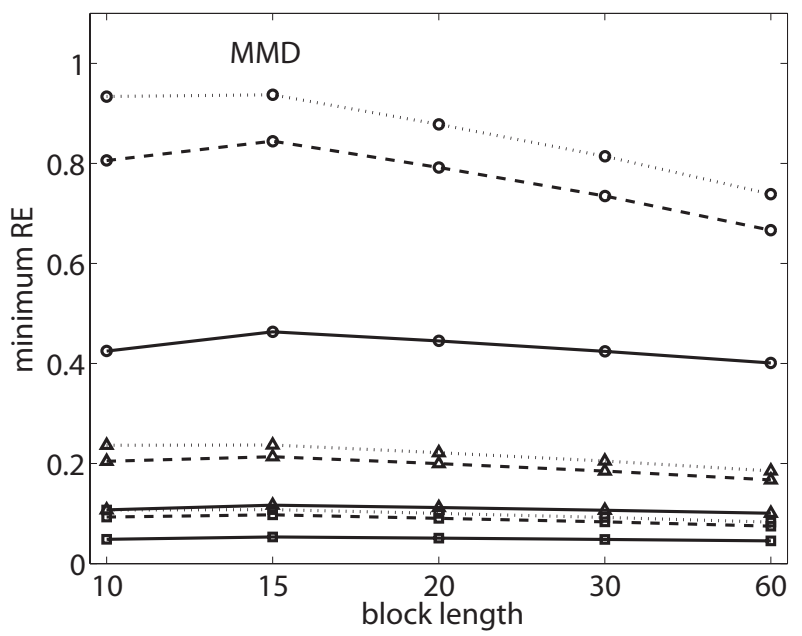

(b) $D_{S}$-optimality criterion

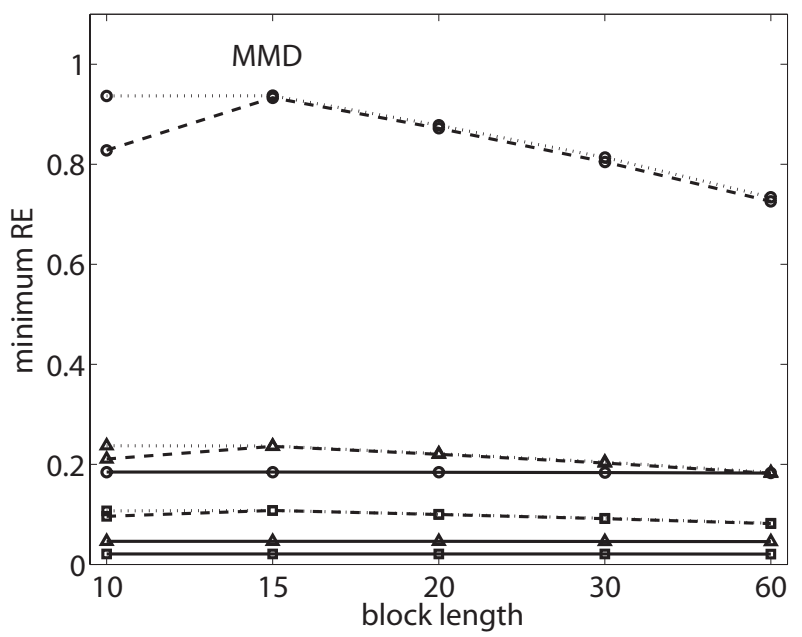

(d) $A_{S}$-optimality criterion

Figure 2.4: Minimum relative efficiencies with AR1. MMD denotes the maximin design. 
c-optimality criterion. Figure $2.4 \mathrm{e}$ shows that the maximin design had order $\mathrm{AB}$, $\mathrm{SOA}=1 \mathrm{~s}$, block length $15 \mathrm{~s}$, and a maximin value of 0.99 . Depending on $\rho$, the designs with $\mathrm{SOA}=1 \mathrm{~s}, \mathrm{BL}=15 \mathrm{~s}$ or $20 \mathrm{~s}$, and order $\mathrm{AB}$ were locally optimal. Block order $\mathrm{AB}$ was for any given block length and SOA more efficient than block order ABN, which in turn was more efficient than block order ANBN. The minimum relative efficiencies increased from block length $10 \mathrm{~s}$ to $15 \mathrm{~s}$ and decreased for higher block lengths than $15 \mathrm{~s}$, except for order ANBN.

\subsubsection{Costs of choosing nonoptimal design factors}

For the $A$-optimality criterion, the maximin design $(\mathrm{BL}=15 \mathrm{~s}, \mathrm{SOA}=1 \mathrm{~s}, \mathrm{BO}=\mathrm{ANBN})$ had a minimum relative efficiency of almost 1, whereas changing either block order or SOA to the least optimal value leads to minimum relative efficiencies smaller than 0.2 . To exemplify the effect of choosing nonoptimal design factors in terms of costs, the results from the $A$-optimality criterion are therefore used as an extreme case. If the SOA of the maximin design is changed from $1 \mathrm{~s}$ to $3 \mathrm{~s}$, the minimum relative efficiency becomes 0.14 in Figure 2.4c. This means that $1 / 0.14 \approx 7.14$ replications (seven runs) of this design $(\mathrm{SOA}=3 \mathrm{~s}, \mathrm{BL}=15 \mathrm{~s}$, block order $=\mathrm{ANBN})$ are needed to be approximately as efficient as the maximin design. Assuming costs of $\$ 600$ per hour, $\$ 875$ instead of $\$ 125$ would have to be spent to obtain the same efficiency.

If the block order is changed to $\mathrm{AB}$ keeping $\mathrm{SOA}=1 \mathrm{~s}$ and $\mathrm{BL}=15 \mathrm{~s}$, it can be calculated that about $1 / 0.19 \approx 5.26$ replications (five runs) are necessary to be nearly as efficient as the maximin design. With the same assumption of costs per hour as before, we obtain costs of $\$ 625$ instead of $\$ 125$. By changing the block length of the maximin design to $60 \mathrm{~s}$, a minimum relative efficiency of 0.87 is obtained in Figure $2.4 \mathrm{c}$ and $1 / 0.78 \approx 1.28$ repetitions. Thus, the design with $\mathrm{BL}=60 \mathrm{~s}$ is almost as efficient as the maximin design. In conclusion, SOA and block order had a strong effect on costs, whereas block length had a weak effect on costs for the $A$-optimality criterion.

\subsubsection{Effect of nonlinearity}

Based the on results by Binder et al. (1994), the effect of nonlinearity on design efficiency was studied by assuming that the amplitude of the HRF was reduced to $81 \%$ of its canonical value in case of $\mathrm{SOA}=1 \mathrm{~s}$, while $\mathrm{SOA}=2 \mathrm{~s}$ or $3 \mathrm{~s}$ were assumed to give rise to the canonical HRF. For the $A_{-}^{-}, D_{S^{-}}, A_{S^{-}}$and $c$-optimality criteria, the minimum relative efficiency of $\mathrm{SOA}=2 \mathrm{~s}$ was $38 \%-44 \%$ of the minimum relative efficiency of the same design with $\mathrm{SOA}=1$ s (block length and block order not changed), instead of $25 \%-33 \%$ for linearity. For the $D$-optimality criterion, a design with $\mathrm{SOA}=2 \mathrm{~s}$ had $62 \%$ instead of $50 \%$ of the minimum relative efficiency of a design with $\mathrm{SOA}=1 \mathrm{~s}$. For the $A_{-}^{-}, D_{S^{-}}, A_{S^{-}}$and $c$-optimality criteria, $\mathrm{SOA}=3 \mathrm{~s}$ resulted in $17 \%-21 \%$ of the minimum relative efficiency of $\mathrm{SOA}=1 \mathrm{~s}$ instead of $11 \%-16 \%$ for linearity while for the $D$-optimality criterion, this value was between $41 \%$ to $42 \%$ instead of $34 \%$. In conclusion, an SOA equal to $1 \mathrm{~s}$ was still the most efficient, but the effect of the SOA on design efficiency was less strong if nonlinearity was taken into account. 


\subsubsection{Uncorrelated error and autoregressive error plus measure- ment error}

For uncorrelated errors, the maximin approach is not needed because $\rho=0$ and the optimal design can be determined by comparing the relative efficiencies directly. The results were similar to those of AR1 with two mentionable differences. Firstly, $A$-optimal was the design with $\mathrm{SOA}=1 \mathrm{~s}$, block order $\mathrm{ANBN}$, and $\mathrm{BL}=20 \mathrm{~s}$ instead of $15 \mathrm{~s}$. Secondly, $c$-optimal was the design with $\mathrm{SOA}=1 \mathrm{~s}$, order $\mathrm{AB}$, and $\mathrm{BL}=20 \mathrm{~s}$ instead of $15 \mathrm{~s}$, as can be seen in Table 2.1.

Table 2.1: Maximin designs for AR1 and AR1+ME and the optimal designs for uncorrelated errors (UE)

\begin{tabular}{|c|c|c|c|}
\hline \multirow[b]{2}{*}{$\begin{array}{l}\text { Optimality } \\
\text { criterion }\end{array}$} & \multicolumn{3}{|c|}{ Error structure } \\
\hline & AR1 & UE & $\mathrm{AR} 1+\mathrm{ME}$ \\
\hline$D$ & $\begin{array}{l}\mathrm{SOA}=1 \mathrm{~s}, \mathrm{BL}=15 \mathrm{~s}, \\
\text { order }=\mathrm{ABN}\end{array}$ & $\begin{array}{l}\mathrm{SOA}=1 \mathrm{~s}, \mathrm{BL}=15 \mathrm{~s}, \\
\text { order }=\mathrm{ABN}\end{array}$ & $\begin{array}{l}\mathrm{SOA}=1 \mathrm{~s}, \mathrm{BL}=10 \mathrm{~s}, \\
\text { order }=\mathrm{ABN}\end{array}$ \\
\hline$D_{S}$ & $\begin{array}{l}\mathrm{SOA}=1 \mathrm{~s}, \mathrm{BL}=15 \mathrm{~s}, \\
\text { order }=\mathrm{ABN}\end{array}$ & $\begin{array}{l}\mathrm{SOA}=1 \mathrm{~s}, \mathrm{BL}=15 \mathrm{~s}, \\
\text { order }=\mathrm{ABN}\end{array}$ & $\begin{array}{l}\mathrm{SOA}=1 \mathrm{~s}, \mathrm{BL}=10 \mathrm{~s}, \\
\text { order }=\mathrm{ABN}\end{array}$ \\
\hline$A$ & $\begin{array}{l}\mathrm{SOA}=1 \mathrm{~s}, \mathrm{BL}=15 \mathrm{~s}, \\
\text { order }=\mathrm{ANBN}\end{array}$ & $\begin{array}{l}\mathrm{SOA}=1 \mathrm{~s}, \mathrm{BL}=20 \mathrm{~s} \\
\text { order }=\mathrm{ANBN}\end{array}$ & $\begin{array}{l}\mathrm{SOA}=1 \mathrm{~s}, \mathrm{BL}=15 \mathrm{~s}, \\
\text { order }=\mathrm{ANBN}\end{array}$ \\
\hline$A_{S}$ & $\begin{array}{l}\mathrm{SOA}=1 \mathrm{~s}, \mathrm{BL}=15 \mathrm{~s}, \\
\text { order }=\mathrm{ABN}\end{array}$ & $\begin{array}{l}\mathrm{SOA}=1 \mathrm{~s}, \mathrm{BL}=15 \mathrm{~s} \\
\text { order }=\mathrm{ABN}\end{array}$ & $\begin{array}{l}\mathrm{SOA}=1 \mathrm{~s}, \mathrm{BL}=10 \mathrm{~s}, \\
\text { order }=\mathrm{ABN}\end{array}$ \\
\hline$c$ & $\begin{array}{l}\mathrm{SOA}=1 \mathrm{~s}, \mathrm{BL}=15 \mathrm{~s}, \\
\text { order }=\mathrm{AB}\end{array}$ & $\begin{array}{l}\mathrm{SOA}=1 \mathrm{~s}, \mathrm{BL}=20 \mathrm{~s}, \\
\text { order }=\mathrm{AB}\end{array}$ & $\begin{array}{l}\mathrm{SOA}=1 \mathrm{~s}, \mathrm{BL}=15 \mathrm{~s}, \\
\text { order }=\mathrm{AB}\end{array}$ \\
\hline
\end{tabular}

The same locally optimal designs as for AR1 were obtained for AR1+ME, except for the $c$-optimality criterion, which had locally optimal designs with block lengths $10 \mathrm{~s}, 15 \mathrm{~s}$, $20 \mathrm{~s}$, block order $\mathrm{AB}$, and $\mathrm{SOA}=1 \mathrm{~s}$. Block length $10 \mathrm{~s}$ performed better than for AR1, as can be seen in Table 2.1. Furthermore, the effect of block length was slightly stronger than for AR1 with a stronger decrease in minimum relative efficiencies for higher block length. Similarly to AR1, lower SOAs performed better than higher SOAs, and null blocks were preferred by the $D-, D_{S^{-}}, A$ - and $A_{S^{-}}$optimality criteria, whereas no null blocks were preferred by the $c$-optimality criterion.

Depending on both the ratio $\sigma^{2} / \sigma_{2}^{2}$ and the autocorrelation parameter $\rho$, the locally optimal design differed in block length. However, the locally optimal designs had always an SOA equal to $1 \mathrm{~s}$ and block length 10, 15 or 20 seconds for all optimality criteria. The locally optimal designs had block order $\mathrm{ABN}$ for the $D^{-}, D_{S^{-}}$and $A_{S^{-}}$optimality criteria, block order ANBN for the $A$-optimality criterion, and block order AB for the $c$-optimality criterion. The maximin designs can be seen in Table 2.1. For higher ratios of the variances $\sigma^{2} / \sigma_{2}^{2}$, AR1+ME behaved more similar to AR1 with regard to the locally optimal designs. 


\subsection{Discussion}

The results of AR1 and AR1+ME did not differ much. This was expected as the AR1+ME error structure approaches AR1 for higher ratios $\sigma^{2} / \sigma_{2}^{2}$. Furthermore, for a smaller ratio of the variances, the AR1+ME structure is approximating uncorrelated errors, and UE gave also similar results as AR1. Therefore, the further discussion of results is restricted to the AR1 error structure.

The $D^{-}, D_{S^{-}}$and $A_{S^{-}}$optimality criteria gave very similar results with respect to the maximin design and the evaluation of block order ABN and ANBN. For all these optimality criteria, the maximin design was the design with $\mathrm{SOA}=1 \mathrm{~s}$, block length=15 $\mathrm{s}$ and block order ABN. Furthermore, block order ABN performed always slightly better than ANBN for a given SOA. The maximin designs for the $A$ - and $c$ - optimality criteria differed only in block order: ANBN for $A$ - and AB for the $c$-optimality criterion.

The preference for block order $\mathrm{AB}$ of the $c$-criterion is in agreement with Smith et al. (2007), who showed that for estimating a contrast between two task blocks no rest period (no null block) is optimal. Similarly, Friston et al. (1999) found that for event-related designs, no null events should be used to estimate the differential contrast. In contrast, designs with null blocks were favored by the $D$-, $D_{S^{-}}, A$ - and $A_{S^{-}}$optimality criteria as null blocks are necessary for the estimation of $\beta_{0}, \beta_{1}$ and $\beta_{2}$.

Regarding SOA, we found that small SOAs resulted in higher values of the minimum relative efficiencies if the other design factors are constant. This was expected because more trials in one block cause an overlap of the hemodynamic responses leading to a higher total response which simplifies the detection of activated areas. However, the magnitude of the effect of SOA was larger than expected, e.g., for all optimality criteria except the $D$ optimality criteria, the minimum relative efficiency decreased from $\mathrm{SOA}=1 \mathrm{~s}$ to $\mathrm{SOA}=2 \mathrm{~s}$ by at least $67 \%$ ( $D$-optimality criterion: exactly $50 \%$ ) under linearity.

Optimal block lengths were $10 \mathrm{~s}, 15 \mathrm{~s}$ or $20 \mathrm{~s}$, but the effect of block length was small so that the minimum relative efficiency did not change much for different block lengths. The small effect of block length might be explained by the fact that only linear trends were considered, and no other trends or aspects of noise, e.g., physiological noise, were included in the model. On the other hand, the temporally correlated structure of fMRI noise is captured very well by AR1, and results do not differ much between uncorrelated and correlated errors.

A conclusion from the results of the $A_{S^{-}}$and $D_{S^{-}}$optimality criteria is that null blocks are recommendable to estimate the stimulus effects. To estimate the contrast between $\mathrm{A}$ and $\mathrm{B}$, we would advise to apply the block order $\mathrm{AB}$. If there is interest in estimating the stimulus effects and contrasts, order ABN will be a good choice as it outperformed ANBN in the results from the $c$-optimality criterion and performed comparably to ANBN for the other optimality criteria. Optimal stimulus onset asynchrony (SOA) should be as small as possible, and an optimal block length should be in the range of $10 \mathrm{~s}$ to $20 \mathrm{~s}$. However, the effect of block length was not strong in comparison with the effect of SOA or block order. Thus, in planning an fMRI experiment a researcher may first decide on an appropriate SOA, then on the block order depending on the effects or contrasts of interest, and finally on block length. 
With respect to the choice of optimality criterion, the $A$ - and $A_{S}$-optimality criteria were already applied in fMRI research (Dale, 1999; Liu et al., 2001; Wager \& Nichols, 2003), but there is no reason to generally prefer $A$ - over $D$-optimality concerning the optimization of fMRI experiments. An advantage of the $A$-optimality criterion is that the parameter estimators can be linearly weighted in the trace function (Conlisk \& Watts, 1979) if there is more interest in one parameter than in the other, but that was not the case in this study.

One often cited advantage of the $D$-optimality criterion is the independence of the $D$ optimal design with respect to the scaling of the regressors. Furthermore, the $D$-optimal design results in a minimized confidence ellipsoid for the unknown parameters. A disadvantage is that the $D$-optimal design may lead to minimization of the confidence ellipsoid in only one direction and strong elongation of the ellipse along one axis (Sim \& Roy, 2005; Atkinson \& Donev, 1996, p.106). This can occur if the trials of the two stimuli A and B are not presented equally often or if $\operatorname{Var}(\varepsilon)$ is assumed to be heterogeneous. The comparable results of the $A_{S^{-}}$and $D_{S^{-}}$optimality criteria for the maximin designs and locally optimal designs may be explained by the homogeneity of the error variance and the fact that the regressors for stimulus effects were time-shifted versions of each other. Modeling heterogeneity or unequal number of trials is, however, beyond the scope of this paper.

So, for this study there is no obvious choice between the $A$ - or $D$-optimality criteria. We do, however, recommend restricting to the optimization of the stimulus effects' estimation, in particular if more confounders are included in the model, e.g., quadratic trend effects. Thus, the following optimality criteria remain: the $c$-optimality criterion for estimation of contrasts and the $A_{S^{-}}$or $D_{S^{-}}$optimality criteria. Since the $D_{S^{-}}$optimality criterion results in a minimized confidence ellipsoid of the estimators of interest, we prefer this optimality criterion for our modeled experiment.

Further research is needed to determine to which extent the results depend on the parameterization of the double gamma function. For example, the optimal block length might increase if the hemodynamic response function is more broad, and thus the power spectrum of the HRF is higher at low frequencies. Liu et al. (2001) showed this for uncorrelated errors, but more research also with correlated errors is needed. Furthermore, the optimal block order could differ if the length and the strength of the undershoot is changed, e.g., more null blocks might be necessary for a longer undershoot.

Another important aspect for further research is the extension of our model to mixedeffects models and the optimization of designs for second level analysis, which is nowadays common in fMRI data analysis. Random effects are necessary to generalize inferences to the population from which subjects are drawn. With regard to optimization of designs for these models, the covariance matrix $\operatorname{Cov}(\hat{\beta})$ in Eq. (2.7) has to be adjusted by taking inter-subject and intra-subject variance into account. 
Chapter 2 


\section{CHAPTER 3}

\section{Robustness of optimal design of fMRI experiments with application of a genetic algorithm}

This chapter is published in NeuroImage (2010), 49: 2433-2443, with co-authors Gerard J. P. van Breukelen, Rainer Goebel and Martijn P. F. Berger. 


\begin{abstract}
In this paper we apply the genetic algorithm developed by Kao et al. (2009) to find designs which are robust against misspecification of the error autocorrelation. Two common optimality criteria, the $A$-optimality criterion and the $D$-optimality criterion, based upon a general linear model are employed to obtain locally optimal designs for a given value of the autocorrelation. The maximin criterion is then used to obtain designs which are robust against misspecification of the autocorrelation. Furthermore, robustness depending on the choice of optimality criterion is evaluated. We show analytically and empirically that the $A$ - and $D$-optimality criterion will result in different optimal designs, e.g., with different stimulus frequencies. Optimal stimulus frequency for the $A$-optimality criterion has been derived by Liu \& Frank (2004) whereas we derive here the optimal stimulus frequency for the $D$-optimality criterion. Conclusions about the robustness of an optimal design against misspecification of model parameters and choice of optimality criterion are drawn based upon our results.
\end{abstract}

Keywords: fMRI, efficiency, robustness, maximin, $A$-optimality, $D$-optimality 


\subsection{Introduction}

To perform an fMRI experiment, a stimulus sequence of experimental stimuli has to be chosen for presentation to the subject in the scanner. The challenge is to find an optimal sequence out of the many possible sequences (Hagberg et al., 2001; Buračas \& Boynton, 2002). A sequence can be optimal for detection power or for estimation efficiency (Birn et al., 2002; Liu et al., 2001; Liu \& Frank, 2004). The former refers to the detection of brain activation and the latter to the estimation of the hemodynamic response function (HRF). The HRF describes the fMRI signal evoked by one short, brief stimulus.

It has been shown that estimation efficiency and detection power cannot be optimized by the same design (Birn et al., 2002; Liu et al., 2001; Liu \& Frank, 2004). Blocked designs where stimuli of the same type are presented within blocks are known to be optimal for detection power (Bandettini \& Cox, 2000; Friston et al., 1999; Smith et al., 2007) whereas rapid event-related designs characterized by a random order of stimuli and varying interstimulus interval (ISI) have been shown to be optimal for estimation efficiency (Birn et al., 2002; Chein \& Schneider, 2003; Liu et al., 2001; Liu \& Frank, 2004). Optimal designs will also vary depending on the contrast of interest, e.g., detection of activation of individual stimuli or detection of activation of stimuli contrasts (Friston et al., 1999; Smith et al., 2007; Kao et al., 2009). Furthermore, psychological aspects should be taken into account, e.g., anticipation of stimuli by subjects has to be avoided. Counterbalancing the order of trials in a design is a method to prevent such stimulus anticipation by subjects. Additionally to psychological aspects, a researcher might have restrictions on the stimulus frequencies in the design.

Kao et al. (2009) have developed a flexible tool based on a genetic algorithm (GA) which can take all these aspects into account and generate optimal designs for four different design criteria: detection power, estimation efficiency, counterbalancing and desired stimulus frequency. These four criteria can be weighted depending on their relevance for a given experiment to generate a multi-objective design criterion (MO-criterion). A detailed description of the application of genetic algorithms to fMRI experimental design can be found in Kao et al. (2007, 2009) and Wager \& Nichols (2003). The optimal designs obtained by Kao et al. (2009) were superior to the designs proposed by the genetic algorithm of Wager \& Nichols (2003) and to $m$-sequences (Buračas \& Boynton, 2002). Furthermore, Kao et al. (2009) showed that their algorithm performed the computations faster than the algorithm of Wager \& Nichols (2003). Thus, we used the genetic algorithm of Kao et al. (2009) to obtain locally optimal designs. Furthermore, we will extend their results by considering a broader range of autocorrelation and by using the $A$ - as well as the $D$-optimality criterion (Atkinson et al., 2007).

Kao et al. (2009) modeled the temporally correlated noise which is typically observed in fMRI studies by an autoregressive error of order 1 (AR1) with 0.3 correlation. AR1 is a common and justified model for fMRI data (Bullmore et al., 1996; Gautama \& Van Hulle, $2005)$ but the autocorrelation in the AR1 model can still vary between experiments and might therefore influence the optimal design. The autocorrelation may also vary from voxel to voxel or from region of interest (ROI) to region of interest, so that an optimal design calculated for a given autocorrelation of a voxel or ROI might not be optimal for another 
voxel or another ROI.

In this paper, we therefore generate optimal designs by the genetic algorithm of Kao et al. (2009) and evaluate the robustness of these designs with regards to the autocorrelation in the time series and choice of optimality criterion, i.e., $A$ - and $D$-optimality criterion. We answer the question whether the obtained designs are still efficient for the whole range of possible autocorrelation values and for both optimality criteria. Robustness of a design is an important property as robust designs are less dependent on the assumptions upon which they are obtained and have a high overall efficiency. Therefore, we use the maximin criterion (Berger \& Tan, 2004) to accommodate for the unknown value of the autocorrelation and obtain designs which are robust against misspecification of this value. We compare different locally optimal designs for a realistic range of the autocorrelation and determine the maximin design which will be independent of the specific autocorrelation value. As far as we know, designs which are robust against misspecification of a certain parameter have not yet been studied for fMRI experiments.

The $A$-optimality criterion has often been used to find optimal designs for detection power and estimation efficiency (Dale, 1999; Wager \& Nichols, 2003; Liu et al., 2001; Kao et al., 2009) but the $D$-optimality which is a popular criterion in experimental design has not yet been very often applied in optimization of fMRI experiments. One advantage of the $D$-optimality criterion is that the $D$-optimal design will result in a minimized confidence ellipsoid for the unknown parameters if the errors are normally distributed or in a minimized asymptotic confidence ellipsoid if the number of time points is sufficiently large. Furthermore, the $D$-optimality criterion is independent of the scale of the regressors and therefore advantageous in some situations while in other situations the $A$-optimality criterion might be preferable. The $A$ - and $D$-optimality criterion can result in different optimal designs and so the differences and similarities between an obtained $A$ - and $D$-optimal design should be examined. We will compare $A$-optimal designs with $D$-optimal designs to see how the $A$-optimal designs perform with respect to the $D$-optimality criterion and vice versa.

In Section 3.2 we will introduce the methodology, that is the applied linear models in Section 3.2.1, the design criteria and optimality criteria in Section 3.2.2 as well as the maximin criterion in Section 3.2.3. Furthermore, we will explain the simulations which we performed in Section 3.2.4, and the results of the simulations are given in Section 3.3. Section 3.3 also presents some theoretical results on the optimal stimulus frequency. Finally, we will discuss our results and draw conclusions in Section 3.4.

\subsection{Methodology}

\subsubsection{Linear models for estimation efficiency and detection power}

We consider two common linear models for fMRI data (Friston et al., 1995; Dale, 1999; Kao et al., 2009). The first model is used for estimating the hemodynamic response function 
and the second model is used for detecting activation:

$$
\begin{aligned}
& Y=X h+S \gamma+\epsilon=\left[\begin{array}{ll}
X & S
\end{array}\right]\left(\begin{array}{l}
h \\
\gamma
\end{array}\right)+\epsilon, \text { and } \\
& Y=Z \theta+S \gamma+\eta=\left[\begin{array}{ll}
Z & S
\end{array}\right]\left(\begin{array}{l}
\theta \\
\gamma
\end{array}\right)+\eta,
\end{aligned}
$$

where $Y$ is the observed voxel-wise fMRI signal, $X=\left[X_{1} \ldots X_{Q}\right]$ is the design matrix and $h=\left(h_{1}^{\prime}, \ldots, h_{Q}^{\prime}\right)$ is a vector of unknown parameters for the HRFs of the $Q$ stimulus types (Dale, 1999). Each matrix $X_{i}$ is an $N \times k$ matrix where $N$ is the number of time points and $k$ gives the number of time points per hemodynamic response function. The second model (Friston et al., 1995) describes the observed fMRI signal response $Y$ by a design matrix $Z$ based on the assumed hemodynamic response and unknown parameters $\theta$ which represent the amplitude of the hemodynamic response. Both models contain a nuisance design matrix $S$ and unknown nuisance parameters $\gamma$ to account, e.g., for a constant baseline signal and for scanner drift. The errors $\epsilon$ and $\eta$ are assumed to follow an AR1 structure with $\Sigma$ being the covariance matrix of the errors and $V=\Sigma^{-1 / 2}$ is a whitening matrix such that $V \epsilon$ and $V \eta$ are white noise.

Let $X_{\perp}=\left(I-P_{V S}\right) V X$ and $Z_{\perp}=\left(I-P_{V S}\right) V Z$ with $I$ the identity matrix of dimension $N, P_{V S}=V S\left[(V S)^{T} V S\right]^{-}(V S)^{T}$ and $A^{-}$the generalized inverse of $A$ (Liu \& Frank, 2004; Kao et al., 2009). Then the covariance matrix of the generalized least squares (GLS) estimators $\hat{h}$ and $\hat{\theta}$ equals

$$
\begin{aligned}
& \operatorname{Cov}(\hat{h})=\left(X_{\perp}^{T} X_{\perp}\right)^{-} \\
& \operatorname{Cov}(\hat{\theta})=\left(Z_{\perp}^{T} Z_{\perp}\right)^{-} .
\end{aligned}
$$

\subsubsection{Design criteria and optimality criteria}

We consider the following design criterion for estimation efficiency (Kao et al., 2009):

$$
F_{e}= \begin{cases}r / \operatorname{trace}(\operatorname{Cov}(\hat{h})) & \text { for } A \text {-optimality } \\ \operatorname{det}(\operatorname{Cov}(\hat{h}))^{-1 / r} & \text { for } D \text {-optimality }\end{cases}
$$

where $r$ is the rank of $\operatorname{Cov}(\hat{h})$. For detection power, the following design criterion is applied (Kao et al., 2009):

$$
F_{d}= \begin{cases}r_{c} / \operatorname{trace}(\operatorname{Cov}(C \hat{\theta})) & \text { for } A \text {-optimality } \\ \operatorname{det}(\operatorname{Cov}(C \hat{\theta}))^{-1 / r_{c}} & \text { for } D \text {-optimality }\end{cases}
$$

where $\operatorname{Cov}(C \hat{\theta})=C\left(Z_{\perp}^{T} Z_{\perp}\right)^{-} C^{T}$ is the covariance matrix of the estimator for the contrast $C \theta$ and $r_{C}$ is the rank of $C$. For detection of individual stimulus effects, $C$ is the identity matrix of dimension $Q$. The higher the better the two criteria $F_{d}$ and $F_{e}$ are.

The $A$-optimality criterion will be applied with $C$ consisting of all pairwise contrasts, while the $D$-criterion needs a nonsingular covariance matrix of the contrasts and therefore independent linear combinations of the parameters in $C$. Therefore, we will apply linearly 
independent contrasts for the $D$-optimality criterion but not for the $A$-optimality criterion. In contrast to the $D$-optimal design, the $A$-optimal design was dependent on the subset of contrasts chosen when we applied linearly independent contrasts and thus the $A$-optimality criterion needs all pairwise contrasts.

The following two design criteria for counterbalancing and maintenance of desired stimulus frequency are defined on a subdesign excluding null events of the original design. Kao et al. (2009) use the following measure of counterbalancing:

$$
\left.F_{c}=\sum_{r=1}^{R} \sum_{i=1}^{Q} \sum_{j=1}^{Q}\left\lfloor\mid n_{i j}^{r}-(n-r) P_{i} P_{j}\right\rfloor\right\rfloor,
$$

where $n_{i j}^{r}$ is the number of occurrences of a type- $i$ stimulus and type- $j$ stimulus with time lag $r$ between the two stimuli and $n$ is the length of the subdesign. $P_{i}$ is the predefined frequency of stimulus type $i$ in the subdesign and $\lfloor a\rfloor$ for $a \in \mathbb{R}$ is the largest integer not higher than $a$. The criterion $F_{c}$ is in "the lower the better" form.

The fourth criterion is also a "the lower the better" criterion and upholds a desired stimulus frequency (Kao et al., 2009):

$$
F_{f}=\sum_{i=1}^{Q}\left\lfloor\left|n_{i}-n P_{i}\right|\right\rfloor,
$$

where $n_{i}$ is the number of occurrences of stimulus type $i$.

The indexes of the design criteria refer to the criterion which is evaluated: $c$ for counterbalancing, $d$ for detection power, $e$ for estimation efficiency and $f$ for desired stimulus frequency. The following standardization is used to guarantee comparability between the four design criteria:

$$
F_{l}^{*}= \begin{cases}\frac{F_{l}-\min \left(F_{l}\right)}{\max \left(F_{l}\right)-\min \left(F_{l}\right)}, & l=d, e \\ 1-\frac{F_{l}-\min \left(F_{l}\right)}{\max \left(F_{l}\right)-\min \left(F_{l}\right)}, & l=c, f\end{cases}
$$

The maxima of $F_{d}$ respectively $F_{e}$ are obtained by a previous run of the genetic algorithm with weight $w_{d}=1$ respectively $w_{e}=1$ in Eq. (3.10) and with the same experimental conditions whereas the minima of $F_{d}$ and $F_{e}$ are set to zero. The maxima of $F_{c}$ and $F_{f}$ can be calculated from the design where only the stimulus with the lowest desired stimulus frequency occurs and the minima for $F_{c}$ and $F_{f}$ are zero.

A multi-objective design criterion (MO-criterion) is element of the family of MO-criteria given by

$$
\left\{F^{*}=w_{c} F_{c}^{*}+w_{d} F_{d}^{*}+w_{e} F_{e}^{*}+w_{f} F_{f}^{*}: w_{l} \geq 0, l=c, d, e, f ; \sum_{l} w_{l}=1\right\},
$$

where the weights can be selected by the researcher. The MATLAB code provided by Kao et al. (2009) at http://www.stat.uga.edu/ amandal/ can be used to find an optimal MO-design via a genetic algorithm.

\subsubsection{Maximin criterion}

The design criteria for estimation efficiency and detection power, respectively the whitening matrix $V$, depend on the assumed noise structure for the error terms. We assume that the 
errors have an AR1 structure and are normally distributed. This means that $\operatorname{Cov}\left(\epsilon_{i}, \epsilon_{j}\right)=$ $\sigma^{2} \rho^{|i-j|}$ with $\sigma^{2}>0$ and $|\rho|<1$. The optimal design found by the genetic algorithm can only be locally optimal, i.e., for given autocorrelation $\rho$. However, we want to compare designs for different values of the autocorrelation and determine a design which is robust against misspecification of the autocorrelation parameter. This can be done by calculating the maximin design based on a relative efficiency measure.

The relative efficiency of a design $\xi_{1}$ versus a design $\xi_{2}$ is defined as:

$$
\operatorname{RE}\left(\xi_{1} \mid \xi_{2}\right)=\frac{F^{*}\left(\xi_{1}\right)}{F^{*}\left(\xi_{2}\right)}
$$

For a fixed value of autocorrelation $\rho$ a locally optimal design $\xi_{\rho}^{*}$ can be obtained. Since the interval $[0,0.5]$ includes commonly observed values of autocorrelation for fMRI data, given a sampling rate/repetition time (TR) of $2 \mathrm{~s}$, our design space $\Xi$ consists of all the locally optimal designs $\xi^{*}$ for $\rho \in[0,0.5]$. The maximin design MMD will be chosen from this design space. The maximin design MMD is the design which fulfills the following condition:

$$
\operatorname{MMD}=\arg \max _{\xi^{*} \in \Xi} \min _{\rho \in[0,0.5]} \operatorname{RE}\left(\xi^{*} \mid \xi_{\rho}^{*}\right) .
$$

\subsubsection{Simulations}

The first simulation was done to maximize detection power and to examine the efficiency of the locally optimal designs depending on the autocorrelation and the robustness of the maximin design against misspecification of the autocorrelation. The same purpose for estimation efficiency was pursued by the second simulation. The third and fourth simulation concern optimizing several design criteria simultaneously via the MO-criterion and studying the robustness of locally optimal MO-designs. For this purpose, we chose an MO-criterion with equal weights for detection power and for estimation efficiency in simulation III. Furthermore in simulation IV, one MO-criterion with equal weights for all four design criteria was considered.

For all simulations we assumed a second order Legendre polynomial for the nuisance matrix $S$ and an AR1 error structure. When we refer to stimuli, we are not including null events. The number of stimulus types was set to three $(Q=3)$ and $N=255$ events were considered so that generation of $m$-sequences which are restricted to a certain number of stimulus types and sequence lengths was technically possible. The stimuli will be denoted by $\mathrm{A}, \mathrm{B}$ or $\mathrm{C}$ for the stimulus events and by 0 for null events. For the ISI (interstimulus interval) and the TR $2 \mathrm{~s}$ were applied. This means that the minimum ISI is $2 \mathrm{~s}$ and random ISIs which are a multiple of $2 \mathrm{~s}$ are obtained by inclusion of null events due to a rest or fixation condition into the sequence. For the hemodynamic response function the canonical double gamma function which is the sum of two gamma functions was used (Friston et al., 1998). For all simulations, the generation size was 20, number of generations was 10,000, the mutation rate was $1 \%$ and the number of immigrants was four for the genetic algorithm.

Each simulation was performed once for $A$-optimality and once for $D$-optimality. The autocorrelation was varied between 0 and 0.5 in steps of 0.01 . Therefore, in each simulation 
we obtained 51 locally $A$-optimal designs and 51 locally $D$-optimal designs. The following simulations were performed:

- Simulation I with weighting scheme: $w_{d}=1$.

- Simulation Ia for individual stimuli: $C=I_{Q}$, where $I_{Q}$ is the identity matrix of dimension $Q$.

- Simulation Ib for contrasts:

$$
\begin{aligned}
& C_{1}=\left(\begin{array}{ccc}
1 & -1 & 0 \\
0 & 1 & -1 \\
1 & 0 & -1
\end{array}\right) \text { for } A \text {-optimality criterion, } \\
& C_{2}=\left(\begin{array}{ccc}
1 & -1 & 0 \\
0 & 1 & -1
\end{array}\right) \text { for } D \text {-optimality criterion }
\end{aligned}
$$

- Simulation II with weighting scheme: $w_{e}=1, C=I_{Q k}$

- Simulation III with weighting scheme: $w_{d}=w_{e}=0.5, C=I_{Q}$ for detection power and $C=I_{Q k}$ for estimation efficiency.

- Simulation IV with weighting scheme: $w_{c}=w_{d}=w_{e}=w_{f}=0.25, C=I_{Q}$ for detection and $C=I_{Q k}$ for estimation efficiency.

Rest blocks or baseline blocks will be referred to as null blocks. For simulation Ib blocked designs with no null blocks would be optimal (Liu \& Frank, 2004; Liu, 2004; Chein \& Schneider, 2003; Kao et al., 2009; Smith et al., 2007). Therefore, we adapted the code from Kao et al. (2009) to include blocked designs with no null blocks into the possible starting designs. For simulation IV a counterbalancing order of three $(R=3$ in Eq. (3.7)) was applied and the desired stimulus frequency considered equal probability for all stimuli. Null events were not included in the calculation of $F_{f}$ and the desired stimulus frequencies were equal to $1 / 3$ for all stimulus types.

For each simulation the maximin design $\mathrm{MMD}_{A}$ based on the $A$-optimality criterion and the maximin design $\mathrm{MMD}_{D}$ based on the $D$-optimality criterion were determined. By determining subsequently the efficiencies for the $\mathrm{MMD}_{A}$ and $\mathrm{MMD}_{D}$ based on the $D$ optimality criterion respectively $A$-optimality criterion, the $\mathrm{MMD}_{A}$ design can be compared to the $\mathrm{MMD}_{D}$ and vice versa.

The stimulus frequencies of the design were optimized iteratively to maximize detection power or estimation efficiency. We calculated the stimulus frequency for the obtained maximin designs and compared these frequencies to the optimal stimulus frequencies given by Liu \& Frank (2004) for the $A$-optimality criterion and as calculated in Appendix 3.A for the $D$-optimality criterion. More details about the optimal stimulus frequency can be found in Section 3.3.1. 


\subsection{Results}

\subsubsection{Optimal stimulus frequency}

In Appendix 3.A we show that the $D$-optimal stimulus frequency under certain assumptions is equal to $p=1 /(Q+1)$ for detection of individual stimulus effects (detection power) and estimation of individual HRFs (estimation efficiency). Furthermore, $p=1 / Q$ is the $D$-optimal stimulus frequency for $(Q-1)$ linearly independent pairwise contrasts between individual stimuli (detection power) and $(Q-1)$ contrasts between individual HRFs (estimation efficiency). The assumptions are the same as in Liu \& Frank (2004): nonoverlapping trial types, equal number of events per trial type, uncorrelated noise and a nuisance matrix $S$ with a single constant vector. Nonoverlapping trials means that there is only one trial type at each time point.

Liu \& Frank (2004) derived the $A$-optimal stimulus frequency to maximize either detection power or estimation efficiency. If only individual stimuli for detection power or individual HRFs for estimation efficiency are of interest, the $A$-optimal stimulus $p$ frequency is equal to $p=1 /(Q+\sqrt{Q})$ whereas if all pairwise contrasts of stimuli for detection or all pairwise contrasts between individual HRFs are of interest the $A$-optimal frequency $p$ is equal to $p=1 / Q$. The theoretically $A$ - or $D$-optimal stimulus frequencies were compared with those obtained by the GA under AR1 noise and it was found that the obtained frequencies were similar to the theoretically derived values (Tables 3.1-3.4).

\subsubsection{Simulation Ia: Detection power for individual stimuli}

Figure 3.1a shows the unstandardized detection power obtained from all 51 locally $A$ optimal designs for autocorrelation from 0 to 0.5 . Each line gives the detection power $F_{d}$ for one out of 51 locally optimal designs. It can be seen that detection power decreases as the autocorrelation increases. For locally optimal designs for autocorrelation $0.47 \leq \rho \leq 0.5$ the detection power is in general lower than for all other locally optimal designs. For the $D$-optimality criterion, the results for detection power are similar to the $A$-optimality criterion as can be seen in Figure 3.1b.

Figure 3.1c shows the minimum relative efficiencies for the 51 locally $A$ - and the 51 locally $D$-optimal designs for detection power. The maximin design $\mathrm{MMD}_{A}$ has a minimum relative efficiency of 0.97 and is the locally optimal design for $\rho=0.3$ while the maximin design $\mathrm{MMD}_{D}$ has a minimum relative efficiency of 0.98 and is obtained for $\rho=0.45$. Figure 3.1c shows that the minimum relative efficiencies are high for all locally optimal designs and both optimality criteria. This can be expected from Figures 3.1a and 3.1b because there is not much variation in detection power between the 51 locally optimal designs for a given autocorrelation so that the minimum relative efficiencies for different designs will be similar. 


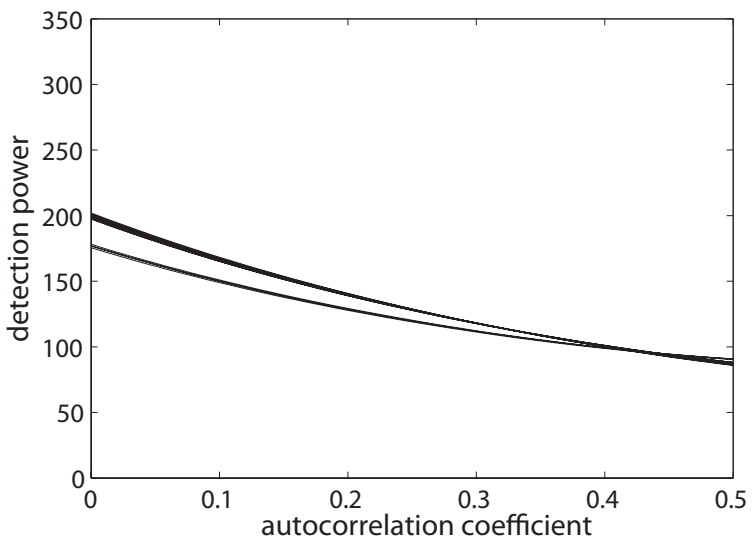

(a) Detection power $F_{d}$ of the 51 locally optimal designs with $A$-optimality criterion, $C=$ $I_{3}$. Each line represents one locally optimal design.

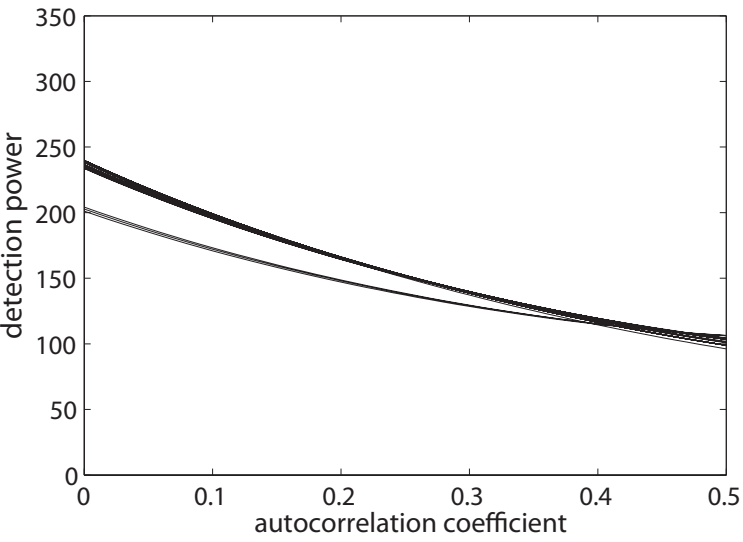

(b) Detection power $F_{d}$ of the 51 locally optimal designs with $D$-optimality criterion, $C=$ $I_{3}$. Each line represents one locally optimal design.

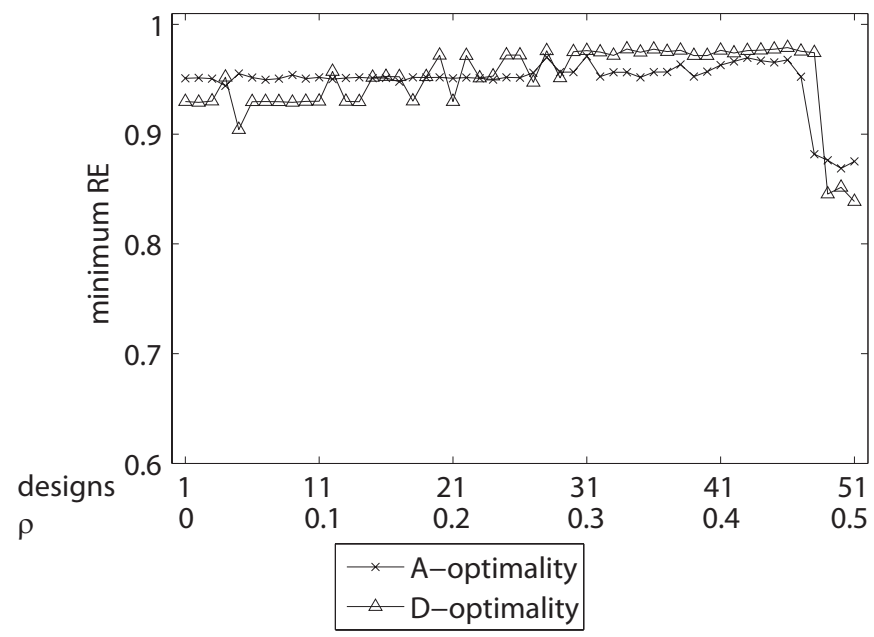

(c) Minimum relative efficiency for detection power $F_{d}, C=I_{3}$. The first design $(x=1$ on the $x$-axis $)$ is the locally optimal design for $\rho=0$, the second design ( $x=2$ on the $x$-axis) is the locally optimal design for $\rho=0.01$ etc.

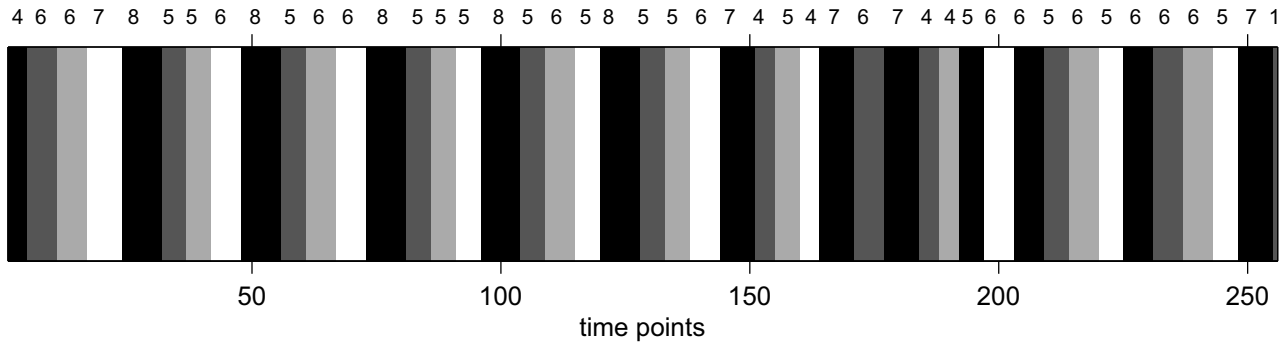

(d) Maximin design for detection power $F_{d}\left(C=I_{3}\right)$ and $A$-optimality. The numbers above blocks indicate the block size (number of trials per block). Block length is equal to block size $\cdot 2 \mathrm{~s}$ as TR was equal to $2 \mathrm{~s}$. The color black corresponds to 0 (null event), dark gray corresponds to stimulus type A, light gray corresponds to stimulus type B and white corresponds to stimulus type C.

Figure 3.1: Results for detection power $F_{d}$ of individual stimulus effects for different optimality criteria 
Table 3.1: Maximin designs for detection power with their mean block lengths, stimulus frequencies and theoretically optimal stimulus frequencies. Overall F-tests based on a oneway ANOVA were performed to compare mean block lengths, and pairwise comparisons between mean block lengths were based on Tukey's honestly significant difference (HSD) $(\alpha=0.05)$.

(a) Mean block lengths and stimulus frequencies of maximin design for detection power with $A$-optimality criterion

\begin{tabular}{cccc}
\hline \hline Stimulus & Mean block length & Frequency & Theoretical \\
\hline 0 & $13.69 \mathrm{~s}$ & 0.35 & 0.37 \\
$\mathrm{~A}$ & $9.50 \mathrm{~s}$ & 0.22 & 0.21 \\
$\mathrm{~B}$ & $10.80 \mathrm{~s}$ & 0.21 & 0.21 \\
$\mathrm{C}$ & $11.00 \mathrm{~s}$ & 0.22 & 0.21 \\
\hline \multicolumn{4}{c}{ Overall F-test: $p<0.001$} \\
\hline \hline \multicolumn{4}{c}{ Pairwise comparisons (Tukey's HSD) } \\
\hline Stimulus 1 & Stimulus 2 & $p$-value \\
\hline \multicolumn{5}{c}{} \\
$\mathrm{A}$ & $\mathrm{A}$ & $<0.001$ \\
$\mathrm{~B}$ & $\mathrm{~B}$ & 0.018 \\
$\mathrm{~B}$ & $\mathrm{~B}$ & 0.031 & \\
\hline \hline
\end{tabular}

(b) Mean block lengths and stimulus frequencies of maximin design for detection power with $D$-optimality criterion

\begin{tabular}{cccc}
\hline \hline Stimulus & Mean block length & Frequency & Theoretical \\
\hline 0 & $10.50 \mathrm{~s}$ & 0.25 & 0.25 \\
$\mathrm{~A}$ & $11.82 \mathrm{~s}$ & 0.25 & 0.25 \\
$\mathrm{~B}$ & $12.40 \mathrm{~s}$ & 0.24 & 0.25 \\
$\mathrm{C}$ & $11.82 \mathrm{~s}$ & 0.25 & 0.25 \\
\hline \multicolumn{4}{c}{ Overall F-test: $p=0.283$} \\
\hline \hline
\end{tabular}

The locally optimal designs for higher autocorrelations perform slightly worse than the other locally optimal designs in Figure 3.1c. This may be explained by the fact that for these higher autocorrelation values the genetic algorithm selected a different blocked design as starting design out of 22 possible blocked designs based on detection power. Optimization of detection power depends strongly on good starting designs and is only with difficulty improved by random mutation, immigration and crossover.

The maximin design $\mathrm{MMD}_{A}$ for detection power is presented in Figure 3.1d showing that the design is a blocked design with a pattern of ABCN except for some deviations from this pattern in the middle of the experiment time which result in more null blocks than stimulus blocks. As can be seen from Table 3.1a, the mean length of null blocks is significantly different from the mean block lengths for the stimulus conditions. Similarly to 
the $A$-optimality criterion, the maximin design $\mathrm{MMD}_{D}$ for detection power was a blocked design and showed an almost consistent ABCN pattern. The mean block lengths based on the $D$-optimality criterion were not significantly different from each other as can be seen in Table 3.1b.

\subsubsection{Simulation Ib: Detection power for contrasts}

Detection power again decreases with increasing autocorrelation $\rho$ as is shown in Figures $3.2 \mathrm{a}$ and $3.2 \mathrm{~b}$. The minimum relative efficiencies are high and the minimum relative efficiencies of those designs which were locally optimal at $\rho$ close to 0 are lower than for the other designs in Figure 3.2c. The reason for this behavior is that the GA selected a different blocked design as starting design for autocorrelations close to zero than for higher autocorrelations. The same maximin design with minimum relative efficiency of 0.98 was obtained for the $A$ - and $D$-optimality criterion. This design was the locally optimal design for an intermediate value of the autocorrelation $\left(\rho_{A}=0.3\right.$ and $\left.\rho_{D}=0.32\right)$ and was a blocked design without null events (see Table 3.2 and Figure 3.2d). Previous studies showed that null events are not necessary to optimize the detection of contrasts (Liu \& Frank, 2004; Friston et al., 1999; Smith et al., 2007).

Table 3.2: Maximin design for detection power of contrasts with $A$ - and $D$-optimality criterion. Mean block lengths, obtained and theoretically optimal stimulus frequencies are presented.

\begin{tabular}{cccc}
\hline Stimulus & Mean block length & Frequency & Theoretical \\
\hline 0 & $0 \mathrm{~s}$ & 0 & 0 \\
$\mathrm{~A}$ & $11.47 \mathrm{~s}$ & 0.34 & 0.33 \\
$\mathrm{~B}$ & $12.00 \mathrm{~s}$ & 0.33 & 0.33 \\
$\mathrm{C}$ & $12.14 \mathrm{~s}$ & 0.33 & 0.33 \\
\hline
\end{tabular}

\subsubsection{Simulation II: Estimation efficiency}

The estimation efficiencies $F_{e}$ for the $A$-optimality criterion and the $D$-optimality criterion are displayed in Figures 3.3a and 3.3b with each line representing the estimation efficiency of one locally optimal design as a function of $\rho$. All designs show an increasing estimation efficiency $F_{e}$ with increasing autocorrelation. The minimum relative efficiencies for the $A$-criterion increase slightly as $\rho$ increases and finally decrease slightly again whereas the minimum relative efficiencies for the $D$-optimality criterion are all almost equal to 1 (see Figure 3.3c).

The maximin designs $\mathrm{MMD}_{A}$ and $\mathrm{MMD}_{D}$ were event-related designs and had a minimum relative efficiency of 0.97 and almost 1 (0.9998). Furthermore, they were locally optimal for $\rho_{A}=0.28$ and $\rho_{D}=0.21$. For the $A$-optimality criterion, a chi-square test on stimulus frequencies including null events rejected the null-hypothesis of equal stimulus frequencies $(p=0.015, \mathrm{df}=3$, Table $3.3 \mathrm{a})$. The stimulus frequencies did not differ significantly from the theoretical values of 0.2113 for stimulus types and 0.3360 for null events 


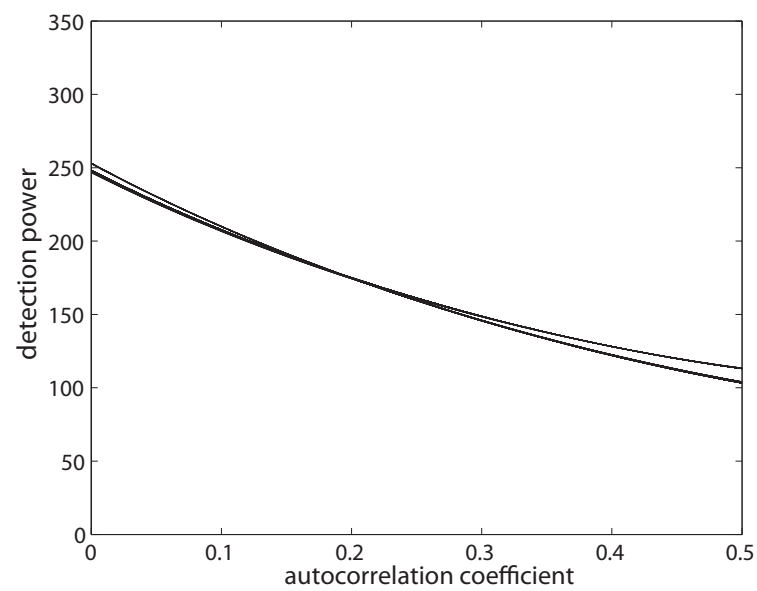

(a) Detection power $F_{d}$ for contrasts with $C_{1}$ of the 51 locally optimal designs with $A$ optimality criterion. Each line represents one locally optimal design.

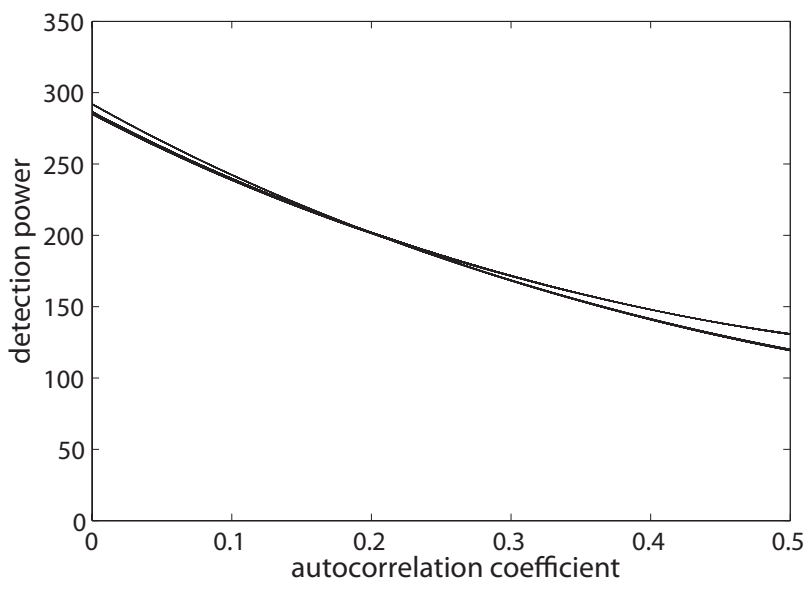

(b) Detection power $F_{d}$ for contrasts with $C_{2}$ of the 51 locally optimal designs with $D$-optimality criterion. Each line represents one locally optimal design.

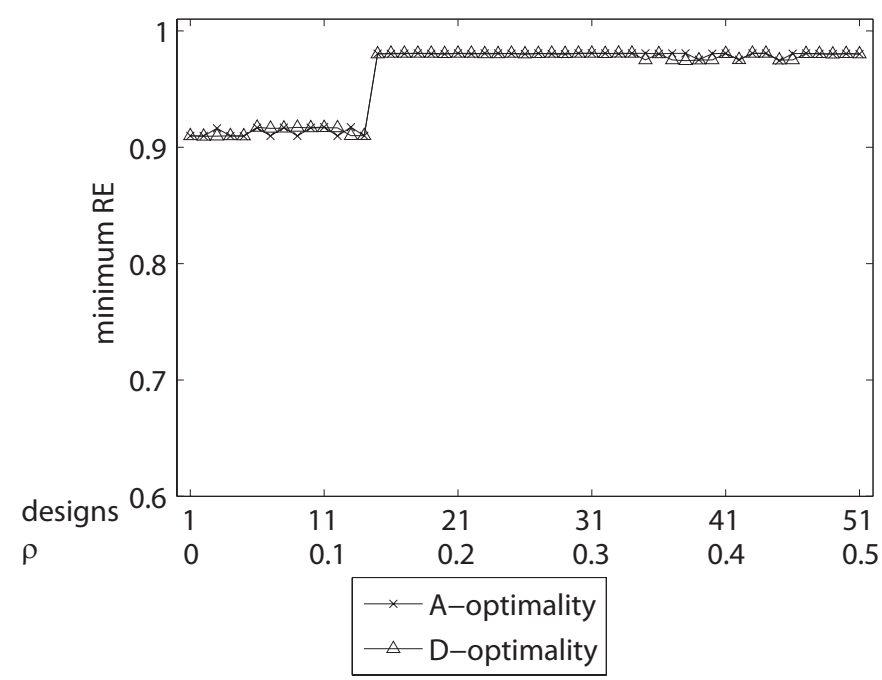

(c) Minimum relative efficiency for detection power $F_{d}$ and for contrasts. The first design $(x=1$ on the $x$-axis $)$ is the locally optimal design for $\rho=0$, the second design $(x=2$ on the $x$-axis) is the locally optimal design for $\rho=0.01$ etc.

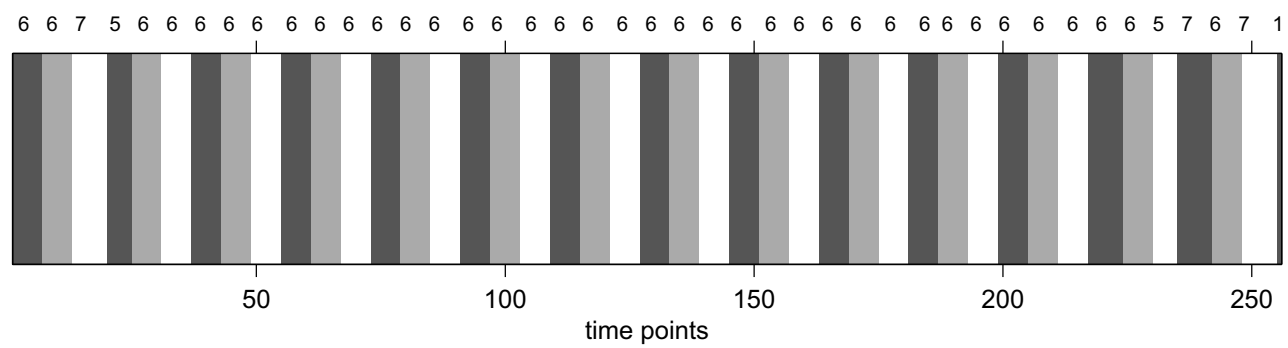

(d) Maximin design for detection power $F_{d}$ of contrasts and $D$-optimality. The numbers above blocks indicate the block size (number of trials per block). Block length is equal to block size $\cdot 2 \mathrm{~s}$ as TR was equal $2 \mathrm{~s}$. The color dark gray corresponds to stimulus type A, light gray corresponds to stimulus type B and white corresponds to stimulus type C.

Figure 3.2: Results for detection power $F_{d}$ of contrasts for different optimality criteria 
$(p=0.797, \mathrm{df}=3$, Table 3.3a, Liu \& Frank, 2004). The null hypothesis of equal stimulus frequencies was not rejected for $\mathrm{MMD}_{D}(p=0.991, \mathrm{df}=3$, Table $3.3 \mathrm{~b})$ which is in line with our theoretical results in Section 3.3.1.

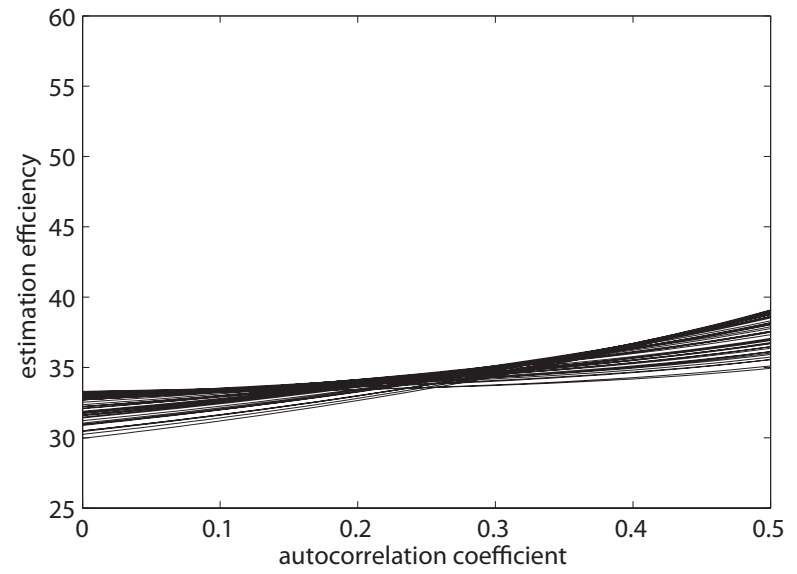

(a) Estimation efficiency $F_{e}$ of the 51 locally optimal designs with $A$-optimality criterion, $C=$ $I_{51}$. Each line represents one locally optimal design.

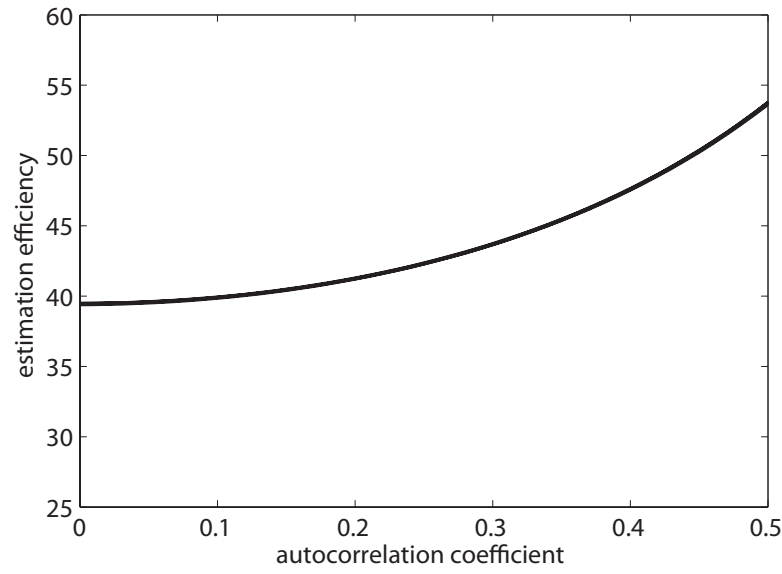

(b) Estimation efficiency $F_{e}$ of the 51 locally optimal designs with $D$-optimality criterion, $C=$ $I_{51}$. Each line represents one locally optimal design.

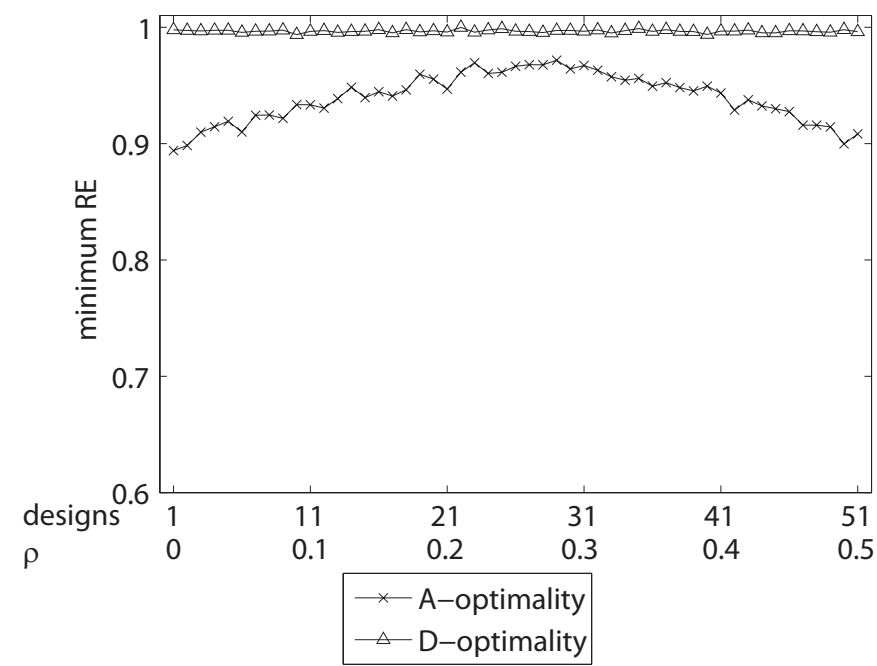

(c) Minimum relative efficiency for estimation efficiency $F_{e}, C=I_{51}$. The first design $(x=1$ on the $x$-axis $)$ is the locally optimal design for $\rho=0$, the second design ( $x=2$ on the $x$-axis) is the locally optimal design for $\rho=0.01$ etc.

Figure 3.3: Results for estimation efficiency $F_{e}$ for different optimality criteria 
Table 3.3: Maximin designs for estimation efficiency with their mean block lengths, stimulus frequencies and theoretically optimal stimulus frequencies. Chi-square tests were performed to test hypotheses about stimulus frequencies $(\alpha=0.05)$. Two null hypotheses were considered: $H_{0}$ : all stimulus types inclusive null events have equal frequencies or $H_{0}$ : frequencies are equal to $A$-optimal frequencies in Liu \& Frank (2004) (unequal frequencies).

(a) Mean block lengths and stimulus frequencies of maximin design for estimation efficiency with $A$-optimality criterion

\begin{tabular}{cccc}
\hline Stimulus & Mean block length & Frequency & Theoretical \\
\hline 0 & $3.31 \mathrm{~s}$ & 0.34 & 0.37 \\
$\mathrm{~A}$ & $2.90 \mathrm{~s}$ & 0.23 & 0.21 \\
B & $2.95 \mathrm{~s}$ & 0.22 & 0.21 \\
C & $2.82 \mathrm{~s}$ & 0.22 & 0.21
\end{tabular}

Chi-square test (equal frequencies): $p=0.015$

Chi-square test (unequal frequencies): $p=0.797$

(b) Mean block lengths and stimulus frequencies of maximin design for estimation efficiency with $D$-optimality criterion

\begin{tabular}{cccc}
\hline Stimulus & Mean block length & Frequency & Theoretical \\
\hline 0 & $2.53 \mathrm{~s}$ & 0.24 & 0.25 \\
$\mathrm{~A}$ & $2.63 \mathrm{~s}$ & 0.25 & 0.25 \\
$\mathrm{~B}$ & $2.65 \mathrm{~s}$ & 0.25 & 0.25 \\
$\mathrm{C}$ & $2.65 \mathrm{~s}$ & 0.25 & 0.25 \\
\hline \multicolumn{2}{l}{ Chi-square test (equal frequencies) $: p=0.991$} \\
\hline
\end{tabular}

\subsubsection{Simulation III+IV: Multi-objective design criteria}

The multi-objective design criteria (MO-criteria) for both weighting schemes and both optimality criteria were increasing with increasing autocorrelation which can be explained by the interplay of standardized detection power and standardized estimation efficiency which in turn depend on the unstandardized design criterion and maximum design criterion. Furthermore, the minimum relative efficiencies of the locally optimal designs were in all simulations higher than 0.94 .

The maximin designs were obtained for intermediate values of $\rho(0.28-0.32)$ and had high minimum relative efficiencies (above 0.98). All maximin designs resembled more an event-related design than a blocked design because mean block lengths ranged from $3.24 \mathrm{~s}$ - $4.34 \mathrm{~s}$ (Table 3.4). This is partly due to the fact that randomized event-related designs perform better for detection power than blocked designs perform for estimation efficiency. This was seen by considering the detection power of $A$-optimal event-related designs for estimation efficiency (Simulation II) and the estimation efficiency of $A$-optimal blocked designs for detection power (Simulation Ia). Similar conclusions can be drawn from Birn et al. (2002, Figures 3 and 5) and Kao et al. (2009, Figure 2(A)). Furthermore, an event-related design scores higher for the counterbalancing criterion $F_{c}$ and the estimation efficiency $F_{e}$ whereas a blocked design scores only higher on $F_{d}$. The criterion $F_{f}$ for desired stimulus 
Table 3.4: Maximin designs with their mean block lengths, stimulus frequencies and theoretically optimal stimulus frequencies for both MO-criteria and both optimality criteria

(a) Mean block lengths and stimulus frequencies of maximin design for MO-criterion with equal weights for detection power and estimation efficiency and with $A$-optimality criterion

\begin{tabular}{cccc}
\hline Stimulus & Mean block length & Frequency & Theoretical \\
\hline 0 & $4.34 \mathrm{~s}$ & 0.35 & 0.37 \\
$\mathrm{~A}$ & $3.72 \mathrm{~s}$ & 0.21 & 0.21 \\
$\mathrm{~B}$ & $3.67 \mathrm{~s}$ & 0.22 & 0.21 \\
$\mathrm{C}$ & $3.80 \mathrm{~s}$ & 0.22 & 0.21 \\
\hline
\end{tabular}

(b) Mean block lengths and stimulus frequencies of maximin design for MO-criterion with equal weights for detection power and estimation efficiency and with $D$-optimality criterion

\begin{tabular}{cccc}
\hline Stimulus & Mean block length & Frequency & Theoretical \\
\hline 0 & $4.00 \mathrm{~s}$ & 0.24 & 0.25 \\
$\mathrm{~A}$ & $4.19 \mathrm{~s}$ & 0.25 & 0.25 \\
$\mathrm{~B}$ & $4.13 \mathrm{~s}$ & 0.25 & 0.25 \\
$\mathrm{C}$ & $4.13 \mathrm{~s}$ & 0.25 & 0.25 \\
\hline
\end{tabular}

(c) Mean block lengths and stimulus frequencies of maximin design for MO-criterion with equal weights on all four design criteria and with A-optimality criterion

\begin{tabular}{cccc}
\hline Stimulus & Mean block length & Frequency & Theoretical \\
\hline 0 & $4.24 \mathrm{~s}$ & 0.35 & 0.37 \\
$\mathrm{~A}$ & $3.39 \mathrm{~s}$ & 0.22 & 0.21 \\
$\mathrm{~B}$ & $3.24 \mathrm{~s}$ & 0.22 & 0.21 \\
$\mathrm{C}$ & $3.24 \mathrm{~s}$ & 0.22 & 0.21 \\
\hline
\end{tabular}

(d) Mean block lengths and stimulus frequencies of maximin design for MO-criterion with equal weights on all four design criteria and with $D$-optimality criterion

\begin{tabular}{cccc}
\hline Stimulus & Mean block length & Frequency & Theoretical \\
\hline 0 & $3.43 \mathrm{~s}$ & 0.24 & 0.25 \\
$\mathrm{~A}$ & $3.25 \mathrm{~s}$ & 0.25 & 0.25 \\
B & $3.33 \mathrm{~s}$ & 0.25 & 0.25 \\
C & $3.25 \mathrm{~s}$ & 0.25 & 0.25 \\
\hline
\end{tabular}


frequency is unaffected by the specific character of the design and can be equally high for event-related designs as well as for blocked designs. When comparing Table 3.4a with Table 3.4c and further Table 3.4b with Table 3.4d the mean block lengths are slightly lower for the MO-criterion with equal weights for all design criteria. The reason is probably that event-related designs score higher than blocked designs on $F_{c}$.

\subsubsection{Comparison of $A$ - and $D$-optimality criterion}

Figures $3.4 \mathrm{a}$ and $3.4 \mathrm{~b}$ show that the maximin designs perform best for the criterion by which they were obtained and that the relative efficiencies are in all comparisons above 0.86. It is shown in Figure 3.4a that for detection power of individual stimulus effects the relative efficiency for $\mathrm{MMD}_{A}$ vs. $\mathrm{MMD}_{D}$ is generally a little higher than the relative efficiency for $\mathrm{MMD}_{D}$ vs $\mathrm{MMD}_{A}$. As a conclusion, the $\mathrm{MMD}_{A}$ design is slightly more robust against the choice of optimality criterion than the $\mathrm{MMD}_{D}$ design when detection power of stimulus effects is considered. For detection of contrasts, the same maximin design MMD was obtained so that no comparison of maximin designs was necessary.

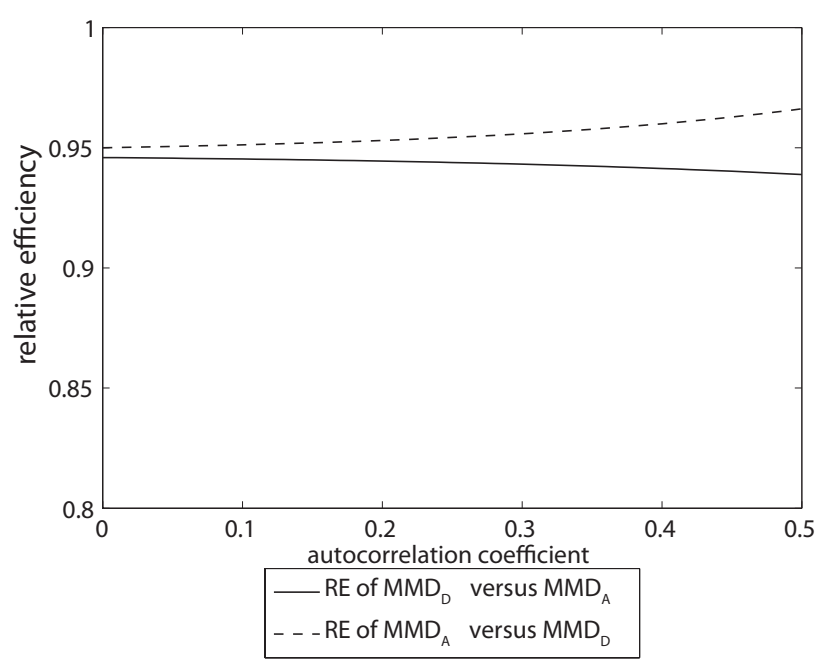

(a) Comparison of maximin designs for detection of individual stimuli by relative efficiency

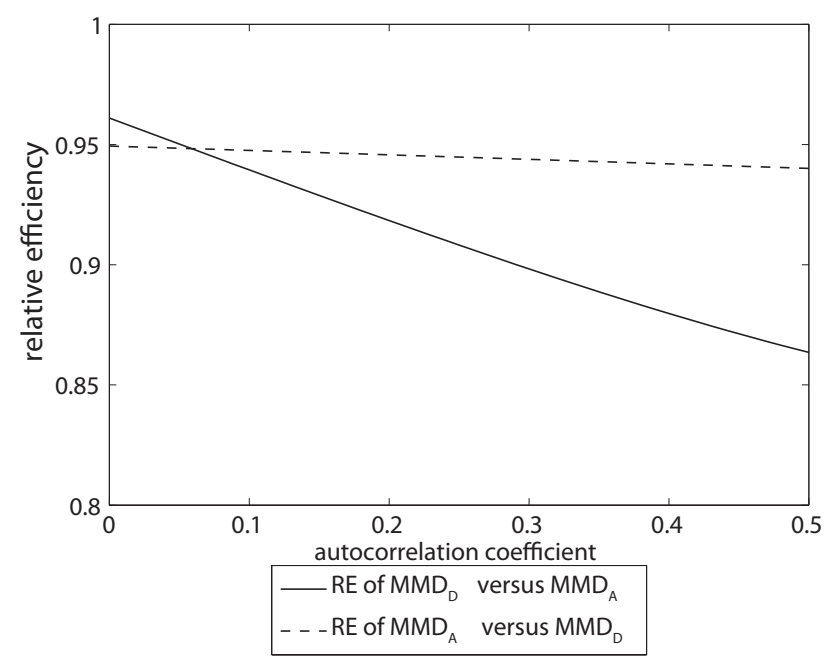

(b) Comparison of maximin designs for estimation efficiency by relative efficiency

Figure 3.4: Relative efficiencies for comparison of maximin designs for $A$-optimality criterion with maximin designs for $D$-optimality criterion. The relative efficiency of $\mathrm{MMD}_{D}$ versus $\mathrm{MMD}_{A}$ based on $A$-optimality and the relative efficiency of $\mathrm{MMD}_{A}$ versus $\mathrm{MMD}_{D}$ based on $D$-optimality is shown.

Figure $3.4 \mathrm{~b}$ shows that for estimation efficiency the relative efficiency for $\mathrm{MMD}_{D}$ versus $\mathrm{MMD}_{A}$ and the relative efficiency for $\mathrm{MMD}_{A}$ versus $\mathrm{MMD}_{D}$ both decrease as the autocorrelation increases. The relative efficiency of $\mathrm{MMD}_{D}$ versus $\mathrm{MMD}_{A}$ decreases to circa 0.86 whereas the relative efficiency of $\mathrm{MMD}_{A}$ versus $\mathrm{MMD}_{D}$ remains above 0.94. Thus, the maximin design $\mathrm{MMD}_{A}$ is more robust than $\mathrm{MMD}_{D}$ for estimation efficiency. 


\subsection{Discussion and conclusions}

Our main finding is that all maximin designs had a high minimum relative efficiency above 0.9 and were therefore very robust against misspecification of the autocorrelation. Moreover almost all locally optimal designs had a high minimum relative efficiency. Another important result is that the $A$ - and $D$-optimality criterion result in different optimal stimulus frequencies.

The maximin designs for detection of individual stimuli had the form of a blocked design with null blocks for both optimality criteria and their minimum relative efficiencies were 0.97 and 0.98 for the $A$-optimality criterion and the $D$-optimality criterion, respectively. Thus, these maximin designs were very efficient for the whole range of autocorrelation values. For detection of contrasts, the maximin designs had the form of a blocked design without null blocks and their minimum relative efficiencies were above 0.98. Furthermore, for almost all locally optimal designs the minimum relative efficiencies were above 0.9 and therefore quite high.

The maximin designs for estimation efficiency were event-related designs. For the $A$ optimality criterion, the maximin design had a minimum relative efficiency of 0.97 and all locally optimal designs had minimum relative efficiencies above 0.89 . For the $D$-optimality criterion, the minimum relative efficiencies were constantly high for all locally $D$-optimal designs. The maximin design $\mathrm{MMD}_{D}$ had a minimum relative efficiency of almost 1 . For the MO-criteria the minimum relative efficiencies of the maximin designs were above 0.98 . To conclude, in all cases the maximin designs and most locally optimal designs were robust against misspecification of the autocorrelation.

The $\mathrm{MMD}_{A}$ seemed to be slightly more robust against choice of optimality criterion than the $\mathrm{MMD}_{D}$ especially for estimation efficiency as can be seen in Figure 3.4. For detection power both designs $\mathrm{MMD}_{A}$ and $\mathrm{MMD}_{D}$ are robust against choice of optimality criterion. General advantages of the $D$-optimality criterion are that the $D$-optimal design is scale independent and results in a minimized confidence ellipsoid for the unknown parameters or contrasts of parameters. Based on our results for $Q=3$, we recommend using the $A$-optimality criterion for estimation efficiency. For detection power, we recommend the use of the $D$-optimality criterion. More research for other number of stimuli is however needed.

One important difference between $A$ - and $D$-optimal designs was found. For all simulations except detection power of contrasts (simulation Ib) it was seen that the $D$-optimality criterion resulted in a maximin design with equal frequencies for all stimulus types including null events whereas the $A$-optimality criterion resulted in a maximin design with equal frequencies for stimulus events and a higher frequency for null events. The frequencies for the $A$-optimality criterion were in agreement with the approximated optimal stimulus frequencies of Liu \& Frank (2004) which are also based on the $A$-optimality criterion. We derived the optimal frequency for the $D$-optimality criterion and found that stimulus frequencies obtained by the genetic algorithm were close to these frequencies. Furthermore, the mean block length for null events was higher than the mean block length for stimulus types when the $A$-optimality criterion was applied for detection power of individual stimuli.

The different behavior of the $A$ - and $D$-optimality criterion with respect to stimulus fre- 
quency has interesting consequences for the performance of $m$-sequences which are known to be very efficient for estimation efficiency (Buračas \& Boynton, 2002). Kao et al. (2009) found that $m$-sequences were inferior to their $A$-optimal designs obtained by the GA for estimation efficiency even when no $m$-sequences were included into the starting designs. One reason for this might be that $m$-sequences will generate sequences with equal probability for all stimulus types including null events which is not $A$-optimal. However, we showed that equal frequencies for all stimulus types including null events is $D$-optimal. So, the degree of inferiority of $m$-sequences might actually depend on the optimality criterion, i.e., $A$ - or $D$-optimality criterion.

Another interesting result of our simulations is that detection power decreases with an increase of autocorrelation whereas estimation efficiency increases. This was the case for both optimality criteria. A similar result was found by Birn et al. (2002) where detection power became smaller when correlated noise instead of uncorrelated noise was applied whereas estimation efficiency increased for correlated noise in comparison with uncorrelated noise. An explanation for these effects could be an interplay of the following three aspects: the power spectrum of the hemodynamic response function, power spectrum of the noise structure and the power spectrum of the design paradigma.

Event-related designs will have a power spectrum with peaks at all frequencies similar to uncorrelated error/white noise (Henson, 2007). We plotted the power spectrum for some of the locally optimal designs for estimation efficiency and saw that indeed all frequencies were represented. In contrast, blocked designs will have a peak at the frequency which corresponds to the alternation frequency, e.g., $0.02 \mathrm{~Hz}$ if the sum of the mean block lengths, including null events, is 50 seconds. Furthermore, peaks will be at multiples of the frequency $0.02 \mathrm{~Hz}$. With increasing autocorrelation, AR1 noise becomes more like low frequency noise. Since the $A$ - and $D$-optimal event-related designs have all frequencies represented in their power spectrum, they might be positively affected by the increasing autocorrelation. In contrast, $A$ - and $D$-optimal blocked designs which have dominantly low frequencies represented in their power spectrum might be negatively affected.

The block lengths of the maximin designs for the $A$ - as well as the $D$-optimality criterion for detection are not in agreement with the recommendations which can be found in literature where the optimal block length is given as $14 \mathrm{~s}$ to $20 \mathrm{~s}$ (Skudlarski et al., 1999; Aguirre \& D'Esposito, 1999; Chein \& Schneider, 2003; Wüstenberg et al., 2005). However, it has to be taken into account that some of these recommendations were given for simple designs with two alternating conditions and that no differentiation between length of null blocks and stimulus blocks was considered in the literature. One reason for the difference between our optimal block length and the literature might be that the optimal block length depends on the number of stimulus types. We considered several simulations with varying number of stimulus types but could not discover a clear relationship between the number of stimulus types and optimal block lengths.

An explanation for the optimal block lengths in Tables 3.1 and 3.2 is based on the effect of the autocorrelation. Applying low frequency noise in the form of AR1 seems to increase optimal design frequency and thus shorter block lengths are favored more than for uncorrelated noise. The higher the autocorrelation the shorter block lengths and the 
higher the design frequency seems to be. The same effect was found by Wager \& Nichols (2003) for detection of contrasts. Considering the locally optimal designs for detection we could indeed see that block lengths were decreasing with increasing autocorrelation for both the $A$ - and the $D$-optimality criterion. This effect arose for detection of individual stimulus effects as well as for contrasts. As the maximin design is chosen to be efficient for all autocorrelation values, shorter block lengths than in literature can be explained by this effect of autocorrelation.

It should be noted that our simulation results were obtained for three stimulus types and the question remains whether the same results would be obtained for other numbers of stimulus types. Based on preliminary results and the results of Kao et al. (2009), we infer that the obtained stimulus frequencies would be similar to the theoretically obtained stimulus frequencies. Further research is however needed to draw firmer conclusions but is beyond the scope of this paper.

The presented methodology using the maximin criterion can also be applied to vary the parameters of the canonical hemodynamic response function and examine the robustness of the optimal design against misspecification of the specific HRF shape. To apply the genetic algorithm without maximin criterion, we would recommend fixing the autocorrelation $\rho$ to an intermediate value. The minimum relative efficiencies of most locally optimal designs were high so that the specific value of $\rho$ is less important as long as a realistic value of $\rho$ is chosen. Robust designs can be obtained in this way via the genetic algorithm.

\section{A Derivation of optimal stimulus frequency for $D$ - optimality criterion}

\section{A.1 Useful formulae}

We are going to apply the Kronecker product (Harville, 2008) and its following properties

$$
\begin{aligned}
(A \otimes B)^{-1} & =A^{-1} \otimes B^{-1} \\
(A \otimes B)^{T} & =A^{T} \otimes B^{T} \\
(A \otimes B)(C \otimes D) & =A C \otimes B D \\
\operatorname{det}(A \otimes B) & =\operatorname{det}(A)^{\operatorname{rank}(B)} \cdot \operatorname{det}(B)^{\operatorname{rank}(A)} .
\end{aligned}
$$

Another useful formula will be:

$$
\operatorname{det}\left(A+u v^{T}\right)=\left(1+v^{T} A^{-1} u\right) \operatorname{det}(A) .
$$

\section{A.2 Approximation of Fisher information matrix}

The GLS estimator for $h$ in Eq. (3.1) is equal to

$$
\hat{h}=\left(X_{\perp}^{T} X_{\perp}\right)^{-1} X_{\perp}^{T} V Y
$$

where $X_{\perp}=\left(I-P_{V S}\right) V X$ and the Fisher information matrix is given by $X_{\perp}^{T} X_{\perp}$. 
The Fisher information matrix consists of block diagonal terms of the form $X_{\perp, q}^{T} X_{\perp, q}$ where $X_{\perp, q}$ is the design matrix of the $q$ th trial type and the $q$ th submatrix of $X_{\perp}$. The block off-diagonal terms are given by $X_{\perp, q}^{T} X_{\perp, r}$. Liu \& Frank (2004) approximated the Fisher information matrix by the following expression:

$$
X_{\perp}^{T} X_{\perp} \approx E_{Q} \otimes A_{k}
$$

where the $k \times k$ matrix $A_{k}$ is the average autocorrelation matrix $A_{k} \approx(G / Q) \sum_{q=1}^{Q} X_{\perp, q}^{T} X_{\perp, q}$ and $G$ is a scaling factor. The $Q \times Q$ matrix $E_{Q}$ consists of the $\rho(q, r)$ crosscorrelation terms between the whitened and detrended stimulus patterns of trial type $q$ and trial type $r$.

Liu \& Frank (2004) showed that the matrix $E_{Q}$ has the following expression under the assumption of uncorrelated noise and $S$ being a vector of ones:

$$
E_{Q}=N p\left(I_{Q}-p 1_{Q} 1_{Q}^{T}\right)
$$

with $p$ being the stimulus frequency which was equal for all stimulus types and $1_{Q}$ being a $(Q \times 1)$ column vector with all elements equal to 1 . Further, $Q$ is the number of stimulus types and $N$ is the number of time points. From Eq. (3.17) it follows that $\operatorname{det}\left(E_{Q}\right)=$ $(N p)^{Q}(1-p Q)$. Finally, Liu \& Frank (2004) present an expression for the inverse $E_{Q}^{-1}$.

\section{A.3 D-optimal stimulus frequency for estimation efficiency and detection power of individual stimulus effects}

We will show that estimation efficiency with the purpose of estimating the individual HRFs and detection power with the purpose of estimating individual stimulus effects is optimized with a stimulus frequency of $p=1 /(Q+1)$ under the given assumptions and the $D$-optimality criterion.

To find the optimal stimulus frequency for efficient estimation of $h$ in Eq. (3.1), we have to minimize the following expression

$$
\begin{aligned}
\operatorname{det}\left(\left(X_{\perp}^{T} X_{\perp}\right)^{-1}\right) & =\operatorname{det}\left(E_{Q}^{-1} \otimes A_{k}^{-1}\right) \\
& =\operatorname{det}\left(E_{Q}^{-1}\right)^{\operatorname{rank}\left(A_{k}\right)} \cdot \operatorname{det}\left(A_{k}^{-1}\right)^{\operatorname{rank}\left(E_{Q}\right)} \\
& =\operatorname{det}\left(E_{Q}^{-1}\right)^{k} \cdot \operatorname{det}\left(A_{k}^{-1}\right)^{Q} \\
& =\left(\frac{1}{(N p)^{Q}(1-p Q)}\right)^{k} \cdot \operatorname{det}\left(A_{k}^{-1}\right)^{Q} \\
& =\frac{1}{N^{Q k}} \cdot f(p, Q)^{k} \cdot \operatorname{det}\left(A_{k}^{-1}\right)^{Q},
\end{aligned}
$$

where $f(p, Q)=\left(p^{Q}(1-p Q)\right)^{-1}$. The factors $Q$ and $N$ are assumed to be fixed and $f(p, Q)$ is minimized for $p=1 /(Q+1)$.

The optimal stimulus frequency for detection of individual stimulus effects is obtained by minimizing

$$
\operatorname{det}\left(\left(Z_{\perp}^{T} Z_{\perp}\right)^{-1}\right),
$$


where $Z_{\perp}=\left(I-P_{V S}\right) V Z$ is the whitened and detrended design matrix. Following Liu \& Frank (2004) we can write $Z_{\perp}=\left[\begin{array}{llll}X_{\perp, 1} \bar{h}_{1} & X_{\perp, 2} \bar{h}_{2} & \ldots & X_{\perp, Q} \bar{h}_{Q}\end{array}\right]$, where $\bar{h}_{i}$ are vectors giving the assumed hemodynamic response shape for trial type $i$. We assume that there is only one hemodynamic response shape for all trial types and replace $\bar{h}_{i}$ with $\bar{h}_{0}$. Thus, $Z_{\perp}=\left[\begin{array}{llll}X_{\perp, 1} \bar{h}_{0} & X_{\perp, 2} \bar{h}_{0} & \ldots & X_{\perp, Q} \bar{h}_{0}\end{array}\right]=X_{\perp}\left(I_{Q} \otimes \bar{h}_{0}\right)$. We see using Eqs. (3.14) and (3.15) that

$$
\begin{aligned}
Z_{\perp}^{T} Z_{\perp} & =\left(X_{\perp}\left(I_{Q} \otimes \bar{h}_{0}\right)\right)^{T} X_{\perp}\left(I_{Q} \otimes \bar{h}_{0}\right) \\
& =E_{Q} \otimes \bar{h}_{0}^{T} A_{k} \bar{h}_{0},
\end{aligned}
$$

where $\bar{h}_{0}^{T} A_{k} \bar{h}_{0}$ is a scalar. Therefore

$$
\begin{aligned}
\operatorname{det}\left(Z_{\perp}^{T} Z_{\perp}\right)^{-1} & =\operatorname{det}\left(E_{Q} \cdot \bar{h}_{0}^{T} A_{k} \bar{h}_{0}\right)^{-1} \\
& =\left(\bar{h}_{0}^{T} A_{k} \bar{h}_{0}\right)^{-Q} \cdot \operatorname{det}\left(E_{Q}^{-1}\right) \\
& =\left(\bar{h}_{0}^{T} A_{k} \bar{h}_{0}\right)^{-Q} \cdot \frac{1}{N^{Q}} \cdot f(p, Q),
\end{aligned}
$$

with $f(p, Q)=\left(p^{Q}(1-p Q)\right)^{-1}$. As mentioned previously, $f(p, Q)$ is minimized for $p=$ $1 /(Q+1)$.

\section{A.4 D-optimal stimulus frequency for estimation efficiency of contrasts and for detection power of contrasts}

For estimation efficiency, we are interested in finding the optimal stimulus frequency to estimate the pairwise contrasts between individual HRFs (Kao et al., 2007). We will focus here on possible $(Q-1)$ linear independent pairwise contrasts between $Q$ HRFs of $k$ time points.

In 3.A.5 we show that this frequency is independent of the contrast matrix $C$. Now we will assume that $C^{T}$ is of the form

$$
C^{T}=\left(\begin{array}{cccccc}
1 & -1 & 0 & \ldots & \ldots & 0 \\
1 & 0 & -1 & 0 & \ldots & 0 \\
\vdots & \vdots & 0 & \ddots & \ddots & \vdots \\
\vdots & \vdots & \vdots & \ldots & \ddots & 0 \\
1 & 0 & \ldots & \ldots & 0 & -1
\end{array}\right)
$$

The $(Q-1) \times Q$ matrix $C^{T}$ contains $Q-1$ linear independent contrasts which is the maximum of possible linear independent contrasts.

For estimation of contrasts of individual HRFs we want to minimize

$$
\operatorname{det}\left(\left(C^{T} \otimes I_{k}\right)\left(X_{\perp}^{T} X_{\perp}\right)^{-1}\left(C^{T} \otimes I_{k}\right)^{T}\right)=\operatorname{det}\left(C^{T} E_{Q}^{-1} C\right)^{k} \operatorname{det}\left(A_{k}^{-1}\right)^{Q} .
$$


The following equation holds

$$
\begin{aligned}
& C^{T} E_{Q}^{-1} C \\
= & \frac{1}{p N(1-Q p)}\left(\begin{array}{ccccc}
2(1-Q p) & 1-Q p & \ldots & \ldots & 1-Q p \\
1-Q p & 2(1-Q p) & 1-Q p & \ldots & \vdots \\
\vdots & \ddots & \ddots & \ddots & \vdots \\
\vdots & \vdots & \ddots & \ddots & 1-Q p \\
1-Q p & \ldots & \ldots & 1-Q p & 2(1-Q p)
\end{array}\right) \\
= & \frac{1}{p N(1-Q p)}\left((1-p Q) I_{Q-1}+(1-p Q) 1_{Q-1} 1_{Q-1}^{T}\right) .
\end{aligned}
$$

Using Eq. (3.17), the determinant is therefore given by

$$
\begin{aligned}
\operatorname{det}\left(C^{T} E_{Q}^{-1} C\right) & =\left(\frac{1}{p N(1-Q p)}\right)^{Q-1} \cdot Q(1-p Q)^{Q-1} \\
& =\frac{Q}{N^{Q-1} p^{Q-1}}
\end{aligned}
$$

Thus,

$$
\begin{aligned}
\operatorname{det}\left(\left(C^{T} \otimes I_{k}\right)\left(X_{\perp}^{T} X_{\perp}\right)^{-1}\left(C^{T} \otimes I_{k}\right)^{T}\right) & =\frac{Q}{N^{Q-1} p^{Q-1}} \operatorname{det}\left(A_{k}^{-1}\right)^{Q} \\
& =\frac{Q}{N^{Q-1}}\left(f_{C}(p)\right)^{Q-1} \operatorname{det}\left(A_{k}^{-1}\right)^{Q}
\end{aligned}
$$

where $f_{C}(p)=1 / p$. As $p$ is the equal probability of all trial types, $p \leq 1 / Q$. So, $f_{C}$ is minimized if $p=1 / Q$. We have shown that for estimation efficiency the contrast between individual HRFs is optimized with the stimulus frequency of $p=1 / Q$ for the $D$-optimality criterion. To optimize detection power for contrasts between stimuli using the contrast matrix $C$ as given above, we have to minimize

$$
\begin{aligned}
\operatorname{det}\left(C^{T}\left(Z_{\perp}^{T} Z_{\perp}\right)^{-1} C\right) & =\operatorname{det}\left(C^{T}\left(E_{Q}^{-1} \cdot\left(h_{0}^{T} A_{k} h_{0}\right)^{-1}\right) C\right) \\
& =\left(h_{0}^{T} A_{k} h_{0}\right)^{-(Q-1)} \operatorname{det}\left(C^{T} E_{Q}^{-1} C\right) \\
& =\frac{Q}{N^{Q-1}}\left(f_{C}(p)\right)^{Q-1}\left(h_{0}^{T} A_{k} h_{0}\right)^{-(Q-1)}
\end{aligned}
$$

So, the detection of individual contrasts is also optimized by $p=1 / Q$.

\section{A.5 Proof: Independence of $D$-optimal stimulus frequency from contrast matrix}

For each contrast matrix $C_{1}$, there exists a $(Q-1) \times(Q-1)$ square matrix $B$ of full rank so that $C_{1}^{T}=B C^{T}$ as one given contrast in $C_{1}$ can be generated from the contrasts in $C$ by linear transformation. Therefore,

$$
\begin{aligned}
\operatorname{det}\left(C_{1}^{T} E_{Q}^{-1} C_{1}\right) & =\operatorname{det}\left(B C^{T} E_{Q}^{-1} C B^{T}\right) \\
& =\operatorname{det}(B) \operatorname{det}\left(C^{T} E_{Q}^{-1} C\right) \operatorname{det}\left(B^{T}\right)
\end{aligned}
$$


where $B$ only contains values of 0,1 and -1 . Furthermore, $\operatorname{det}(B)= \pm 1$ and therefore $\operatorname{det}\left(C_{1}^{T} E_{Q}^{-1} C_{1}\right)=\operatorname{det}\left(C^{T} E_{Q}^{-1} C\right)$ for all contrast matrices $C_{1}$. As $\operatorname{det}\left(C^{T} E_{Q}^{-1} C\right)$ was the relevant term for detection power and estimation efficiency to calculate $f_{C}(p)$, it shows that the $D$-optimal stimulus frequency is independent of the contrast matrix. 


\section{CHAPTER 4}

\section{Optimal design for nonlinear estimation of the hemodynamic response function}

This chapter will be published in Human Brain Mapping (article in press) with co-authors Gerard J. P. van Breukelen, Rainer Goebel and Martijn P. F. Berger. 


\begin{abstract}
Subject-specific hemodynamic response functions (HRFs) have been recommended to capture variation in the form of the hemodynamic response between subjects (Aguirre et al., 1998). The purpose of this paper is to find optimal designs for estimation of subject-specific parameters for the double gamma HRF. As the double gamma function is a nonlinear function of its parameters, optimal design theory for nonlinear models is employed in this paper (Atkinson et al., 2007). The double gamma function is linearized by a Taylor approximation and the maximin criterion is used to handle dependency of the $D$-optimal design on the expansion point of the Taylor approximation. A realistic range of double gamma HRF parameters is used for the expansion point of the Taylor approximation. Furthermore, a genetic algorithm (GA) (Wager \& Nichols, 2003; Kao et al., 2009) is applied to find locally optimal designs for the different expansion points and the maximin design chosen from the locally optimal designs is compared to maximin designs obtained by $m$-sequences, blocked designs, designs with constant interstimulus interval (ISI) and random event-related designs. The maximin design obtained by the GA is most efficient. Random event-related designs chosen from several generated designs and $m$-sequences have a high efficiency, while blocked designs and designs with a constant ISI have a low efficiency compared to the maximin GA design.
\end{abstract}

Keywords: fMRI, nonlinear model, double gamma function, subject-specific HRF, genetic algorithm, optimal design 


\subsection{Introduction}

The hemodynamic response function (HRF) describes the functional magnetic resonance imaging (fMRI) signal change to one short stimulus based on the blood-oxygen-leveldependent contrast (Logothetis \& Wandell, 2004). Most statistical analyses of fMRI experiments are based on a general linear model where one specific shape for the HRF for all subjects and voxels is assumed, e.g., the canonical double gamma function with fixed parameter values (Friston et al., 1998). However, it is well established that the HRF varies per subject (Aguirre et al., 1998; Boynton et al., 1996; Zarahn et al., 1997) and brain region (Miezin et al., 2000; Handwerker et al., 2004; Zarahn et al., 1997). For this reason, Aguirre et al. (1998) suggested to handle the variability of the hemodynamic response function by applying subject-specific hemodynamic response functions. Region-specific functions might also be justified but in general the variability has been found to be more expressed between subjects than between regions in one subject (Handwerker et al., 2004). Using a more accurate model for the hemodynamic response function by allowing variability between subjects increases sensitivity (Aguirre et al., 1998). In the following we will focus on subject-specific HRFs, while our methods and results can equally be applied to determine optimal designs for nonlinear estimation of region-specific HRF parameters.

Handwerker et al. (2004) compared the use of a subject-specific HRF to a canonical HRF with regard to false negatives, t-values and misestimation of the amplitude of the HRF. They showed that the subject-specific HRF performed better than the canonical HRF. Although differences between subject-specific and canonical HRF were small, they were statistically significant and will especially be relevant for cross-subject analyses. Acknowledging the relevance and importance of using subject-specific HRFs, the optimal design for estimation of such a HRF has to be found. The purpose of this paper is to find the optimal design for nonlinear estimation of the parameters of the double gamma function which is a common model for the HRF and provides a good fit to the general form of the HRF.

A first experiment with one stimulus type can be performed to estimate the subjectspecific hemodynamic response function independently of subsequent experiments. From this first experiment, activated voxels or brain regions can be identified by fitting a general linear model based on a Fourier basis set or finite impulse response (FIR) basis set and corrected for multiple comparisons and dependence across voxels (Handwerker et al., 2004). Furthermore, the hemodynamic response function can be estimated nonlinearly from the identified voxels or brain regions. The subject-specific hemodynamic response function can then be employed in fMRI data analyses for subsequent experiments with the same subject and more stimulus types, as variability across scan sessions has been proven to be minor (Aguirre et al., 1998). Despite the fact that additional scanning time is necessary to firstly derive a subject-specific HRF, Handwerker et al. (2004) showed that if the fit of the canonical HRF is poor a subject-specific HRF will be beneficial.

Besides using subject-specific HRF, several other methods to capture variability of the HRF have been applied. Fourier basis sets (Josephs et al., 1997), splines (Crellin et al., 1999) and finite impulse response sets (Ollinger et al., 2001) are flexible in modeling the shape of the hemodynamic response, but are less parsimonious and provide less powerful 
tests than a double gamma function (Henson \& Friston, 2007). The interpretation of the parameters of these sets is also less direct than with a derived subject-specific double gamma function where only one parameter reflecting the amplitude of the hemodynamic response function has to be estimated. Goutte et al. (2000) extended the approach of HRF estimation based on finite impulse response sets by assuming a Gaussian prior on the finite impulse response coefficients. This forces the HRF to be smooth and reduces the risk of ill determined parameters and overfitted models (Goutte et al., 2000).

Another approach to handle variability of the hemodynamic response from the standard form is the use of the canonical hemodynamic response function plus partial derivatives (Friston et al., 1998). The partial derivatives with respect to time and dispersion can capture small derivations from the canonical form. However, the amount of deviations that can be captured are small, i.e., up to $1 \mathrm{~s}$ in peak latency (Lindquist et al., 2009). Subject-specific hemodynamic response functions could capture more variability. Lindquist \& Wager (2007) proposed a HRF model based on the superposition of three inverse logit functions (IL) and showed that parameter estimates of response height, delay and width are more independent of each other by using their model instead of other common HRF models. Furthermore, deconvolution analysis, e.g., via a Wiener filter (Glover, 1999), can be used to estimate the hemodynamic response function but deconvolution analysis is a noisy process and the deconvolution parameters must be chosen carefully (Glover, 1999). Apart from the HRFs models mentioned so far, several other models (Rajapakse et al., 1998; Woolrich et al., 2004; Buxton et al., 2004; Ciuciu et al., 2003) have been proposed with their own advantages and disadvantages. Further details of other HRFs models, comparisons between models and the relevance of precise HRF estimation can be found in Lindquist \& Wager (2007). The focus in this paper is on using subject-specific parameters for the double gamma HRF which can be estimated from a nonlinear model by a nonlinear iterative least squares fitting algorithm, e.g., the Levenberg-Marquardt algorithm which uses a first-order Taylor approximation (Miezin et al., 2000; Handwerker et al., 2004; Lindquist \& Wager, 2007).

Optimal design for estimation of the HRF by means of a nonlinear model has not been considered yet. Previous research on optimal design for fMRI experiments has focused on optimizing detection power and estimation efficiency (Dale, 1999; Liu et al., 2001; Buračas \& Boynton, 2002; Birn et al., 2002). Detection power assumes a fixed shape of the HRF with known HRF parameters and refers to the power of a design to detect task-specific activation of brain regions whereas estimation efficiency refers to the efficiency of a design to estimate the hemodynamic response function based on a linear model with a binary design matrix indicating stimulus presentations at certain time points (Dale, 1999; Henson \& Friston, 2007; Liu et al., 2001). Several designs, e.g., $m$-sequences (Buračas \& Boynton, 2002), random event-related designs (Friston et al., 1999; Hagberg et al., 2001), blocked designs, and optimal designs generated by genetic algorithms (Wager \& Nichols, 2003; Kao et al., 2009) have been studied and applied to increase detection power and estimation efficiency. In this paper, these commonly used designs are applied to find an optimal design for nonlinear estimation of the double gamma function parameters, and a thorough and balanced comparison of the efficiency of these various designs is provided. By means of the 
genetic algorithm (GA), optimal designs can be obtained without previous specialization of the design properties. Because the design space of all possible fMRI designs is enormous (Buračas \& Boynton, 2002), this algorithm is useful to provide an efficient search within the design space (Kao et al., 2009). It is known that blocked designs are optimal for detection power, whereas rapid event-related designs with randomized interstimulus intervals are optimal for estimation efficiency based on linear models (Liu \& Frank, 2004; Liu, 2004; Birn et al., 2002). However, not any randomly generated rapid event-related design achieves high estimation efficiency (Buračas \& Boynton, 2002), and the genetic algorithm provides more efficient designs than a random search in the design space (Wager \& Nichols, 2003). Our results show that a mixture of a blocked design and a rapid event-related design will be most efficient for the nonlinear model but that rapid event-related designs can also be very efficient for the nonlinear model.

To calculate the efficiency of a given design, e.g., an $m$-sequence, a Taylor approximation of first order is applied to linearize the model with respect to the unknown parameters of the HRF (Atkinson et al., 2007). Based on the linearized model, the covariance matrix of the generalized least squares (GLS) estimator can be calculated for a given expansion point of the Taylor approximation. The $D$-optimality criterion is then considered to determine the optimal design which minimizes the determinant of the covariance matrix of the GLS estimators for the linearized model. The determinant of the GLS estimator is proportional to the area of the confidence ellipsoid for the estimator. Thus, minimizing the determinant leads to a minimized confidence ellipsoid. However, for the Taylor approximation an expansion point is needed. The optimal design may then depend on the expansion point and is thus locally optimal. To handle local optimality, a range for HRF parameters describing commonly observed shapes of the HRF was chosen from literature (Aguirre et al., 1998; Le et al., 2001; de Zwaart et al., 2005; Handwerker et al., 2004; Glover, 1999) and a maximin approach (Atkinson et al., 2007) was applied. The $D$-optimality criterion and the maximin criterion will be explained in Section 4.2.

\subsection{Theory}

The fMRI signal $Y$ to one stimulus type can be described by the following model

$$
Y=Z \beta+S \gamma+\epsilon=X h(\theta) \beta+S \gamma+\epsilon
$$

where $Z$ is an $T \times 1$ vector, $\beta$ is the amplitude of the hemodynamic response, $S$ is a nuisance matrix modeling, e.g., scanner drift, and $\gamma$ is the vector with unknown nuisance parameters. We assume the error $\epsilon$ to be normally distributed with expectation 0 and covariance matrix $\Sigma$. The number of time points for the fMRI experiment is given by $T$. The design vector $Z$ is obtained by multiplication of the zero-one $T \times k$ matrix $X$ with the assumed hemodynamic response vector $h(\theta)=(h(1, \theta), \ldots, h(k, \theta))^{T}$, where $k$ is the number of time points for the HRF and $\theta$ is the assumed parameter vector for the HRF. The matrix $X$ is the stimulus convolution matrix, and the product $X h(\theta)$ equals the discrete convolution of the stimulus sequence, which indicates an occurrence of a stimulus at a certain time point, with the hemodynamic response function $h$ for parameter $\theta$ (Dale, 
1999). To estimate the hemodynamic response function, $Y$ will here be the spatially averaged time course from a set of activated voxels, i.e., a region of interest (ROI).

The vector $h(\theta)$ depends on the parameters for the hemodynamic response function. A commonly employed function is the double gamma function with parameter vector $\theta=$ $\left(a_{1}, b_{1}, c, a_{2}, b_{2}\right)$ :

$$
h(t, \theta)=\frac{b_{1}^{a_{1}+1} t^{a_{1}} \exp \left(-b_{1} t\right)}{\Gamma\left(a_{1}+1\right)}-\frac{b_{2}^{a_{2}+1} t^{a_{2}} \exp \left(-b_{2} t\right)}{c \Gamma\left(a_{2}+1\right)},
$$

where $\Gamma$ is the Gamma function and $t$ is time in seconds since stimulus onset. The first gamma distribution in Eq. (4.2) models the peak of the HRF and the second gamma distribution in Eq. (4.2) models the undershoot of the HRF. For detection of activation by stimuli presentation, the main interest is normally in the peak of the HRF and its amplitude. However, more variation in the undershoot than in the peak has been reported and incorrect modeling of the undershoot might decrease statistical sensitivity (Handwerker et al., 2004). Thus, all parameters were included in the Taylor approximation to study optimal designs for simultaneous estimation of all double gamma parameters. First-order Taylor expansion of $h(t, \theta)$ with respect to $\theta$ and expansion point $\theta_{0}$ gives

$$
h(t, \theta) \approx h\left(t, \theta_{0}\right)+\left.\sum_{j=1}^{5} \frac{\partial h(t, \theta)}{\partial \theta_{j}}\right|_{\theta=\theta_{0}}\left(\theta_{j}-\theta_{0, j}\right) .
$$

where $\theta_{0, j}$ is the $j$ th entry in the expansion point $\theta_{0}$ and $\theta_{j}$ is the $j$ th entry in the point $\theta$. Equation (4.3) is a Taylor expansion of $h(t, \theta)$ with respect to the several variables in $\theta=\left(a_{1}, b_{1}, c, a_{2}, b_{2}\right)$. The partial derivatives of these variables are denoted by $\partial h(t, \theta) / \partial \theta_{j}$, e.g., $\partial h(t, \theta) / \partial \theta_{1}$ is the partial derivative with respect to $a_{1}$ etc. These partial derivatives with respect to $\theta_{j}$ are evaluated in Eq. (4.3) at the expansion point $\theta_{0}$ to give the slope of the tangent at $h(t, \theta)$ in point $\theta_{0}$ into the direction of $\theta_{j}$. These slopes are multiplied with the distance $\left(\theta_{j}-\theta_{0, j}\right)$ between the $j$ th entries of $\theta$ and $\theta_{0}$, and the sum of these products plus $h\left(t, \theta_{0}\right)$ gives an estimate of $h(t, \theta)$.

Substituting Eq. (4.3) in Eq. (4.1) we obtain

$$
Y \approx X H\left(\theta_{0}\right) \tau+S \gamma+\epsilon
$$

where the matrix $H\left(\theta_{0}\right)$ contains the hemodynamic response function (double gamma function) and its derivatives sampled at time points $1, \ldots, k$ and evaluated at expansion point $\theta_{0}$ :

$$
H\left(\theta_{0}\right)=\left(\begin{array}{cccc}
h\left(1, \theta_{0}\right) & \left.\frac{\partial h(1, \theta)}{\partial \theta_{1}}\right|_{\theta=\theta_{0}} & \ldots & \left.\frac{\partial h(1, \theta)}{\partial \theta_{5}}\right|_{\theta=\theta_{0}} \\
h\left(2, \theta_{0}\right) & \left.\frac{\partial(2, \theta)}{\partial \theta_{1}}\right|_{\theta=\theta_{0}} & \ldots & \left.\frac{\partial h(2, \theta)}{\partial \theta_{5}}\right|_{\theta=\theta_{0}} \\
\vdots & \vdots & & \vdots \\
h\left(k, \theta_{0}\right) & \left.\frac{\partial h(k, \theta)}{\partial \theta_{1}}\right|_{\theta=\theta_{0}} & \ldots & \left.\frac{\partial h(k, \theta)}{\partial \theta_{5}}\right|_{\theta=\theta_{0}}
\end{array}\right),
$$

and $\tau=\left(\beta, \beta\left(\theta_{1}-\theta_{0,1}\right), \ldots, \beta\left(\theta_{5}-\theta_{0,5}\right)\right)^{T}$. The first column of the matrix $H\left(\theta_{0}\right)$ contains the double gamma function $h$ sampled at time points $1, \ldots, k$, and evaluated at the HRF parameter vector $\theta_{0}$. The second column contains the partial derivative of $h$ with respect 
to $\theta_{1}$, thus $a_{1}$, evaluated at expansion point $\theta_{0}$ and sampled at time points $1, \ldots, k$. Analogously, the other columns consists of the partial derivatives with respect to $\theta_{2}\left(b_{1}\right), \theta_{3}(c)$, $\theta_{4}\left(a_{2}\right)$ and $\theta_{5}\left(b_{2}\right)$ sampled at time points $1, \ldots, k$ and evaluated at expansion $\theta_{0}$. Equation (4.4) can be rewritten as

$$
Y \approx Z \tau+S \gamma+\epsilon
$$

with $Z=X H\left(\theta_{0}\right)$. This illustrates that the model in Eq. (4.6) is similar to a model for detection of task-related activation with a HRF basis set consisting of the canonical double gamma function for parameter $\theta_{0}$ and the derivatives of the double gamma function with respect to $a_{1}, b_{1}, c, a_{2}$ and $b_{2}$ evaluated at $\theta_{0}$ (Henson \& Friston, 2007; Liu et al., 2001; Kao et al., 2009). However, the model in Eq. (4.6) is also related to a model for linear estimation of the HRF as the parameters of the double gamma function will be estimated from Eq. (4.6). The GLS estimator for $\left(\tau^{T}, \gamma^{T}\right)^{T}$ in Eq. (4.6) is given by

$$
\begin{aligned}
\left(\begin{array}{c}
\hat{\tau} \\
\hat{\gamma}
\end{array}\right) & =\left((Z S)^{T} \Sigma^{-1}(Z S)\right)^{-1}(Z S)^{T} \Sigma^{-1} Y \\
& =\left(\begin{array}{cc}
Z^{T} \Sigma^{-1} Z & Z^{T} \Sigma^{-1} S \\
S^{T} \Sigma^{-1} Z & S^{T} \Sigma^{-1} S
\end{array}\right)^{-1}\left(\begin{array}{c}
Z^{T} \\
S^{T}
\end{array}\right) Y
\end{aligned}
$$

From Eq. (4.7) using Theorem 8.5.11 in Harville (1997) for the inversion of partitioned matrices, it is seen that the GLS estimator of $\tau$ is given by

$$
\hat{\tau}=\left(Z^{T} V^{T}\left(I-P_{V S}\right) V Z\right)^{-1} Z^{T} V^{T}\left(I-P_{V S}\right) V Y
$$

with covariance matrix equal to

$$
\operatorname{Cov}(\hat{\tau})=\left(Z^{T} V^{T}\left(I-P_{V S}\right) V Z\right)^{-1},
$$

where $P_{V S}=V S\left[(V S)^{T} V S\right]^{-}(V S)^{T}$ is the projection matrix onto the vector space spanned by the columns of the matrix $V S,\left[(V S)^{T} V S\right]^{-}$is the generalized inverse of $(V S)^{T} V S$ and $V=\Sigma^{-1 / 2}$ is the whitening matrix so that $V \epsilon$ is white noise (uncorrelated errors). Denoting $\left(I-P_{V S}\right) V Z$ by $Z_{\perp}$, Eqs. (4.8) and (4.9) can be rewritten as

$$
\begin{aligned}
\hat{\tau} & =\left(Z_{\perp}^{T} Z_{\perp}\right)^{-1} Z_{\perp}^{T} V Y \\
\operatorname{Cov}(\hat{\tau}) & =\left(Z_{\perp}^{T} Z_{\perp}\right)^{-1} .
\end{aligned}
$$

Based on the covariance matrix, the $D$-optimality criterion can be used to evaluate the efficiency of a given design (Atkinson et al., 2007). The D-optimality criterion is based on the determinant or generalized variance of the covariance matrix of the estimators. An alternative to the $D$-optimality criterion is the $A$-optimality criterion which uses the trace of the covariance matrix of the estimators (Atkinson et al., 2007; Liu et al., 2001; Wager \& Nichols, 2003). A general advantage of the $D$-optimality criterion is that the optimal design will remain the same after linear transformation of the predictors in the design matrix. It is possible to rewrite Eq. (4.4) with $\tau^{*}=\left(\beta, \beta \theta_{1}, \ldots, \beta \theta_{5}\right)^{T}$. However, the determinant of the covariance matrix of $\hat{\tau}^{*}$ is equal to the determinant of the covariance matrix of $\hat{\tau}$, 
while this equality is not guaranteed for the $A$-optimality criterion. Therefore, we focused on the $D$-optimality criterion. The following criterion based on $D$-optimality was applied

$$
F=\operatorname{det}(\operatorname{Cov}(\hat{\tau}))^{-1 / r},
$$

where $r$ is the rank of $\operatorname{Cov}(\hat{\tau})$. The higher $F$ is, the better the design is.

Because the matrix $H\left(\theta_{0}\right)$ depends on the expansion point $\theta_{0}$, the obtained optimal design for a given value of $\theta_{0}$ is locally optimal. To handle the problem of local optimality the maximin criterion was applied for all designs $\xi_{1}$ in the design space $\Xi$ and all parameters $\theta_{0}$ in the parameter space $\Theta$. Here, the parameter space was the set of all considered values for the expansion point $\theta_{0}$ and is further described in Section 4.3.1. The maximin criterion was applied for each design type ( $m$-sequences, blocked designs etc.) separately. Thus, each design type had its own design space which was the set consisting of all considered designs for this design type. The parameter space however remained the same for all design types. The design space for the genetic algorithm consisted of all locally optimal designs obtained by the genetic algorithm as described in Section 4.3.2. Furthermore, Section 4.3.2 presents the design spaces for the other design types studied in this paper, such as $m$-sequences, blocked designs and rapid event-related designs.

All locally optimal designs of a design type, e.g., $m$-sequences, were compared to each other by the relative efficiency to finally select the maximin design from the locally optimal designs. We denote the locally optimal design obtained for a given expansion point $\theta_{0}$ by $\xi_{\theta_{0}}^{*}$. The relative efficiency of a locally optimal design $\xi_{1}$ versus the locally optimal design $\xi_{\theta_{0}}^{*}$ is given by

$$
\operatorname{RE}\left(\xi_{1} \mid \xi_{\theta_{0}}^{*}\right)=\frac{\operatorname{det}\left(\operatorname{Cov}\left(\hat{\tau}_{1}\right)\right)^{-1 / r}}{\operatorname{det}\left(\operatorname{Cov}\left(\hat{\tau}^{*}\right)\right)^{-1 / r}}
$$

with $\hat{\tau}_{1}$ and $\hat{\tau}^{*}$ being the GLS estimators obtained by designs $\xi_{1}$ and $\xi_{\theta_{0}}^{*}$ at the expansion point $\theta_{0}$. The covariance matrices of the GLS estimators $\operatorname{Cov}\left(\hat{\tau}_{1}\right)$ and $\operatorname{Cov}\left(\hat{\tau}^{*}\right)$ given by Eq. (4.9) depend on their respective designs since the matrix $X$ in $Z=X H\left(\theta_{0}\right)$ differs per design. The relative efficiency of the design $\xi$ versus the design $\xi_{\theta_{0}}^{*}$ in Eq. (4.13) depends on the expansion point $\theta_{0}$ because firstly the design $\xi_{1}$ is compared to the locally optimal design $\xi_{\theta_{0}}^{*}$ for the expansion point $\theta_{0}$ and secondly the GLS estimators obtained by the designs $\xi_{1}$ and $\xi_{\theta_{0}}^{*}$ are both evaluated at the expansion point $\theta_{0}$. The relative efficiency in Eq. (4.13) is a measure between 0 and 1 with a relative efficiency $\operatorname{RE}\left(\xi_{1} \mid \xi_{\theta_{0}}^{*}\right)$ of almost 0 for the design $\xi_{1}$ versus the design $\xi_{\theta_{0}}^{*}$ meaning that the design $\xi_{1}$ is very inefficient compared to the locally optimal design $\xi_{\theta_{0}}^{*}$ at the expansion point $\theta_{0}$. A relative efficiency close to 1 indicates that the design $\xi_{1}$ is almost as efficient as the locally optimal design $\xi_{\theta_{0}}^{*}$.

A locally optimal design is maximin $D$-optimal if it maximizes the smallest relative efficiency (Atkinson et al., 2007). Given a design $\xi_{1}$, the smallest relative efficiency $\min _{\theta_{0} \in \Theta} \operatorname{RE}\left(\xi_{1} \mid \xi_{\theta_{0}}^{*}\right)$ of this design $\xi_{1}$ over all expansion points $\theta_{0}$ in the parameter space $\Theta$, i.e., against all locally optimal designs $\xi_{\theta_{0}}^{*}$, is its worst relative efficiency. The maximin design MMD thus achieves the best-worst case for the relative efficiencies as it has the maximum minimum relative efficiency over all locally optimal designs $\xi_{1}$ in the design space $\Xi$ :

$$
\operatorname{MMD}=\arg \max _{\xi_{1} \in \Xi} \min _{\theta_{0} \in \Theta} \operatorname{RE}\left(\xi_{1} \mid \xi_{\theta_{0}}^{*}\right),
$$


When the maximin value MMV $=\max _{\xi_{1} \in \Xi} \min _{\theta_{0} \in \Theta} \operatorname{RE}\left(\xi_{1} \mid \xi_{\theta_{0}}^{*}\right)$ is high, the maximin design is very efficient versus the locally optimal designs at all parameter values in the parameter space.

The relative efficiency was further employed to compare the different maximin designs. The relative efficiency of a design $\xi_{1}$ versus a design $\xi_{2}$ is then a positive measure and is defined by

$$
\operatorname{RE}\left(\xi_{1} \mid \xi_{2}\right)=\frac{\operatorname{det}\left(\operatorname{Cov}\left(\hat{\tau}_{1}\right)\right)^{-1 / r}}{\operatorname{det}\left(\operatorname{Cov}\left(\hat{\tau}_{2}\right)\right)^{-1 / r}}
$$

The estimator $\hat{\tau}_{i}(i=1,2)$ is the GLS estimator for the design $\xi_{i}$ and depends on the design matrix belonging to design $\xi_{i}$. Using the model in Eq. (4.6), the relative efficiency in Eq. (4.15) depends on the expansion point $\theta_{0}$. The inverse of the relative efficiency gives the number of times that design $\xi_{1}$ has to be repeated to be as efficient as the design $\xi_{2}$.

\subsection{Methods}

The genetic algorithm was used to find locally optimal designs for the simultaneous nonlinear estimation of the parameters $a_{1}, b_{1}, c, a_{2}$ and $b_{2}$ for the double gamma function. From the locally optimal designs obtained by the GA (with one locally optimal design per considered expansion point of the Taylor approximation) the maximin design $\mathrm{MMD}_{\mathrm{GA}}$ was chosen. Furthermore, we obtained maximin designs from different specific design types which are commonly applied or known to be efficient, i.e., $m$-sequences, event-related designs with fixed ISI, blocked designs and rapid event-related designs with a geometric or uniform distribution for the ISI.

\subsubsection{Parameter space for expansion point}

The expansion point for the Taylor approximation was varied in $a_{1}, b_{1}, c, a_{2}$ and $b_{2}$. Note that while the estimation of the amplitude parameter $\beta$ is also optimized by maximizing Eq. (4.12), the locally optimal designs are not dependent on the value for $\beta$, but only on the expansion point $\theta_{0}$. Thus, only the HRF parameters $a_{1}, b_{1}, c, a_{2}$ and $b_{2}$ are varied for determination of the locally optimal designs. Parameter $a_{1}$ had values $4.8,5,5.2, b_{1}$ was varied from 0.9 to 1.3 in steps of $0.1, c$ was varied between 4 and 8 in steps of 1 and $a_{2}$ had values 14.5, 15 and 15.5. The parameter $b_{2}$ was equal to $0.9,1$ or 1.1 for $a_{2}=14.5$, equal to $0.9,1,1.1$ or 1.3 for $a_{2}=15$ and equal to $0.9,1,1.1$ or 1.3 for $a_{2}=15.5$. For each expansion point, the GA by Kao et al. (2009) was applied to find the locally optimal design which maximizes Eq. (4.12). The MATLAB code of Kao et al. (2009) was adapted to use the linearized model in Eq. (4.4). This resulted in 825 locally optimal designs from which the maximin design $\left(\mathrm{MMD}_{\mathrm{GA}}\right)$ was ascertained. The applied MATLAB code is available on request from the first author.

The chosen range for parameter values was obtained from the literature showing that the time to peak ( $\left.\mathrm{TTP}=a_{1} / b_{1}\right)$ of the hemodynamic response function can lie between 3 s and 7 s (Aguirre et al., 1998; Le et al., 2001; de Zwaart et al., 2005; Handwerker et al., 
2004) while the canonical values for the double gamma function are $a_{1}=5$ and $b_{1}=1$. The full width at half maximum (FWHM) of the first gamma function, given by $2.35 \cdot \sqrt{a_{1}} / b_{1}$ was found to lie in the interval [3 s, 6 s] (Bandettini \& Cox, 2000; Glover, 1999; Le et al., 2001; de Zwaart et al., 2005). The time to undershoot (TTU $=a_{2} / b_{2}$ ) was found to vary between $9 \mathrm{~s}$ and $18 \mathrm{~s}$ for real data (Aguirre et al., 1998; Friston et al., 2002; Handwerker et al., 2004) and simulations (Woolrich et al., 2004; Penny et al., 2004). The FWHM of the undershoot was assumed to be in the interval $7 \mathrm{~s}$ to $11 \mathrm{~s}$ because the canonical HRF with $a_{1}=5, b_{1}=1, c=6, a_{2}=15$ and $b_{2}=1$ has a FWHM of $9.10 \mathrm{~s}$ for the second gamma function. To reduce computation time we restricted the parameters to the values as given above which satisfy the conditions for TTP, TTU and FWHM of both gamma functions. Furthermore, the parameter steps were based on the results of a regression analysis which was performed to study the influence of the parameters on the accuracy of the Taylor approximation. Concluding from the results of the regression analysis, we chose smaller steps for $a_{1}$ and $b_{1}$ than for $a_{2}$ and $b_{2}$. As a result, the modeled range of the time to peak is $[3.69,5.78]$, the modeled FWHM of the peak is $[3.96,5.95]$, the modeled range of the time to undershoot is $[11.92,17.22]$ and the modeled FWHM of the undershoot is $[7.12,10.28]$. In Figure 4.1 the range of the hemodynamic response functions given by the parameter space is presented. The values for $a_{1}$ and $b_{1}$ in Figure 4.1a and the values for $a_{2}$ and $b_{2}$ in Figure $4.1 \mathrm{~b}$ were chosen so that the minima and maxima of the TTP, TTU and the FWHMs for the first and second gamma function were obtained.

\subsubsection{Design types}

The GA was applied for $Q=1$ stimulus type, $N=128$ events (including null events) and an autocorrelation $\rho=0.3$ within a first-order autoregressive (AR1) error $\epsilon$ (Kao et al., 2009; Maus et al., 2010b) so that the matrix $\Sigma$ in Eq. (4.1) has the form of an AR1 covariance matrix. The number of events refers here to the total number of events for the design and is a sum of the number of null events and number of stimulus trials. The exact number of null events or stimulus trials differed for each considered design. Furthermore, the following factors were considered: ISI $=2.5 \mathrm{~s}$ (here: the minimum time between two consecutive stimulus events) and $\mathrm{TR}=2 \mathrm{~s}$. This results in an effective sampling interval of $\mathrm{dT}=0.5 \mathrm{~s}$ for the hemodynamic response function (Josephs et al., 1997; Miezin et al., 2000) and in the number of time points $T=128 \cdot(2.5 \mathrm{~s}) /(2 \mathrm{~s})=160$. For the nuisance matrix $S$, a second order Legendre polynomial was assumed. The hemodynamic response function and its derivatives were calculated over a length of $40 \mathrm{~s}$ so that $k=40 / \mathrm{dT}+1=81$. The algorithm parameters were $G$ (size of population) $=20, q$ (percentage of mutation) $=1 \%, I$ (number of immigrants) $=4$ and $M_{g}$ (number of generations)=10,000 (Kao et al., 2009). The non-algorithmic parameters $Q, N, \rho$ and TR given above were also applied for the other design types and the minimum ISI was always chosen to be $2.5 \mathrm{~s}$.

The maximin design $\mathrm{MMD}_{m}$ was chosen from all possible $18 m$-sequences for 128 events (including null events) and for one stimulus type. Strictly speaking, the $m$-sequence lengths were 127 events but one null event was added at the sequence end to achieve stimulus frequency equal to 0.5. Note that the stimulus frequencies in this paper are determined on the "event design sequences" with $N=128$ events and not on the "scan design sequences" 


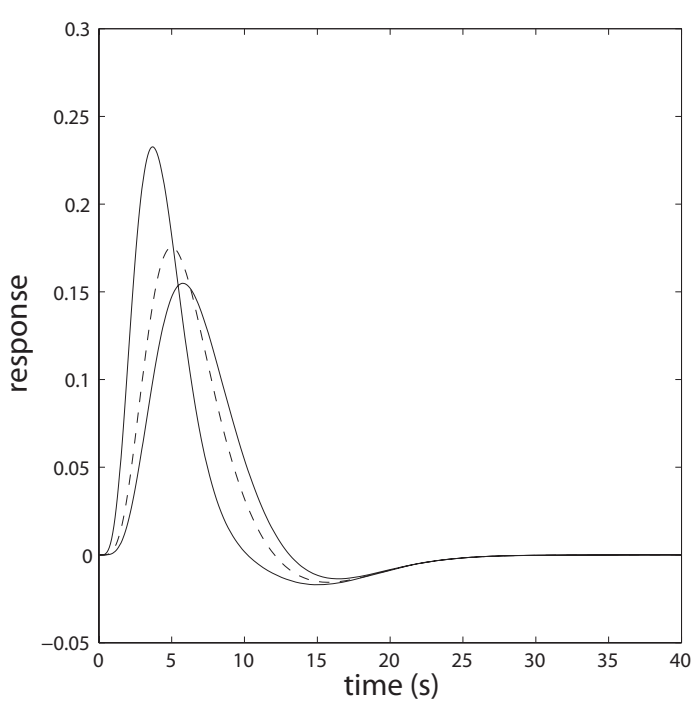

(a) Varying $a_{1}$ and $b_{1}$

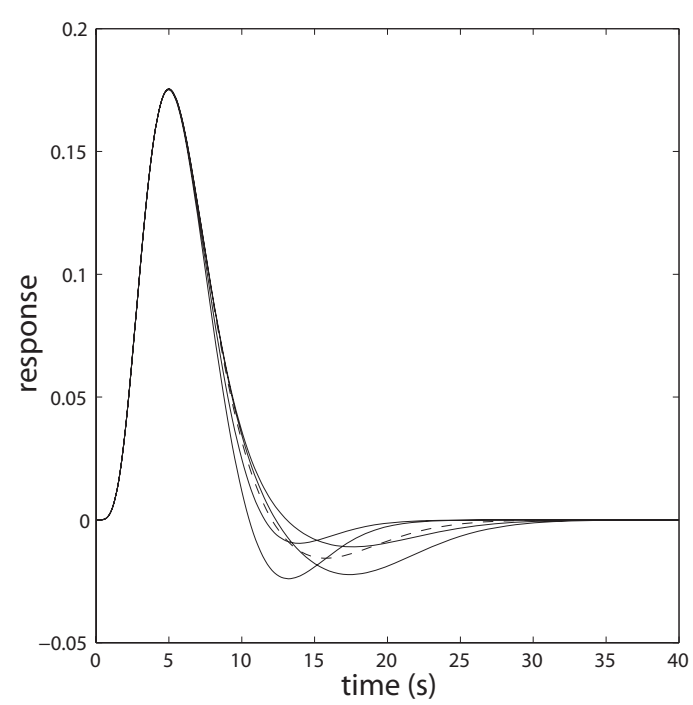

(b) Varying $c, a_{2}$ and $b_{2}$

Figure 4.1: Range of hemodynamic response functions captured by expansion points in the parameter space. In Figure 4.1a the hemodynamic response functions for $a_{1}=4.8$, $b_{1}=1.3$ and $a_{1}=5.2, b_{1}=0.9$ are shown by the solid lines $\left(c, a_{2}\right.$ and $b_{2}$ are fixed to $c=6$, $a_{2}=15$ and $\left.b_{2}=1\right)$. In Figure $4.1 \mathrm{~b}$ the hemodynamic response functions for $a_{2}=15.5$, $b_{2}=0.9, c=4$ or 8 and $a_{2}=15.5, b_{1}=1.3, c=4$ or 8 are shown by the solid lines $\left(a_{1}\right.$ and $b_{1}$ are fixed to $a_{1}=1$ and $\left.b_{1}=1\right)$. The dashed lines in Figures $4.1 \mathrm{a}$ and $4.1 \mathrm{~b}$ present the canonical hemodynamic response function $\left(a_{1}=5, b_{1}=1, c=6, a=15, b_{2}=1\right)$.

with $T=160$ time points as due to the minimum ISI of $2.5 \mathrm{~s}$ and the TR of $2 \mathrm{~s}$ not all stimulus events occurred at a scan. Furthermore, the maximin design $\mathrm{MMD}_{\mathrm{CI}}$ from several constant ISI designs and the maximin design $\mathrm{MMD}_{\mathrm{B}}$ from several blocked designs were chosen. Constant ISIs from $2.5 \mathrm{~s}, 5 \mathrm{~s}, \ldots, 17.5 \mathrm{~s}, 20 \mathrm{~s}$ and blocked designs with block length of $10 \mathrm{~s}, 12.5 \mathrm{~s}, \ldots, 27.5 \mathrm{~s}, 30 \mathrm{~s}$ and $60 \mathrm{~s}$ were considered. The blocked designs started with a stimulus block followed by a rest block of same length. Some block lengths resulted in remaining time points at the end of the time series. These remaining time points were either filled completely with rest events or completely with stimulus events. In total, 18 blocked designs were considered. All constant ISI designs and blocked designs were assumed to have a total number of events (including null events) of 128 .

Additionally, maximin designs from random event-related designs were chosen. Commonly applied and proven to be efficient are a uniform or geometric distribution for the ISI (Hagberg et al., 2001; Ollinger et al., 2001; Liu et al., 2001; Bianciardi et al., 2004). The maximin design $\mathrm{MMD}_{\text {geo }}$ was chosen out of realizations of designs with a geometric distribution for ISI. Such a distribution can be described by three parameters: ISI $_{\text {min }}$ which gives the smallest possible time between two events, $p$ which models the probability of the number of time points between two events and ISI $_{\text {step }}$ which is the step of ISIs. The random variable $X$ which represents the number of ISI steps until the next stimulus event has probability $P(X=x)=(1-p)^{x} p$. The random variable ISI is given by $\mathrm{ISI}_{\min }+X \cdot \mathrm{ISI}_{\text {step }}$. 
The 27 applied parameter sets for the geometric distribution are presented in Table 4.1.

Table 4.1: Considered geometric designs. Design G5 is the maximin design.

\begin{tabular}{ccccccccccc}
\hline & & \multicolumn{10}{c}{$p$} \\
\cline { 3 - 10 } ISI $_{\min }$ & ISI $_{\text {step }}$ & 0.1 & 0.2 & 0.3 & 0.4 & 0.5 & 0.6 & 0.7 & 0.8 & 0.9 \\
\hline $2.5 \mathrm{~s}$ & $2.5 \mathrm{~s}$ & G1 & G2 & G3 & G4 & G5 & G6 & G7 & G8 & G9 \\
$5.0 \mathrm{~s}$ & $2.5 \mathrm{~s}$ & G10 & G11 & G12 & G13 & G14 & G15 & G16 & G17 & G18 \\
$5.0 \mathrm{~s}$ & $5.0 \mathrm{~s}$ & G19 & G20 & G21 & G22 & G23 & G24 & G25 & G26 & G27 \\
\hline
\end{tabular}

Each parameter set refers to a single design and we computed 200 realizations of each design so that 5400 sequences (27 designs) were obtained. The maximin design and the maximin design sequence were chosen in a two-step procedure.

In the first step, the maximin design and its parameter set were determined. The first step included determination of the locally optimal sequences from the 5400 sequences (one locally optimal design per expansion point), calculation of the relative efficiencies of all sequences against the locally optimal sequences and calculation of the minimum relative efficiency of each sequence. Finally, for each design the average minimum relative efficiency over all 200 realizations was calculated. The design with highest mean minimum relative efficiency is the maximin design. Averaging was performed to find a maximin design which generally results in sequences with high minimum relative efficiencies instead of finding a maximin design with by coincidence one highly efficient sequence.

In the second step, one design sequence was chosen from the realizations of the maximin design to be the maximin design sequence. The second step consisted of determination of the locally optimal sequences among the 200 sequences, calculation of the relative efficiencies and minimum relative efficiencies. The stimulus sequence with highest minimum relative efficiency among the 200 sequences was chosen to be the maximin design sequence.

Following the same two-step procedure as for the geometric distribution, the maximin design $\mathrm{MMD}_{\text {uni }}$ was chosen out of 13 designs with a uniform distribution for ISI. 400 realizations of each design were performed so that 5200 sequences in total were obtained. According to the two-step procedure, the maximin design sequence was chosen out of the 400 realizations of the maximin design. The applied ISIs for the uniform designs are indicated in Table 4.2.

Computation times for the different maximin designs under linearity were measured on a computer with Intel Core i7 processor, 2.67 GHz and 6.00 GB RAM. To model nonlinearity, we assumed a reduction in amplitude of $30 \%$ for an ISI of $2.5 \mathrm{~s}$ (Huettel \& McCarthy, 2000) and of $21 \%$ for an ISI of $5 \mathrm{~s}$ (Miezin et al., 2000) within the design matrix $X$. Using the same design types, parameter sets and the same two-step procedure as described for linearity, maximin designs under the assumption of nonlinearity were obtained. We will refer to these designs as $\mathrm{MMD}_{\mathrm{GANL}}, \mathrm{MMD}_{m \mathrm{NL}}, \mathrm{MMD}_{\mathrm{CINL}}, \mathrm{MMD}_{\mathrm{BNL}}, \mathrm{MMD}_{\text {geoNL }}$ and $\mathrm{MMD}_{\text {uniNL }}$. 
Table 4.2: Applied ISIs for uniform designs ( $\mathrm{x}$ indicates the applied ISIs). Design U6 is the maximin design.

\begin{tabular}{lccccccccccccc}
\hline & \multicolumn{10}{c}{ Designs } \\
\cline { 2 - 14 } & $\mathrm{U} 1$ & $\mathrm{U} 2$ & $\mathrm{U} 3$ & $\mathrm{U} 4$ & $\mathrm{U} 5$ & $\mathrm{U} 6$ & $\mathrm{U} 7$ & $\mathrm{U} 8$ & $\mathrm{U} 9$ & $\mathrm{U} 10$ & $\mathrm{U} 11$ & $\mathrm{U} 12$ & $\mathrm{U} 13$ \\
\hline $2.5 \mathrm{~s}$ & $\mathrm{x}$ & $\mathrm{x}$ & $\mathrm{x}$ & $\mathrm{x}$ & $\mathrm{x}$ & $\mathrm{x}$ & $\mathrm{x}$ & & & & & & \\
$5.0 \mathrm{~s}$ & $\mathrm{x}$ & $\mathrm{x}$ & $\mathrm{x}$ & $\mathrm{x}$ & $\mathrm{x}$ & & & $\mathrm{x}$ & $\mathrm{x}$ & $\mathrm{x}$ & $\mathrm{x}$ & $\mathrm{x}$ & $\mathrm{x}$ \\
$7.5 \mathrm{~s}$ & $\mathrm{x}$ & $\mathrm{x}$ & $\mathrm{x}$ & $\mathrm{x}$ & $\mathrm{x}$ & $\mathrm{x}$ & $\mathrm{x}$ & & & $\mathrm{x}$ & $\mathrm{x}$ & $\mathrm{x}$ & $\mathrm{x}$ \\
$10.0 \mathrm{~s}$ & & $\mathrm{x}$ & $\mathrm{x}$ & $\mathrm{x}$ & $\mathrm{x}$ & & & $\mathrm{x}$ & $\mathrm{x}$ & $\mathrm{x}$ & $\mathrm{x}$ & $\mathrm{x}$ & $\mathrm{x}$ \\
$12.5 \mathrm{~s}$ & & & $\mathrm{x}$ & $\mathrm{x}$ & $\mathrm{x}$ & $\mathrm{x}$ & $\mathrm{x}$ & & & & $\mathrm{x}$ & $\mathrm{x}$ & $\mathrm{x}$ \\
$15.0 \mathrm{~s}$ & & & & $\mathrm{x}$ & $\mathrm{x}$ & & & $\mathrm{x}$ & $\mathrm{x}$ & & & $\mathrm{x}$ & $\mathrm{x}$ \\
$17.5 \mathrm{~s}$ & & & & $\mathrm{x}$ & & $\mathrm{x}$ & & & & & & $\mathrm{x}$ \\
$20.0 \mathrm{~s}$ & & & & & & & & $\mathrm{x}$ & & & & \\
\hline
\end{tabular}

\subsection{Results}

The maximin design $\mathrm{MMD}_{\mathrm{GA}}$ obtained from the genetic algorithm, proved to be most efficient in comparison to the other maximin designs $\left(\mathrm{MMD}_{m}, \mathrm{MMD}_{\mathrm{CI}}, \mathrm{MMD}_{\mathrm{B}}, \mathrm{MMD}_{\text {geo }}\right.$ and $\mathrm{MMD}_{\text {uni }}$ ) obtained under the assumption of linearity of the HRF. Likewise, the maximin design $\mathrm{MMD}_{\mathrm{GANL}}$ was most efficient under the assumption of nonlinearity of the HRF. Therefore, we restrict our design comparison in Sections 4.4.1 and 4.4.2 to the pairwise comparison of the maximin designs obtained by the GA to the maximin designs obtained by $m$-sequences, constant ISI designs, blocked designs and designs with geometric or uniform distribution for ISI under the assumption of linearity or nonlinearity.

The minimum relative efficiency of the maximin designs within their own design type and the range of relative efficiencies against the $\mathrm{MMD}_{\mathrm{GA}}$ or $\mathrm{MMD}_{\mathrm{GANL}}$ over the expansion points are given in Tables 4.3 and 4.4. Furthermore, the relative efficiencies of each maximin design from the specific design types against the maximin design $\mathrm{MMD}_{\mathrm{GA}}$ are illustrated in Figure 4.2. Note that the calculation of the relative efficiencies against $\mathrm{MMD}_{\mathrm{GA}}$ and $\mathrm{MMD}_{\mathrm{GANL}}$ in Figure 4.2, Tables 4.3 and 4.4 is based on Eq. (4.15) and not on the by Eq. (4.13) calculated relative efficiencies, which were used to determine the maximin design from the locally optimal designs of each design type.

After the presentation of the results for the different maximin designs, the maximin design $\mathrm{MMD}_{\mathrm{GA}}$ is compared in Section 4.4.3 to the optimal design for HRF estimation, using a linear model with the stimulus convolution matrix as design matrix, and to the optimal design for detection of task-related activation, using a linear model where the regressors are obtained by multiplication of the stimulus convolution matrix with the sampled HRF. The latter design will be called the optimal design for detection power whereas the former design will be called the optimal design for estimation efficiency. The corresponding models will be named detection power model or estimation efficiency model. These comparisons are interesting for evaluation of the efficiency loss when the model and optimality criterion do not match with the ultimate data analysis of an fMRI design. Linearity of the HRF was assumed for the calculation of both designs which were obtained by applying the original code for the genetic algorithm of Kao et al. (2009). More details are given in Section 4.4.3. 


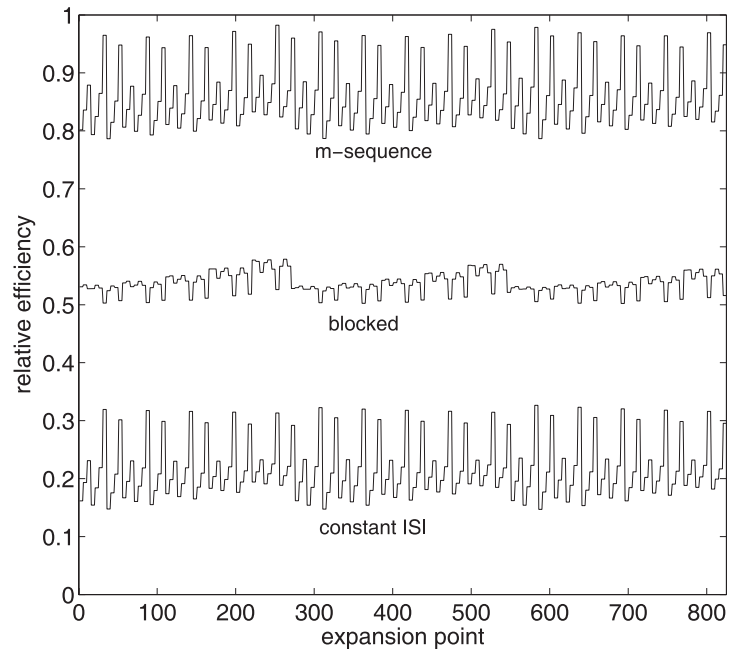

(a) $m$-sequences, blocked and constant ISI

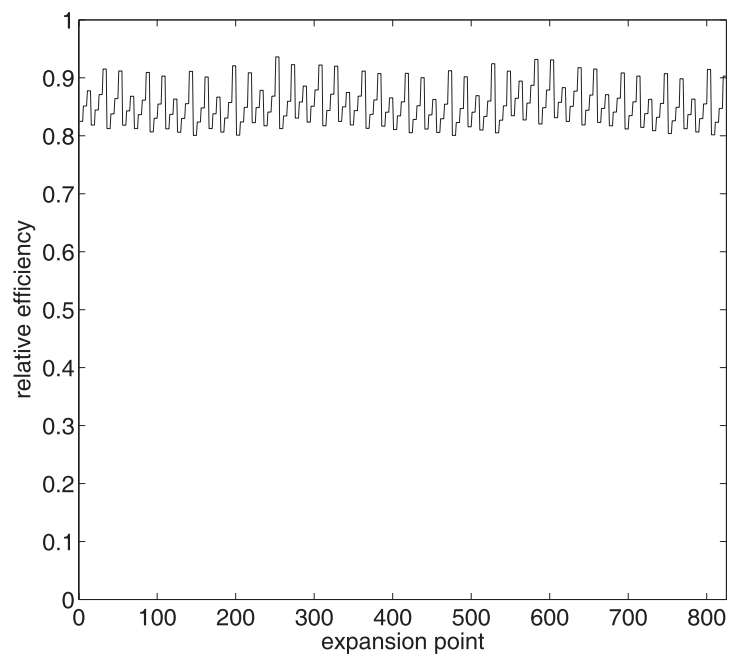

(c) Uniform

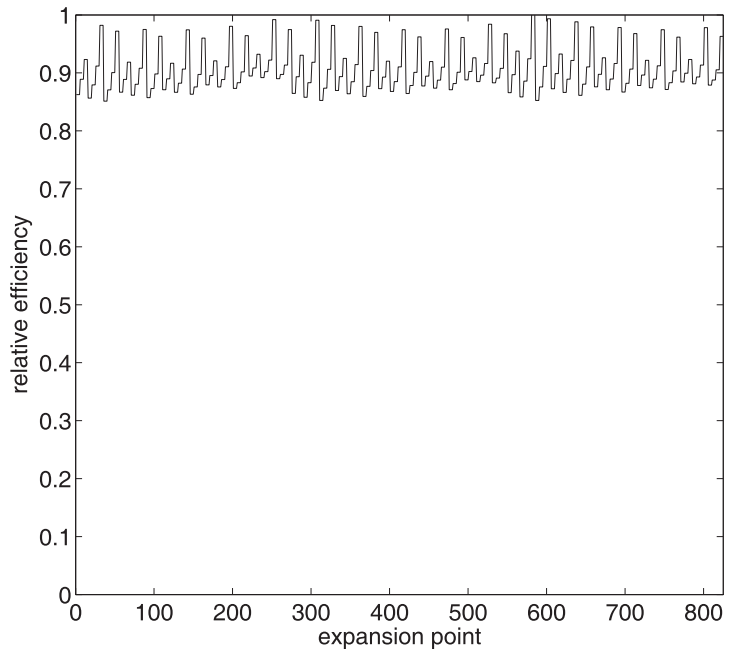

(b) Geometric

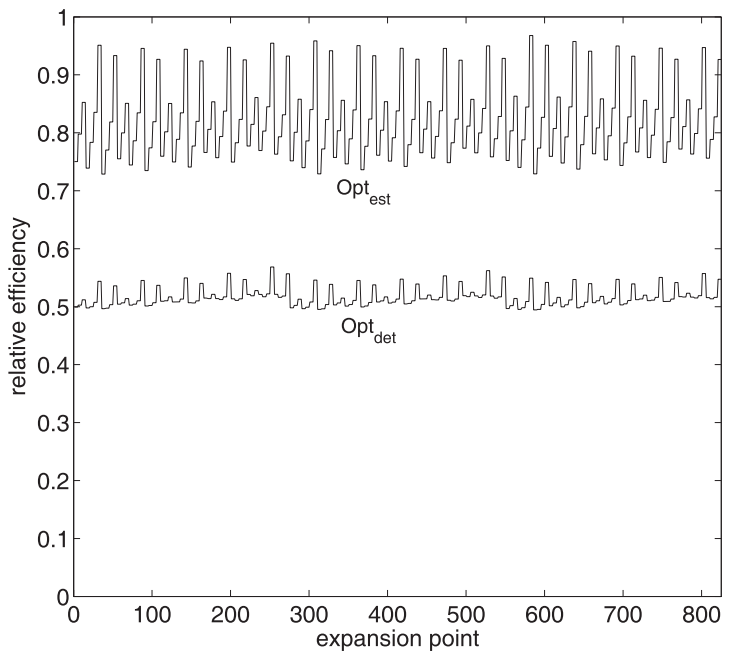

(d) Optimal designs

Figure 4.2: Relative efficiency of maximin design based on different design types and optimal designs versus $\mathrm{MMD}_{\mathrm{GA}}$

\subsubsection{Linearity of hemodynamic response function}

The maximin design $\mathrm{MMD}_{\mathrm{GA}}$ presented in Figure 4.3a is a mixture of a blocked and a rapid event-related design, as it contains task and null blocks while other parts of the design sequence have an event-related form. The maximin design $\mathrm{MMD}_{m}$ in Figure 4.3b is more similar to a rapid event-related design than the maximin design $\mathrm{MMD}_{\mathrm{GA}}$. The maximin design $\mathrm{MMD}_{\mathrm{CI}}$ for the constant ISI designs is the slow event-related design with 17.5 s ISI (see Figure 4.3c) and the maximin design $\mathrm{MMD}_{\mathrm{B}}$ for the blocked designs has block length $25 \mathrm{~s}$ and one stimulus block at the end (see Figure 4.3d). The maximin geometric ISI design $\mathrm{MMD}_{\text {geo }}$ in Figure 4.3e is the design with $\mathrm{ISI}_{\text {min }}=\mathrm{ISI}_{\text {step }}=2.5 \mathrm{~s}$, $p=0.5$ and the maximin design $\mathrm{MMD}_{\text {uni }}$ shown in Figure 4.3f is the design with ISIs 2.5 $\mathrm{s}, 7.5 \mathrm{~s}$ and $12.5 \mathrm{~s}$. 


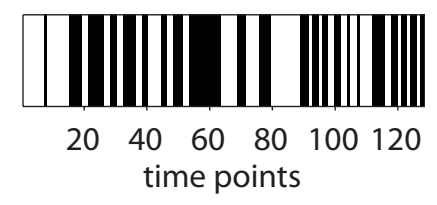

(a) Genetic algorithm

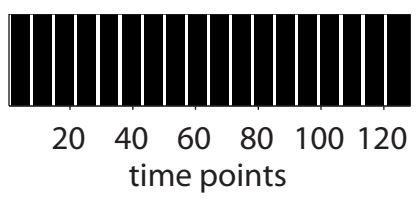

(c) Constant ISI

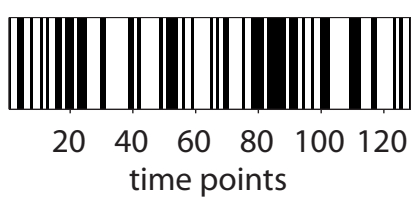

(e) Geometric

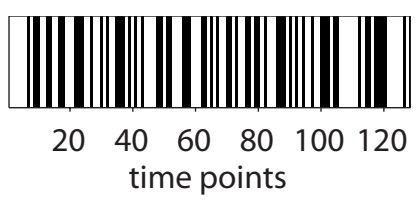

(g) Estimation efficiency

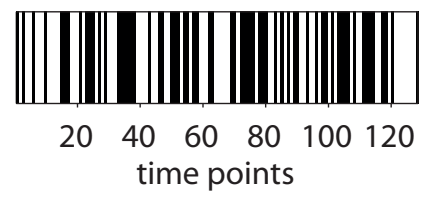

(b) $m$-sequence

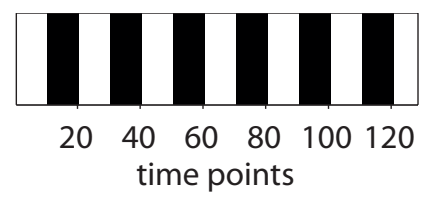

(d) Blocked

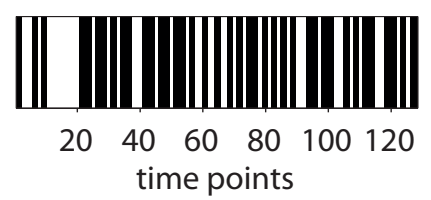

(f) Uniform

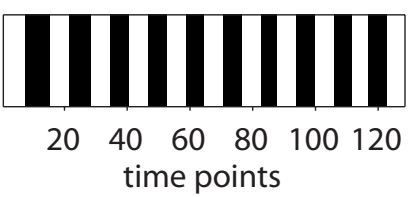

(h) Detection power

Figure 4.3: Maximin designs and optimal designs, white indicates a stimulus, black indicates no stimulus. The time between two time points is here $2.5 \mathrm{~s}$ which is the employed minimum ISI. TR was equal to $2 \mathrm{~s}$.

Table 4.3: Properties of maximin designs under linearity, $\mathrm{BL}=$ block length. Computation times were determined on a computer with Intel Core i7 processor, 2.67 GHz and 6.00 GB RAM. Letter A stands for the stimulus and 0 represents null event.

\begin{tabular}{|c|c|c|c|c|c|c|c|}
\hline & & $\begin{array}{c}\text { Mean } \\
\text { BL }\end{array}$ & $\begin{array}{c}\text { Mean } \\
\text { ISI }\end{array}$ & Frequency & Min RE & $\begin{array}{l}\text { RE versus } \\
\text { MMD }_{\mathrm{GA}}\end{array}$ & $\begin{array}{c}\text { Computation } \\
\text { time }\end{array}$ \\
\hline \multirow{2}{*}{$\begin{array}{l}\text { Genetic } \\
\text { algorithm }\end{array}$} & 0 & $7.16 \mathrm{~s}$ & \multirow{2}{*}{$4.88 \mathrm{~s}$} & 0.49 & \multirow{2}{*}{0.93} & \multirow{2}{*}{1.00} & \multirow{2}{*}{$45.83 \mathrm{~h}$} \\
\hline & $\mathrm{A}$ & $7.39 \mathrm{~s}$ & & 0.51 & & & \\
\hline \multirow{2}{*}{$m$-sequence } & 0 & $4.85 \mathrm{~s}$ & \multirow{2}{*}{$4.96 \mathrm{~s}$} & 0.50 & \multirow{2}{*}{0.99} & \multirow{2}{*}{$0.79-0.98$} & \multirow{2}{*}{$18 \mathrm{~s}$} \\
\hline & A & $5.00 \mathrm{~s}$ & & 0.50 & & & \\
\hline \multirow{2}{*}{ Blocked } & 0 & $25.00 \mathrm{~s}$ & \multirow{2}{*}{$4.74 \mathrm{~s}$} & 0.47 & \multirow{2}{*}{0.89} & \multirow{2}{*}{$0.50-0.58$} & \multirow{2}{*}{$18 \mathrm{~s}$} \\
\hline & A & $24.29 \mathrm{~s}$ & & 0.53 & & & \\
\hline \multirow{2}{*}{ Constant ISI } & 0 & $15.28 \mathrm{~s}$ & \multirow{2}{*}{$17.50 \mathrm{~s}$} & 0.86 & \multirow{2}{*}{0.95} & \multirow{2}{*}{$0.15-0.33$} & \multirow{2}{*}{$8 \mathrm{~s}$} \\
\hline & $\mathrm{A}$ & $2.50 \mathrm{~s}$ & & 0.14 & & & \\
\hline \multirow{2}{*}{ Geometric } & 0 & $4.83 \mathrm{~s}$ & \multirow{2}{*}{$4.53 \mathrm{~s}$} & 0.45 & 0.97 (step 1) & \multirow{2}{*}{$0.85-1.00$} & $642 \mathrm{~s}$ \\
\hline & A & $6.03 \mathrm{~s}$ & & 0.55 & 0.99 (step 2) & & (all steps) \\
\hline \multirow{2}{*}{ Uniform } & 0 & $7.05 \mathrm{~s}$ & \multirow{2}{*}{$6.35 \mathrm{~s}$} & 0.62 & 0.95 (step 1) & \multirow{2}{*}{$0.80-0.94$} & \multirow{2}{*}{$\begin{array}{c}629 \mathrm{~s} \\
\text { (all steps) }\end{array}$} \\
\hline & $\mathrm{A}$ & $4.54 \mathrm{~s}$ & & 0.38 & 0.9976 (step 2) & & \\
\hline
\end{tabular}


The properties of the maximin designs are presented in Table 4.3. Furthermore, the minimum relative efficiency of the maximin designs within their own design class and the range of relative efficiencies against the $\mathrm{MMD}_{\mathrm{GA}}$ over the expansion points are given. The range of relative efficiencies against the maximin design $\mathrm{MMD}_{\mathrm{GA}}$ is also illustrated in Figure 4.2. Further details will now be discussed per design type.

\subsubsection{Genetic algorithm}

The maximin design $\mathrm{MMD}_{\mathrm{GA}}$ has a high frequency of ISI $2.5 \mathrm{~s}$ and some longer ISIs of $5 \mathrm{~s}, 7.5 \mathrm{~s}, 10 \mathrm{~s}, 12.5 \mathrm{~s}, 15 \mathrm{~s}$ and $27.5 \mathrm{~s}$ as can be seen in Figure 4.4. The longer ISIs indicate null blocks. Null events had a slightly lower frequency than stimulus events in the maximin design $\mathrm{MMD}_{\mathrm{GA}}$ (see Table 4.3). This was also the case for all the locally optimal designs, except two which had equal frequencies. The minimum relative efficiencies of the 825 locally optimal designs were on average 0.87 , and the range was from 0.80 to 0.93 . The maximin design $\mathrm{MMD}_{\mathrm{GA}}$ was obtained for $a_{1}=4.8, b_{1}=1.1, c=4, a_{2}=15.5$ and $b_{2}=1.1$, and its minimum relative efficiency was equal to 0.93 . The minimum relative efficiency of the locally optimal design for the canonical expansion point $\left(a_{1}=5, b_{1}=1\right.$, $c=6, a_{2}=15$ and $b_{2}=1$ ) was equal to 0.88 .

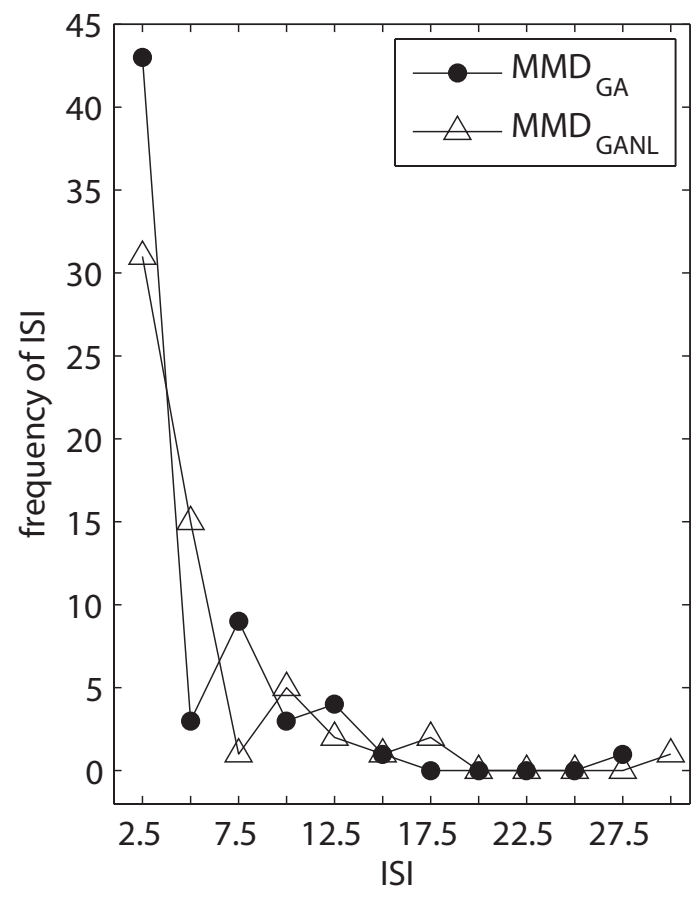

Figure 4.4: Frequency of ISI for maximin designs $\mathrm{MMD}_{\mathrm{GA}}$ and $\mathrm{MMD}_{\mathrm{GANL}}$

In Figure 4.5 the convergence of the genetic algorithm for all expansion points is shown. It can be seen that for most expansion points the genetic algorithm converged very quickly to $99 \%$ of its maximum and final value $F_{\max }$ obtained for 10,000 generations. For some expansion points however reaching $99 \%$ of $F_{\max }$ needed more generations. The worst convergence was for expansion point number 668 with 3507 generations to reach $99 \%$ of $F_{\max }$. 


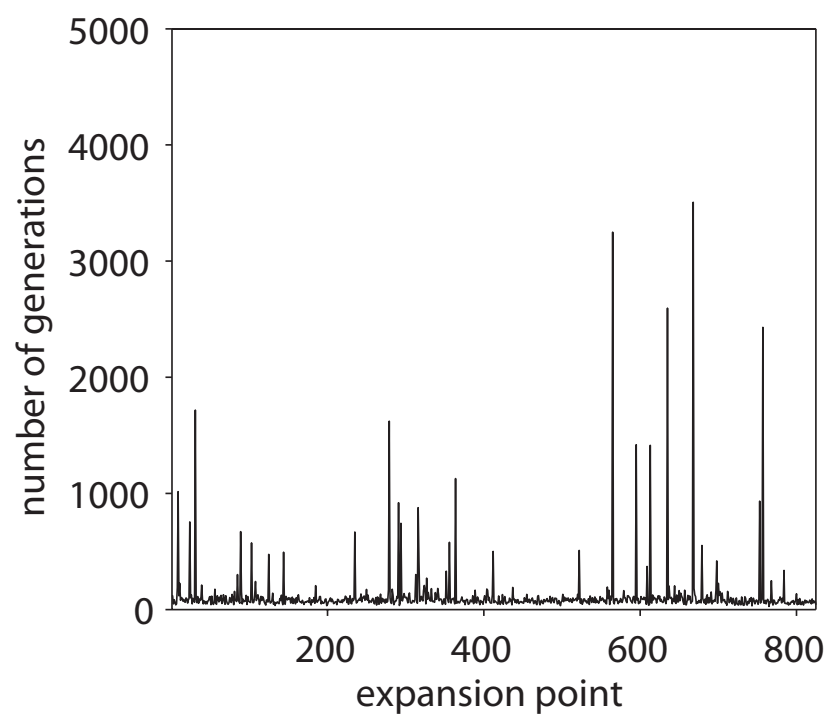

Figure 4.5: Convergence of the genetic algorithm for all expansion points. The number of generations is shown which is needed at a given expansion point to reach $99 \%$ of the final value $F_{\max }$ (maximum value of design criterion $F$ ) after 10,000 generations. On the $x$-axis the expansion points are given and on the $y$-axis the number of generations is shown.

\subsubsection{2 $m$-sequence, constant ISI designs, blocked designs}

The maximin design $\mathrm{MMD}_{\mathrm{GA}}$ was compared to the maximin design $\mathrm{MMD}_{m}$ of the $m$ sequences by calculating the relative efficiency as in Eq. (4.15) of the maximin design $\mathrm{MMD}_{m}$ versus $\mathrm{MMD}_{\mathrm{GA}}$ at each expansion point. Likewise, the relative efficiency of the maximin design $\mathrm{MMD}_{\mathrm{B}}$ and $\mathrm{MMD}_{\mathrm{CI}}$ versus $\mathrm{MMD}_{\mathrm{GA}}$ was determined. Figure 4.2a shows that the maximin design $\mathrm{MMD}_{m}$ was slightly less efficient than the maximin design $\mathrm{MMD}_{\mathrm{GA}}$ at all expansion points. It can be seen in Figure $4.2 \mathrm{a}$ that the maximin design $\mathrm{MMD}_{\mathrm{CI}}$ had a low relative efficiency and the maximin design $\mathrm{MMD}_{\mathrm{B}}$ a medium relative efficiency.

\subsubsection{Geometric distribution}

In Figure 4.6a the minimum relative efficiencies of the 5400 random sequences versus the locally optimal designs are presented. On the $x$-axis the 27 designs with geometric distribution for ISI are given (see Table 4.1) and on the $y$-axis the range of minimum relative efficiencies is indicated. For each design a boxplot of the 200 minimum relative efficiencies for this design is shown. The vertical lines between the boxplots indicate the three sets of nine designs which have the same $\mathrm{ISI}_{\text {min }}$ and $\mathrm{ISI}_{\text {step }}$ but differ in probability $p$. Figure 4.6a shows that for the geometric distribution, designs with middle probability around $p=0.5$ perform better with respect to minimum relative efficiency than designs with high and low $p$ as long as the other factors such as ISI $_{\text {min }}$ are kept constant. Furthermore, designs with high or low $p$ have more variability in the minimum relative efficiencies. A small ISI $_{\text {min }}$ is more efficient than a higher ISI $_{\text {min }}$. The maximin design for the geometric distribution which is shown in Figure 4.3e had an ISI $_{\min }$ of $2.5 \mathrm{~s}$ with possible ISIs in steps of $2.5 \mathrm{~s}$ and $p$ was equal to 0.5 (design G5 in Figure 4.6a). This results in a theoretical mean ISI of $5 \mathrm{~s}$. 


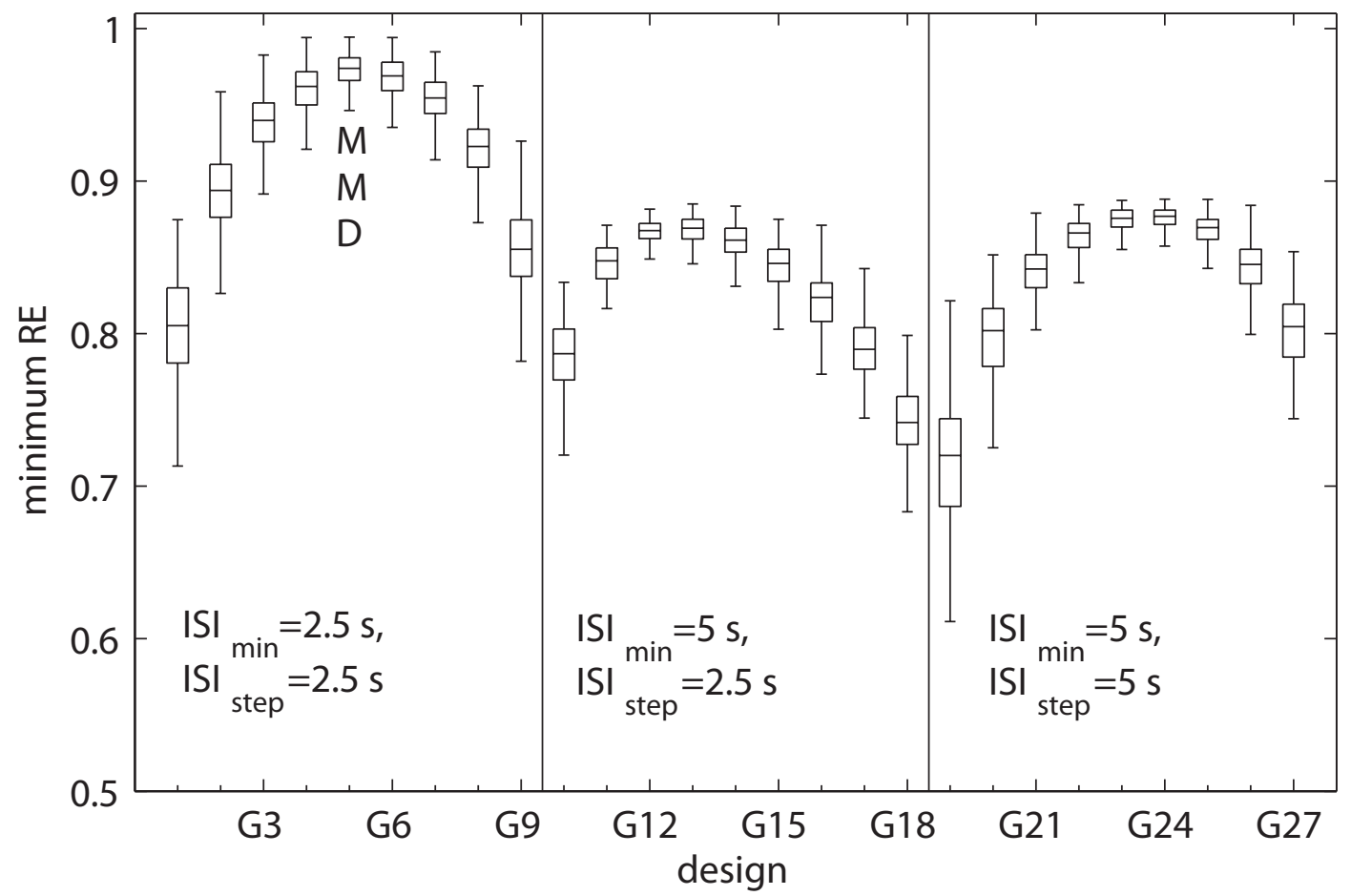

(a) Boxplots of minimum relative efficiency (RE) for designs with a geometric distribution for ISI. On the $x$-axis the 27 designs G1 till G27 are indicated (see Table 4.1 for further information).

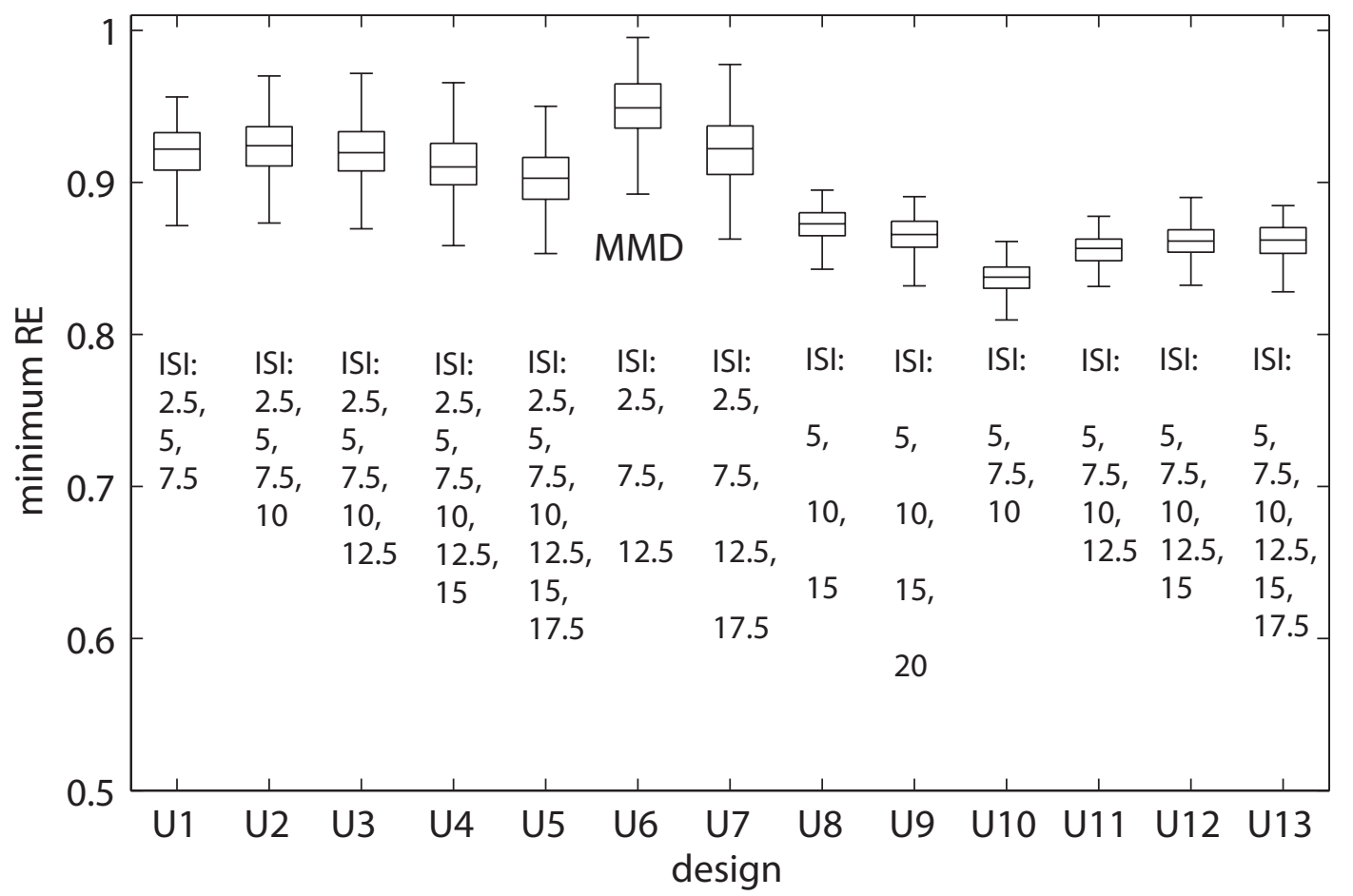

(b) Boxplots of minimum relative efficiency (RE) for designs with a uniform distribution for ISI. On the $x$-axis the different 13 designs $\mathrm{U} 1$ till U13 are given (see Table 4.2 for further information).

Figure 4.6: The boxes give the median and $25 \%$ and $75 \%$ percentile of the 200 (geometric) or 400 (uniform) minimum relative efficiencies per design versus the locally optimal designs. The whiskers show the most extreme data points (minimum relative efficiencies) which differ from the median by maximally 1.5 - interquartile range. MMD denotes the maximin design. 
Figure 4.2b illustrates that the maximin design $\mathrm{MMD}_{\text {geo }}$ performs well against the maximin design $\mathrm{MMD}_{\mathrm{GA}}$.

\subsubsection{Uniform distribution}

In Figure 4.6b the boxplots of minimum relative efficiencies for each of the 13 uniform designs are shown. The minimum relative efficiencies are calculated by comparison of the 5200 random sequences versus the locally optimal sequences. The maximin design in Figure 4.3f for the uniform distribution was the design with ISIs $2.5 \mathrm{~s}, 7.5 \mathrm{~s}$ and 12.5 $\mathrm{s}$ (design U6 in Figure 4.6b). Figure 4.6b illustrates that designs with minimum ISI 2.5 $\mathrm{s}$ have higher minimum relative efficiencies. The maximin design $\mathrm{MMD}_{\text {uni }}$ performs well versus $\mathrm{MMD}_{\mathrm{GA}}$ but not as well as $\mathrm{MMD}_{\text {geo }}$, see Figure 4.2c.

\subsubsection{Nonlinearity of hemodynamic response function}

The properties of the maximin designs, their minimum relative efficiencies within their own design class and their relative efficiencies versus the maximin design $\mathrm{MMD}_{\mathrm{GANL}}$ can be seen in Table 4.4. The main difference between the results for linearity and nonlinearity was that the maximin design for the genetic algorithm $\mathrm{MMD}_{\mathrm{GANL}}$ and the maximin design based on the geometric distribution $\mathrm{MMD}_{\text {geoNL }}\left(p=0.4, \mathrm{ISI}_{\text {min }}=2.5 \mathrm{~s}, \mathrm{ISI}_{\text {step }}=2.5 \mathrm{~s}\right)$ had a higher mean ISI than the corresponding maximin designs $\mathrm{MMD}_{\mathrm{GA}}$ and $\mathrm{MMD}_{\text {geo }}$ for linearity. This can be explained by the fact than an ISI of $2.5 \mathrm{~s}$ is less efficient under nonlinearity and thus less frequent in the design $\mathrm{MMD}_{\text {geoNL }}$ and the maximin design $\mathrm{MMD}_{\mathrm{GANL}}$. Among the 18 possible $m$-sequences $4.96 \mathrm{~s}$ was the highest mean ISI and both maximin designs $\mathrm{MMD}_{\mathrm{mNL}}$ and $\mathrm{MMD}_{m}$ had this mean ISI. For the maximin design $\mathrm{MMD}_{\text {uniNL}}$, the mean ISI was lower than for the maximin design $\mathrm{MMD}_{\text {uni }}$ which is in contrast to the designs mentioned above. This different relation in mean ISI might be explained by our specific samples. Generating another 400 realizations of the maximin uniform designs resulted in different mean ISIs, and the mean ISI was lower for the new maximin design $\mathrm{MMD}_{\text {uni }}$ than

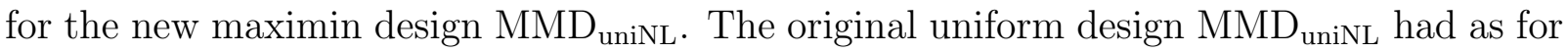
linearity the ISIs $2.5 \mathrm{~s}, 7.5 \mathrm{~s}$ and $12.5 \mathrm{~s}$. The distribution of ISI for the maximin design $\mathrm{MMD}_{\mathrm{GANL}}$ can be seen in Figure 4.4.

The other maximin designs under nonlinearity of the HRF were as follows. The maximin constant ISI design had an ISI of $17.5 \mathrm{~s}$ and was thus unaffected by the nonlinearity for ISIs $2.5 \mathrm{~s}$ and $5 \mathrm{~s}$. Another minor difference between linearity and nonlinearity was that the maximin blocked design $\mathrm{MMD}_{\mathrm{BNL}}$ had block length $22.5 \mathrm{~s}$ instead of $25 \mathrm{~s}$. Like for linearity, the blocked design $\mathrm{MMD}_{\mathrm{BNL}}$ had a stimulus block at the end. 
Table 4.4: Properties of maximin designs under nonlinearity, $\mathrm{BL}=$ block length. Letter A stands for the stimulus and 0 represents null event.

\begin{tabular}{|c|c|c|c|c|c|c|}
\hline & & Mean BL & Mean ISI & Frequency & Min RE & $\begin{array}{l}\text { RE versus } \\
\text { MMD }_{\text {GANL }}\end{array}$ \\
\hline \multirow{2}{*}{$\begin{array}{l}\text { Genetic } \\
\text { algorithm }\end{array}$} & 0 & $6.16 \mathrm{~s}$ & \multirow{2}{*}{$5.43 \mathrm{~s}$} & 0.54 & \multirow{2}{*}{0.95} & \multirow{2}{*}{1.00} \\
\hline & $\mathrm{A}$ & $5.27 \mathrm{~s}$ & & 0.46 & & \\
\hline \multirow{2}{*}{$m$-sequence } & 0 & $4.85 \mathrm{~s}$ & \multirow{2}{*}{$4.96 \mathrm{~s}$} & 0.50 & \multirow{2}{*}{0.99} & \multirow{2}{*}{$0.84-0.98$} \\
\hline & $\mathrm{A}$ & $5.00 \mathrm{~s}$ & & 0.50 & & \\
\hline \multirow{2}{*}{ Blocked } & 0 & $22.50 \mathrm{~s}$ & \multirow{2}{*}{$4.96 \mathrm{~s}$} & 0.49 & \multirow{2}{*}{0.91} & \multirow{2}{*}{$0.49-0.55$} \\
\hline & A & $20.31 \mathrm{~s}$ & & 0.51 & & \\
\hline \multirow{2}{*}{ Constant ISI } & 0 & $15.28 \mathrm{~s}$ & \multirow{2}{*}{$17.50 \mathrm{~s}$} & 0.86 & \multirow{2}{*}{0.95} & \multirow{2}{*}{$0.23-0.44$} \\
\hline & A & $2.50 \mathrm{~s}$ & & 0.14 & & \\
\hline \multirow{2}{*}{ Geometric } & 0 & $6.72 \mathrm{~s}$ & \multirow{2}{*}{$6.38 \mathrm{~s}$} & 0.61 & $0.97($ step 1) & \multirow{2}{*}{ 0.89-0.98 } \\
\hline & $\mathrm{A}$ & $4.31 \mathrm{~s}$ & & 0.39 & 0.9955 (step 2) & \\
\hline \multirow{2}{*}{ Uniform } & 0 & $7.12 \mathrm{~s}$ & \multirow{2}{*}{$5.71 \mathrm{~s}$} & 0.58 & 0.96 (step 1) & \multirow{2}{*}{$0.84-0.93$} \\
\hline & A & $5.40 \mathrm{~s}$ & & 0.42 & 0.9954 (step 2) & \\
\hline
\end{tabular}

\subsubsection{Optimal designs for estimation efficiency and detection power}

The optimal design for detection power was obtained assuming the canonical hemodynamic response function in Eq. (4.1) $\left(a_{1}=5, b_{1}=1, c=6, a_{2}=15, b_{2}=1\right.$ in Eq. (4.2)) with duration of $32 \mathrm{~s}$ and maximizing the $D$-optimality criterion $1 / \operatorname{Var}(\hat{\beta})$. The optimal design for estimation efficiency was obtained by maximizing the $D$-optimality criterion $\operatorname{det}(\operatorname{Cov}(\hat{h}))^{-1 / k}$ where $\hat{h}$ is the GLS estimator for $h$, the hemodynamic response vector sampled at $k=32 / 0.5+1=65$ time points. The entries of $h$ represent the height of the HRF at the $k$ time points. The model for estimation efficiency is obtained by replacing $h(\theta) \beta$ in Eq. (4.1) with the vector $h$. In comparison to $h(\theta) \beta, h$ can result in more flexible forms of the HRF and does not depend on the double gamma parameter vector $\theta$. The parameters $\rho$, TR, ISI and the number of events $N$ had the same values as in the calculation for the locally optimal designs and maximin designs.

We determined the optimal designs for estimation efficiency $\mathrm{Opt}_{\text {est }}$ and detection power $\mathrm{Opt}_{\text {det }}$ by the genetic algorithm. The optimal designs can be seen in Figures $4.3 \mathrm{~g}$ and $4.3 \mathrm{~h}$ and their properties are given in Table 4.5. As expected, the optimal design for estimation efficiency is an event-related design whereas the optimal design for detection power is a blocked design. Figure $4.2 \mathrm{~d}$ shows the relative efficiencies versus the $\mathrm{MMD}_{\mathrm{GA}}$ at the different expansion points. These relative efficiencies are based on Eq. (4.15) with the estimators $\hat{\tau}$ being the GLS estimators for nonlinear estimation of the double gamma function. Figure $4.2 \mathrm{~d}$ illustrates that the optimal design for estimation efficiency performs better versus the $\mathrm{MMD}_{\mathrm{GA}}$ than the optimal design for detection power. This is similar to the previous results for blocked designs and rapid event-related designs, i.e., $m$-sequences, random event-related designs with geometric or uniform distribution for the ISI. In Figure 4.2 it is 
also seen that the maximin blocked design has lower relative efficiencies than the maximin $m$-sequence, maximin geometric ISI design or maximin uniform ISI design.

When determining the relative efficiency of the $\mathrm{MMD}_{\mathrm{GA}}$ versus the optimal designs for estimation efficiency and detection power, it is seen in Table 4.5 that in general the maximin design $\mathrm{MMD}_{\mathrm{GA}}$ is not very efficient for estimation efficiency and detection power. These relative efficiencies are based on Eq. (4.15) with GLS estimators for an estimation efficiency model, i.e., $\hat{\tau}$ is replaced by $\hat{h}$, or detection power model, i.e., $\hat{\tau}$ is replaced by $\hat{\beta}$. By optimizing for the nonlinear parameters in the HRF and the amplitude $\beta$ simultaneously, the ability of the maximin design $\mathrm{MMD}_{\mathrm{GA}}$ to detect only activation, that is to estimate only the parameter $\beta$ efficiently, is considerably reduced. Although optimal designs for estimation efficiency, e.g., $m$-sequences and $\mathrm{Opt}_{\text {est }}$, had a high efficiency versus the $\mathrm{MMD}_{\mathrm{GA}}$ for the model in Eq. (4.6) and were thus efficient to estimate the nonlinear parameters and the amplitude $\beta$, the design $\mathrm{MMD}_{\mathrm{GA}}$ cannot be recommended for linear HRF estimation.

Comparing Table 4.3 with Table 4.5, it is seen that the mean block length and the mean ISI of the maximin design $\mathrm{MMD}_{\mathrm{GA}}$ are between those of the designs $\mathrm{Opt}_{\text {est }}$ and $\mathrm{Opt}_{\mathrm{det}}$. The maximin design $\mathrm{MMD}_{\mathrm{GA}}$ is thus more blocked than the design Opt $\mathrm{p}_{\text {est }}$ but its mean block length is still closer to the mean block length of Opt est $_{\text {than }}$ to the mean block length of $\mathrm{Opt}_{\mathrm{det}}$. Thus, $\mathrm{MMD}_{\mathrm{GA}}$ is only slightly more blocked than $\mathrm{Opt}_{\mathrm{est}}$.

Table 4.5: Optimal designs $\mathrm{Opt}_{\text {est }}$ and $\mathrm{Opt}_{\text {det }}$ for estimation efficiency and detection power and their relative efficiencies $(\mathrm{RE})$ in comparison with $\mathrm{MMD}_{\mathrm{GA}}$. The relative efficiencies of the optimal designs versus $\mathrm{MMD}_{\mathrm{GA}}$ are based on the model in Eq. (4.6) while the relative efficiencies of $\mathrm{MMD}_{\mathrm{GA}}$ versus the optimal designs are based on a model for estimation efficiency or detection power. Letter A stands for the stimulus and 0 represents null events.

\begin{tabular}{|c|c|c|c|c|c|c|}
\hline & & Mean BL & Mean ISI & Frequency & $\begin{array}{l}\mathrm{RE} \text { versus } \\
\mathrm{MMD}_{\mathrm{GA}}\end{array}$ & $\begin{array}{c}\mathrm{RE} \text { of } \mathrm{MMD}_{\mathrm{GA}} \\
\text { versus optimal } \\
\text { design }\end{array}$ \\
\hline \multirow{2}{*}{$\mathrm{Opt}_{\mathrm{est}}$} & 0 & $4.21 \mathrm{~s}$ & \multirow{2}{*}{$4.63 \mathrm{~s}$} & 0.46 & \multirow{2}{*}{$0.73-0.97$} & \multirow{2}{*}{0.54} \\
\hline & A & $4.93 \mathrm{~s}$ & & 0.54 & & \\
\hline \multirow{2}{*}{$\mathrm{Opt}_{\mathrm{det}}$} & 0 & $15.50 \mathrm{~s}$ & \multirow{2}{*}{$4.88 \mathrm{~s}$} & 0.48 & \multirow{2}{*}{$0.49-0.57$} & \multirow{2}{*}{0.62} \\
\hline & A & $15.00 \mathrm{~s}$ & & 0.52 & & \\
\hline
\end{tabular}

\subsection{Discussion}

The general advantage of using a maximin design for nonlinear models is that the maximin criterion chooses a design which performs well within the range of possible values for the unknown parameters. The unknown parameters in our study were the parameters of the double gamma function. The main result was that the maximin design based on the genetic algorithm performed best. However, due to less computation time maximin designs based on $m$-sequences, random event-related designs with a uniform or geometric distribution for ISI or even an optimal design for estimation efficiency can be recommended as an alternative to a maximin design obtained by the genetic algorithm. It was furthermore 
found that the maximin design $\mathrm{MMD}_{\mathrm{GA}}$, the most efficient design, is between a blocked design and a rapid event-related design. In the following we will discuss the results per design types and finalize this discussion with the properties of an optimal design for the nonlinear model.

Buračas \& Boynton (2002) showed that $m$-sequences performed in general better for estimation efficiency than randomly generated sequences, and Kao et al. (2009) found that for estimation efficiency the design obtained by the GA was more efficient than $m$ sequences. The results of both papers are based on a linear model to estimate the HRF time points and are thus not directly comparable to our results from a model to estimate the amplitude of the HRF and the nonlinear parameters of the double gamma function. However, some similarities can be found between our results and their results. Under the assumption of empirically estimated correlated noise and for one stimulus type, Buračas \& Boynton (2002) found randomly generated sequences which were more efficient than $m$ sequences. Similarly in our study, the best random sequence with a geometric distribution for ISI was more efficient than the best $m$-sequence when compared to the maximin design $\mathrm{MMD}_{\mathrm{GA}}$ (see Figure 4.2) but of course several realizations of the random sequences are necessary to find an efficient sequence. Furthermore, it is noticeable that in this paper and in Kao et al. (2009) $m$-sequences performed well with relative efficiencies versus designs obtained from the genetic algorithm from 0.8 to almost 1 .

The major advantage of $m$-sequences is that the computation time for calculation of $\mathrm{MMD}_{m}$ is much less than the computation time for calculation of the maximin design $\mathrm{MMD}_{\mathrm{GA}}$ based on the genetic algorithm (see Table 4.3). This advantage in computation time of the $\mathrm{MMD}_{m}$ comes along with a slightly lower efficiency of the $\mathrm{MMD}_{m}$ in comparison to the $\mathrm{MMD}_{\mathrm{GA}}$ for one stimulus type. Generally, $m$-sequences are only available for certain sequence lengths $N$ and number of stimulus types $Q$, i.e., $N+1$ and $Q+1$ have to be a prime or power of a prime. The problem of the sequence length can be handled by concatenating several $m$-sequences or truncating $m$-sequences so that the obtained sequence still maintains to a high degree desirable properties of $m$-sequences. These properties, which lead to a high efficiency of $m$-sequences especially for estimation of the HRF, are almost perfect counterbalancing, equal number of trials for different stimulus types and orthogonality of the $m$-sequence to cyclically shifted versions of itself (Buračas \& Boynton, 2002). The estimation efficiency of $m$-sequences seems to be robust to truncation (Liu, 2004). Liu (2004) also generated clustered $m$-sequences which are constructed from $m$-sequences by clustering events of the same stimulus type and provide a trade-off between estimation efficiency and detection power. The possibility of constructing efficient sequences from $m$-sequences widens the applicability of $m$-sequences. To our knowledge not much research has been done to construct alternative efficient sequences from $m$-sequences when $m$-sequences are not available for a given number of stimulus types.

Our results for the geometric distribution showed that an event occurrence probability of about $p=0.5$ was optimal. This probability is similar to previous results for estimation efficiency and detection power by Friston et al. (1999), Birn et al. (2002) and Zarahn \& Friston (2002). For the uniform distribution, a smallest ISI of $2.5 \mathrm{~s}$ together with ISIs which were $5 \mathrm{~s}$ apart from each other, i.e., $2.5 \mathrm{~s}, 7.5 \mathrm{~s}, 12.5 \mathrm{~s}, 17.5 \mathrm{~s}$, proved to be efficient, 
and the maximin design had ISIs $2.5 \mathrm{~s}, 7.5 \mathrm{~s}$ and $12.5 \mathrm{~s}$ for linearity as well as nonlinearity. The maximin designs $\mathrm{MMD}_{\text {geo }}$ and $\mathrm{MMD}_{\text {uni }}$ are useful because they have a high relative efficiency, needed less computation time than the maximin design $\mathrm{MMD}_{\mathrm{GA}}$ in our situation (see Table 4.3) and they are above all available for any number of stimulus types in contrast to $m$-sequences. However, it is necessary to simulate several random sequences for the maximin design $\mathrm{MMD}_{\text {geo }}$ or $\mathrm{MMD}_{\text {uni }}$ and choose the best one among these sequences in terms of its minimum relative efficiency as not any randomly generated sequence might be efficient. The least efficient designs were the maximin blocked design and the maximin constant ISI design. The maximin blocked design had a block length of $25 \mathrm{~s}$ for linearity and a block length of $22.5 \mathrm{~s}$ for nonlinearity. To avoid nonlinearity completely and if one wants to use a constant ISI design, the maximin constant ISI design with $17.5 \mathrm{~s}$ can be recommended.

Optimal designs for estimation and detection power were studied in Section 4.4.3 to show how much efficiency is lost by choosing the incorrect model and optimization criterion for the final data analysis. It was seen that optimal designs for one purpose might not be efficient for another purpose. For example, the optimal design for detection power $\mathrm{Opt}_{\text {det }}$ had a medium relative efficiency compared to the maximin design $\mathrm{MMD}_{\mathrm{GA}}$ based on the nonlinear model. In addition, the maximin design $\mathrm{MMD}_{\mathrm{GA}}$ did not perform well for estimation efficiency or detection power. It is thus relevant to specify the model and the optimization criterion in consistency with the final analysis.

The properties of the optimal design for nonlinear estimation can be determined from the most efficient and most flexible design $\mathrm{MMD}_{\mathrm{GA}}$. The maximin design $\mathrm{MMD}_{\mathrm{GA}}$ had a stimulus frequency close to 0.5 which was also the optimal stimulus frequency for the geometric distribution under linearity. It can thus be concluded that for one stimulus type and under linearity of the HRF the optimal stimulus frequency for the design for nonlinear estimation is 0.5 or a close value. This frequency 0.5 equals the $D$-optimal frequency for detection power and estimation efficiency $p_{D}=1 /(Q+1)$ for $Q=1$ and uncorrelated errors (Maus et al., 2010b). Under linearity of the HRF, a mean ISI of $5 \mathrm{~s}$ can be recommended while under nonlinearity the mean ISI should be slightly higher.

Furthermore, a mixture of a blocked and a rapid event-related design seems to be the optimal design for nonlinear estimation. The explanation for this might be that the nonlinear parameters of the double gamma function push the optimal design into another direction than the amplitude parameter $\beta$. The optimal estimation for the nonlinear double gamma parameters and the optimal estimation of the second till sixth entry in $\tau$, which are products of the nonlinear parameters with the amplitude parameter $\beta$, may demand a rapid event-related design. In contrast, the first parameter of $\tau$ in Eq. (4.6), the amplitude parameter $\beta$, may be efficiently estimated by a blocked design.

One restriction of our study could be that a limited range for the HRF parameters was considered. The high minimum relative efficiencies above 0.8 of the locally optimal designs obtained by the genetic algorithm could be due to the limited range for the HRF parameters. Additional numerical calculations were performed with a broader range of the HRF parameters $a_{1}$ and $b_{1}$, while the parameters for the undershoot were set to the canonical value of $a_{2}=15, b_{2}=1$ and $c=6$. Moreover, results were obtained for a broader 
range of the parameters for the undershoot with the parameters of the peak $a_{1}$ and $b_{1}$ fixed to a standard value of $a_{1}=5$ and $b_{1}=1$. For these two parameter spaces, the minimum relative efficiencies of the locally optimal designs obtained by the genetic algorithm were all above 0.78 which is similar to the results in Section 4.4.1.1 for the limited simultaneous parameter space of all HRF parameters.

Another restriction might be that we considered only one stimulus type, and that the results may not extend to multiple trial types. However, to estimate the subject-specific parameters of a nonlinear HRF model like the double gamma function, it would be useful to perform a run of a simple design with only one stimulus type. From this run, the active voxels or regions can be localized and the HRF parameters can be estimated such that they can be used for further data analyses from experiments with more stimulus types. Our code for the genetic algorithm can also be used for multiple trial types assuming that the hemodynamic response functions of these different trial types have the same double gamma function parameters and differ only in amplitude. In the following, we will discuss which results are expected to be valid for more than one stimulus type. Further work is nevertheless needed to draw firm conclusions on optimal designs with multiple trial types for nonlinear estimation of the HRF.

The best maximin design seems to be a mixture of a blocked and a rapid event-related design, and the optimal trade-off between "blockiness" and "event-relatedness" can best be achieved by an open search in the design space as performed by the genetic algorithm. As a consequence, we expect that the following order of efficiency from most efficient to least efficient will extend to multiple trial types: $\mathrm{MMD}_{\mathrm{GA}}, \mathrm{MMD}_{\mathrm{m}} / \mathrm{MMD}_{\text {geo }} / \mathrm{MMD}_{\text {uni }} / \mathrm{Opt}_{\mathrm{est}}$, $\mathrm{MMD}_{\mathrm{B}} / \mathrm{Opt}_{\mathrm{det}}, \mathrm{MMD}_{\mathrm{CI}}$. The computation time for the maximin designs is expected to be from highest to lowest: $\mathrm{MMD}_{\mathrm{GA}}, \mathrm{MMD}_{\text {geo }} / \mathrm{MMD}_{\mathrm{uni}}, \mathrm{MMD}_{\mathrm{m}} / \mathrm{MMD}_{\mathrm{B}} / \mathrm{MMD}_{\mathrm{CI}}$.

The optimal stimulus frequency in this paper was close to $1 /(Q+1)$ and may extend to higher number of stimulus types $Q$. The reason for this extension is that the optimality criterion for nonlinear estimation favors a mixture of a blocked design and a rapid eventrelated design and seems thus to behave like a multi-objective criterion combining detection power and estimation efficiency (Wager \& Nichols, 2003; Kao et al., 2009). The $D$-optimal stimulus frequency for detection power and for estimation efficiency is $1 /(Q+1)$ and a combined criterion should have the same $D$-optimal frequency (Maus et al., 2010b). Furthermore, as the $\mathrm{MMD}_{\mathrm{GA}}$ is presumed to be between a blocked design and a rapid event-related design, the mean block length of the maximin design $\mathrm{MMD}_{\mathrm{GA}}$ is expected to be between the mean block length of the optimal design for estimation efficiency and detection power.

We presented a common method in optimal design theory to determine optimal designs for nonlinear models. A Taylor approximation was used to linearize the nonlinear model and the maximin design was chosen from the locally $D$-optimal designs. This method can be used to perform further research on optimal designs for estimation of the nonlinear parameters of the double gamma function or parameters of other nonlinear models in fMRI data analysis. 


\section{CHAPTER 5}

\section{Optimal design of multi-subject blocked fMRI experiments}

This chapter will be published in NeuroImage (article in press) with co-authors Gerard J. P. van Breukelen, Rainer Goebel and Martijn P. F. Berger. 


\begin{abstract}
The design of a multi-subject fMRI experiment needs specification of the number of subjects and scanning time per subject. For example, for a blocked design with conditions $A$ or $B$, fixed block length and block order $A B N$, where $N$ denotes a null block, the optimal number of cycles of $A B N$ and the optimal number of subjects have to be determined. This paper presents a method to determine the optimal number of subjects and optimal number of cycles for a blocked design based on the $A$-optimality criterion and a linear cost function by which the number of cycles and the number of subjects are restricted. Estimation of individual stimulus effects and estimation of contrasts between stimulus effects are both considered. The mixed-effects model is applied and analytical results for the $A$-optimal number of subjects and $A$-optimal number of cycles are obtained under the assumption of uncorrelated errors. For correlated errors with AR1 error structure, numerical results are presented. Our results show how the optimal number of cycles and subjects depend on the within- to between-subject variance ratio. Our method is a new approach to determine the optimal scanning time and optimal number of subjects for a multi-subject fMRI experiment. In contrast to previous results based on power analyses, the optimal number of cycles and subjects can be described analytically and costs are considered.
\end{abstract}

Keywords: optimal design, blocked design, number of subjects, number of cycles, cost function 


\subsection{Introduction}

There exists a trade-off between number of subjects and number of measurements in time per subject in terms of statistical efficiency and experimental costs in any kind of experiment. Statistical efficiency increases with increasing number of subjects and increasing number of measurements in time per subject, but the same holds for experimental costs. Worsley et al. (2002) pointed out that if the variance between subjects is higher than the variance within subjects, it is better to scan more subjects with a shorter scanning time per subject than to scan fewer subjects with a longer scanning time per subject. However, Worsley et al. (2002) concluded that due to additional costs per subject a compromise between number of subjects and scanning time has to be chosen. This paper presents a method which will be useful for fMRI researchers in choosing the optimal number of subjects and scanning time. Given the high costs for fMRI experiments, such a method is relevant for these experiments.

Generally, the optimization of fMRI experiments has been studied for single-subject fMRI experiments while less attention has been given to the optimization of multi-subject fMRI experiments. For single-subject fMRI experiments, it has been shown that blocked designs are statistically optimal for detection of stimulus related activation, called detection power, whereas event-related designs are statistically optimal for estimation of the hemodynamic response function (HRF), called estimation efficiency. Bianciardi et al. (2004) studied the effect of filtering and experimental design on second-level group analyses. Furthermore, the statistical power for multi-subject fMRI experiments was studied and sample size recommendations based on power calculations were given by Desmond \& Glover (2002), Hayasaka et al. (2007), Mumford \& Nichols (2008) and Murphy \& Garavan (2004). In this paper, a new approach is given for the optimization of a multi-subject fMRI experiment, taking into account the costs per individual subjects, for scanning time and for the total experiment by a linear cost function.

This paper focuses on the $A$-optimality criterion (Atkinson et al., 2007; Dale, 1999; Liu et al., 2001) which selects as optimal the design for which the sum of the variances of the estimators for the unknown group parameters is minimized. A linear mixed-effects model is used to describe the relationship between unknown parameters and the fMRI signal. This type of model is often also referred to as random-effects model in fMRI literature (Penny \& Holmes, 2004). The number of subjects and the scanning time are restricted by a linear cost function describing the experimental costs due to the number of subjects and scanning time. For a blocked design with task blocks $A_{1}, \ldots, A_{Q}$, with null blocks denoted by $N$ and the block order $A_{1} \ldots A_{Q} N$, the scanning time depends on the number of cycles of $A_{1} \ldots A_{Q} N$, the length of stimulus blocks and null blocks and the number of stimulus types $Q$. Assuming uncorrelated errors, we derived analytically the optimal number of subjects and number of cycles for a blocked design assuming fixed block lengths and fixed number of stimulus types. For a linear mixed-effects model with an AR1 error structure (Bullmore et al., 1996; Chatfield, 2004), numerical calculations were performed to find the optimal number of subjects and cycles. The optimal number of subjects and cycles were found to depend on the ratio of within- to between-subject variance for uncorrelated errors as well as correlated errors. Furthermore for both error structures, the ratio of costs per 
subject and costs per scanning time is crucial for the optimal choice of number of subjects and cycles. The results are valid for any number of stimulus type.

The outline of this paper is as follows. In Section 5.2 the linear mixed-effects model, the $A$-optimality criterion and the cost function are presented and the numerical calculations for correlated errors are explained. In Section 5.3.1 the analytical results for the optimal number of subjects and cycles assuming uncorrelated errors and the results of the numerical calculations for correlated errors are given. Further details on the derivations are provided in the Appendices 5.A and 5.B. The consequences of the analytical derivations and numerical calculations are illustrated by examples with common values for fMRI experiments in Section 5.3.1. Furthermore, we study in Section 5.3.2 the effect on design efficiency when the number of cycles and subjects vary slightly from the optimal design. In Section 5.4 the results are discussed and conclusions are drawn based on the results.

\subsection{Methodology}

\subsubsection{Model and estimators}

Each subject is measured at $N_{T}$ time points with a given repetition time (TR) and the data vector $Y_{i}$ is obtained for each subject from these measurements. It is assumed that $Q$ different stimulus types are presented in blocks to each subject. One block contains trials of the same stimulus type. The following first-level model is considered for subject $i$ :

$$
Y_{i}=Z \beta_{i}+S \gamma_{i}+\epsilon_{i}
$$

where $Z$ of dimension $N_{T} \times Q$ models the hemodynamic responses to the $Q$ different stimulus types and $S$ of dimension $N_{T} \times r$ models $r$ nuisance terms, e.g., intercept, linear or quadratic trends. The errors $\epsilon_{i}$ are assumed to be normally distributed with expectation zero and covariance matrix $\sigma_{\epsilon}^{2} \Sigma$. It is assumed that all subjects have the same error covariance matrix $\sigma_{\epsilon}^{2} \Sigma$.

The interest at the first level is in estimation of the stimulus effects $\beta_{i}$. The generalized least squares (GLS) estimator for $\left(\begin{array}{ll}\beta_{i}^{T} & \gamma_{i}^{T}\end{array}\right)^{T}$ is given by

$$
\widehat{\left(\begin{array}{c}
\beta_{i} \\
\gamma_{i}
\end{array}\right)}=\left(\left[\begin{array}{ll}
Z & S
\end{array}\right]^{T} \Sigma^{-1}\left[\begin{array}{ll}
Z & S
\end{array}\right]\right)^{-1}\left[\begin{array}{ll}
Z & S
\end{array}\right]^{T} \Sigma^{-1} Y_{i}
$$

The matrix $[Z S]$ is a concatenation of the matrices $Z$ and $S$. The generalized least squares (GLS) estimator of $\hat{\beta}_{i}$ in Eq. (5.2) is given by

$$
\hat{\beta}_{i}=\left(Z^{T} V^{T}\left(I-P_{V S}\right) V Z\right)^{-1} Z^{T} V^{T}\left(I-P_{V S}\right) V Y_{i},
$$

where $V=\Sigma^{-1 / 2}$ is the inverse square root matrix of the error correlation matrix $\Sigma$ and $P_{V S}=V S\left(S^{T} \Sigma^{-1} S\right)^{-1} S^{T} V^{T}$ is the projection matrix onto the space spanned by the columns of the matrix VS (column space) (Liu \& Frank, 2004; Lovell, 1963; Seber, 1977). The projection matrix $P_{V S}$ is needed to obtain a closed form expression for $\hat{\beta}_{i}$. The covariance matrix of $\hat{\beta}_{i}$ is given by

$$
\operatorname{Cov}\left(\hat{\beta}_{i}\right)=\sigma_{\epsilon}^{2}\left(Z^{T} V^{T}\left(I-P_{V S}\right) V Z\right)^{-1} .
$$


We define the detrended and decorrelated design matrix $Z^{*}=\left(I-P_{V S}\right) V Z$, which is the decorrelated design matrix $V Z$ after removal of decorrelated trends, e.g., constant, linear and quadratic trends, by application of the projection matrix $\left(I-P_{V S}\right)$. The following equations are then obtained:

$$
\begin{aligned}
\hat{\beta}_{i} & =\left(Z^{* T} Z^{*}\right)^{-1} Z^{* T} V Y_{i} \\
\operatorname{Cov}\left(\hat{\beta}_{i}\right) & =\sigma_{\epsilon}^{2}\left(Z^{* T} Z^{*}\right)^{-1} .
\end{aligned}
$$

The matrix $\left(Z^{* T} Z^{*}\right) / \sigma_{\epsilon}^{2}$ is the Fisher information matrix which can be used to measure the amount of information present in the data about the unknown parameters (Liu \& Frank, 2004) and to determine the optimal design (Atkinson et al., 2007).

The second-level model is given by

$$
\beta_{i}=\beta_{G}+b_{i}
$$

where $\beta_{G}$ are the group stimulus effects and $b_{i}$ are the random stimulus effects for subject $i$. The random effects $b_{i}$ are assumed to be normally distributed with expectation 0 and covariance matrix $\sigma_{q}^{2} D$. The matrix $D$ is the correlation matrix of the random-effects vector $b_{i}$. All random effects in the random-effects vector $b_{i}$ are assumed to have the same random-effects variance, also called between-subject variance, $\sigma_{q}^{2}$. Combining Eq. (5.1) with Eq. (5.7) gives the following model:

$$
Y_{i}=Z \beta_{G}+Z b_{i}+S \gamma_{i}+\epsilon_{i}
$$

The covariance matrix of the GLS estimator $\hat{\beta}_{G}$ for $\beta_{G}$ in Eq. (5.8) is given by

$$
\begin{aligned}
\operatorname{Cov}\left(\hat{\beta}_{G}\right) & =\frac{1}{N}\left(\sigma_{\epsilon}^{2}\left(Z^{T} V^{T}\left(I-P_{V S}\right) V Z\right)^{-1}+\sigma_{q}^{2} D\right), \\
& =\frac{1}{N}\left(\sigma_{\epsilon}^{2}\left(Z^{* T} Z^{*}\right)^{-1}+\sigma_{q}^{2} D\right),
\end{aligned}
$$

where $N$ is the number of subjects. The term $\sigma_{\epsilon}^{2}\left(Z^{* T} Z^{*}\right)^{-1}$ is the within-subject covariance matrix of $\hat{\beta}_{i}$ assuming the same error covariance $\sigma_{\epsilon}^{2} \Sigma$ for all subjects, and $\sigma_{q}^{2} D$ is the between-subject covariance matrix of the stimulus effects. Equation (5.9) can be seen from Section 4 in Beckmann et al. (2001).

\subsubsection{Optimality criterion and relative efficiency}

Several optimality criteria exist, each with their own advantages and disadvantages (Atkinson et al., 2007). The two mainly applied criteria are the $A$ - and $D$-optimality criteria. In this paper, the $A$-optimality criterion is applied and the number of cycles and the number of subjects are optimized subject to a linear cost function given in Section 5.2.3. The $A$-optimality criterion has been applied before for optimization of single-subject fMRI experiments by Dale (1999), Liu et al. (2001) and Wager \& Nichols (2003). Assuming equal frequency for all $Q$ stimulus types, Liu \& Frank (2004) showed that the $A$-optimal stimulus frequency for estimation of stimulus effects is equal to $p=1 /(Q+\sqrt{Q})$. For a blocked 
design, this will result in longer null blocks than stimulus blocks. For estimation of pairwise contrasts, $p=1 / Q$ is the optimal stimulus frequency and results in no null blocks.

The $D$-optimality criterion minimizes the simultaneous confidence ellipsoid of the estimators for the effects of interest whereas the $A$-optimality criterion minimizes the sum of the variances of the estimators. This renders the $A$-optimality criterion more easy to understand for practitioners. One strength of the $A$-optimality criterion in our specific example is the possibility to derive analytical results. Another strength is the fact that off-diagonal elements of the random-effects covariance matrix $\sigma_{q}^{2} D$ do not have to be taken into account when considering individual stimulus effects by the $A$-optimality criterion. One general advantage of the $D$-optimality criterion is that the $D$-optimal design is unaffected by linear transformation of the design matrix $Z$, i.e., linear transformation of the columns of the design matrix, whereas this is generally not the case for the $A$-optimality criterion. However, no linear transformations of the design matrix $Z$ seem to be relevant for the present model except scaling of the HRF which would not affect the $A$-optimal design.

We will compare blocked designs with block order $A_{1} \ldots A_{Q} N$ which means that a block of stimulus type $A_{1}$ is followed by a block of stimulus type $A_{2}$ etc. Finally, $A_{Q}$ is followed by a null block denoted by $N$. This block order has been shown to be statistically optimal for estimation of individual stimulus effects (Maus et al., 2010a). By assuming null block length equal to $0 \mathrm{~s}$, block order $A_{1} \ldots A_{Q}$ is obtained which is efficient for estimation of pairwise contrasts. A cycle $A_{1} \ldots A_{Q} N$, consisting of the individual stimulus blocks and a null block, can be repeated several times for the considered blocked designs. The compared blocked designs differ in the number of cycles $N_{C}$ and number of subjects $N$. The number of stimulus types and block lengths are not optimized as these parameters will be fixed by the researchers for his or her specific experiment. Furthermore, optimal values for block length have already been given in literature (Aguirre \& D'Esposito, 1999; Chein \& Schneider, 2003; Maus et al., 2010a,b). This optimal value will be valid for all scanning times as the optimal value is influenced by the power spectrum of the HRF and the power spectrum of the error noise (Aguirre \& D'Esposito, 1999).

Thus for fixed block lengths, stimulus onset asynchrony (SOA) and number of stimulus types, the design space $\Xi$, from which the optimal design will be chosen, consists of these blocked designs $\xi$ with $N_{C}$ cycles and $N$ subjects:

$$
\begin{aligned}
\Xi= & \left\{\xi \mid \xi \text { is a blocked design with block order } A_{1} \ldots A_{Q} N,\right. \\
& \left.N_{C} \text { cycles and } N \text { subjects, } N_{C}, N \in \mathbb{N}\right\} .
\end{aligned}
$$

The stimulus onset asynchrony (SOA) refers here to the time between successive trials in a stimulus block or null events in a null block.

Estimation of all individual stimulus effects $\beta_{G}$ and estimation of stimulus effects contrasts $C \beta_{G}$ with $c \times Q$ contrast matrix $C$ are considered. The contrast matrix $C$ indicates $c$ contrasts of interest. The $A$-optimal design minimizes the trace of the covariance matrix $\operatorname{Cov}\left(C \hat{\beta}_{G}\right)$ over all possible designs in the design space. This $\operatorname{trace}\left(\operatorname{Cov}\left(C \hat{\beta}_{G}\right)\right)$ will vary for designs with different number of cycles and subjects. We denote by $C \hat{\beta}_{G}^{\xi}$ the estimator obtained for design $\xi$. The trace of $\operatorname{Cov}\left(C \hat{\beta}_{G}^{\xi}\right)$ is equal to the sum of the variances for 
each contrast in $C \hat{\beta}_{G}$ and gives a measure of how precisely these contrasts (or individual stimulus effects if $C$ is an identity matrix) are estimated. It follows from Eq. (5.9) that the $A$-optimal design minimizes

$$
\operatorname{trace}\left(\operatorname{Cov}\left(C \hat{\beta}_{G}^{\xi}\right)\right)=\frac{\sigma_{q}^{2}}{N}\left(\frac{\sigma_{\epsilon}^{2}}{\sigma_{q}^{2}} \operatorname{trace}\left(C\left(Z^{* T} Z^{*}\right)^{-1} C^{T}\right)+\operatorname{trace}\left(C D C^{T}\right)\right)
$$

over all designs $\xi$ in $\Xi$ subject to the linear cost function described in the next Section 5.2.3. We can simplify Eq. (5.11) for uncorrelated errors, SOA equal to TR and a nuisance matrix $S$ equal to $1_{N_{T}}$, where $1_{N_{T}}$ is a vector with $N_{T} 1$ 's as entries and models an intercept. In Appendix 5.A it is shown the matrix $Z^{* T} Z^{*}$ can then be expressed as $N_{C} \cdot M$, where $N_{C}$ is the number of cycles of $A_{1} \ldots A_{Q} N$ and $M / \sigma_{\epsilon}^{2}$ is the $Q \times Q$ information matrix of one cycle $A_{1} \ldots A_{Q} N$. Thus, the trace of the covariance matrix of $C \hat{\beta}_{G}$ can be rewritten for uncorrelated error as

$$
\operatorname{trace}\left(\operatorname{Cov}\left(C \hat{\beta}_{G}\right)\right)=\frac{\sigma_{q}^{2}}{N}\left(\frac{\sigma_{\epsilon}^{2}}{\sigma_{q}^{2}} \frac{1}{N_{C}} \cdot \operatorname{trace}\left(C M^{-1} C^{T}\right)+\operatorname{trace}\left(C D C^{T}\right)\right) .
$$

The matrix $M$ is only invertible when null blocks are included in the design (see Appendix 5.A for further explanation), but the general expression in Eq. (5.11) can always be used. From Eqs. (5.11) and (5.12) it can be seen that the optimal number of subjects $N$ and the optimal number of cycles $N_{C}$, which minimize the $A$-optimality criterion, depend on the variance ratio $\sigma_{\epsilon}^{2} / \sigma_{q}^{2}$, but not on the specific values for $\sigma_{\epsilon}^{2}$ or $\sigma_{q}^{2}$.

The relative efficiency, which can be used to compare a design $\xi$ with the optimal design $\xi^{*}$, is given by the following ratio

$$
\operatorname{RE}\left(\xi \mid \xi^{*}\right)=\frac{\operatorname{trace}\left(\operatorname{Cov}\left(C \hat{\beta}_{G}^{\xi^{*}}\right)\right)}{\operatorname{trace}\left(\operatorname{Cov}\left(C \hat{\beta}_{G}^{\xi}\right)\right)}
$$

The relative efficiency is a value between 0 and 1 with values close to 0 indicating a very low efficiency of the design $\xi$ versus the optimal design $\xi^{*}$ and values close to 1 indicating a high efficiency of the design $\xi$ versus the optimal design $\xi^{*}$. The inverse of the relative efficiency $\operatorname{RE}\left(\xi \mid \xi^{*}\right)$ gives the number of times that design $\xi$ has to be repeated to be as efficient as the optimal design $\xi^{*}$, i.e., to have the same precision for estimation of $C \beta_{G}$ and the same power if $C \beta_{G}$ is a contrast or single stimulus effect.

\subsubsection{Cost function}

A cost function for a blocked fMRI experiment with one run and one session is considered. For the cost function we are denoting the total costs of the experiment with $C_{T}$, the costs per subjects with $C_{1}$ and the costs per unit scanning time $T_{S}$ with $C_{2}$. $C_{1}$ includes the subject fee, recruitment costs and equipment per subject, and costs due to preparation of a subject or the scanner before the subject's session. The costs of $C_{1}$ might rise if a special population is considered, e.g., subjects with a rare illness. It should be noted that the value associated with $C_{2}$ depends on the unit of time used, e.g., $C_{2}=€ 400 / \mathrm{h} \approx € 6.66 / \mathrm{min} \approx$ $€ 0.11 / \mathrm{s}$. The effective scanning time $T_{S}$ is here the length of one run, i.e., one continuous 
period of scanning. For a blocked design with $Q$ stimulus types, the scanning time $T_{S}$ is given by

$$
T_{S}=N_{C} \cdot\left(Q \cdot N_{B L_{A}}+N_{B L_{0}}\right) \cdot \mathrm{SOA}
$$

where $N_{C}$ is the number of cycles of $A_{1} \ldots A_{Q} N, N_{B L_{A}}$ is the number of events per stimulus block for all stimulus types, $N_{B L_{0}}$ is the number of null events per null block and $Q$ is the number of stimulus types. The cost function of the whole experiment expresses the total costs $C_{T}$ as sum of the costs for all subjects and the costs for the scanner time of all subjects:

$$
C_{T}=N C_{1}+N \cdot N_{C} \cdot\left(Q \cdot N_{B L_{A}}+N_{B L_{0}}\right) \cdot \mathrm{SOA} \cdot C_{2} .
$$

Note that $C_{2}$ is given above as costs per second, i.e., costs for one second scanning time. The number of subjects $N$ and the scanning time $T_{S}$ are restricted by the cost function and the assumed total costs. As explained in Section 5.2.2 block lengths, number of stimulus types $Q$ and SOA are assumed to be fixed by the researcher. Thus, restriction of the scanning time is equivalent to restriction of the number of cycles $N_{C}$ as can be seen from Eq. (5.14). We assume fixed total costs $C_{T}$ and want to find the combination of number of subjects and scanning time $T_{S}$ which minimizes $\operatorname{trace}\left(C \hat{\beta}_{G}\right)$ in Eq. (5.11).

\subsubsection{Numerical calculations}

In the following we give an outline of the numerical calculations which were performed in MATLAB (The Mathworks Inc, Natick, MA) to find optimal designs for a model with correlated errors. The code for correlated errors is available on request from the first author. An AR1 covariance structure with AR1 autocorrelation $\rho$ was assumed for the covariance matrix $\sigma_{\epsilon}^{2} \Sigma$ of all within-subject errors $\epsilon_{i}(i=1, \ldots, N)$. The optimal number of subjects and cycles were chosen as the values which minimize Eq. (5.11) for a specified design (SOA, null block length, stimulus block length, number of stimulus types $Q$ ), contrast matrix $C$, autocorrelation $\rho$ and variance ratio $\sigma_{\epsilon}^{2} / \sigma_{q}^{2}$ of within-subject variance $\sigma_{\epsilon}^{2}$ to between-subject variance $\sigma_{q}^{2}$. As the variance $\sigma_{q}^{2}$ is the same for all designs, it does not influence the values for which the minimum of Eq. (5.11) is obtained. It is therefore sufficient to focus on the variance ratio.

The number of cycles was varied from a lower boundary to an upper boundary. If no highpass filtering was assumed, the optimal number of cycles for uncorrelated errors to be derived in Section 5.3.1.1 was chosen as a lower boundary as it was expected and confirmed by preliminary results that the optimal number of cycles for correlated errors is higher than for uncorrelated errors. The reason for this relationship is explained in Section 5.3.1.2. If highpass filtering was assumed, the lower border was set equal to 1 . The upper boundary was raised if the obtained optimal number of cycles for correlated errors turned out to be equal to the upper boundary.

The number of cycles was thus varied within a certain range and the number of subjects was calculated for each number of cycles with the cost function in Eq. (5.15) for fixed costs. The block lengths, SOA, TR, the number of stimulus types, the autocorrelation and the 
variance ratio were likewise fixed to constants, and the hemodynamic response function was given. The combination of number of cycles and subjects, for which $\operatorname{trace}\left(\operatorname{Cov}\left(\hat{\beta}_{G}\right)\right) / \sigma_{q}^{2}$ as given by Eq. (5.11) reached its minimum, was the optimal number of cycles and optimal number of subjects.

\subsection{Results}

The optimal number of subjects and cycles for uncorrelated errors are determined in Section 5.3.1.1 analytically. We present some numerical results in Section 5.3.1.1 for the derived optimal number of cycles and subjects. For correlated errors with AR1 error structure (Bullmore et al., 1996; Chatfield, 2004), a numerical calculation in MATLAB (The Mathworks INc, Natick, MA) was performed as described in Section 5.2.4 to determine the optimal number of cycles and subjects. For the numerical results for uncorrelated errors and correlated errors, the hemodynamic response function with default parameters from the MATLAB toolbox BVQXtools_v08d (Brain Innovation, Maastricht, The Netherlands) was applied, sampled with rate $2 \mathrm{~s}$ over a period of $32 \mathrm{~s}$ and scaled to have a peak amplitude of 1 . The toolbox can be downloaded for free from http://support.brainvoyager.com/availabletools/52-matlab-tools-bvxqtools.html. The default HRF has a positive peak at $5 \mathrm{~s}$, a negative peak (undershoot) at $15 \mathrm{~s}$, a positive and negative dispersion of 1 and a positiveto-negative ratio of 6 . The exact function and parameters are further described in Maus et al. (2010a). For all calculations, it is assumed that the matrix $S$ equals $1_{N_{T}}$ except in Section 5.3.1.2 when the effect of highpass filtering is studied. In Table 5.1 a list of the applied symbols in all figures is given.

For ease of interpretation the cost ratio $C_{1} / C_{2}$ is given in the figures based on $C_{2}$ being the costs per minute scanning time. Then, a cost ratio of $C_{1} / C_{2}=12$ means that a subject costs as much as 12 minutes scanning time.

Table 5.1: List of symbols

\begin{tabular}{ll}
\hline Symbol & Meaning \\
\hline$Q$ & Number of stimulus types (excluding null events) \\
$N_{B L_{0}}$ & Number of events per null block \\
$N_{B L_{A}}$ & Number of events per stimulus block \\
$C_{T}$ & Total experimental costs \\
$C_{1}$ & Costs per subject \\
$C_{2}$ & Costs per scanning time, depending on context given per h, min or s \\
$\sigma_{\epsilon}^{2}$ & Error variance (within-subject variance) \\
$\sigma_{q}^{2}$ & Random-effects variance (between-subject variance) \\
$\rho$ & Autocorrelation coefficient for AR1 error structure \\
$N$ & Number of subjects \\
$N_{C o p t}$ & Optimal number of cycles \\
$N_{\text {opt }}$ & Optimal number of subjects \\
$C$ & Contrast matrix \\
\hline
\end{tabular}




\subsubsection{Optimal design for a given budget}

\subsubsection{Uncorrelated errors}

From Eq. (5.15) it follows that the number of subjects $N$ can be expressed as the ratio of the total experimental costs and the total costs per subject. The obtained expression for $N$ can be substituted in Eq. (5.12), the trace of $\operatorname{Cov}(C \hat{\beta})$, for $N$. In the next step, the trace of $\operatorname{Cov}(C \hat{\beta})$ is minimized with respect to $N_{C}$. This leads to the following optimal number of cycles

$$
N_{\text {Copt }}=\sqrt{\frac{C_{1}}{C_{2}}} \sqrt{\frac{\sigma_{\epsilon}^{2}}{\sigma_{q}^{2}}} \sqrt{\frac{1}{\operatorname{trace}\left(C D C^{T}\right)}} \sqrt{\frac{\operatorname{trace}\left(C M^{-1} C^{T}\right)}{\operatorname{SOA}\left(N_{B L_{0}}+Q N_{B L_{A}}\right)}} .
$$

Using the expression for $N$ obtained by the cost function in Eq. (5.15) and substituting $N_{C o p t}$ for $N_{C}$ in this expression results in the following optimal number of subjects

$$
N_{o p t}=\frac{C_{T}}{C_{1}+\sqrt{C_{1} C_{2}} \sqrt{\frac{\sigma_{\epsilon}^{2}}{\sigma_{q}^{2}}} \sqrt{\frac{1}{\operatorname{trace}\left(C D C^{T}\right)}} \sqrt{\operatorname{trace}\left(C M^{-1} C^{T}\right) \operatorname{SOA}\left(N_{B L_{0}}+Q N_{B L_{A}}\right)}} .
$$

If the ratio $\sigma_{\epsilon}^{2} / \sigma_{q}^{2}$ increases, the optimal number of cycles increases, and as a consequence the optimal number of subjects decreases. Higher fixed costs $C_{1}$ per subject or lower costs $C_{2}$ per scanning time result in a higher ratio $C_{1} / C_{2}$ so that more cycles are optimal for the $A$-optimality criterion given costs $C_{T}$. Furthermore, it can be seen that the optimal number of cycles is independent of the total costs $C_{T}$. Equations (5.16) and (5.17) are comparable to results in Moerbeek et al. (2008, p. 183, Table 4.1) for optimal nested designs with regard to the effects of cost ratio, variance ratio and costs. The factors $\operatorname{trace}\left(C M^{-1} C^{T}\right)$, $N_{B L_{0}}, N_{B L_{A}}$ and SOA describe properties of fMRI experiments.

To calculate the optimal number of cycles in Eq. (5.16) and the optimal number of subjects in Eq. (5.17) for individual stimulus effects, we need to determine $\operatorname{trace}\left(M^{-1}\right)$ as $C$ equals in this case the identity matrix $I_{Q}$ of dimension $Q$. The trace of the matrix $M^{-1}$ in Eqs. (5.16) and (5.17) however depends on the block length $N_{B L_{0}}$, the stimulus frequency $p$ and the hemodynamic response function and no closed form expression is available for $\operatorname{trace}\left(M^{-1}\right)$ depending on these factors. Thus, we will replace $M^{-1}$ by an approximation to derive more direct and insightful formulae for the optimal number of cycles and subjects. It is shown in Appendix 5.A.2 that trace $\left(M^{-1}\right)$ can be approximated by

$$
\operatorname{trace}\left(M^{-1}\right) \approx \frac{Q-Q^{2} p+Q p}{p N_{B L_{0}} H}
$$

where $H=\sum_{m=1}^{N_{h}} \sum_{n=1}^{N_{h}} h_{m} h_{n}$ depends on the hemodynamic response vector $h$. The hemodynamic response vector $h$ is the hemodynamic response function sampled with rate TR. Based on the approximation of $\operatorname{trace}\left(M^{-1}\right)$ in Eq. (5.18), the following optimal number of 
cycles and optimal number of subjects are obtained for minimization of $\operatorname{trace}\left(\operatorname{Cov}\left(\hat{\beta}_{G}\right)\right)$

$$
\begin{aligned}
N_{\text {Copt }} & =\sqrt{\frac{C_{1}}{C_{2}}} \sqrt{\frac{\sigma_{\epsilon}^{2}}{\sigma_{q}^{2}}} \sqrt{\frac{(1-p Q)(1-p Q+p)}{p}} \frac{1}{\sqrt{H N_{B L 0}^{2} \mathrm{SOA}}}, \\
N_{\text {opt }} & =\frac{C_{T}}{C_{1}+\sqrt{C_{1} C_{2}} \sqrt{\frac{\sigma_{\epsilon}^{2}}{\sigma_{q}^{2}}} \sqrt{\frac{(1-p Q+p)}{p(1-p Q)}} \sqrt{\frac{\mathrm{SOA}}{H}}} .
\end{aligned}
$$

Equations (5.19) and (5.20) can be seen by substituting trace $\left(M^{-1}\right)$ with its approximation in Eq. (5.18) and by substituting $N_{B L_{A}}$ with $[p /(1-p Q)] N_{B L_{0}}$ in Eqs. (5.16) and (5.17). The equation $N_{B L_{A}}=[p /(1-p Q)] N_{B L_{0}}$ follows from the definition of the stimulus frequency $p=N_{B L_{A}} /\left(Q \cdot N_{B L_{A}}+N_{B L_{0}}\right)$. The approximation of $M^{-1}$ in Appendix 5.A.2 was tested on its precision for trace $\left(M^{-1}\right)$ and results indicate that the approximation can be imprecise for the off-diagonal elements in $M^{-1}$. These off-diagonal elements were not needed for $\operatorname{trace}\left(M^{-1}\right)$ but would be needed for $\operatorname{trace}\left(C M^{-1} C^{T}\right)$. Therefore, only approximated formulae for $N_{\text {Copt }}$ and $N_{\text {opt }}$ for the case $C=I_{Q}$ are presented.

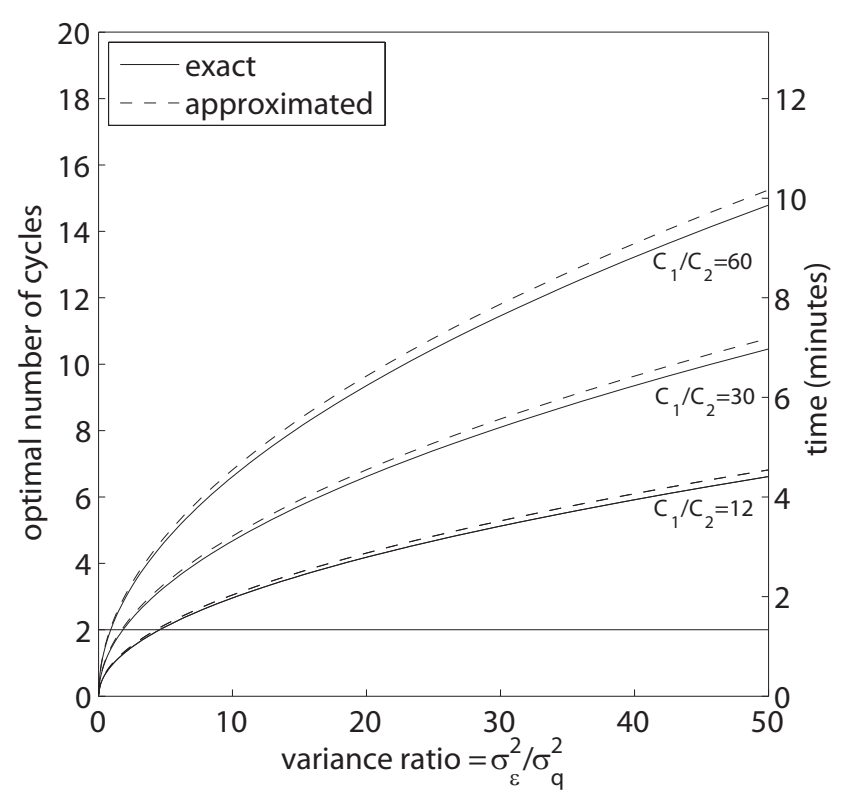

(a) Optimal number of cycles

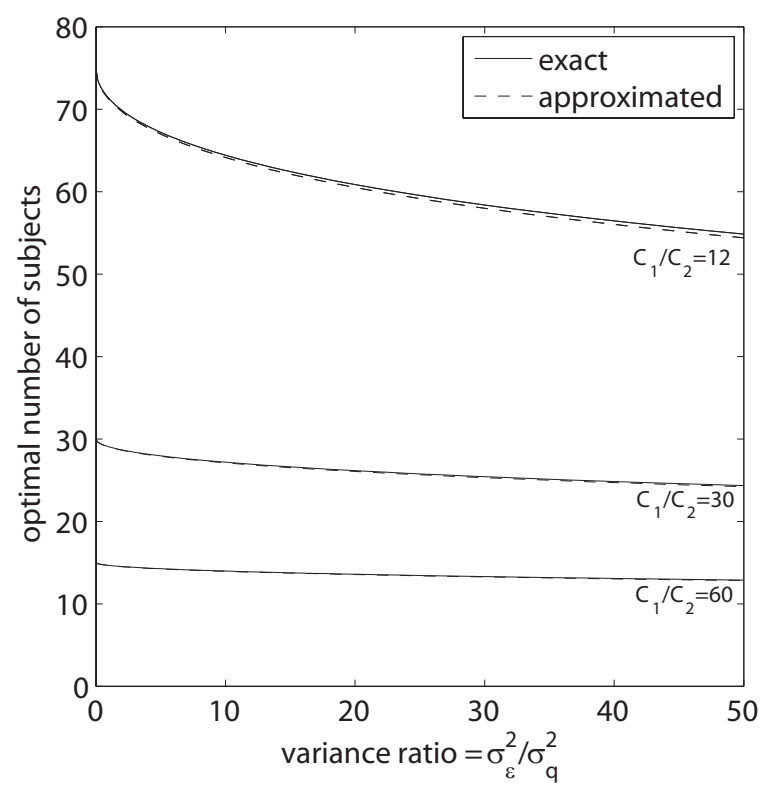

(b) Optimal number of subjects

Figure 5.1: Optimal number of cycles and subjects for uncorrelated errors, $C=I_{2}, Q=2$, $\mathrm{TR}=2 \mathrm{~s}, \mathrm{SOA}=2 \mathrm{~s}, N_{B L_{0}}=8(16 \mathrm{~s})$ and $N_{B L_{A}}=6(12 \mathrm{~s}), C_{T}=€ 6000, C_{2}=€ 400 / \mathrm{h}$ $\approx € 6.66 / \mathrm{min}$ and $C_{1}=€ 80, C_{1}=€ 200$ or $C_{1}=€ 400$. The horizontal line indicates the minimum number of cycles of 2. The exact values are based on Eqs. (5.16) and (5.17) while the approximated values are based on Eqs. (5.19) and (5.20). On the right $y$-axis for the optimal number of cycles the corresponding scanning time in minutes is given.

In Figure 5.1 the optimal number of cycles and subjects for a design example and costs example are given. The optimal estimation of two individual stimulus effects, i.e., $C=I_{2}$, is considered. The $A$-optimal stimulus frequency of $p=1 /(\sqrt{2}+2) \approx 0.30$ for estimation of stimulus effects was applied. It can be seen in Figure 5.1 that the optimal number of 
cycles and subjects based on Eqs. (5.16) and (5.17) were similar to the optimal number of cycles and subjects based on the approximation as given by Eqs. (5.19) and (5.20). The approximated results were also similar to the results based on Eqs. (5.19) and (5.20) for different costs, different number of stimulus types and stimulus frequency $p=1 /(Q+1)$ which results in stimulus block lengths equal to the null block length, while the $A$-optimal stimulus frequency results in longer block lengths for null blocks. Figure 5.1 illustrates that for increasing cost ratio the optimal number of cycles increases whereas the optimal number of subjects decreases. This is generally true for the optimal number of cycles whereas for the optimal number of subjects this relationship holds because $C_{2}$ is fixed. Furthermore, the optimal number of cycles increases and the optimal number of subject decreases for an increasing ratio of within- to between-subject variance. The described relationship between optimal number of cycles/subjects and cost ratio was also found for $p=1 /(Q+1)$. In addition, the described relationship between the optimal number of cycles/subjects and variance ratio was the same for $p=1 /(Q+1)$ as for $p=1 /(Q+\sqrt{Q})$.

The optimal number of cycles and subjects were calculated in Figure 5.1 for given total costs $C_{T}=€ 6000$, costs per scanning time $C_{2}=€ 400 / \mathrm{h} \approx € 6.66 / \mathrm{min} \approx € 0.11 / \mathrm{s}$ and varying costs per subject $C_{1}$. The optimal number of subjects and cycles were calculated based on Eqs. (5.16), (5.17), (5.19) and (5.20) for different cost ratios $C_{1} / C_{2}$ and $C_{2}$ given as costs per second, i.e., $C_{2}=€ 400 /(3600 \mathrm{~s}) \approx 0.11 / \mathrm{s}$. A design with two stimulus types, null block length equal to $16 \mathrm{~s}$ and stimulus block length equal to $12 \mathrm{~s}$ was assumed. This corresponds to the $A$-optimal stimulus frequency of $p=1 /(\sqrt{2}+2) \approx 0.30$ for estimation of stimulus effects. The variance ratio $\sigma_{\epsilon}^{2} / \sigma_{q}^{2}$ varied in a range from 0 to 50 as this covers a wide range of variance ratios. For values close to $0, \sigma_{q}^{2}$ dominates the variance ratio whereas for values close to $50, \sigma_{\epsilon}^{2}$ dominates the variance ratio.

The number of cycles should be at minimum 2 so that linear trends can be estimated. The maximum run time can be restricted by an assumed realistic maximum length time, e.g., 15 min to avoid boredom and fatigue of subjects. If the optimal number of cycles is unrealistic because the assumed realistic maximum run time is exceeded, the number of cycles should be decreased to the highest possible value for the number of cycles and the number of subjects has to be adapted according to this value for the number of cycles such that the total costs $C_{T}$ remain the same. In the given example with scanning time of up to $10 \mathrm{~min}$, we assumed the scanning time to be realistic. Generally, as the optimal number of cycles and subjects obtained by Eqs. (5.16) and (5.17) will be mostly no integer values, the optimal values have to be rounded.

\subsubsection{Correlated errors}

In Figure 5.2a the optimal number of cycles for correlated errors and uncorrelated errors is displayed for a particular design with three stimulus types, null block length equal to $14 \mathrm{~s}$ and stimulus block length equal to $10 \mathrm{~s}$ (Maus et al., 2010b). The autocorrelation $\rho$ was set to 0.3 for Figure 5.2 as this value is a middle value of autocorrelation for $\mathrm{TR}=2 \mathrm{~s}$. Integer values were used for the number of cycles in the numerical calculation for correlated errors. Therefore, the optimal number of cycles and the optimal number of subjects in Figure 5.2 change in steps. Figure 5.2 illustrates that the behavior of the optimal number of cycles 
and the optimal number of subjects as a function of the variance ratio and the cost ratio is similar for uncorrelated and correlated errors. For example, the relationship between the optimal number of cycles and the variance ratio is curvilinear (square root) for uncorrelated and correlated errors. Another example is that for uncorrelated and correlated errors the optimal number of subjects decreases with increasing variance ratio and stabilizes for high variance ratios.

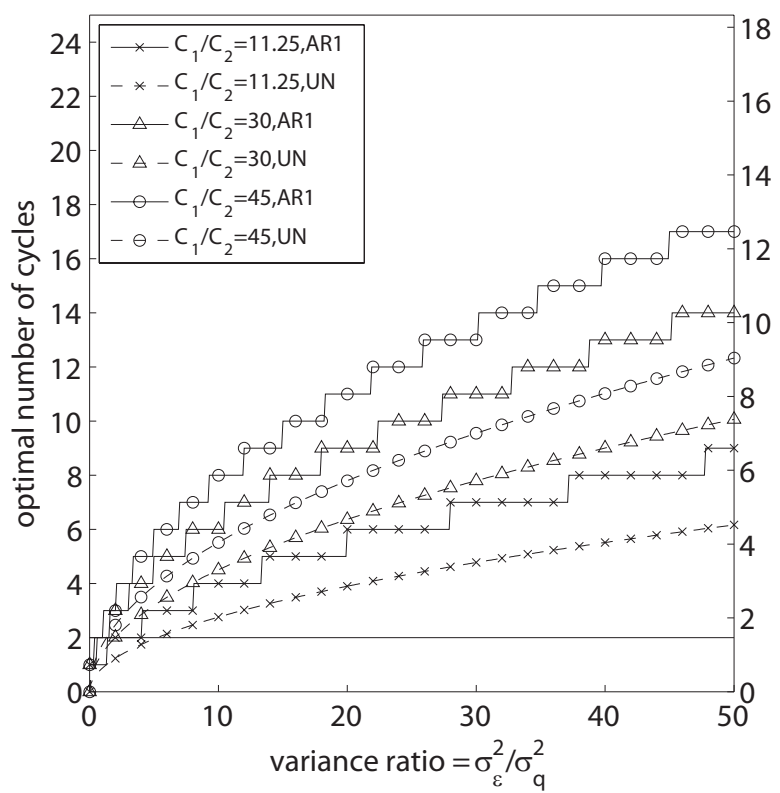

(a) Optimal number of cycles

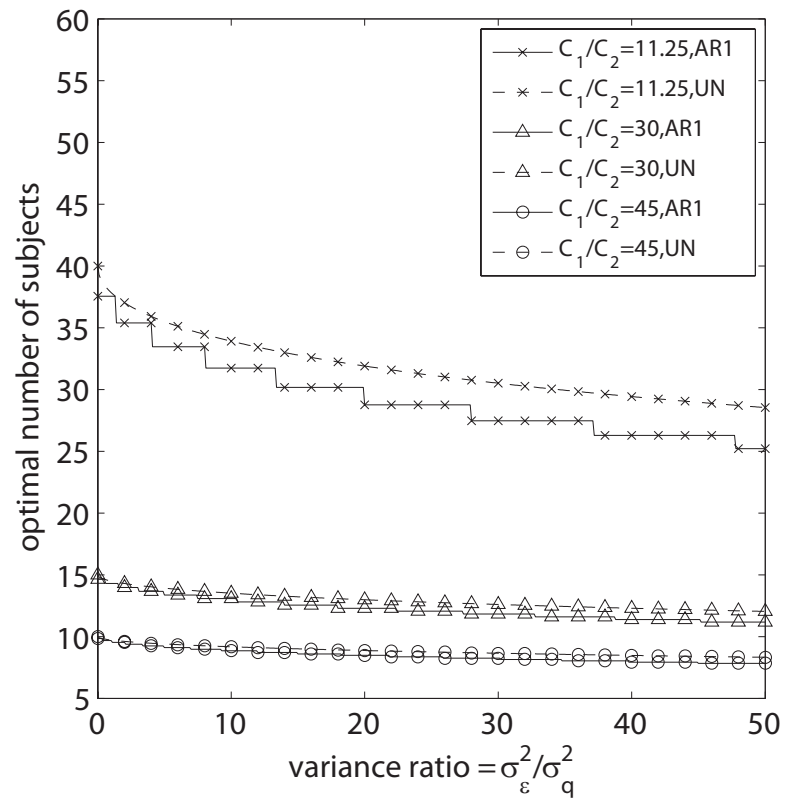

(b) Optimal number of subjects

Figure 5.2: Optimal number of cycles and subjects for uncorrelated errors (UN) and AR1 $(\rho=0.3)$. The results for uncorrelated errors are based on Eqs. (5.16) and (5.17) with contrast matrix $C=I_{3}$ and $C_{2}$ in costs per second. Costs were $C_{T}=€ 6000, C_{2}=€ 800 / \mathrm{h}$ $\approx € 13.33 / \mathrm{min}$ and $C_{1}=€ 150, € 400$ or $€ 600$. The number of stimulus types was $Q=3$, null block length was $14 \mathrm{~s}$, stimulus block length was $10 \mathrm{~s}$, TR and SOA were $2 \mathrm{~s}$. The horizontal lines indicate the minimum number of cycles of 2 . On the right $y$-axis for the optimal number of cycles the corresponding scanning time in minutes is given.

In Figure 5.3 the optimal number of cycles and in Figure 5.4 the optimal number of subjects is shown for varying autocorrelation $\rho$, different variance ratios $\sigma_{\epsilon}^{2} / \sigma_{q}^{2}$ and different cost ratios $C_{1} / C_{2}$. Figure 5.3 illustrates that for increasing autocorrelation the optimal number of cycles increases and the optimal number of subjects decreases except for the smallest variance ratio $\sigma_{\epsilon}^{2} / \sigma_{q}^{2}=1$ in combination with the lower cost ratios. This increasing and decreasing effect of the autocorrelation can also be seen in Figure 5.2 by comparing the results for uncorrelated error $(\rho=0)$ with correlated error $(\rho=0.3)$. Further just as in Figure 5.2, is it seen in Figures 5.3 and 5.4 that for a given autocorrelation and a given variance ratio the optimal number of cycles increases with increasing cost ratio whereas the optimal number of subjects decreases. Furthermore, the optimal number of cycles increases and the optimal number of subjects decreases in Figures 5.3 and 5.4 for increasing variance ratio. This generalizes the relationships in Figure 5.2 for $\rho=0$ and 
$\rho=0.3$ to other autocorrelation values.

An increase of optimal number of cycles for increasing autocorrelation was expected since for stronger correlated errors the different scans are stronger correlated and as a consequence each scan will provide less additional information than a scan in a model with lower autocorrelation. Therefore, more cycles are necessary for strongly correlated errors to obtain the same information as for weakly correlated errors. As a result of the increased optimal number of cycles, the optimal number of subjects decreases for increasing autocorrelation (see Figure 5.4 for an example).

In Table 5.2 the effect of highpass filtering on the optimal design is studied. A highpass filter was included into the matrix $S$ by a discrete cosine transform (DCT) set. We considered the effect of number of DCT basis functions from 1 to 5 basis functions for different autocorrelation coefficients $\rho$. The first basis function is a constant and corresponds to the vector $S=1_{N_{T}}$ which was used for the analytical derivations for uncorrelated errors. We considered here the same experimental parameters $(\mathrm{TR}=2 \mathrm{~s}, \mathrm{SOA}=2 \mathrm{~s}$, number of stimulus types $Q=3$, block length null equal to $14 \mathrm{~s}$ and stimulus block length equal to $10 \mathrm{~s}$ ) as before, total costs of $C_{T}=€ 6000$, costs per subject of $C_{1}=€ 200$ and costs per hour scanning time $C_{2}=€ 400 / \mathrm{h}$. The individual stimulus effects were of interest, thus $C=I_{3}$. Different variance ratios $\sigma_{\epsilon}^{2} / \sigma_{q}^{2}$ and different autocorrelations $\rho$ were considered. It can be seen in Table 5.2 that highpass filtering had in some cases an increasing effect on the number of cycles, i.e., the optimal number of cycles increased in some cases if the number of basis functions increased. As a consequence the optimal number of subjects decreased for an increasing number of basis functions. Further calculations showed that for a fixed number of basis functions and $\rho=0.3$, the optimal number of cycles increased and the optimal number of subjects decreased for increasing variance ratio. This is consistent with the previous results for uncorrelated errors and correlated errors without highpass filtering. The results for a highpass filter using Legendre polynomials were similar.

Table 5.2: Optimal number of cycles for different numbers of DCT basis functions, bf = basis function(s)

(a) Variance ratio $\sigma_{\epsilon}^{2} / \sigma_{q}^{2}=1$

\begin{tabular}{cccccc}
\hline & 1 bf & 2 bf & 3 bf & 4 bf & 5 bf \\
\hline$\rho=0$ & 2 & 2 & 2 & 2 & 3 \\
$\rho=0.2$ & 2 & 2 & 2 & 2 & 3 \\
$\rho=0.4$ & 2 & 2 & 2 & 3 & 3 \\
$\rho=0.6$ & 2 & 2 & 2 & 3 & 3 \\
\hline
\end{tabular}

(b) Variance ratio $\sigma_{\epsilon}^{2} / \sigma_{q}^{2}=10$

\begin{tabular}{cccccc}
\hline & 1 bf & 2 bf & 3 bf & 4 bf & 5 bf \\
\hline$\rho=0$ & 5 & 5 & 5 & 5 & 5 \\
$\rho=0.2$ & 6 & 6 & 6 & 6 & 6 \\
$\rho=0.4$ & 7 & 7 & 7 & 7 & 7 \\
$\rho=0.6$ & 8 & 8 & 8 & 8 & 8 \\
\hline
\end{tabular}




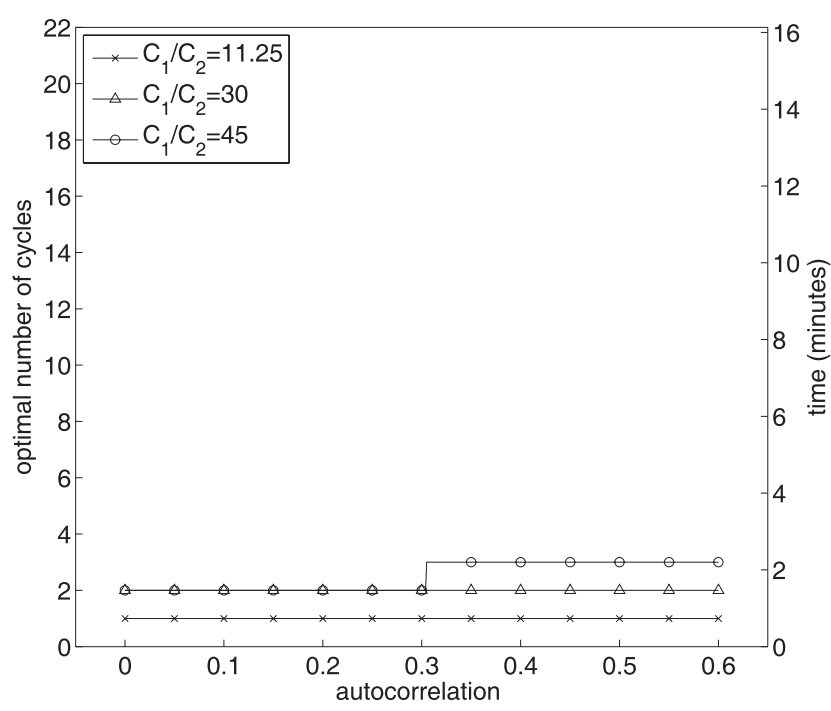

(a) Variance ratio $\sigma_{\epsilon}^{2} / \sigma_{q}^{2}=1$

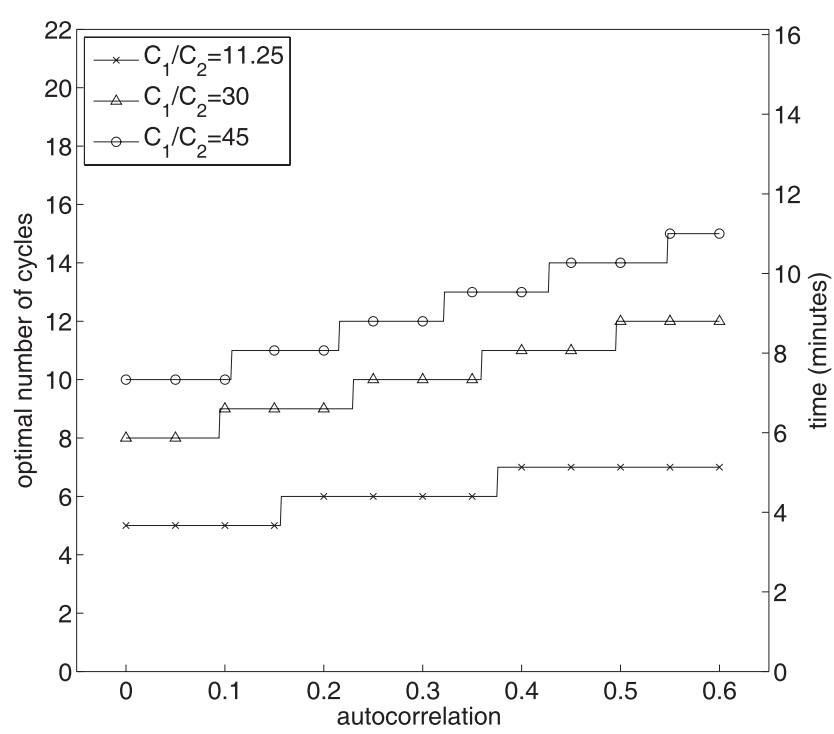

(c) Variance ratio $\sigma_{\epsilon}^{2} / \sigma_{q}^{2}=25$

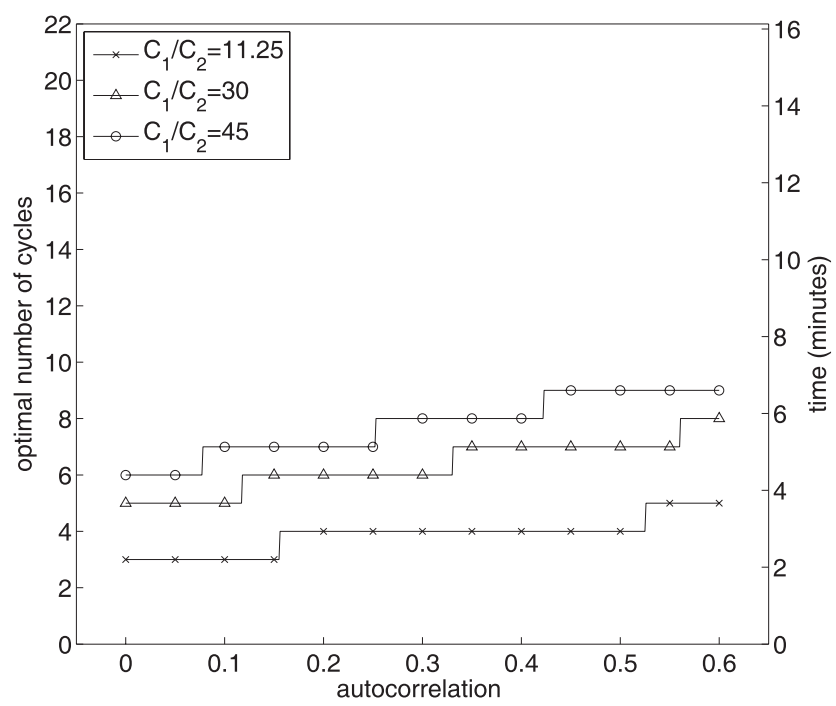

(b) Variance ratio $\sigma_{\epsilon}^{2} / \sigma_{q}^{2}=10$

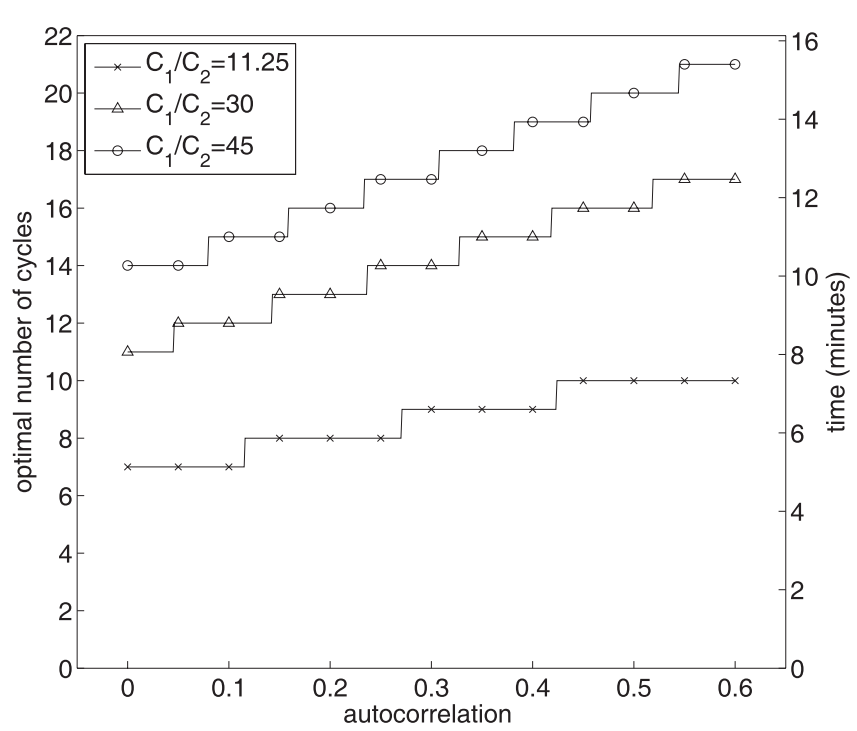

(d) Variance ratio $\sigma_{\epsilon}^{2} / \sigma_{q}^{2}=50$

Figure 5.3: Optimal number of cycles for different variance ratios $\sigma_{\epsilon}^{2} / \sigma_{q}^{2}$ depending on autocorrelation $\rho$. The following costs were assumed: $C_{T}=€ 6000, C_{2}=€ 800 / \mathrm{h} \approx$ $€ 13.33 / \mathrm{min}, C_{1}=€ 150, € 400$ or $€ 600$. The number of stimulus types was $Q=3$, contrast matrix $C$ was equal to $I_{3}$, null block length was $14 \mathrm{~s}$, stimulus block length was $10 \mathrm{~s}, \mathrm{TR}$ and SOA were $2 \mathrm{~s}$. 


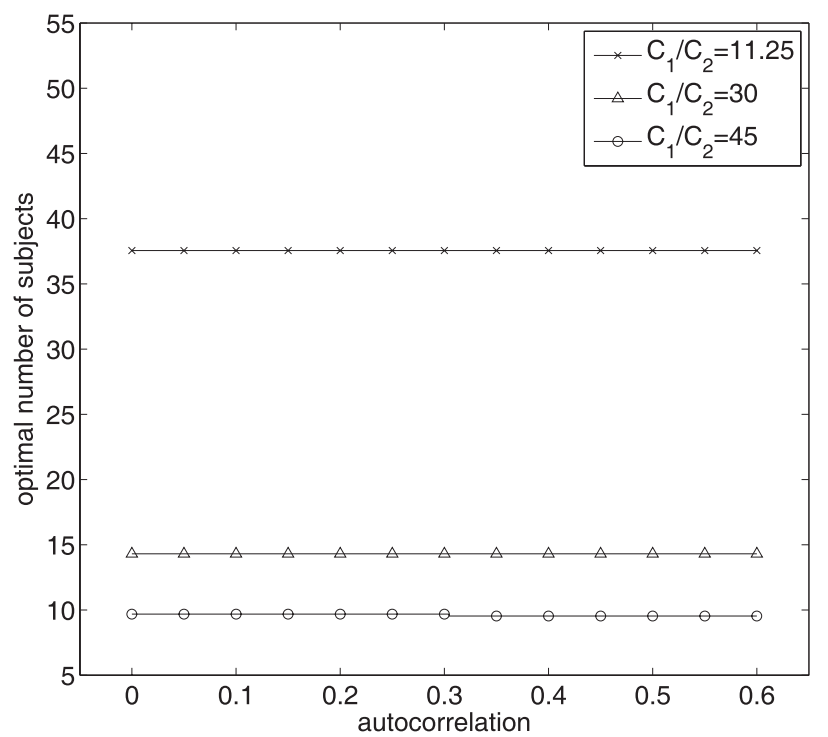

(a) Variance ratio $\sigma_{\epsilon}^{2} / \sigma_{q}^{2}=1$

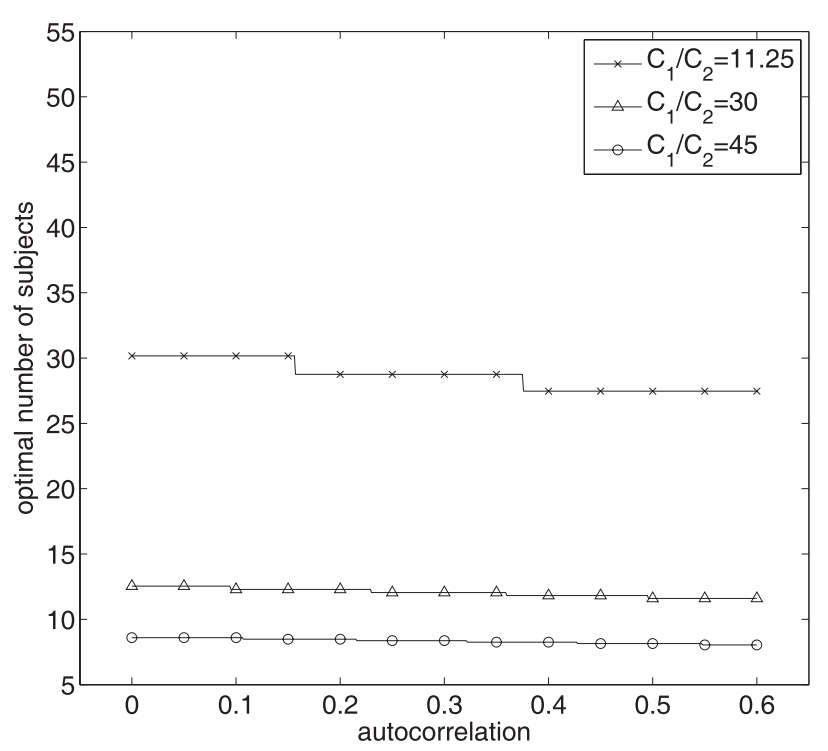

(c) Variance ratio $\sigma_{\epsilon}^{2} / \sigma_{q}^{2}=25$

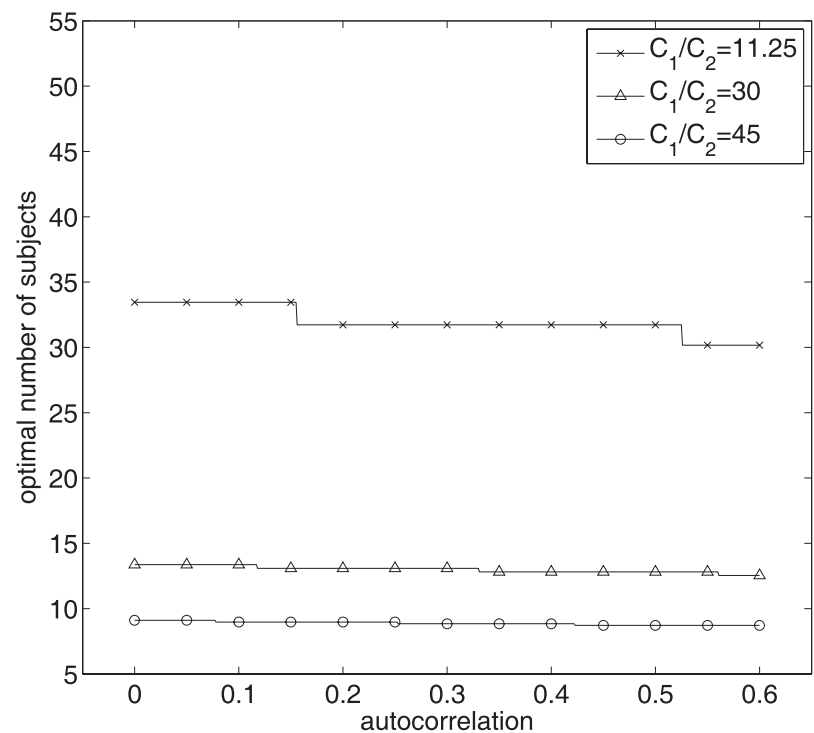

(b) Variance ratio $\sigma_{\epsilon}^{2} / \sigma_{q}^{2}=10$

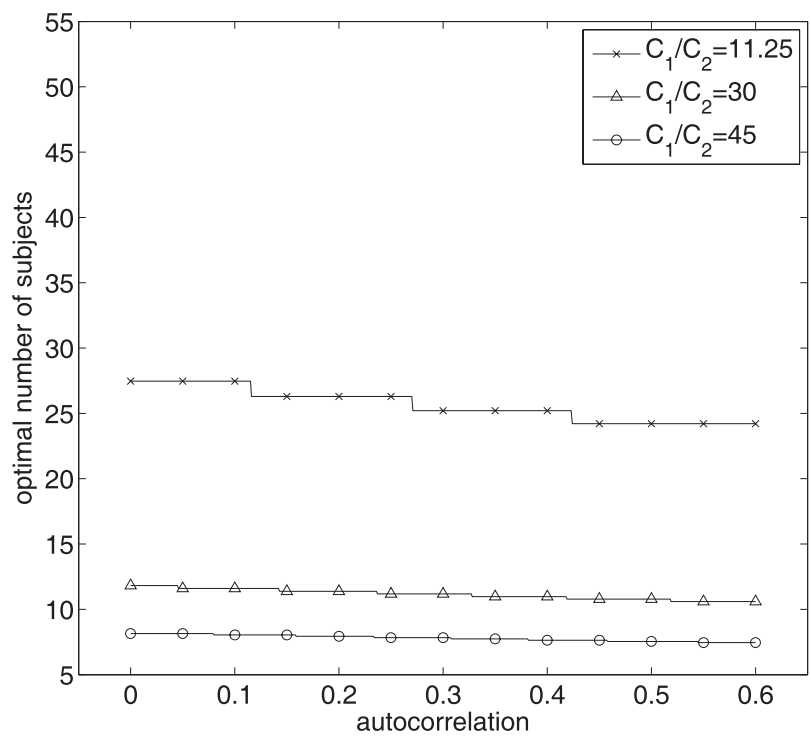

(d) Variance ratio $\sigma_{\epsilon}^{2} / \sigma_{q}^{2}=50$

Figure 5.4: Optimal number of subjects for different variance ratios $\sigma_{\epsilon}^{2} / \sigma_{q}^{2}$ depending on autocorrelation $\rho$. The following costs were assumed: $C_{T}=€ 6000, C_{2}=€ 800 / \mathrm{h} \approx$ $€ 13.33 / \mathrm{min}, C_{1}=€ 150, € 400$ or $€ 600$. The number of stimulus types was $Q=3$, contrast matrix $C$ was equal to $I_{3}$, null block length was $14 \mathrm{~s}$, stimulus block length was $10 \mathrm{~s}$, TR and SOA were $2 \mathrm{~s}$. 


\subsubsection{Effect of deviations from the optimal design for a given budget}

It can help researchers to plan their experiment and budget more efficiently if they have knowledge about the effect on design efficiency of deviations in the number of cycles and subjects from the optimal design. We will focus here on the effect of small deviations from the optimal design. The effect of deviations in the number of cycles and number of subjects from the optimal number of cycles and subjects can be evaluated by the relative efficiency. Assuming uncorrelated errors, it follows with Eqs. (5.12) and (5.13) that the relative efficiency of a design $\xi$ with $N_{C}$ cycles and $N$ subjects versus the optimal design $\xi^{*}$ with $N_{\text {Copt }}$ and $N_{\text {opt }}$ is given by:

$$
\operatorname{RE}\left(\xi \mid \xi^{*}\right)=\frac{N}{N_{o p t}} \cdot \frac{\frac{\sigma_{\epsilon}^{2}}{\sigma_{q}^{2}} \frac{1}{N_{C o p t}} \cdot \operatorname{trace}\left(C M^{-1} C^{T}\right)+\operatorname{trace}\left(C D C^{T}\right)}{\frac{\sigma_{\epsilon}^{2}}{\sigma_{q}^{2}} \frac{1}{N_{C}} \cdot \operatorname{trace}\left(C M^{-1} C^{T}\right)+\operatorname{trace}\left(C D C^{T}\right)}
$$

Keeping $N$ fixed and increasing or decreasing $N_{C}$ toward $N_{C o p t}$, the relative efficiency approaches $N / N_{\text {opt }}$. Furthermore, the relative efficiency increases for increasing $N_{C}$ but this increase attenuates for higher $N_{C}$ as $\left(\sigma_{\epsilon}^{2} / N_{C}\right) \operatorname{trace}\left(C M^{-1} C^{T}\right)$, the trace of the withinsubject part of the covariance matrix of $C \hat{\beta}_{G}$, decreases and $\operatorname{trace}\left(C D C^{T}\right)$, the trace of the between-subject part of the correlation matrix of $C \hat{\beta}_{G}$, then dominates the denominator of the second part in Eq. (5.21).

In Figure 5.5 the relative efficiencies for several designs versus the optimal design for estimation of individual effects $\left(C=I_{Q}\right)$ are given. The optimal number of cycles and subjects were calculated with the numerical program for correlated errors setting $\rho$ to 0 for uncorrelated errors and $\rho$ to 0.3 for AR1. The designs with small deviations in the number of cycles and subjects from the optimal design were chosen such that the number of cycles varied between $N_{C o p t} / 2$ and $N_{C o p t} \cdot 2$, and the number of subjects varied between $N_{\text {opt }}-3$ and $N_{\text {opt }}+3$ in Figure 5.5.

It can be seen in Figure 5.5 that several designs have a high relative efficiency, e.g., between 0.9 and 1.1. Thus, these designs with small deviations from the optimal design perform well in comparison to the optimal design but may not make full use of the budget of $€ 6000$ or exceed the budget. Figure 5.5 further illustrates that for a fixed number of subjects the relative efficiency attenuates if the number of cycles increases. This has consequences for designs with fewer subjects than the optimal design. For example in Figure $5.5 \mathrm{~b}$ for a variance ratio of 15 , the design with 23 subjects does not reach an efficiency very close to 1 , even when the number of cycles is twice the optimal value 6 . The attenuation was explained analytically for uncorrelated errors by Eq. (5.21). For correlated errors, it can also be seen in Figure 5.5 that the relative efficiency flattens off for higher numbers of cycles.

Another important comparison is between designs which employ the full budget $C_{T}$ and the optimal design for that budget. In Figure 5.6 we present the relative efficiencies versus the optimal design of designs with the same budget $C_{T}$ as the optimal design. It can be seen that several designs for both variance ratios and both error structures have a high relative efficiency above 0.9 in Figure 5.6. For a given value of $N_{C}$, the number of 


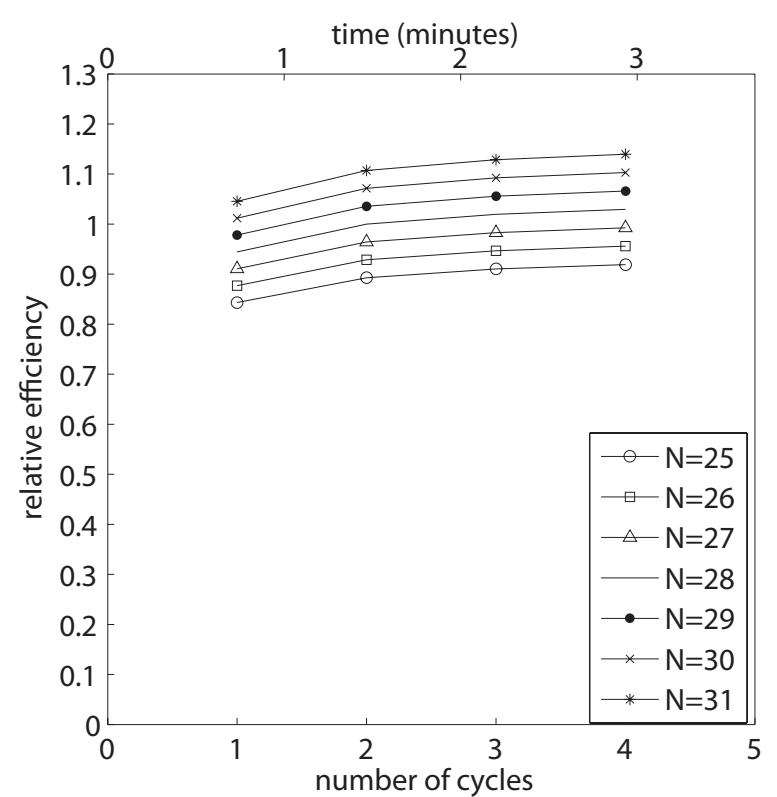

(a) Uncorrelated errors, $\sigma_{\epsilon}^{2} / \sigma_{q}^{2}=2, N_{\text {copt }}=$ $2, N_{\text {opt }}=28$

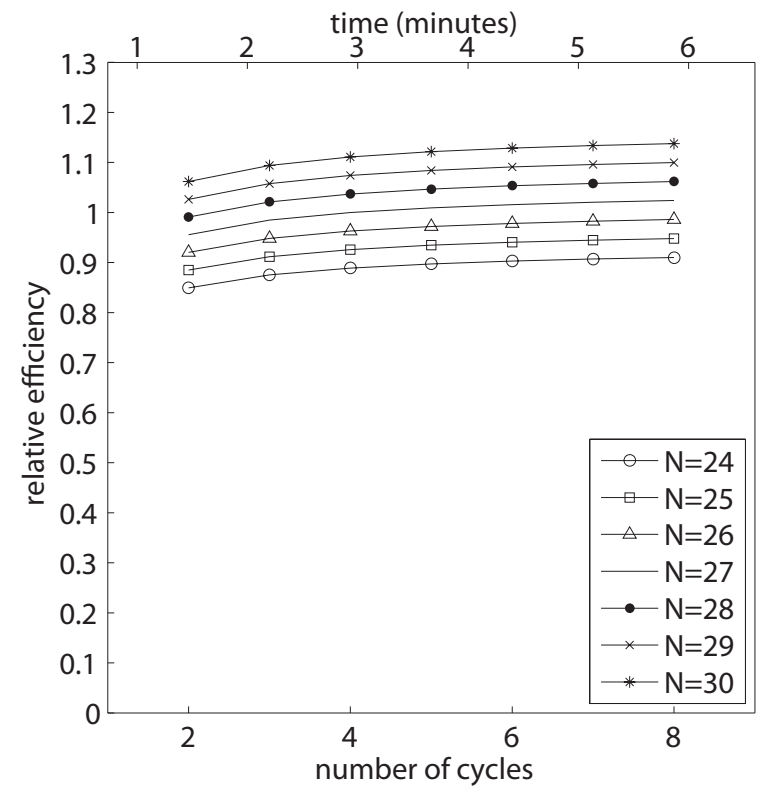

(c) $\operatorname{AR} 1(\rho=0.3), \sigma_{\epsilon}^{2} / \sigma_{q}^{2}=2, N_{\text {Copt }}=4$, $N_{\text {opt }}=27$

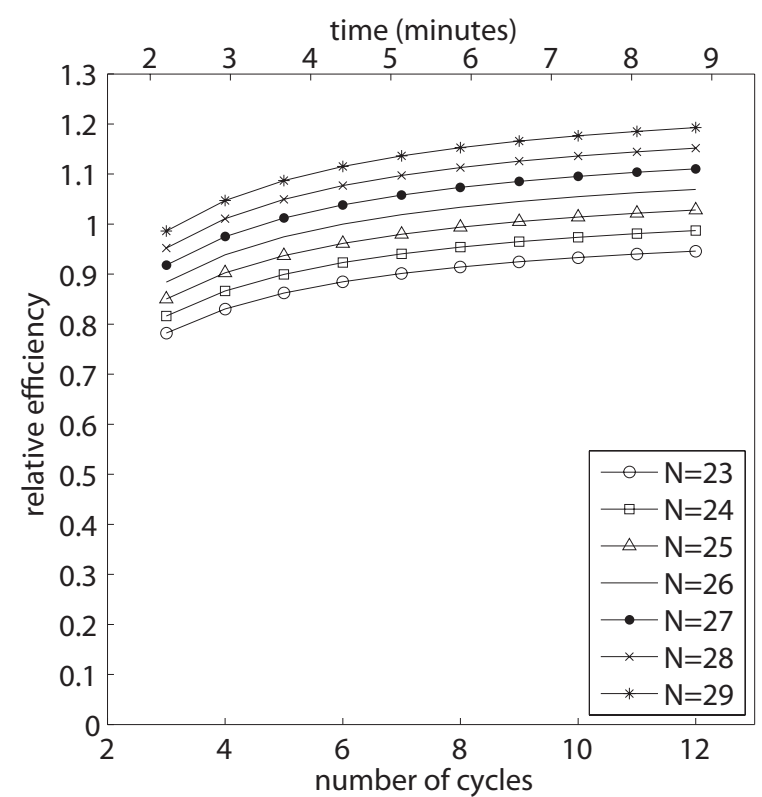

(b) Uncorrelated errors, $\sigma_{\epsilon}^{2} / \sigma_{q}^{2}=15, N_{C o p t}=6$, $N_{\text {opt }}=26$

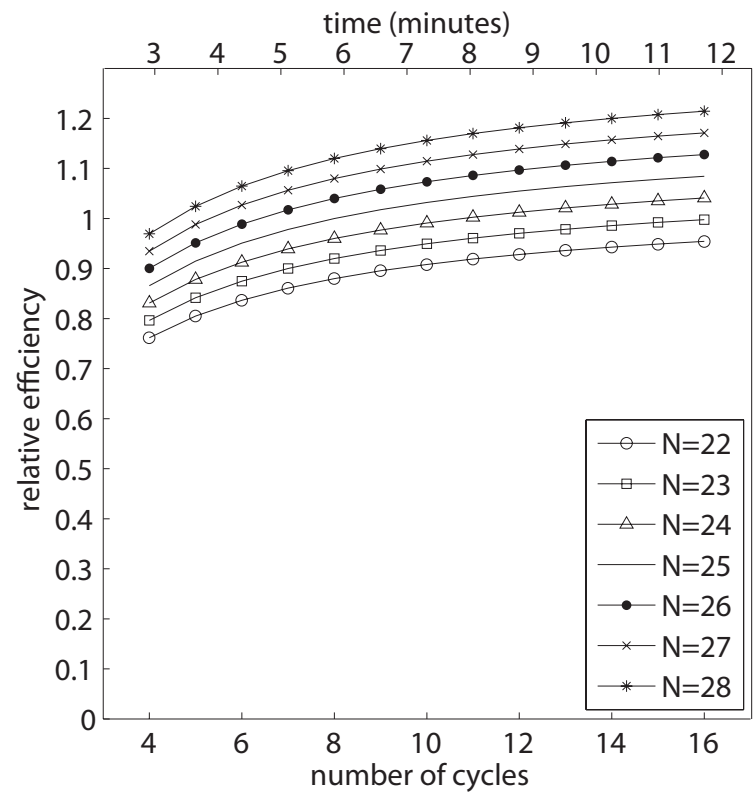

(d) $\operatorname{AR} 1(\rho=0.3), \sigma_{\epsilon}^{2} / \sigma_{q}^{2}=15, N_{\text {opt }}=8, N_{\text {opt }}=$ 25

Figure 5.5: Effect of small deviations from the optimal design for individual stimulus effects $\left(C=I_{3}\right)$. A TR of $2 \mathrm{~s}$ and the following design characteristics were assumed: $Q=3, \mathrm{SOA}=2 \mathrm{~s}, N_{B L_{0}}=7(14 \mathrm{~s}), N_{B L_{A}}=5(10 \mathrm{~s})$. The total costs $C_{T}$ were set to $€ 6000$. The costs per subject were $C_{1}=€ 200$ and the costs per scanning time were $C_{2}=$ $€ 400 / \mathrm{h} \approx 6.67 / \mathrm{min}$. 


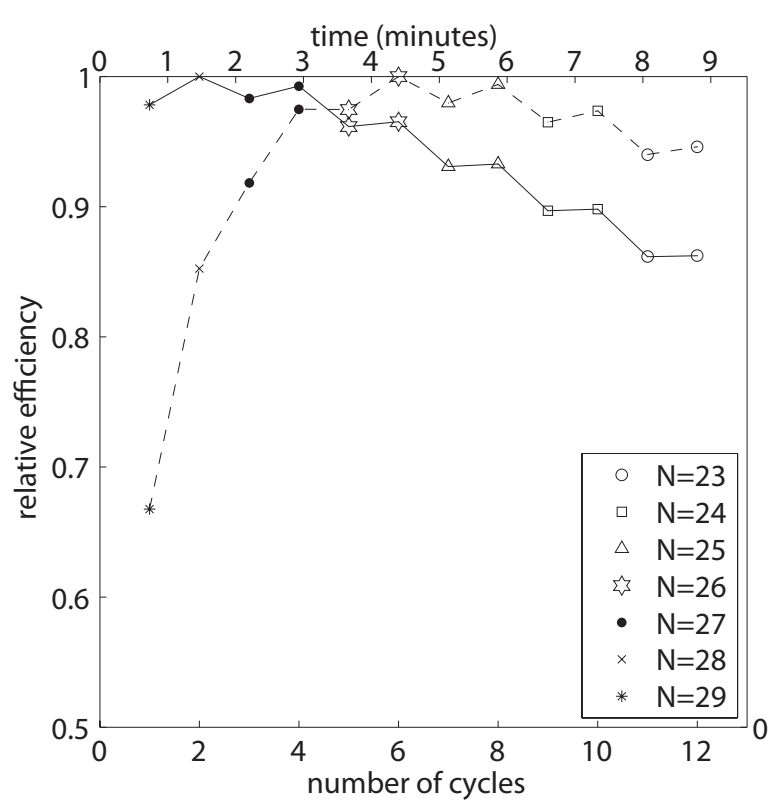

(a) Uncorrelated errors, $N_{\text {Copt }}=2$ and $N_{\text {opt }}=28$ $\left(\sigma_{\epsilon}^{2} / \sigma_{q}^{2}=2\right)$ or $N_{\text {Copt }}=6$ and $N_{\text {opt }}=26\left(\sigma_{\epsilon}^{2} / \sigma_{q}^{2}=\right.$ 15)

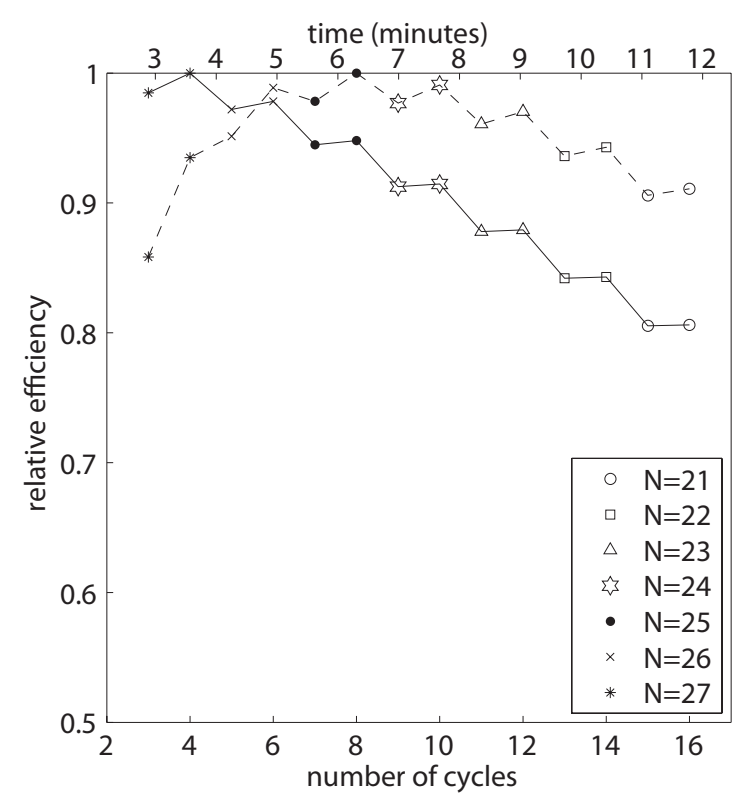

(b) AR1 $(\rho=0.3), N_{\text {Copt }}=4$ and $N_{\text {opt }}=27$ $\left(\sigma_{\epsilon}^{2} / \sigma_{q}^{2}=2\right)$ or $N_{\text {Copt }}=8$ and $N_{\text {opt }}=25$ $\left(\sigma_{\epsilon}^{2} / \sigma_{q}^{2}=15\right)$

Figure 5.6: Relative efficiency versus the optimal design of designs employing the full costs. The solid line is for variance ratio $\sigma_{\epsilon}^{2} / \sigma_{q}^{2}=2$ and the dashed line is for variance ratio $\sigma_{\epsilon}^{2} / \sigma_{q}^{2}=15$. The following assumptions were made: $Q=3, C=I_{3}$, TR $=2 \mathrm{~s}$, SOA $=2 \mathrm{~s}, N_{B L_{0}}=7(14 \mathrm{~s}), N_{B L_{A}}=5(10 \mathrm{~s}), C_{1}=€ 200, C_{2}=€ 400 / \mathrm{h} \approx € 6.66 / \mathrm{min}$.

subjects was calculated for costs of $C_{T}=€ 6000$ using the expression for $N$ in dependence of $N_{C}$ from Eq. (5.15) and rounded down to the nearest integer. In this way, the maximum possible number of subjects for a given number of cycles and costs was obtained and as a consequence, $\operatorname{trace}\left(\operatorname{Cov}\left(\hat{\beta}_{G}\right)\right)$ was minimized. As the number of cycles and the number of subjects were integer values, the full costs were often not completely employed. The costs varied in the range between $€ 5740$ and $€ 6000$ for both variance ratios. The number of cycles was chosen close to the optimal number of cycles for the different variance ratios of 2 and 15.

\subsubsection{Optimal design for a given power}

We will now explain how a given power can be obtained for a minimum budget. Firstly, the optimal number of cycles and subjects for a given budget have to be calculated. In our example a fixed budget $C_{T}=€ 4000$ is used together with the following parameters: $Q=2$, $\mathrm{TR}=\mathrm{SOA}=2 \mathrm{~s}$, null block length $14 \mathrm{~s}$, stimulus block length $10 \mathrm{~s}, C=\left[\begin{array}{ll}1 & -1\end{array}\right], C_{1}=$ $€ 200$ and $C_{2}=€ 400 / \mathrm{h} \approx 6.67 / \mathrm{min} \approx 0.11 / \mathrm{s}, \rho=0.3, \sigma_{\epsilon}^{2} / \sigma_{q}^{2}=2$ and between randomeffects correlation $D_{12}=0$. The interest is in the parameter contrast $\theta=\beta_{G 1}-\beta_{G 2}$. Using numerical computations as outlined in Section 5.2.4, the optimal number of cycles is then equal to 3 and the optimal number of subjects is equal to $18.93 \approx 19$.

Secondly, the power of this design with the derived optimal number of cycles and 
subjects is determined. If the power is lower or higher than the prespecified power, the variance $\operatorname{Var}(\hat{\theta})$, which guarantees the given level of power, has to be calculated. The power can be too high as we are interested in the minimum budget to obtain the prespecified power. The desired power in our example is $80 \%$ and the null-hypothesis is $H_{0}: \theta=0$ versus the alternative hypothesis $H_{A}: \theta=\Delta$. Specifying the effect size in percent signal change $\Delta=1 \%, \sigma_{\epsilon}^{2}=2, \sigma_{q}^{2}=1$ and a conservative significance level $\alpha=0.005$ to correct for testing at multiple voxels, a power of $63.48 \%$ is obtained which is too low. The following formula calculates the variance which gives a power of approximatively $(1-\delta)=0.80$ :

$$
\operatorname{Var}(\hat{\theta})=\left(\frac{\Delta}{z_{1-\delta}+z_{1-\alpha}}\right)^{2} .
$$

The $100(1-\alpha)$ percentile respectively the $100(1-\delta)$ percentile of the standard normal distribution is given by $z_{1-\alpha}$ respectively by $z_{1-\delta}$. In our example, $\operatorname{Var}(\hat{\theta})$ must be 0.0856 to obtain $80 \%$ power for effect size $\Delta=1 \%$.

Thirdly, the budget for this new variance has to be calculated. If the power is too low, the budget has to be raised and if the power is unnecessarily high, the budget has to be decreased. The new budget is given by

$$
C_{T}=\frac{C_{1}+C_{2} \cdot T_{S}}{\operatorname{Var}(\hat{\theta})} \cdot \sigma_{q}^{2}\left(\frac{\sigma_{\epsilon}^{2}}{\sigma_{q}^{2}} \operatorname{trace}\left(C\left(Z^{* T} Z^{*}\right)^{-1} C^{T}\right)+\operatorname{trace}\left(C D C^{T}\right)\right)
$$

This equation follows from Eqs. (5.11) and (5.15). The number of cycles is fixed in Eq. (5.23) to the previously calculated optimal value of 3 as Eq. (5.16) indicates that the optimal number of cycles does not depend on the budget $C_{T}$. Equation (5.23) results in a new budget of $€ 5130.74$. The optimal number of subjects can then be calculated by a new numerical computation which results in $N_{\text {opt }}=24.28 \approx 24$. This example shows how the optimal design with a minimum budget for a given power can be obtained.

\subsection{Discussion and conclusions}

The aim of this paper was to find the optimal design for multi-subject fMRI studies with respect to the scanning time and the number of subjects. We found the combination of number of subjects and number of cycles which was optimal according to the $A$-optimality criterion when both the number of subjects and the scanning time were restricted by experimental costs. Thus, our optimal design is the design which minimizes the trace of the group effects estimators' covariance matrix , respectively the trace of the contrasts estimators' covariance matrix, but simultaneously does not exceed experimental costs for a given cost function. Analytical solutions for the optimal number of cycles and optimal number of subjects were presented for blocked designs and uncorrelated errors. The analytical results are useful to describe the general relationships between the optimal number of subjects and cycles and the factors variance ratio, cost ratio and costs. These relationships were also valid for the numerical results for correlated errors with and without highpass filtering.

For correlated errors more cycles and fewer subjects are optimal than for uncorrelated errors. This can be explained by the decrease of information from individual scans when 
correlated errors are assumed instead of uncorrelated errors. The higher the correlation between scans is, the stronger the increase in optimal number of cycles and the stronger the decrease in optimal number of subjects from uncorrelated errors to correlated errors will be. The results for uncorrelated errors and correlated errors further show that for an increasing cost ratio, higher number of cycles become optimal. The explanation is that for higher cost ratios, cycles are becoming cheaper in relation to subjects and thus more cycles are optimal within the same total experimental costs.

The results for both error structures also illustrate that the optimal number of cycles increases and the optimal number of subjects decreases with increasing variance ratio. This can be explained by considering separately the three causes for an increasing variance ratio. Firstly, the variance ratio increases when $\sigma_{\epsilon}^{2}$ increases while $\sigma_{q}^{2}$ is fixed or increases less strongly than $\sigma_{\epsilon}^{2}$. In this first case a higher number of cycles is needed to estimate subject-specific effects efficiently from each subject which leaves less budget for subjects. Therefore, the optimal number of subjects decreases. Secondly, the variance ratio increases when $\sigma_{q}^{2}$ decreases while $\sigma_{\epsilon}^{2}$ is fixed or decreases less strongly than $\sigma_{q}^{2}$. In this second case a lower number of subjects becomes optimal so that more budget is available for cycles and the optimal number of cycles increases. Thirdly, the variance ratio increases when $\sigma_{\epsilon}^{2}$ increases and $\sigma_{q}^{2}$ decreases. As in the first case, more cycles are needed to estimate subject-specific effects efficiently and as in the second case, fewer subjects are needed so that more budget is available for cycles.

The effect of small deviations from the optimal design was studied. It was seen that small deviations from the optimal number of cycles and subjects had little effect on the relative efficiency. Keeping the number of subjects fixed and increasing the number of cycles, increases the relative efficiency, but this increase levels off with increasing number of cycles (see Figure 5.5). For uncorrelated errors, this behavior of the relative efficiency was explained by the analytical formula for the relative efficiency in Section 5.3.2. Such a point of diminishing return has also been found by Desmond \& Glover (2002) and Mumford $\&$ Nichols (2008) for power analyses. When the number of subjects and the number of cycles were chosen such that the total costs of $€ 6000$ were used, it was seen that several designs had high relative efficiencies. Similarly, Mumford \& Nichols (2008) found several designs with a power of $80 \%$ and costs of around $\$ 7600$.

Our results are based on minimizing the sum of the variances of the effects of interests. For one effect of interest, e.g., a single stimulus effect or a contrast between several stimulus effects, minimizing the variance of the estimator for this effect will lead to maximum power to test the null-hypothesis of zero effect or of a given effect value via a $t$-test. The value of this maximum power will of course depend on the effect size, the significance level $\alpha$, the within-subject and between-subject variance and might not reach a power of for example $80 \%$. However, instead of maximizing the power for a given budget our approach can also be used to find a design for a given power minimizing the budget. For multiple effects of interest, our method minimizes the sum of the variances of the estimators for the effects and the chosen optimal design is thus efficient for multiple univariate tests. Our method is thus not a replacement for power analyses but complementary to power analyses.

Mumford \& Nichols (2008) consider the power of $t$-tests for single hypotheses and of 
F-tests for simultaneous testing of multiple hypotheses. While our approach gives the optimal number of subjects and cycles for a given budget and maximum power of a $t$-test, it is not guaranteed that it will result in maximum power of an $F$-test. That can be seen as a limitation of our approach. By the cost function we are taking costs directly into account and find a specific optimal number of cycles and subjects minimizing the trace of the estimator's covariance matrix for a given budget. In contrast, Mumford \& Nichols (2008) first calculate several designs with $80 \%$ power and afterwards perform costs calculations for these designs. We are focusing on blocked design while Mumford \& Nichols (2008) consider event-related and blocked designs.

Desmond \& Glover (2002) also focused on blocked designs and calculated the power of $t$-tests for comparison of different conditions in a blocked design. In contrast to our work and Mumford \& Nichols (2008), Desmond \& Glover (2002) did not consider correlated errors for their power calculations and no convolution with a hemodynamic response function. However, Mumford \& Nichols (2008) showed that power calculations are incorrect if temporal correlation and HRF convolution are ignored. Likewise, our results illustrate that the optimal number of subjects and cycles can differ between correlated and uncorrelated errors. Desmond \& Glover (2002) provided a more time-intensive simulation based method whereas our approach results in direct analytical formulae for uncorrelated errors and typically less time-intensive numerical computations for correlated errors.

To apply our results, it is important to remember that the cost ratio depends on the unit in time. In Eqs. (5.16) and (5.17) the costs $C_{2}$ are given per second scanning time. Furthermore, the random-effects variance $\sigma_{q}^{2}$ depends on the scaling of the HRF. For example, if the amplitude of the HRF is $m$ at its peak and the HRF is scaled by multiplication with $1 / m$ to obtain an amplitude of 1 , the random-effects variance will become $m^{2}$ as large as every random effect $b_{i}$ will become $m$ times as large. The variance ratio of within- to between-subject variance has to be determined from previous studies. It would further be interesting to find a design which is efficient for a possible range of parameter values, e.g., for a possible range of the variance ratio $\sigma_{\epsilon}^{2} / \sigma_{q}^{2}$. For example, a maximin design (Atkinson et al., 2007), which is robust against misspecification of $\sigma_{\epsilon}^{2} / \sigma_{q}^{2}$ in a prespecified range, could be determined. In the following we will present more possible extensions of our work and discuss the assumptions which were made.

It was assumed that the error covariance matrix is the same for all subjects. While this is a simplification, it is a common assumption in optimal design as a parsimonious model is needed as a basis for further optimal design calculations. When the error covariance matrix $\sigma_{\epsilon}^{2} \Sigma$ is estimated from a previous study, heterogeneity can be taken into account. A different error covariance matrix can be estimated per subject and subsequently an average error covariance matrix can be determined for use in our equations. For example for an AR1 structure, the average correlation parameter and average error variance can be used for further calculations. The assumption of equal random-effects variance for all stimulus effects is also useful to have a parsimonious model as basis for optimal design calculation. The analytical results for uncorrelated errors were based on the following assumptions: $\mathrm{TR}=\mathrm{SOA}$, only filtering of constant trend $\left(S=1_{N_{T}}\right)$ and inshifting. The assumption of inshifting is explained in Appendix 5.A. Further, it is discussed in Appendix 5.A why this 
assumption has little to no effect on the results. If the other assumptions do not hold, the numerical program can be used to determine the optimal number of cycles and subjects as no assumption on the TR and SOA or the degree of highpass filtering are necessary for the MATLAB code.

Another often used optimality criterion is the $D$-optimality criterion which minimizes the determinant of the covariance matrix of the estimators for the unknown parameters (Atkinson et al., 2007). One advantage of the $D$-optimality criterion is that the $D$-optimal design is invariant to linear transformation of the regressors in the model. However, the regressors for the stimulus effects $\beta_{G i}$ all depend on the scaling of the hemodynamic response function and the $A$-optimal design is, like the $D$-optimal design, invariant to scaling of the HRF. Future work could include the application of the $D$-optimality criterion. For this extension the off-diagonal elements of the random-effects correlation matrix $D$ in Eq. (5.9) have to be considered not only for contrasts but also for individual stimulus effects. For the $A$-optimality criterion, consideration of the off-diagonal elements is only necessary for contrasts. Furthermore, an extension to multiple runs per session is important. Our current results consider experiments with one run and one session. Increasing the number of runs will have a decreasing effect on the trace of the covariance matrix of the estimators but likely this effect will attenuate for higher number of runs similar to the effect of the number of cycles on the relative efficiency.

Another extension is the consideration of across-subject contrasts. For example, Suckling et al. (2008) studied the power for comparison of two groups in a two-center repeatedmeasures pharmacological fMRI study and Schouten (1999) presents the optimal allocation ratio of subjects into two groups depending on the costs in a clinical trial. The difference in means between two independent groups can be considered via an unpaired $t$-test. The variance of such a between-subject contrast is a pooled sum of the variances of the groupspecific estimates. Assuming equal subjects costs, scanning time costs, error variance, autocorrelation and random-effects variances for both groups, the costs per group will be half of the total costs and equal sample sizes, i.e., equal number of subjects and equal scanning time, per group are optimal. The variances of the group-specific estimates can then be minimized by our approach using $C_{T} / 2$ per group and the optimal number of cycles and subjects per group can be determined. By minimizing the variances of the group-specific estimates, we minimize the variance of the between-subject contrast. Unequal costs, autocorrelation or variances between groups affect the optimal budget split between groups, but given a budget split, optimization can be performed per group to optimize between-group estimation.

Summarizing, we presented a method to determine the optimal number of cycles and subjects for a blocked multi-subject fMRI experiment. This method takes design efficiency and costs into consideration. Therefore, efficiency can be gained and costs can be reduced by using this method to determine the optimal number of cycles and optimal number of subjects. Typically, fMRI noise is temporally correlated and highpass filtering is applied. Therefore, we recommend to use numerical computations for determination of the optimal number of subjects and cycles. The analytical formulae are useful to describe the general relationships between the optimal number of subjects and cycles and other parameters. 


\section{A Expression for $Z^{* T} Z^{*}$ in Section 5.2.2 assuming uncorrelated errors}

\section{A.1 General expression}

We will show that the matrix $Z^{* T} Z^{*}$ can be expressed as $N_{C} \cdot M$, where $N_{C}$ is the number of cycles of $A_{1} \ldots A_{Q} N$ and $M / \sigma_{\epsilon}^{2}$ is the $Q \times Q$ information matrix of one cycle $A_{1} \ldots A_{Q} N$. The matrix $Z^{* T} Z^{*}$ is given by

$$
Z^{* T} Z^{*}=Z^{T}\left(I-P_{V S}\right) Z
$$

For uncorrelated errors, it holds that $P_{V S}=P_{S}$ and $V=I_{N_{T}}$. Furthermore, we assume that $S=(1, \ldots, 1)^{T}$. The projection matrix $P_{S}$ projects onto the space spanned by the vector $S$. The matrix $P_{S}$ equals $\left(1 / N_{T}\right) \cdot J_{N_{T} \times N_{T}}$ with $J_{N_{T} \times N_{T}}$ being the $N_{T} \times N_{T}$ matrix with all elements equal to 1 . Thus, $I-P_{S}$ is a centering matrix here, and $Z^{*}=\left(I-P_{S}\right) Z$ is the centered design matrix.

The design matrix $Z=X\left(I_{Q} \otimes h\right)$ is obtained by multiplication of the matrix $X$ with the Kronecker product $I_{Q} \otimes h$. The matrix $X$ contains 0's and 1's indicating a stimulus type at a certain time point and will be explained in detail below. The vector $h=\left(h_{1}, \ldots, h_{N_{h}}\right)^{T}$ is the hemodynamic response function sampled at $N_{h}$ time points with sampling rate TR. To derive Eq. (5.29) and as a consequence the analytical results for uncorrelated errors in Section 5.3.1.1, the time between trials in one block, the stimulus onset asynchrony (SOA), was assumed to be equal to TR. Therefore, the hemodynamic response function is here sampled with rate TR. The hemodynamic response function, e.g., the canonical double gamma function (Friston et al., 1998; Henson, 2004), is assumed to be known.

The $N_{T} \times\left(Q N_{h}\right)$ dimensional matrix $X$ is a block matrix containing the stimulus convolution matrices $X_{q}\left(\right.$ Dale, 1999) for the different stimulus types $A_{q}: X=\left(X_{1} \ldots X_{Q}\right)$. Each $N_{T} \times N_{h}$ matrix $X_{q}$ consists of 0 and 1. The first column of $X_{q}$ is the actual stimulus sequence $s^{q}$ where 1 indicates an occurrence of stimulus $A_{q}$ and 0 indicates no occurrence of stimulus $A_{q}$. The other columns of $X_{q}$ are shifted versions of the stimulus sequence to model convolution of the stimulus sequence $s^{q}$ with the hemodynamic response function. When the stimulus sequence is shifted, zeros are filled in:

$$
X_{q}=\left(\begin{array}{cccc}
s_{1}^{q} & 0 & \ldots & 0 \\
s_{2}^{q} & s_{1}^{q} & \ddots & \vdots \\
\vdots & \vdots & \ddots & 0 \\
s_{N_{T}}^{q} & s_{\left(N_{T}-1\right)}^{q} & \ldots & s_{\left(N_{T}-N_{h}+1\right)}^{q}
\end{array}\right)
$$

We call this zero padding. However, for our following derivations we will assume that the 
outshifted elements in the columns of the matrices $X_{q}$ are shifted in again:

$$
X_{q}=\left(\begin{array}{cccc}
s_{1}^{q} & s_{N_{T}}^{q} & \ldots & s_{\left(N_{T}-N_{h}+2\right)}^{q} \\
s_{2}^{q} & s_{1}^{q} & \ddots & s_{\left(N_{T}-N_{h}+3\right)}^{q} \\
\vdots & \vdots & \ldots & \vdots \\
s_{N_{T}}^{q} & s_{\left(N_{T}-1\right)}^{q} & \ldots & s_{\left(N_{T}-N_{h}+1\right)}^{q}
\end{array}\right) .
$$

This will be called "inshifting". The assumption of inshifting is a theoretical assumption which is necessary to derive the optimal number of cycles for uncorrelated errors. Due to the assumption of inshifting, the matrices $M$ and $Z^{* T} Z^{*}$ are only invertible if null blocks are included in the design. We define the centered matrix $X^{*}$ by $X^{*}=\left(I-P_{S}\right) X$. The assumption of inshifting had little to no effect on the results when we compared results under the assumption of inshifting to results under the assumption of zero padding. As the null block is assumed at the end of a cycle $A_{1} \ldots A_{Q} N$, both assumptions often lead to the same design matrices. Furthermore, the more scans are obtained, the less the matrix $X^{* T} X^{*}$ under the assumption of inshifting will differ from $X^{* T} X^{*}$ under the assumption of zero padding.

It follows with the definition of $X^{*}=\left(I-P_{S}\right) X$ that

$$
Z^{* T} Z^{*}=\left(I_{Q} \otimes h\right)^{T} X^{* T} X^{*}\left(I_{Q} \otimes h\right) .
$$

For all stimulus types equal stimulus frequency $p$ is assumed. This means that each stimulus occurs equally often in the experiment and that all blocks for all different stimulus types have the same block length. We denote by $N_{A}$ the number of times that stimulus $A_{q}$ occurs in the design for one subject. So, $p=N_{A} / N_{T}$ and $X^{*}=X-p 1_{N_{T} \times\left(Q N_{h}\right)}$. The matrix $X^{* T} X^{*}$ consists of the sub-matrices $X_{q_{1}}^{* T} X_{q_{2}}^{*}$ :

$$
X^{* T} X^{*}=\left(\begin{array}{cccc}
X_{1}^{* T} X_{1}^{*} & X_{1}^{* T} X_{2}^{*} & \ldots & X_{1}^{* T} X_{Q}^{*} \\
X_{2}^{* T} X_{1}^{*} & X_{2}^{* T} X_{2}^{*} & & \vdots \\
\vdots & & \ddots & \vdots \\
X_{Q}^{* T} X_{1}^{*} & \ldots & \ldots & X_{Q}^{* T} X_{Q}^{*}
\end{array}\right)
$$

In Appendix 5.B it is shown that the entry $\left(X_{q_{1}}^{* T} X_{q_{2}}^{*}\right)_{m n}$ can be expressed as

$$
\left(X_{q_{1}}^{* T} X_{q_{2}}^{*}\right)_{m n}=N_{C} \cdot(1-p)^{2} \cdot P_{1}+N_{C} \cdot(-p)^{2} \cdot P_{2}+N_{C} \cdot(1-p)(-p) \cdot P_{3} .
$$

The factors $P_{1}, P_{2}$ and $P_{3}$ give the number of products of form $(1-p)^{2},(-p)^{2}$ and $(-p)(1-$ $p)$. The number of these products depends further on the shift size $d$ which is the shift between column $m$ in $X_{q_{1}}^{*}$ and column $n$ in $X_{q_{2}}^{*}$ :

$$
\begin{aligned}
& P_{1}=\max \left(N_{B L_{A}}-d, 0\right)+\max \left(0, d-N_{B L_{0 T}}\right) \\
& P_{2}=\max \left(N_{B L_{0 T}}-d, 0\right)+\max \left(0, d-N_{B L_{A}}\right) \\
& P_{3}=\min \left(d, N_{B L_{T}}-d, N_{B L_{O T}}\right) .
\end{aligned}
$$

The shift size $d$ is given as $|(m-n)+| q_{1}-q_{2}\left|N_{B L_{A}}\right|$. The total length of one cycle $A_{1} \ldots A_{Q} N$ is $N_{B L_{T}}=Q N_{B L_{A}}+N_{B L_{0}}$ and the total length of one null block in sequence $s^{q}$ is given by $N_{B L_{0 T}}=(Q-1) N_{B L_{A}}+N_{B L_{0}}$. It follows that

$$
X^{* T} X^{*}=N_{C} K,
$$


where $K$ is a $\left(Q N_{h}\right) \times\left(Q N_{h}\right)$ dimensional matrix dependent on $N_{B L_{0}}$ and $N_{B L_{A}}$ but independent of $N_{C}$. We define

$$
M=\left(I_{Q} \otimes h\right)^{T} K\left(I_{Q} \otimes h\right) .
$$

The matrix $M$ depends on the block lengths $N_{B L_{0}}, N_{B L_{A}}$ and the vector $h$ but is independent of $N_{C}$. The vector $h$ itself depends on the chosen hemodynamic response function, the number of sampling points $N_{h}$ for the HRF and the sampling rate for the HRF which is assumed to be equal to TR. It follows that

$$
Z^{* T} Z^{*}=N_{C} M
$$

\section{A.2 Approximated expression for matrix $M$ in Section 5.3.1.1}

In this section, we give the following approximation for the $Q \times Q$ matrix $M$ in Eq. (5.34):

$$
M=C_{Q} \cdot N_{B L_{0}} \cdot f(p, Q) \cdot H,
$$

where we denote by $H$ the sum $\sum_{m=1}^{N_{h}} \sum_{n=1}^{N_{h}} h_{m} h_{n}$ and $f(p, Q)=p(1-p) /(1-Q p)$ which is defined for a stimulus frequency $p$ in the interval $[0,1 / Q)$. This approximation for the information matrix $M$ can be seen as an extension to the approximation for the information matrix $X^{* T} X^{*}$ in Liu \& Frank (2004). The $Q \times Q$ matrix $C_{Q}$ is given by

$$
C_{Q}=\left(1+\frac{p}{1-p}\right) I_{Q}-\frac{p}{1-p} 1_{Q} 1_{Q}^{T}
$$

The matrix $C_{Q}$ is of format $Q \times Q$ and gives the correlation between the detrended stimulus sequences $s^{q *}$. Thus, the diagonals of $C_{Q}$ contain 1 and the off-diagonal elements of $C_{Q}$ are equal to $-p /(1-p)$.

We will derive this approximation by assuming $N_{h}=1$ and $h=1$ (first simplification) or $Q=1$ and $N_{B L_{0}}=N_{B L_{A}}$ (second simplification). Let us assume that $N_{h}=1$ and $h=1$. Then

$$
\begin{aligned}
\left(Z^{* T} Z^{*}\right)_{q q} & =h^{T}\left(X_{q}^{* T} X_{q}^{*}\right) h \\
& =N_{C} \cdot\left((1-p)^{2} N_{B L_{A}}+(Q-1)(-p)^{2} N_{B L_{A}}+(-p)^{2} N_{B L_{0}}\right) .
\end{aligned}
$$

It follows from the definition of the stimulus frequency $p=N_{B L_{A}} /\left(Q N_{B L_{A}}+N_{B L_{0}}\right)$ that $N_{B L_{A}}=p /(1-Q p) N_{B L_{0}}$. We define $g(p, Q)=p /(1-Q p)$ and it follows that

$$
\begin{aligned}
\left(Z^{* T} Z^{*}\right)_{q q} & =N_{C} \cdot\left((1-p)^{2} g(p, Q) N_{B L_{0}}+(Q-1)(-p)^{2} g(p, Q) N_{B L_{0}}+(-p)^{2} N_{B L_{0}}\right) \\
& =N_{C} \cdot N_{B L_{0}} \underbrace{\left((1-p)^{2} g(p, Q)+(Q-1)(-p)^{2} g(p, Q)+(-p)^{2}\right)}_{=: f(p, Q)} .
\end{aligned}
$$

We calculate that $f(p, Q)=p(1-p) /(1-p Q)$. Furthermore, it can be calculated that

$$
\begin{aligned}
\left(Z^{* T} Z^{*}\right)_{q_{1} q_{2}} & =h^{T}\left(X_{q_{1}}^{* T} X_{q_{2}}^{*}\right) h \\
& =N_{C} \cdot\left((1-p)(-p) N_{B L_{A}}+(1-p)(-p) N_{B L_{A}}+p^{2}(Q-2) N_{B L_{A}}+p^{2} N_{B L_{0}}\right) \\
& =N_{C} \cdot N_{B L_{0}} \cdot\left(-2 p(1-p) g(p, Q)+p^{2}(Q-2) g(p, Q)+p^{2}\right) \\
& =N_{C} \cdot N_{B L_{0}} \cdot\left(\frac{-p^{2}}{1-p Q}\right) \\
& =N_{C} \cdot N_{B L_{0}} f(p, Q) C_{Q_{q_{1} q_{2}}} .
\end{aligned}
$$


This shows Eq. (5.36) for the first simplification $\left(N_{h}=1\right.$ and $\left.h=1\right)$.

For the second simplification, we assume that $Q=1$ and $N_{B L_{A}}=N_{B L_{0}}$ so that $Z^{* T} Z^{*}$ is a scalar and $p=1 / 2$. By $h_{i}$ the $i$ th element in the vector $h$ is denoted. It holds that

$$
\begin{aligned}
\left(Z^{* T} Z^{*}\right) & =h^{T} X^{* T} X^{*} h \\
& =\sum_{n=1}^{N_{h}} \sum_{m=1}^{N_{h}} h_{m}\left(X^{* T} X^{*}\right)_{m n} h_{n} .
\end{aligned}
$$

We know that

$$
\begin{aligned}
& \left(X^{* T} X^{*}\right)_{m n} \\
= & N_{C}\left[(1-p)^{2} \mid N_{B L}-\text { shift size }\left|+(-p)^{2}\right| N_{B L}-\text { shift size } \mid+2(-p)(1-p) \text { shift size }\right] \\
= & N_{C} \cdot N_{B L} \cdot \\
& {\left[(1-p)^{2} \frac{\mid N_{B L}-\text { shift size } \mid}{N_{B L}}+(-p)^{2} \frac{\mid N_{B L}-\text { shift size } \mid}{N_{B L}}+2(-p)(1-p) \frac{\text { shift size }}{N_{B L}}\right] . }
\end{aligned}
$$

We make the following approximations which will be more precise for small shift sizes and/or a larger number of trials within a block

$$
\begin{aligned}
\frac{\mid N_{B L}-\text { shift size } \mid}{N_{B L}} & \approx 1 \\
\frac{\text { shift size }}{N_{B L}} & \approx 0 .
\end{aligned}
$$

We obtain then

$$
\begin{aligned}
\left(Z^{* T} Z^{*}\right) & =\sum_{n=1}^{N_{h}} \sum_{m=1}^{N_{h}} h_{m}^{T}\left(X^{* T} X^{*}\right)_{m n} h_{n} \\
& =N_{C} \cdot N_{B L} \cdot \frac{1}{2} \sum_{m=1}^{N_{h}} \sum_{n=1}^{N_{h}} h_{m} h_{n} .
\end{aligned}
$$

Note that for $p=1 / 2$ and $Q=1$ it holds that $f(p, Q)=1 / 2$. This shows Eq. (5.36) for the second simplification.

By the derivations above we motivated our approximation in Eq. (5.36) based on two simplifications. We calculate now the inverse of $M$ from Eq. (5.36). The inverse of $M$ is given by

$$
M^{-1}=\frac{1}{N_{B L_{0}} f(p, Q) H} C_{Q}^{-1}
$$

Using Woodbury's formula (Harville, 1997, p.424) it follows that for $p<1 / Q$

$$
C_{Q}^{-1}=(1-p) I_{Q}+\frac{p(1-p)}{1-Q p} 1_{Q} 1_{Q}^{T}
$$


Finally, the following expression, which will be useful for simplification of Eqs. (5.16) and (5.17), is obtained

$$
\begin{aligned}
\operatorname{trace}\left(M^{-1}\right) & =\frac{1}{N_{B L_{0}} f(p, Q) H} \operatorname{trace}\left(C_{Q}^{-1}\right) \\
& =\frac{1}{N_{B L_{0}} f(p, Q) H}\left(Q(1-p)+\frac{Q p(1-p)}{1-Q p}\right) \\
& =\frac{Q-Q^{2} p+Q p}{p N_{B L_{0}} H} .
\end{aligned}
$$

We tested this form of the approximation for various combinations of $p(p=1 /(Q+\sqrt{Q})$, the $A$-optimal stimulus frequency for estimation of individual stimulus effects, or $p=$ $1 /(Q+1))$, TR respectively SOA $(1 \mathrm{~s}, 2 \mathrm{~s}), N_{h}(1,17,33), Q(1,2,3)$ and block length of null blocks $(10 \mathrm{~s}, 11 \mathrm{~s}, \ldots, 30 \mathrm{~s})$. For the $A$-optimal frequency, the block length of stimulus blocks was given by SOA $\cdot\left[(1 / \sqrt{Q}) \cdot\left(B L_{0} / \mathrm{SOA}\right)\right]$, where $[x]$ denotes the nearest integer number to the real number $x$ and $B L_{0}$ is the length of null blocks in seconds. For most combinations the approximation was accurate, i.e., the trace of the real matrix $M^{-1}$ was $92 \%$ to $107 \%$ of the trace of the approximated matrix $M^{-1}$. For smaller block lengths the approximation was less accurate. In particular, for stimulus frequency $p=1 /(Q+1)$ the given range was $92 \%$ to $104 \%$ for all block lengths higher than or equal to $12 \mathrm{~s}$. For $p=1 /(Q+\sqrt{Q})$, the range for $Q=2$ was $93 \%$ to $106 \%$ only for block lengths higher than or equal to $11 \mathrm{~s}$ and the range for $Q=3$ was $94 \%$ to $107 \%$ only for block lengths higher than or equal to $16 \mathrm{~s}$. Note that for $Q=1$ the stimulus frequencies $p=1 /(Q+\sqrt{Q})$ and $p=1 /(Q+1)$ are equal and the range is as given above for $p=1 /(Q+1)$.

\section{B Calculation of $X^{* T} X^{*}$ in Eq. (5.29)}

The product $X^{* T} X^{*}$ in Eq. (5.29) will be calculated by making firstly two simplifications in Appendix 5.B.1, i.e., the assumption of one stimulus type and the assumption of stimulus block length being equal to null block length. Based on the results in Appendix 5.B.1, the product $X^{* T} X^{*}$ can be calculated generally for one stimulus type in Appendix 5.B.2 without the assumption of stimulus block length being equal to null block length. Finally in Appendix 5.B.3, $X^{* T} X^{*}$ for more than one stimulus type without any assumption on block lengths is given.

\section{B.1 Calculation for one stimulus type and stimulus block length equal to null block length}

We consider blocked designs with equal number of trials $N_{B L}$ per block for null events and stimulus events and with block order $A N$, i.e., an $A$ block is always followed by a null block $N$ so that the design has form $A N A N \ldots A N$. We will speak of one cycle $A N$, when we refer to an $A$ block and a null block $N$ together. It will be shown that for all $m=1, \ldots, N_{h}$ 
and for all $n=1, \ldots, N_{h}$ :

$$
\begin{aligned}
& \left(X^{* T} X^{*}\right)_{m n} \\
= & N_{C}\left[(1-p)^{2} \cdot\left|N_{B L}-\bmod \left(|m-n|, 2 N_{B L}\right)\right|\right. \\
& +(-p)^{2} \cdot\left|N_{B L}-\bmod \left(|m-n|, 2 N_{B L}\right)\right| \\
& \left.+2(-p)(1-p) \cdot \min \left(\bmod \left(|m-n|, 2 N_{B L}\right), 2 N_{B L}-\bmod \left(|m-n|, 2 N_{B L}\right)\right)\right] \\
=: & N_{C} K_{m n} .
\end{aligned}
$$

The entry $\left(X^{* T} X^{*}\right)_{m n}$ of the matrix $X^{* T} X^{*}$ is given by the scalar product of the $m$ th column with the $n$th column in $X^{*}$. The centered matrix $X^{*}$ contains the entries $-p$ and $1-p$. Thus, $\left(X^{* T} X^{*}\right)_{m n}$ contains products of the form $(1-p)^{2},(-p)^{2}$ and $-p(1-p)$. The shift between column $m$ and $n$ in $X^{*}$ is given by $\bmod \left(|m-n|, 2 N_{B L}\right)$. The scalar product of two columns $X_{m_{1}}^{*}$ and $X_{n_{1}}^{*}$ is the same as the scalar product of the columns $X_{m_{2}}^{*}$ and $X_{n_{2}}^{*}$ if the shift between column $m_{1}$ and $n_{1}$ is the same as the shift between column $m_{2}$ and column $n_{2}$. Thus for derivation of Eq. (5.49), it is sufficient to focus on the scalar product of the first column $X_{.1}^{*}$ of $X^{*}$ with all columns of $X^{*}$ (including the first column).

The first column of $X^{*}$ is the centered stimulus sequence $s^{*}$. As there are blocks of $A$ followed by blocks of $N$ in the fMRI experiment, there are also blocks of $(1-p)$ followed by blocks of $(-p)$ in the stimulus sequence $s^{*}$. A block of $(1-p)$ followed by a block of $(-p)$ in the stimulus sequence $s^{*}$ will also be called a cycle. The scalar product $s^{* T} s^{*}$ equals:

$$
N_{C} \cdot(1-p)^{2} \cdot N_{B L}+N_{C} \cdot(-p)^{2} \cdot N_{B L}
$$

Per shift of the centered stimulus sequence, the scalar product of $s^{*}$ and the $n$th column $X_{. n}^{*}$ looses one product of the form $(1-p)^{2}$ and one product of the form $(-p)^{2}$ and gains one product of the form $(-p)(1-p)$ in Eq. (5.50) per cycle. However, it has to be corrected for higher shift sizes when products of the form $(1-p)^{2}$ and $(-p)^{2}$ are regained and products of the form $(-p)(1-p)$ are lost again. By these considerations and observations the formula in Eq. (5.49) is obtained.

\section{B.2 Calculation for one stimulus and any block lengths}

In this section, blocked designs with one stimulus type will be considered without any assumption on block lengths. By $N_{B L_{0}}$ the number of trials in a null block will be denoted and by $N_{B L_{A}}$ the number of trials in a stimulus block will be denoted. It will be shown that

$$
\begin{aligned}
& \left(X^{* T} X^{*}\right)_{m n} \\
= & (1-p)^{2} \cdot\left(\max \left(N_{B L_{A}}-d, 0\right)+\max \left(0, d-N_{B L_{0}}\right)\right) \cdot N_{C} \\
+ & (-p)^{2} \cdot\left(\max \left(N_{B L_{0}}-d, 0\right)+\max \left(0, d-N_{B L_{A}}\right)\right) \cdot N_{C} \\
+ & 2(-p)(1-p) \cdot \min \left(d, N_{B L_{0}}+N_{B L_{A}}-d, N_{B L_{0}}, N_{B L_{A}}\right) \cdot N_{C}
\end{aligned}
$$

where $d=\bmod \left(|m-n|, N_{B L_{0}}+N_{B L_{A}}\right)$ gives the shift size between column $m$ and column $n$ in $X^{*}$. In Eq. (5.49) $2 N_{B L}$ has to be replaced with $N_{B L_{0}}+N_{B L_{A}}$ and $N_{B L}$ has to be 
replaced either with $N_{B L_{A}}$ or $N_{B L_{0}}$ to adapt Eq. (5.49) for unequal block length. With $d$ as defined above, we obtain:

$$
\begin{aligned}
& \left(X^{* T} X^{*}\right)_{m n} \\
= & (1-p)^{2} \cdot\left|N_{B L_{A}}-d\right| \cdot N_{C}+(-p)^{2} \cdot\left|N_{B L_{0}}-d\right| \cdot N_{C} \\
+ & 2(-p)(1-p) \min \left(d, N_{B L_{0}}+N_{B L_{A}}-d\right) \cdot N_{C} .
\end{aligned}
$$

In addition, other adaptations are necessary. It holds again that the scalar product of two columns $X_{. m_{1}}^{*}$ and $X_{. n_{1}}^{*}$ is the same as the scalar product of the columns $X_{. m_{2}}^{*}$ and $X_{. n_{2}}^{*}$ when the shift size of the two pairs is the same. Thus, we focus again on the scalar product of the first column $X_{.1}^{*}$ with the other columns in $X^{*}$. The scalar product of $X_{.1}^{*}$ with $X_{.1}^{*}$ equals:

$$
\begin{aligned}
& \left(X^{* T} X^{*}\right)_{11}=X_{.1}^{* T} X_{.1}^{*} \\
= & (1-p)^{2} \cdot N_{B L_{A}} \cdot N_{C}+(-p)^{2} \cdot N_{B L_{0}} \cdot N_{C} .
\end{aligned}
$$

Per cycle, the number of products of the form $(1-p)^{2}$ in the scalar product $\left(X^{* T} X^{*}\right)_{1 n}$ is given by

$$
\max \left(N_{B L_{A}}-d, 0\right)+\max \left(0, d-N_{B L_{0}}\right) .
$$

For explanation of Eq. (5.54), we assume in the following that $N_{B L_{0}}<N_{B L_{A}}$. For a shift size smaller than or equal to $N_{B L_{0}}$, one product of the form $(1-p)^{2}$ is lost per shift of the column $X_{. n}^{*}$ for each cycle. Thus, the number of products per cycle is totally explained by the loss of products given by $\left(N_{B L_{A}}-\right.$ shift size). When the shift size is between $N_{B L_{0}}$ and $N_{B L_{A}}$ the number of products is explained by a combination of loosing products and obtaining products as expressed by $\left(N_{B L_{A}}-\right.$ shift size $)+\left(\right.$ shift size $\left.-N_{B L_{0}}\right)$ when shifting. For a shift size higher than or equal to $N_{B L_{A}}$, the number of products is explained by the gain of products given by (shift size $-N_{B L_{0}}$ ). Generalizing these observations to a formula for all shift sizes leads to Eq. (5.54).

The same considerations as for the number of products of the form $(1-p)^{2}$ can be applied for the number of products of the form $(-p)^{2}$. Thus, the number of products of the form $(-p)^{2}$ is

$$
\left(\max \left(N_{B L_{0}}-d, 0\right)+\max \left(0, d-N_{B L_{A}}\right)\right) \cdot N_{C}
$$

There cannot be more products of the form $(1-p)(-p)$ per cycle than the smallest block length. Thus, the number of products of form $(1-p)(-p)$ per cycle is given by

$$
\min \left(d, N_{B L_{0}}+N_{B L_{A}}-d, N_{B L_{0}}\right) .
$$

If $N_{B L_{A}}<N_{B L_{0}}$, the same observations as above hold when $N_{B L_{A}}$ is replaced with $N_{B L_{0}}$ and vice versa. It follows Eq. (5.51) for all possible combinations of $N_{B L_{A}}$ and $N_{B L_{0}}$. The formula Eq. (5.51) reduces to Eq. (5.49) when $N_{B L_{0}}=N_{B L_{A}}$. Note that Eq. (5.51) also holds for $N_{B L_{0}}=0$ and the right half of Eq. (5.51) reduces then to $N_{C}(1-p)^{2} N_{B L_{A}}$. 


\section{B.3 Calculation for any number of stimulus types}

As before, the number of events in a stimulus block is denoted by $N_{B L_{A}}$ and the number of events in a null block is denoted by $N_{B L_{0}}$. The sequence $s^{q}$ is equal to a stimulus sequence $s^{\prime}$ with only one stimulus type and null events. The matrix $X^{\prime *}$ is the centered matrix for sequence $s^{\prime}$ and the product $X^{* T} X^{\prime *}$ is equal to $X_{q}^{* T} X_{q}^{*}$ for all stimuli $A_{1}, \ldots, A_{Q}$. As the length of null blocks in $s^{\prime}$ and $s^{q}$ is $(Q-1) N_{B L_{A}}+N_{B L_{0}}$ and the total length of one cycle $\left(A_{1} \ldots A_{Q} N\right)$ is $Q N_{B L_{A}}+N_{B L_{0}}$, we have to replace, $N_{B L_{0}}$ in Eq. (5.51) with $(Q-1) N_{B L_{A}}+N_{B L_{0}}$ and $N_{B L_{A}}+N_{B L_{0}}$ in Eq. (5.51) with $Q N_{B L_{A}}+N_{B L_{0}}$. We will use the notation $N_{B L_{0 T}}=(Q-1) N_{B L_{A}}+N_{B L_{0}}$ and $N_{B L_{T}}=Q N_{B L_{A}}+N_{B L_{0}}$ (T for total). Thus,

$$
\begin{aligned}
& \left(X_{q}^{* T} X_{q}^{*}\right)_{m n} \\
= & (1-p)^{2} \cdot\left(\max \left(N_{B L_{A}}-d, 0\right)+\max \left(0, d-N_{B L_{0 T}}\right)\right) \cdot N_{C} \\
+ & (-p)^{2} \cdot\left(\max \left(N_{B L_{0 T}}-d, 0\right)+\max \left(0, d-N_{B L_{A}}\right)\right) \cdot N_{C} \\
+ & 2(-p)(1-p) \min \left(d, N_{B L_{T}}-d, N_{B L_{0 T}}\right) \cdot N_{C}
\end{aligned}
$$

where $d=\bmod \left(|m-n|, N_{B L_{T}}\right)$ is the shift size between column $m$ and $n$ in $X_{q}^{*}$. In the next step, we need to determine what the off-diagonal products of $X^{* T} X^{*}$ in Eq. (5.28) are. As block lengths are equal for all stimulus blocks, sequence $s^{q}$ is only a shifted version of $s^{1}$. The shift is given by $(q-1) N_{B L_{A}}$. The shift between column $m$ in $X_{1}^{*}$ and column $n$ in $X_{q}^{*}$ is thus given by $(q-1) N_{B L_{A}}+(m-n)$. The product $X_{1}^{* T} X_{q}^{*}$ is as in Eq. (5.57) but $|m-n|$ is replaced by $(q-1) N_{B L_{A}}+(m-n)$. Due to the symmetry of $X^{* T} X^{*}$ it holds that $X_{q}^{* T} X_{1}^{*}=X_{1}^{* T} X_{q}^{*}$. We determined the products $X_{1}^{* T} X_{q}^{*}$ and $X_{q}^{* T} X_{1}^{*}$ but we still need to determine the products $X_{q_{1}}^{* T} X_{q_{2}}^{*}$ for $q_{1} \neq 1$ and $q_{2} \neq 1$.

Because of the symmetry of $X^{* T} X^{*}$ we will focus on $q_{1}<q_{2}$. The shift between sequence $s^{q_{1}}$ and $s^{q_{2}}$ is the same as the shift between sequence $s^{1}$ and sequence $s^{q_{2}-q_{1}+1}$. The shift is given by $\left(q_{2}-q_{1}\right) N_{B L_{A}}$. Thus, the shift between column $m$ in $X_{q_{1}}^{*}$ and column $n$ in $X_{q_{2}}^{*}$ is given by $\left(q_{2}-q_{1}\right) N_{B L_{A}}+(m-n)$. The product $X_{q_{1}}^{* T} X_{q_{2}}^{*}$ is thus as in Eq. (5.57) but we need to replace $|m-n|$ with $\left|\left(q_{2}-q_{1}\right) N_{B L_{A}}+(m-n)\right|$. Because of the symmetry of $X^{* T} X^{*}$ it holds that $X_{q_{1}}^{* T} X_{q_{2}}^{*}=X_{q_{2}}^{* T} X_{q_{1}}^{*}$. It follows Eq. (5.29) for general $q_{1}$ and $q_{2}$. Note that this result also holds for $N_{B L_{0}}=0$ and of course for $N_{B L_{A}}=N_{B L_{0}}$. 


\section{CHAPTER 6}

Summary and recommendations 


\subsection{Summary}

In this thesis optimal experimental designs for functional magnetic resonance imaging are presented. A design is called optimal if it is evaluated best by a criterion expressing the requirements which a researcher has on the design, e.g., low variances for stimulus effects estimators or a desired stimulus frequency. Several requirements can be combined in a multi-objective design criterion.

In the introductory Chapter 1 the underlying models for the main design criteria, i.e., detection of activation and estimation of HRF, and the design criteria themselves are explained. For detection power a single-subject and a multi-subject model are presented while for estimation of HRF a linear and a nonlinear single-subject model are given. It is described how designs can be evaluated by the $A$ - or $D$-optimality criterion on their ability to precisely estimate effects of interest, e.g., stimulus effects or the contrasts of stimulus effects for detection power. Furthermore, Chapter 1 discusses the main types of experimental fMRI designs: blocked and event-related designs.

In Chapters $\mathbf{2}$ and $\mathbf{5}$ the focus is on the optimization of blocked designs for detection power in a single-subject analysis and multi-subject analysis, respectively. In Chapter 2 blocked designs with fixed total duration are optimized with respect to the SOA, block length and block order. Similar (locally) optimal designs and maximin designs were found for different error structures, i.e., uncorrelated errors (UE), AR1 and AR1+ME. The error structure only had an effect on the maximin block length and optimal block length. All maximin designs (AR1,AR1+ME) or optimal designs (UE) had SOA equal to $1 \mathrm{~s}$ and the block order depended on the optimality criterion: ANBN for the $A$-optimality criterion, ABN for the $D_{-}, D_{S^{-}}$and $A_{S^{-}}$optimality criterion and $\mathrm{AB}$ for the $c$-optimality criterion. The maximin designs for the AR1 error structure all had high maximin values and were thus highly efficient against all locally optimal designs. The maximin block length was $15 \mathrm{~s}$ for AR1 and all optimality criteria whereas for uncorrelated errors the optimal block length was $15 \mathrm{~s}$ or $20 \mathrm{~s}$ and for AR $1+\mathrm{ME}$ the maximin block length was $10 \mathrm{~s}$ or $15 \mathrm{~s}$.

In Chapter 5 the optimal number of subjects and the optimal number of cycles (repetitions) of $A_{1} \ldots A_{Q} N$, consisting of task blocks $A$ for $Q$ stimulus types and null blocks $N$, in a blocked design with limited study budget are studied. Analytical results are presented based on a linear mixed-effects model with uncorrelated errors and compared to numerical results for a model with correlated errors in an AR1 form. It was seen that the $A$-optimal number of cycles increased for correlated errors in comparison to uncorrelated errors whereas the $A$-optimal number of subjects decreased for correlated errors. In the analytical formulae and numerical results it was shown that the $A$-optimal number of cycles and the $A$-optimal number of subjects depended on the within- to between-subject variance ratio.

Maximin designs for detection power, estimation efficiency and multi-objective criteria based on $A$ - or $D$-optimality are discussed in Chapter 3 for a possible range of autocorrelation in an AR1 error model. The maximin designs were chosen from the locally optimal designs for a given value of autocorrelation. The determined maximin designs were robust against misspecification of the autocorrelation due to a high maximin value, and were obtained as locally optimal designs for a medium value of autocorrelation. Under 
the assumption of uncorrelated errors, the $D$-optimal stimulus frequency for estimation of stimulus effects (detection power) and estimation of HRF (estimation efficiency) was analytically derived to be equal to $1 /(Q+1)$, where $Q$ was the number of stimulus types. For estimation of contrasts between stimulus effects or HRFs of different stimulus types, the $D$-optimal stimulus frequency was analytically shown to be equal to $1 / Q$ for uncorrelated errors. It was reported that the stimulus frequencies of the $D$-maximin designs under AR1 were close to the analytical $D$-optimal stimulus frequency under the assumption of uncorrelated errors. Maximin block lengths were slightly shorter than in Chapter 2, i.e., 10 s to $14 \mathrm{~s}$ ( $A$-optimality) and $11 \mathrm{~s}$ to $12 \mathrm{~s}$ ( $D$-optimality), which is likely due to the bigger design space in Chapter 3.

Chapter 4 is devoted to the optimization of designs for nonlinear estimation of the double gamma function. It was shown that the maximin design, which was chosen from locally optimal designs obtained by a genetic algorithm for different parameter HRF values, performed best in comparison with other design types. The other design types encompassed blocked designs, slow event-related designs, $m$-sequences and rapid event-related designs with a geometric or uniform distribution for ISI. An optimal design for nonlinear estimation of the double gamma function seemed to be between an event-related design and a blocked design. The optimal design for linear estimation also performed well for nonlinear estimation efficiency whereas the optimal design for detection power was not very efficient for nonlinear estimation of the HRF.

\subsection{Guidelines}

Which design is optimal, depends heavily on the effects of interest in the analysis model and to a lesser amount on the optimality criterion, i.e., $A$ - or $D$-optimality criterion. Generally, it is therefore recommended that an fMRI researcher carefully decides on the experimental purpose and how to conduct it before the experiment. While such thoughts before conducting an experiment are of course important for any kind of experiment, they are perhaps even more important for fMRI experiments due to the complexity of decisions and the high costs (Culham, 2006). In the following, we will discuss the recommendations for the different parameters of interest and optimality criteria separately.

For detection of individual stimulus effects, the optimal design is a blocked design. In some situations, e.g., for post-hoc sorting of trials, an event-related design has to be performed but we will focus in the following on the optimal properties of such a blocked design. The optimal block order is ABN, i.e., null blocks (rest or baseline condition) happening equally often as stimulus blocks. The time between trials in one block should be as short as possible and the block length should be chosen between $10 \mathrm{~s}$ and $20 \mathrm{~s}$. For the $A$-optimality criterion, block lengths for null blocks should be longer than for stimulus blocks whereas for the $D$-optimality criterion null blocks should have the same length as stimulus blocks. For detection of contrasts between stimuli types, the block order has to be changed to $\mathrm{AB}$, i.e., no null blocks.

When the interest is in linear estimation of the HRF, a rapid event-related design should be applied. We recommend to apply the genetic algorithm to obtain an optimal design. 
As for detection power, the $D$ - and $A$-optimal design differ in the optimal frequencies for stimulus types and null events. For the $D$-optimal design, null events should happen equally often as each stimulus type, thus the $D$-optimal stimulus frequency $p_{D}$ equals $1 /(Q+1)$, where $Q$ is the number of stimulus types. In contrast, the $A$-optimal stimulus frequency $p_{A}$ equals $1 /(Q+\sqrt{Q})$ which implies that each stimulus type happens less often than null events. For estimation of contrasts between HRFs of different stimulus types, no null events should be applied for the $A$-optimality criterion and $D$-optimality criterion. If the genetic algorithm is applied, these optimal frequencies will be automatically obtained.

To apply the genetic algorithm for estimation efficiency, detection power, counterbalancing, maintenance of desired stimulus frequency or a multi-objective criterion, a maximin approach can be used considering a range of possible autocorrelation parameters in an AR1 model. It is however also possible to use a medium value of autocorrelation as the maximin designs were obtained for medium values and locally optimal designs for a medium value of autocorrelation were efficient. Only one run of the genetic algorithm is then necessary.

For nonlinear estimation of the HRF, the genetic algorithm adapted for nonlinear HRF estimation is recommendable to determine a maximin design from the locally optimal designs obtained by the GA for different HRF parameter values. If the computational effort of this approach is too high in a certain situation, determination of the maximin $m$ sequence is computationally more efficient and statistically almost as efficient as the GA maximin design. Further, if no $m$-sequence is available for the given number of stimulus types, efficient alternatives are rapid event-related designs with a geometric distribution for the ISI or an optimal design for linear estimation of the HRF.

Before conducting a multi-subject blocked fMRI experiment with detection of activated areas as the aim, the formulae presented in Chapter 5 or our MATLAB code for correlated errors can be applied to determine the optimal number of subjects and cycles. This demands from the researcher to prespecify the within- to between-subject variance ratio from a pilot study or from previous studies.

\subsection{Ideas for future research}

In this thesis we extended previous work from other researchers by considering different correlated error structures, i.e., AR1 and AR1+ME, and different values for the error parameters. Furthermore, we considered in Chapters 3, 4 and 5 a nuisance matrix with Legendre polynomials of order 2 or a discrete cosine transform (DCT) set. However, other aspects of noise could further be modeled within the nuisance matrix and their effect on the optimal design could be determined, e.g., physiological noise due heart beat and breathing. Heterogeneity of the error variance seems to have been neglected in the analysis of fMRI data (Huettel et al., 2004, p.342) as well as for the optimization of experimental fMRI design. However, Huettel et al. (2001) showed that variability in the fMRI signal near the peak of the HRF is higher than for prestimulus baseline. This finding indicates that a heterogeneous error variance with higher variance during stimulus conditions than during the rest condition might be justified.

Another aspect which has been neglected for the optimization of fMRI experiments 
is the influence of a fixed hemodynamic response function on the results for detection power. A different form of the hemodynamic response function will lead to a different power spectrum of the HRF and as a consequence to different optimal designs for detection power, e.g., different optimal block lengths. A maximin criterion can be used to find designs which are efficient for all possible values of the parameters in the double gamma function. Furthermore, Bayesian modeling of the hemodynamic response function parameters (Gössl et al., 2001) can be applied to obtain Bayesian optimal designs for detection power.

Another idea for possible research considers estimation efficiency. For simplicity, we focused in this thesis mainly on designs where the SOA is a multiple of TR. However, it might also be interesting to study event-related designs where the SOA is no multiple of the TR. Rapid event-related designs can be generated by using a minimum SOA ( $\left.\mathrm{SOA}_{\text {min }}\right)$ and the probability $p$ of each stimulus type and null events to occur at time points separated by $\mathrm{SOA}_{\text {min }}$. An $\mathrm{SOA}_{\text {min }}$ which is not a multiple of $\mathrm{TR}$, results in a higher sampling rate for the HRF. However, an $\mathrm{SOA}_{\text {min }}$ higher than TR also results in fewer trials than a design with $\mathrm{SOA}_{\text {min }}$ equal to TR assuming that the total experimental duration is fixed. As a consequence, the heights of the HRF at the different time points are not estimated as efficiently as in the design with $\mathrm{SOA}_{\min }$ equal to TR. This trade-off in number of time points for HRF estimation and efficiency of HRF estimation corresponds to a trade-off in resolution and precision of HRF estimation and should be investigated.

As an extension of Chapter 5, it would be interesting to study further the effect of autocorrelation in the AR1 structure on the $A$-optimal number of cycles and subjects. By numerical calculation of the $A$-optimal number of cycles for different autocorrelation values $\rho$, it can be studied whether a factor $f(\rho)$ can be found so that $f(\rho)$ times the $A$-optimal number of cycles for uncorrelated errors equals the $A$-optimal number of cycles for correlated errors with autocorrelation $\rho$. It is expected that such a factor would also depend on the cycle length as longer cycles would be less correlated with each other so that the $A$-optimal number of cycles for correlated errors would be closer to the $A$-optimal number of cycles for uncorrelated errors. Similarly, such a factor can be searched for the $A$-optimal number of subjects. It might also be possible to obtain analytical results for the $A$-optimal number of subjects and cycles under the assumption of correlated errors. Furthermore, application of the $D$-optimality criterion and estimation of contrasts between stimulus types can be investigated.

In Chapter 5 the subject level was added to the model and as an aspect of optimization. However, other levels are also commonly employed and need to be studied, i.e., multiple runs and multiple scanning sessions. A first study on optimal design for multiple scanning sessions has been performed by Kao et al. (2009) but more research is needed. On the subject level, it might further be relevant to study the optimal number of subjects for different groups, e.g., healthy subjects or patients with a certain disease. 
CHAPTER 7

Samenvatting en richtlijnen (Dutch summary) 


\subsection{Samenvatting}

In dit proefschrift worden optimale experimentele designs (Nederlands: proefopzetten) voor functionele MRI (fMRI) gepresenteerd. Een design wordt optimaal genoemd als het design het beste presteert op een criterium dat de eisen uitdrukt, die een onderzoeker stelt aan het design. Voorbelden van eisen zijn een lage variantie voor de schatter van stimuluseffecten en een gewenste stimulusfrequentie. Verschillende eisen kunnen worden gecombineerd in een multi-objectief design criterium.

In het inleidende Hoofdstuk 1 worden de belangrijkste designcriteria toegelicht, namelijk detectie van activering (detectievermogen) en de schatting van de hemodynamische responsfunctie (HRF), en worden de onderliggende modellen gepresenteerd. Voor de detectie van activatie worden een single-subject model en een multi-subject model gepresenteerd, terwijl voor de schatting van de HRF een lineair en een niet-lineair singlesubject-model worden gegeven. Er wordt beschreven hoe designs middels het $A$ - of $D$ optimaliteitscriterium kunnen worden beoordeeld op hun vermogen om nauwkeurig de effecten te schatten, waarin de onderzoeker geïnteresseerd is, bijvoorbeeld stimuluseffecten of contrasten van stimuluseffecten. Bovendien gaat Hoofdstuk 1 in op de belangrijkste vormen van experimentele fMRI-designs: blocked en event-related designs.

In de Hoofdstukken $\mathbf{2}$ en $\mathbf{5}$ ligt de focus op de optimalisatie van blocked designs voor het detectievermogen in respectievelijk een single-subject analyse en multi-subject analyse. In Hoofdstuk 2 worden blocked designs met vaste totale duur geoptimaliseerd met betrekking tot de stimulus onset asynchrony (SOA), bloklengte en blokvolgorde. Soortgelijke (lokaal) optimale designs en maximin designs worden gevonden voor verschillende covariantiestructuren van de ruis of toevallige fout in de data, namelijk ongecorreleerde fouten (uncorrelated errors, UE), eerste-orde autoregressieve fout (AR1) en eerste-orde autoregressieve fout plus meetfout (first-order autoregressive error plus measurement error, $\mathrm{AR} 1+\mathrm{ME})$. De foutstructuur heeft alleen een effect op de maximin bloklengte en de optimale bloklengte. Alle maximin designs (AR1, AR1+ME) of optimale designs (UE) hebben een SOA van $1 \mathrm{sec}$ en een blokvolgorde afhankelijk van het optimaliteitscriterium: ANBN voor het $A$-criterium, ABN voor de $D$-, $D_{S^{-}}$en $A_{S^{-}}$criteria en $\mathrm{AB}$ voor het $c$-criterium. De maximin designs voor de AR1-foutstructuur hebben alle hoge maximin waarden en zijn dus zeer efficiënt vergeleken met de lokaal optimale designs. De maximin bloklengte voor AR1 en alle optimalisatiecriteria is $15 \mathrm{sec}$, terwijl de optimale bloklengte voor ongecorreleerde fouten $15 \mathrm{sec}$ of $20 \mathrm{sec}$ is en de maximin bloklengte voor AR1+ME $10 \mathrm{sec}$ of $15 \mathrm{sec}$ is.

In Hoofdstuk 5 worden het optimale aantal proefpersonen en het optimale aantal cycli (repetities) $A_{1} \ldots A_{Q} N$ van taakblokken $(A)$ voor $Q$ stimulustypen en een nulblok $(N)$ bij een blocked design met een beperkt studiebudget onderzocht. Analyseresultaten worden gepresenteerd op basis van een lineair mixed-effects model met ongecorreleerde fouten, en vergeleken met numerieke resultaten voor een model met gecorreleerde fouten van het AR1-type. Het blijkt dat het $A$-optimale aantal cycli voor gecorreleerde fouten hoger is dan voor ongecorreleerde fouten, en het $A$-optimale aantal proefpersonen daardoor lager is vanwege de budgetrestrictie. Uit de analytische formules en numerieke resultaten blijkt voorts dat het $A$-optimale aantal cycli en het $A$-optimale aantal proefpersonen afhangen van de verhouding tussen de binnen-subject en tussen-subject varianties. 
In Hoofdstuk 3 worden maximin designs voor het detectievermogen, schattingsefficiëntie voor de HRF en multi-objectief criteria gebaseerd op $A$ - of $D$-optimaliteit voor diverse mogelijke waarden van autocorrelatie in een single-subject model met AR1-fout besproken. De maximin designs zijn uit de lokaal optimale designs voor een gegeven waarde van autocorrelatie gekozen. De vastgestelde maximin designs zijn op grond van een hoge maximin waarde robuust tegen misspecificatie van de autocorrelatie, en komen overeen met lokaal optimale designs voor een gemiddelde waarde van autocorrelatie. Onder de aanname van ongecorreleerde fouten is de $D$-optimale stimulusfrequentie voor de schatting van de stimuluseffecten (detectievermogen) en de schatting van de HRF (schattingsefficiëntie) analytisch afgeleid en gelijk aan 1/( $Q+1)$, waarbij $Q$ het aantal van de stimulustypen is. Voor de schatting van de contrasten tussen stimuluseffecten of HRFs van verschillende stimulustypen, is de $D$-optimale stimulusfrequentie analytisch gelijk aan $1 / Q$ voor ongecorreleerde fouten. Het wordt voorts getoond dat de stimulusfrequenties van de $D$-maximin designs onder AR1 dichtbij de analytische $D$-optimale stimulusfrequentie voor ongecorreleerde fouten liggen. Maximin bloklengten zijn iets korter dan in Hoofdstuk 2, dat wil zeggen, 10 sec tot $14 \mathrm{sec}$ ( $A$-optimaliteit) en $11 \mathrm{sec}$ tot $12 \mathrm{sec}$ ( $D$-optimaliteit), wat waarschijnlijk te wijten is aan de grotere designruimte in Hoofdstuk 3.

Hoofdstuk 4 is gewijd aan de optimalisering van designs voor niet-lineaire schatting van de dubbele gamma-functie, een bekend model voor de HRF. Met een genetisch algoritme (GA) worden lokaal optimale designs voor verschillende waarden van de parameters in de dubbele gamma-functie gevonden. Vervolgens wordt het maximin design $\mathrm{MMD}_{\mathrm{GA}}$ uit deze lokaal optimale designs gekozen. Het is aangetoond dat dit maximin design $\mathrm{MMD}_{\mathrm{GA}}$ beter presteert dan de maximin designs van andere designsoorten. Deze andere designsoorten omvatten blocked designs, slow event-related designs, $m$-sequenties en rapid eventrelated designs met een geometrische of uniform verdeling voor het interstimulus interval (ISI). Een optimaal design voor niet-lineaire schatting van de dubbele gamma-functie lijkt tussen een event-related design en een blocked design te liggen. Het optimale design voor lineaire schatting van de HRF presteert ook goed voor niet-lineaire schatting, terwijl het optimale design voor detectievermogen niet erg efficiënt is voor niet-lineaire schatting.

\subsection{Richtlijnen}

Welk design optimaal is, hangt sterk af van de effecten die van belang zijn in het analysemodel, en in mindere mate van het optimaliteitscriterium, dat wil zeggen, $A$ - of $D$ optimaliteitscriteria. In het algemeen is het daarom aanbevolen dat een fMRI-onderzoeker vooraf zorgvuldig besluit over het experimentele doel en de uitvoering van het experiment. Hoewel dergelijke zaken natuurlijk van belang zijn voor elke vorm van experiment, zijn ze wellicht nog belangrijker voor de fMRI-experimenten. Dit hangt samen met de complexiteit van de beslissingen en de hoge kosten (Culham, 2006). Hieronder worden de aanbevelingen voor de verschillende parameters van belang en optimaliteitscriteria afzonderlijk besproken.

Voor de detectie van individuele stimuluseffecten is het optimale design een blocked design. In sommige situaties, bijvoorbeeld bij het post-hoc sorteren van de evenementen, is een event-related design nodig, maar we zullen ons hierna richten op de optimale eigen- 
schappen van een blocked design. De optimale blokvolgorde is $\mathrm{ABN}$, dat wil zeggen dat nulblokken (rust of referentietoestand) even vaak voorkomen als stimulusblokken (taakblokken). De tijd tussen de evenementen in een blok moet zo kort mogelijk zijn. De bloklengte moet tussen 10 en 20 seconden zijn. Voor het $A$-optimaliteitscriterium mogen bloklengten voor nulblokken langer dan stimulusblokken zijn, terwijl voor het $D$-optimaliteitscriterium nulblokken dezelfde lengte als stimulusblokken moeten hebben. Voor de detectie van contrasten tussen stimulustypen moet de blokvolgorde worden veranderd in $\mathrm{AB}$, dat wil zeggen geen nulblokken.

Wanneer men geïnteresseerd is in lineaire schatting van de HRF, moet een rapid eventrelated design worden toegepast. Wij raden aan om het genetische algoritme toe te passen om een optimaal design te verkrijgen. Zoals voor het detectievermogen verschillen de $D$ - en $A$-optimale designs in optimale frequenties voor stimulustypen en nulevenementen. Voor het $D$-optimale design, moeten nulevenementen even vaak gebeuren als voor elk stimulustype, waardoor de $D$-optimale stimulusfrequentie $p_{D}$ gelijk is aan $1 /(Q+1)$, waarbij $Q$ het aantal stimulustypen is. De $A$-optimale stimulusfrequentie $p_{A}$ daarentegen is gelijk aan $1 /(Q+\sqrt{Q})$, wat betekent dat elk stimulustype minder vaak voorkomt dan nulevenementen. Bij de schatting van de contrasten tussen HRFs van verschillende stimulustypen mogen geen nulevenementen worden toegepast voor het $A$ - en $D$-optimaliteitscriterium. Als het genetische algoritme wordt toegepast, worden deze optimale frequenties automatisch verkregen.

Voor de toepassing van het genetische algoritme voor de schattingsefficiëntie, het detectievermogen, counterbalancing, het onderhoud van de gewenste stimulusfrequentie of een multi-objectief criterium kan een maximin methode voor een interval van mogelijke autocorrelatieparameters in een AR1-model worden gebruikt. Ook is het mogelijk om een gemiddelde waarde van de autocorrelatie te gebruiken, omdat de maximin designs als lokaal optimale designs voor middelgrote waarden werden verkregen en de lokaal optimale designs voor een gemiddelde waarde van de autocorrelatie efficiënt waren. Slechts één run van het genetisch algoritme is dan noodzakelijk.

Voor niet-lineaire schatting van de HRF is het genetisch algoritme aangepast voor niet-lineaire HRF-schatting aan te bevelen. In dat geval kan een maximin design worden bepaald uit de lokaal optimale designs die verkregen zijn door het GA voor verschillende HRF-parameterwaarden. Als de rekentijd van deze aanpak in een bepaalde situatie te hoog is, is de bepaling van de maximin $m$-sequentie computationeel efficiënter en statistisch bijna net zo efficiënt als het GA maximin design. Bovendien geldt dat als geen $m$-sequentie voor het gegeven aantal stimulustypen beschikbaar is, rapid event-related designs met een geometrische verdeling voor het ISI en een optimaal design voor lineaire schatting van de HRF efficiënte alternatieven zijn.

Om een multi-subject blocked fMRI-experiment met detectie van actieve gebieden als doel uit te voeren, kunnen de formules in Hoofdstuk 5 voor ongecorreleerde fouten of onze MATLAB code voor gecorreleerde fouten worden toegepast om het optimale aantal proefpersonen en cycli te bepalen. Dit vraagt van de onderzoeker een specificatie van de verhouding binnen- en tussen-subject variantie, welke kan worden bepaald aan de hand van een pilot-studie of eerdere studies. 


\subsection{Ideeën voor toekomstig onderzoek}

Het in dit proefschrift gepresenteerde onderzoek is in minstens twee opzichten een uitbreiding van eerder onderzoek: in het hier gepresenteerde onderzoek zijn gecorreleerde foutstructuren gebruikt ( $\mathrm{AR} 1$ en $\mathrm{AR} 1+\mathrm{ME}$ ) en voor de foutstructuur zijn verschillende waarden gebruikt. Verder is in Hoofdstukken 3, 4 en 5 rekening gehouden met ruis, namelijk door toepassing van een nuisance matrix met Legendre-polynomen van orde 2 of een discrete cosine transform set. Binnen de nuisance matrix kunnen ook andere aspecten van ruis (bijvoorbeeld fysiologische ruis veroorzaakt door hartslag en ademhaling) worden gemodelleerd en kan hun effect op het optimale design worden bepaald. Heterogeniteit van de foutvariantie lijkt te zijn geïgnoreerd in de analyse van de fMRI-data (Huettel et al., 2004, p.342) evenals voor de optimalisatie van het experimentele fMRI-design. Huettel et al. (2001) hebben laten zien dat de variabiliteit in de fMRI-signaal in de buurt van de HRF-piek hoger is dan voor prestimulus baseline. Deze bevinding geeft aan dat een heterogene fout met een hogere variantie tijdens stimulus condities dan in de rest condities kan worden gerechtvaardigd.

Een ander aspect dat niet uitgebreid onderzocht is voor de optimalisatie van fMRIexperimenten is de invloed van een vaste hemodynamische responsfunctie op de resultaten voor het detectievermogen. Veranderingen in vorm van de hemodynamische responsfunctie leiden tot veranderingen in het powerspectrum van de HRF. Gevolg daarvan kan een ander optimaal design voor het detectievermogen zijn, bijvoorbeeld andere optimale bloklengten. Een maximin criterium kan worden gebruikt om designs te vinden die efficiënt zijn voor alle mogelijke waarden van de parameters in de dubbele gamma-functie. Bovendien kan Bayesiaanse modellering van de hemodynamische responsfunctieparameters (Gössl et al., 2001) worden toegepast voor het verkrijgen van Bayesiaanse optimale designs voor het detectievermogen.

Een ander idee voor een mogelijk onderzoek gaat over schattingsefficiëntie. Omwille van de eenvoud heeft het onderzoek gepresenteerd in dit proefschrift zich met name gericht op modellen waarin de SOA een veelvoud van TR is. Het bestuderen van event-related designs waarin de SOA géén veelvoud van de TR is, is mogelijk ook interessant. Rapid event-related designs kunnen met behulp van een minimum SOA $\left(\mathrm{SOA}_{\text {min }}\right)$ en de kans $p$ worden gegenereerd. De kans $p$ geeft de kans aan van elk stimulustype en nulevenement op de tijdstippen die van elkaar gescheiden zijn door de $\mathrm{SOA}_{\min }$. Een $\mathrm{SOA}_{\text {min }}$, die niet een veelvoud is van TR, resulteert in een hogere sampling rate voor de HRF. Echter, een $\mathrm{SOA}_{\text {min }}$ hoger dan $\mathrm{TR}$ resulteert ook in minder evenementen dan een design met $\mathrm{SOA}_{\text {min }}$ gelijk aan TR in de veronderstelling dat de totale experimentele duur vastgesteld is. Als gevolg hiervan zijn de hoogtes van de HRF op de verschillende tijdstippen niet zo efficiënt geschat als in het design met $\mathrm{SOA}_{\text {min }}$ gelijk aan TR. Deze trade-off in het aantal tijdstippen voor HRF-schatting en de efficiëntie van HRF-schatting komt overeen met een trade-off in de resolutie en nauwkeurigheid van de HRF-schatting en dient te worden onderzocht.

Als een uitbreiding van Hoofdstuk 5 zou het interessant zijn om het effect van autocorrelatie in de AR1-structuur op het $A$-optimale aantal cycli en het $A$-optimale aantal proefpersonen verder te bestuderen. Door numerieke berekening van het $A$-optimale aantal cycli voor verschillende autocorrelatiewaarden $\rho$ kan worden onderzocht of een factor $f(\rho)$ 
kan worden vastgesteld zo dat $f(\rho)$ maal het $A$-optimale aantal cycli voor ongecorreleerde fouten gelijk is aan het $A$-optimale aantal cycli voor gecorreleerde fouten met autocorrelatie $\rho$. De verwachting is dat een dergelijke factor mede afhankelijk is van de cycluslengte, aangezien langere cycli minder sterk met elkaar gecorreleerd zijn dan kortere cycli, zodat het $A$-optimale aantal cycli voor gecorreleerde fouten dichterbij het A-optimale aantal cycli voor ongecorreleerde fouten ligt. Evenzo kan een dergelijke factor worden gezocht voor het $A$-optimale aantal proefpersonen. Het zou ook mogelijk zijn om analytische resultaten te verkrijgen voor het $A$-optimale aantal proefpersonen en cycli in de veronderstelling van gecorreleerde fouten. Tot slot zouden ook toepassing van het $D$-optimaliteitscriterium en de schatting van contrasten tussen stimulustypen kunnen worden onderzocht.

In Hoofdstuk 5 is het proefpersoonniveau als een optimalisatieaspect aan het model toegevoegd. Ook andere veelvuldig gebruikte niveaus verdienen nader onderzoek. Te denken valt hierbij aan meerdere runs en meerdere scannersessies. Een eerste studie naar het optimale design voor meerdere scannersessies is uitgevoerd door Kao et al. (2009) maar verder onderzoek is nodig. Voor het proefpersoonniveau kan het verder relevant zijn om het optimale aantal proefpersonen voor verschillende groepen te bestuderen, bijvoorbeeld, gezonde proefpersonen of patiënten met een bepaalde ziekte. 


\section{References}

Aguirre, G. K. \& D'Esposito, M. (1999). Experimental design for brain fMRI. In C. T. W. Moonen \& P. A. Bandettini (Eds.), Functional MRI (pp. 369-380). Berlin: Springer.

Aguirre, G. K., Zarahn, E., \& D'Esposito, M. (1998). The variability of human, BOLD hemodynamic responses. NeuroImage, 8, 360-369.

Amaro, E. J. \& Barker, G. J. (2006). Study design in fMRI: Basic principles. Brain and Cognition, 60, 220-232.

Atkinson, A. C. \& Donev, A. N. (1996). Optimum experimental designs. Oxford: Clarendon Press.

Atkinson, A. C., Donev, A. N., \& Tobias, R. D. (2007). Optimum experimental designs with $S A S$. Oxford: Oxford University Press.

Bandettini, P. A. \& Cox, R. W. (2000). Event-related fMRI contrast when using constant interstimulus interval: Theory and experiment. Magnetic Resonance in Medicine, 43, $540-548$.

Bandettini, P. A. \& Underleider, L. G. (2001). From neuron to BOLD: New connections. Nature Neuroscience, 4(9), 864-866.

Beckmann, C. F., Jenkinson, M., \& Smith, S. M. (2001). General multilevel linear modelling for group analysis in FMRI. Technical report, Oxford Centre for Functional Magnetic Resonance Imaging of the Brain (FMRIB), http://www.fmrib.ox.ac.uk/analysis/techrep/.

Belliveau, J. W., Kennedy, D. N., McKinstry, R. C., Buchbinder, B. R., Weisskoff, R. M., Cohen, M. S., Vevea, J. M., Brady, T. J., \& Rosen, B. R. (1991). Functional mapping of the human visual cortex by magnetic resonance imaging. Science, 254(5032), 716-719.

Berger, M. P. F. \& Tan, F. E. S. (2004). Robust designs for linear mixed effects models. Applied Statistics, 53(4), 569-581. 
Bianciardi, M., Cerasa, A., Patria, F., \& Hagberg, G. E. (2004). Evaluation of mixed effects in event-related fMRI studies: Impact of first-level design and filtering. NeuroImage, 22, 1351-1370.

Binder, J. R., Rao, S. M., Hammeke, T. A., Frost, J. A., Bandettini, P. A., \& Hyde, J. S. (1994). Effects of stimulus rate on signal response during functional magnetic resonance imaging of auditory cortex. Cognitive Brain Research, 2, 31-38.

Birn, R. M., Cox, R. W., \& Bandettini, P. A. (2002). Detection versus estimation in event-related fMRI: Choosing the optimal stimulus timing. NeuroImage, 15, 252-264.

Boynton, G. M., Engel, S. A., Glover, G. H., \& Heeger, D. J. (1996). Linear systems analysis of functional magnetic resonance imaging in human V1. The Journal of Neuroscience, 16(13), 4207-4221.

Buckner, R. L., Bandettini, P. A., O'Craven, K. M., Savoy, R. L., Petersen, S. E., Raichle, M. E., \& Rosen, B. R. (1996). Detection of cortical activation during averaged single trials of a cognitive task using functional magnetic resonance imaging. Proceedings of the National Academy of Sciences, USA, 93, 14878-14883.

Bullmore, E., Brammer, M., Williams, S. C. R., Rabe-Hesketh, S., Janot, N., David, A., Mellers, J., Howard, R., \& Sham, P. (1996). Statistical methods of estimation and inference for functional MR image analysis. Magnetic Resonance in Medicine, 35, 261277.

Buračas, G. T. \& Boynton, G. M. (2002). Efficient design of event-related fMRI experiments using $m$-sequences. NeuroImage, 16, 801-813.

Burock, M. A., Buckner, R. L., Woldorff, M. G., Rosen, B. R., \& Dale, A. M. (1998). Randomized event-related designs allow for extremely rapid presentation rates using functional MRI. NeuroReport, 9, 3735-3739.

Burock, M. A. \& Dale, A. M. (2000). Estimation and detection of event-related fMRI signals with temporally correlated noise: A statistically efficient and unbiased approach. Human Brain Mapping, 11, 249-260.

Buxton, R. B., Uludağ, K., Dubovitz, D. J., \& Liu, T. T. (2004). Modeling the hemodynamic response to brain activation. NeuroImage, 23, S220-S233.

Chaloner, K. \& Verdinelli, I. (1995). Bayesian experimental design: A review. Statistical Science, $10(3), 273-304$.

Chatfield, C. (2004). The analysis of time series: An introduction (6th ed.). Boca Raton, FL: Chapman \& Hall/CRC.

Chee, M. W., Venkatraman, V., Westphal, C., \& Siong, S. C. (2003). Comparison of block and event-related fMRI designs in evaluating the word-frequency effect. Human Brain Mapping, 18, 186-193. 
Chein, J. M. \& Schneider, W. (2003). Designing effective fMRI experiments. In J. Grafman \& I. H. Robertson (Eds.), Handbook of neuropsychology: Vol. 9. Plasticity and rehabilitation (2nd ed., pp. 299-325). Amsterdam: Elsevier.

Ciuciu, P., Poline, J.-B., Marrelec, G., Idier, J., Pallier, C., \& Benali, H. (2003). Unsupervised robust nonparametric estimation of the hemodynamic response function for any fMRI experiment. IEEE Transactions on medical imaging, 22(10), 1235-1251.

Cohen, M. S. (1997). Parametric analysis of fMRI data using linear systems methods. NeuroImage, 6, 93-103.

Conlisk, J. \& Watts, H. (1979). A model for optimizing experimental designs for estimating response surfaces. Journal of Econometrics, 11, 27-42.

Crellin, N., Hastie, T., \& Johnstone, I. (1999). Statistical models for image sequences. Technical report, Department of Statistics, Stanford University, http://statistics.stanford.edu/ ckirby/techreports/BIO/BIO\%20205.pdf.

Culham, J. C. (2006). Functional neuroimaging: Experimental design and analysis. In R. Cabeza \& A. Kingstone (Eds.), Handbook of functional neuroimaging of cognition (2nd ed., pp. 53-82). Cambridge, MA: MIT Press.

Dale, A. M. (1999). Optimal experimental design for event-related fMRI. Human Brain Mapping, 8, 109-114.

Dale, A. M. \& Buckner, R. L. (1997). Selective averaging of rapidly presented individual trials using fMRI. Human Brain Mapping, 5, 329-340.

de Zwaart, J. A., Silva, A. C., van Gelderen, P., Kellman, P., Fukunaga, M., Chu, R., Koretsky, A. P., Frank, J. A., \& Duyn, J. H. (2005). Temporal dynamics of the BOLD fMRI impulse response. NeuroImage, 24, 667-677.

Desmond, J. E. \& Glover, G. H. (2002). Estimating sample size in functional MRI (fMRI) neuroimaging studies: Statistical power analysis. Journal of Neuroscience Methods, 118, $115-128$.

Dette, H., Martinez Lopez, I., Ortiz Rodriguez, I. M., \& Pepelyshev, A. (2006). Maximin efficient design of experiment for exponential regression models. Journal of Statistical Planning and Inference, 136, 4397-4418.

Di Salle, F., Formisano, E., Linden, D. E. J., Goebel, R., Bonavita, S., Pepino, A., Smaltino, F., \& Tedeschi, G. (1999). Exploring brain function with magnetic resonance imaging. European Journal of Radiology, 30, 84-94.

Diggle, P. J., Liang, K.-Y., \& Zeger, S. L. (1994). Anaysis of longitudinal data. Oxford: Clarendon Press. 
Donaldson, D. I. \& Buckner, R. L. (2001). Effective paradigm design. In P. Jezzard, P. M. Matthews, \& S. M. Smith (Eds.), Functional MRI: An introduction to methods (pp. 177-196). Oxford: Oxford University Press.

Flaherty, P., Jordan, M. I., \& Arkin, A. P. (2006). Robust design of biological experiments. In Y. Weiss, B. Schölkopf, \& J. Platt (Eds.), Advances in neural information processing systems 18 (pp. 363-370). Cambridge, MA: MIT Press.

Formisano, E., Esposito, F., Di Salle, F., \& Goebel, R. (2004). Cortex-based independent component analysis of fMRI time series. Magnetic Resonance Imaging, 22, 1493-1504.

Friston, K. J., Fletcher, P., Josephs, O., Holmes, A. P., Rugg, M. D., \& Turner, R. (1998). Event-related fMRI: Characterizing differential responses. NeuroImage, 7, 30-40.

Friston, K. J., Glaser, D. E., Henson, R. N. A., Kiebel, S., Philips, C., \& Ashburner, J. T. (2002). Classical and Bayesian inference in neuroimaging: Applications. NeuroImage, $16,484-512$.

Friston, K. J., Holmes, A. P., Poline, J.-B., Grasby, P. J., Williams, S. C. R., Frackowiak, R. S. J., \& Turner, R. (1995). Analysis of fMRI time-series revisited. NeuroImage, 2, $45-53$.

Friston, K. J., Jezzard, P., \& Turner, R. (1994). Analysis of functional MRI time-series. Human Brain Mapping, 1, 153-171.

Friston, K. J., Zarahn, E., Josephs, O., Henson, R. N. A., \& Dale, A. M. (1999). Stochastic designs in event-related fMRI. NeuroImage, 10, 607-619.

Gautama, T. \& Van Hulle, M. M. (2005). Estimating the global order of the fMRI noise model. NeuroImage, 26, 1211-1217.

Genovese, C. R., Lazar, N., \& Nichols, T. E. (2002). Thresholding of statistical maps in functional neuroimaging using the false discovery rate. NeuroImage, 15, 870-878.

Glover, G. H. (1999). Deconvolution of impulse response in event-related BOLD fMRI. NeuroImage, 9, 416-429.

Goebel, R., Muckli, L., Zanella, F. E., Singer, W., \& Stoerig, P. (2001). Sustained extrastriate cortical activation without visual awareness revealed by fMRI studies of hemianopic patients. Vision Research, 41, 1459-1474.

Gössl, C., Fahrmeir, L., \& Auer, D. P. (2001). Bayesian modeling of the hemodynamic response function in BOLD fMRI. NeuroImage, 14, 140-148.

Goutte, C., Nielsen, F. A., \& Hansen, L. K. (2000). Modeling the haemodynamic response in fMRI using smooth FIR filters. IEEE Transactions on Medical Imaging, 19(12), $1188-1201$. 
Hagberg, G. E., Zito, G., Patria, F., \& Sanes, J. N. (2001). Improved detection of eventrelated functional MRI signals using probability functions. NeuroImage, 14, 1193-1205.

Handwerker, D. A., Ollinger, J. M., \& D'Esposito, M. (2004). Variation of BOLD hemodynamic responses across subjects and brain regions and their effects on statistical analyses. NeuroImage, 21, 1639-1657.

Harville, D. A. (1997). Matrix algebra from a statistician's perspective. New York: Springer.

Harville, D. A. (2008). Matrix algebra from a statistician's perspective (2nd ed.). New York: Springer.

Hayasaka, S., Peiffer, A. M., Hugenschmidt, C. E., \& Laurienti, P. J. (2007). Power and sample size calculation for neuroimaging studies by non-central random field theory. NeuroImage, 37, 721-730.

Heckman, G. M., Bouvier, S. E., Carr, V. A., Harley, E. M., Cardinal, K. S., \& Engel, S. A. (2007). Nonlinearities in rapid event-related fMRI explained by stimulus scaling. NeuroImage, 34, 651-660.

Henson, R. N. A. (2004). Analysis of fMRI time series. In R. S. J. Frackowiak, K. J. Friston, C. D. Frith, R. J. Dolan, C. J. Price, S. Zeki, J. T. Ashburner, \& W. D. Penny (Eds.), Human brain function (2nd ed., pp. 793-822). Amsterdam: Elsevier.

Henson, R. N. A. (2007). Efficient experimental design for fMRI. In K. J. Friston, J. T. Ashburner, S. J. Kiebel, T. E. Nichols, \& W. D. Penny (Eds.), Statistical parametric mapping: The analysis of functional brain images (pp. 193-210). London: Academic Press.

Henson, R. N. A. \& Friston, K. J. (2007). Convolution models for fMRI. In K. J. Friston, J. T. Ashburner, S. J. Kiebel, T. E. Nichols, \& W. D. Penny (Eds.), Statistical parametric mapping: The analysis of functional brain images (pp. 178-192). London: Academic Press.

Honey, G. D. \& Bullmore, E. T. (2002). Functional neuroimaging and schizophrenia. Psychiatry, 1, 26-29.

Huettel, S. A. \& McCarthy, G. (2000). Evidence for a refractory period in the hemodynamic response to visual stimuli as measured by MRI. NeuroImage, 11, 547-553.

Huettel, S. A., Singerman, J. D., \& McCarthy, G. (2001). The effects of aging upon the hemodynamic response measured by functional MRI. NeuroImage, 13, 161-175.

Huettel, S. A., Song, A. W., \& McCarthy, G. (2004). Functional magnetic resonance imaging. Sunderland, MA: Sinauer Associates.

Josephs, O. \& Henson, R. N. A. (1999). Event-related functional magnetic resonance imaging: Modelling, inference and optimization. Philosophical Transcations of the Royal Society B: Biological Sciences, 354, 1215-1228. 
Josephs, O., Turner, R., \& Friston, K. J. (1997). Event-related fMRI. Human Brain Mapping, 5, 243-248.

Kanwisher, N., McDermott, J., \& Chun, M. M. (1997). The fusiform face area: A module in human extrastriate cortex specialized for face perception. The Journal of Neuroscience, $17(11), 4302-4311$.

Kao, M.-H., Mandal, A., Lazar, N., \& Stufken, J. (2007). Multi-objective experimental designs for event-related fMRI studies. Technical report, Department of Statistics, University of Georgia, http://www.stat.uga.edu/ amandal/fMRI.pdf.

Kao, M.-H., Mandal, A., Lazar, N., \& Stufken, J. (2009). Multi-objective optimal experimental designs for event-related fMRI studies. NeuroImage, 44, 849-856.

Kao, M.-H., Mandal, A., \& Stufken, J. (2009). Efficient designs for event-related functional magnetic resonance imaging with multiple scanning sessions. Communications in Statistics - Theory and Methods, 38(16), 3170-3182.

Kwong, K. K., Belliveau, J. W., Chesler, D. A., Goldberg, I. E., Weiskoff, R. M., Ponecelet, B. P., Kennedy, D. N., Hoppel, B. E., Cohen, M. S., Turner, R., Cheng, H.-M., Brady, T. J., \& Rosen, B. R. (1992). Dynamic magnetic resonance imaging of human brain activity during primary sensory stimulation. Proceedings of the National Academy of Sciences, USA, 89, 5675-5679.

Le, T. H., Patel, S., \& Roberts, T. P. L. (2001). Functional MRI of human auditory cortex using block and event-related designs. Magnetic Resonance in Medicine, 45, 254-260.

Lindquist, M. A. (2008). The statistical analysis of fMRI data. Statistical Science, 23(4), 439-464.

Lindquist, M. A., Loh, J. M., Atlas, L. Y., \& Wager, T. D. (2009). Modeling the hemodynamic response function in fMRI: Efficiency, bias and mis-modeling. NeuroImage, 45, S187-S198.

Lindquist, M. A. \& Wager, T. D. (2007). Validity and power in hemodynamic response modeling: A comparison study and a new approach. Human Brain Mapping, 28, 764784 .

Liu, T. T. (2004). Efficiency, power and entropy in event-related fMRI with multiple trial types, Part II: Design of experiments. NeuroImage, 21, 401-413.

Liu, T. T. \& Frank, L. R. (2004). Efficiency, power, and entropy in event-related fMRI with multiple trial types, Part I: Theory. NeuroImage, 21, 387-400.

Liu, T. T., Frank, L. R., Wong, E. C., \& Buxton, R. B. (2001). Detection power, estimation efficiency, and predictability in event-related fMRI. NeuroImage, 13, 759-773.

Logothetis, N. K. \& Wandell, B. A. (2004). Interpreting the BOLD signal. Annual Review of Physiology, 66, 735-769. 
Lovell, M. C. (1963). Seasonal adjustments of economic time series and multiple regression. Journal of the American Statistical Association, 58(304), 993-1010.

Maus, B., van Breukelen, G. J. P., Goebel, R., \& Berger, M. P. F. (2010a). Optimization of blocked designs in fMRI studies. Psychometrika, 75 (2), 373-390.

Maus, B., van Breukelen, G. J. P., Goebel, R., \& Berger, M. P. F. (2010b). Robustness of optimal design of fMRI experiments with application of a genetic algorithm. NeuroImage, 49, 2433-2443.

Miezin, F. M., Maccotta, L., Ollinger, J. M., Petersen, S. E., \& Buckner, R. L. (2000). Characterizing the hemodynamic response: Effects of presentation rate, sampling procedure, and the possibility of ordering brain activity based on relative timing. NeuroImage, $11,735-759$.

Moerbeek, M., van Breukelen, G. J. P., \& Berger, M. P. F. (2008). Optimal designs for multilevel studies. In J. de Leeuw \& E. Meijer (Eds.), Handbook of multilevel analysis (pp. 177-205). New York: Springer.

Mohamed, F. B., Tracy, J. I., Faro, S. H., Emperado, J., Koenigsberg, R., Pinus, A., \& Tsai, F. Y. (2000). Investigation of alternating and continuous experimental task designs during single finger opposition fMRI: A comparative study. Journal of Computer Assisted Tomography, 24 (6), 935-941.

Mumford, J. A. \& Nichols, T. E. (2008). Power calculation for group fMRI studies accounting for arbitrary design and temporal autocorrelation. NeuroImage, 39, 261-268.

Murphy, K. \& Garavan, H. (2004). An empirical investigation into the number of subjects required for an event-related fMRI study. NeuroImage, 22, 879-885.

Nakai, T., Matsumura, A., Nose, T., Kato, C., Glover, G. H., \& Matsuo, K. (2003). The effect of task block arrangement on the detectability of activation in fMRI. Magnetic Resonance Imaging, 21, 941-947.

Ogawa, S., Lee, T.-M., Nayak, A. S., \& Glynn, P. (1990). Oxygenation-sensitive contrast in magnetic resonance image of rodent brain at high magnetic fields. Magnetic Resonance in Medicine, 14(1), 68-78.

Ollinger, J. M., Corbetta, M., \& Shulman, G. L. (2001). Separating processes within a trial in event-related functional MRI, II. Analysis. NeuroImage, 13, 218-229.

Ollinger, J. M., Shulman, G. L., \& Corbetta, M. (2001). Separating processes within a trial in event-related functional MRI: I. The method. NeuroImage, 13, 210-217.

Ouwens, M. J. N. M., Tan, F. E. S., \& Berger, M. P. F. (2002). Maximin D-optimal designs for longitudinal mixed effects models. Biometrics, 58, 735-741. 
Owen, A. M., Epstein, R., \& Johnsrude, I. S. (2001). FMRI: Applications to cognitive neuroscience. In P. Jezzard, P. M. Matthews, \& S. M. Smith (Eds.), Functional MRI: An introduction to methods (pp. 311-327). Oxford: Oxford University Press.

Penny, W. D. \& Holmes, A. P. (2004). Random-effects analysis. In R. S. J. Frackowiak, K. J. Friston, C. D. Frith, R. J. Dolan, C. J. Price, S. Zeki, J. T. Ashburner, \& W. D. Penny (Eds.), Human brain function (2nd ed., pp. 843-850). Amsterdam: Elsevier.

Penny, W. D., Stephan, K. E., Mechelli, A., \& Friston, K. J. (2004). Modelling functional integration: A comparison of structural equation and dynamic causal models. NeuroImage, 23, S264-S274.

Pollmann, S., Wiggins, C. J., Norris, D. G., von Cramon, D. Y., \& Schubert, T. (1998). Use of short intertrial intervals in single-trial experiments: A $3 \mathrm{~T}$ fMRI-study. NeuroImage, 8, 327-339.

Pronzato, L. \& Walter, E. (1988). Robust experiment design via maximin optimization. Mathematical Biosciences, 89, 161-176.

Purdon, P. L. \& Weisskoff, R. M. (1998). Effect of temporal autocorrelation due to physiological noise and stimulus paradigm on voxel-level false-positive rates in fMRI. Human Brain Mapping, 6, 239-249.

Rajapakse, J. C., Kruggel, F., Maisog, J. M., \& von Cramon, D. Y. (1998). Modeling hemodynamic response for analysis of functional MRI time-series. Human Brain Mapping, 6, $283-300$.

Rosen, B. R., Buckner, R. L., \& Dale, A. M. (1998). Event-related functional MRI: Past, present and future. Proceedings of the National Academy of Sciences, USA, 95, 773-780.

Schouten, H. J. A. (1999). Sample size formula with a continuous outcome for unequal group sizes and unequal variances. Statistics in Medicine, 18, 87-97.

Seber, G. A. F. (1977). Linear regression analysis. New York: Wiley.

Silvey, S. D. (1980). Optimal design. London: Chapman and Hall.

Sim, R. \& Roy, N. (2005). Global A-optimal robot exploration in SLAM. In Proceedings of the IEEE/RSJ international conference of robotics and automation (ICRA 2005), (pp. 661-666).

Skudlarski, P., Constable, R. T., \& Gore, J. C. (1999). ROC analysis of statistical methods used in functional MRI: Individual subjects. NeuroImage, 9, 311-329.

Smith, S. M., Jenkinson, M., Beckmann, C. F., Miller, K., \& Woolrich, M. W. (2007). Meaningful design and contrast estimability in FMRI. NeuroImage, 34, 127-136. 
Suckling, J., Ohlssen, D., Andrew, C., Johnson, G., Williams, S. C. R., Graves, M., Chen, C.-H., Spiegelhalter, D., \& Bullmore, E. (2008). Components of variance in a multicentre functional MRI study and implications for calculation of statistical power. Human Brain Mapping, 29, 1111-1122.

Wager, T. D. \& Nichols, T. E. (2003). Optimization of experimental design in fMRI: A general framework using a genetic algorithm. NeuroImage, 18, 293-309.

Wager, T. D., Vazquez, A., Hernandez, L., \& Noll, D. C. (2005). Accounting for nonlinear BOLD effects in fMRI: Parameter estimates and a model for prediction in rapid eventrelated studies. NeuroImage, 25, 206-218.

Woolrich, M. W., Behrens, T. E. J., \& Smith, S. M. (2004). Constrained linear basis sets for HRF modelling using variational Bayes. NeuroImage, 21, 1748-1761.

Woolrich, M. W., Ripley, B. D., Brady, M., \& Smith, S. M. (2001). Temporal autocorrelation in univariate linear modeling of FMRI data. NeuroImage, 14, 1370-1386.

Worsley, K. J. (2005). Spatial smoothing of autocorrelations to control the degrees of freedom in fMRI analysis. NeuroImage, 26, 635-641.

Worsley, K. J. \& Friston, K. J. (1995). Analysis of fMRI time-series revisited-again. NeuroImage, 2, 173-181.

Worsley, K. J., Liao, C. H., Aston, J., Petre, V., Duncan, G. H., Morales, F., \& Evans, A. C. (2002). A general statistical analysis for fMRI data. NeuroImage, 15, 1-15.

Worsley, K. J., Marrett, S., Neelin, P., Vandal, A. C., Friston, K. J., \& Evans, A. C. (1996). A unified statistical approach for determining significant signals in images of cerebral activation. Human Brain Mapping, 4, 58-73.

Wüstenberg, T., Giesel, F. L., \& Strasburger, H. (2005). Methodische Grundlagen der Optimierung funktioneller MR-Experimente. Radiologe, 45, 99-112.

Zarahn, E., Aguirre, G. K., \& D'Esposito, M. (1997). A trial based experimental design for fMRI. NeuroImage, 6, 122-138.

Zarahn, E. \& Friston, K. J. (2002). Some limit results for efficiency in stochastic fMRI designs. Biometrical Journal, 44, 496-509. 


\section{Acknowledgments}

I am grateful to many people for their help, both direct and indirect, in writing this thesis which would have never been finished without the support and good will of my colleagues, friends and family.

First of all, I would like to express my gratitude to my supervisors for their continued support and valuable guidance: Prof. Martijn Berger, prof. Rainer Goebel and dr. Gerard van Breukelen. Martijn, thank you for keeping me focused and on track day by day. Your comments on my articles during our meetings and discussions also helped my articles to be more focused and improved my writing skills. Rainer, thank you for sharing your deep knowledge about neuroimaging and fMRI with Gerard, Martijn and me. I really appreciate that you explained the mysteries of fMRI to us and helped us to find the relevant issues to tackle. Gerard, thank you for your critical, thoughtful and detailed comments on my research, your kind encouragement, your confidence in me and your reliable guidance. You would always find time for me in your busy agenda which I deeply appreciate. I have learned and benefited much from my work with you and our fruitful discussions.

I would like to thank the members of the assessment committee: Prof. Leopold Curfs, dr. Elia Formisano, prof. David Norris, prof. John Stufken and dr. Frans Tan. Frans, thank you also for all the insightful discussions about optimal design. Furthermore, I am grateful to the corona of my $\mathrm{PhD}$ defense. I want to express my gratitude to the supervisors of my master thesis, prof. Holger Dette and prof. Ansgar Steland, for their support during my master period. Dr. Stefan Boehringer, thank you for the opportunity to do research while I was still a student.

To my former and current room- and fate-mates Fetene Bekele Tekle, Shirley Ortega Azurduy, Stéphanie van Nispen and Haftom Temesgen: Thank you for our discussions, your advices, your positive support and help. Stéphanie, you were such a nice roommate and you are such a good friend. Thank you for this! Shirley, I appreciate all the help with LaTeX and your quick answers to my mails even after leaving the department. Fetene and Haftom, thank you for being so relaxed and calm roommates. I am sure that this also had a good influence on me!

My current and former colleagues at the Department of Methodology and Statistics, 
Maastricht University, have been helpful and kind to me. Many thanks to you all for the help, technical and administrative assistance, social moments and fruitful discussions. In particular, I want to thank Marga Doyle for the administrative assistance and corrections of my Dutch summary, Hans Hilberink for the technical assistance, and Wolfgang Viechtbauer as well as Jan Serroyen for the technical assistance with the simulation computer. Jimmie Leppink also helped me with my Dutch summary. Jimmie, thank you for the corrections which really improved the summary! I am grateful for the opportunity to continue as a post-doc at the department and I want to thank everyone who was involved in this decision. Further, I would like to thank Fabrizio Espositio for providing me with fMRI data and all his explanations to my questions. Many thanks also go to the members of the Journal Club Maastricht (JCM) for the interesting discussions about various articles, the tasty food, the pleasant atmosphere during our dinners and during all other activities.

I am fortunate to have good friends and to have found new friends in Maastricht which all contributed to my general well-being. Thanks to all of you in my hometown Hückelhoven, in my university town Bochum, in Maastricht and elsewhere in the world! Ich bedanke mich besonders bei meinen Eltern Mechthilde und Friedrich, meiner Schwester Birgit und meinem Schwager Jens, meinem Bruder Stefan und meiner Schwägerin Katja für die logistische und mentale Unterstützung. Ohne euch, im Besonderen meine Eltern, wäre dies alles nicht möglich gewesen! Now, I have to switch back to English for my boyfriend Chris. Thank you for your support, love, understanding, sense of humor, patience and your nice corrections on my English writing! You made the end of this $\mathrm{PhD}$ journey more joyful than I would have ever imagined to be possible. 


\section{About the author}

Bärbel Maus was born in Linnich, Germany, on February 7, 1981. She attended secondary school at the Gymnasium Hückelhoven from 1991 to 2000. Subsequently, she started studying mathematics with biology as minor subject at the Ruhr-University Bochum, Germany. In the academic year 2002/2003 she studied at the Université Rouen, France, with an Erasmus scholarship. During her further studies in Bochum she specialized in statistics and worked as a student assistant at the Institute for Human Genetics, University DuisburgEssen, Germany, where she carried out research and statistical analyses for computerassisted classification of patients based on digital images. She finished her studies successfully in 2006 with her diploma thesis on statistical genetics and obtained the degree Diplom-Mathematikerin (equivalent to master). In October 2006 she started as a PhD candidate at the Department of Methodology and Statistics, Maastricht University. Her research focused on optimal design problems for functional magnetic resonance imaging (fMRI) and led to this dissertation. Since January 2011 she has been working at the same department as a postdoc to continue her research on optimal design for fMRI experiments and statistical analysis of fMRI data. 